\title{
Limit groups over partially commutative groups and group actions on real cubings
}

\author{
MontserRat CASAls-Ruiz \\ ILYA KAZACHKOV
}

The study of limit groups, that is, finitely generated fully residually free groups, was a key first step towards the understanding of the elementary theory of a free group. In this paper we conduct a systematic study of the class $\mathfrak{U}$ of finitely generated fully residually partially commutative groups.

Our first main goal is to give an algebraic characterisation of the class $\mathfrak{U}$ : a finitely generated group $G$ is fully residually partially commutative if and only if it is a subgroup of a graph tower (a group built hierarchically using partially commutative groups and (nonexceptional) surfaces.) Furthermore, if the group $G$ is given by its finite radical presentation, then the graph tower and the embedding can be effectively constructed. This result generalises the work of Kharlampovich and Miasnikov on fully residually free groups.

Following Sela's approach to limit groups, the second goal of the paper is to provide a dynamical characterisation of the class $\mathfrak{U}$. We introduce a class of spaces, called real cubings, as higher-dimensional generalisations of real trees and show that a specific type of action on these spaces characterises the class $\mathfrak{U}$ : a finitely generated group acts freely cospecially on a real cubing if and only if it is fully residually partially commutative. As a corollary we get that (geometric) limit groups over partially commutative groups are fully residually partially commutative. This result generalises the work of Sela on limit groups over free groups.

20F65, 20F67; 20F70, 20E08

\section{Introduction}

Around 1945, A Tarski asked whether or not free groups have the same elementary theory and if their theory is decidable. Since then, Tarski problems have motivated a large body of research which has uncovered deep connections between model theory, geometry and group theory and served as a nexus for many now classical results in geometric group theory and theoretical computer science. Tarski problems were finally solved in 2006 in a series of papers; see Sela [54] and Kharlampovich and Myasnikov [41] and references there. 
A key step in the work on Tarski problems was the study of groups that belong to the universal class of free groups. In his work [49], Remeslennikov established a link between model theory and group theory by showing that the class of groups universally equivalent to a free group is exactly the class of fully residually free groups, that is, the class of groups for which, given any finite set of elements from a fixed group, there exists a homomorphism from the group to a free group which is injective on this set. In [3], Baumslag, Myasnikov and Remeslennikov characterised this class of groups in algebro-geometric terms and show that finitely generated fully residually free groups are coordinate groups of irreducible algebraic sets over free groups.

First structural results on fully residually free groups were obtained by Kharlampovich and Miasnikov in [38]. In their work, the authors show that limit groups can be obtained recursively from free groups, (nonexceptional) surface groups and free abelian groups by a finite sequence of amalgamated free products and HHN-extensions over $\mathbb{Z}$. Among other things, the existence of such decomposition implies that finitely generated fully residually free groups are finitely presented and, moreover, coherent.

In his work, Sela suggested a new, more geometric approach to the analysis of Diophantine sets and the study of first order properties of free groups; see [52]. Sela characterised the multifaceted class of fully residually free groups via their actions on real trees and popularised these groups as limit groups. Using structural theorems for groups acting on real trees, the author obtained a hierarchical description of limit groups in terms of their JSJ-decomposition. Following Sela's approach, Champetier and Guirardel gave a topological characterisation of the class of limit groups as limits of free groups in a compact space of marked groups and proved that this class is the smallest containing finitely generated free groups, and stable under free products and under taking generalised doubles over a group in the class; see Champetier and Guirardel [15]. The versatile nature and rich structure of the class of limit groups made them an object of intense study.

Inside the class of limit groups there is a prominent subclass that clearly stands out: the class of $\omega$-residually free towers (alias NTQ-groups). A finitely generated group is an $\omega$-residually free tower if it belongs to the smallest class of groups containing finitely generated free groups and (nonexceptional) surface groups, which is stable under taking free products, free extensions of centralisers, and attaching retracting surfaces along maximal cyclic subgroups.

The importance of this class is two-fold. On the one hand, $\omega$-residually free towers can be used to characterise limit groups, namely a group is a limit group if and only if it is a subgroup of an $\omega$-residually free tower. This characterisation is crucial in the work of Kharlampovich and Miasnikov to determine the algebraic structure and finiteness 
properties of limit groups (see also [15]). On the other hand, towers are key to describe the class of finitely generated groups elementarily equivalent to a free group:

Theorem (Kharlampovich and Miasnikov [41]; Sela [54]) A finitely generated group $G$ is elementarily equivalent to a free group if and only if it is a hyperbolic $\omega$-residually free tower.

The main goal of this paper is to undertake a systematic study of the universal class defined by a partially commutative group. Recall that a group $\mathbb{G}$ is called partially commutative (or right-angled Artin) if it is given by a presentation

$$
G=\left\langle x_{1}, \ldots, x_{n} \mid R\right\rangle
$$

where $R \subset\left\{\left[x_{i}, x_{j}\right]=1 \mid i, j=1, \ldots, n, i \neq j\right\}$.

Some of the aforementioned characterisations of limit groups are of general nature and can be restated in the setting of partially commutative groups; see Baumslag, Myasnikov and Remeslennikov [3], Myasnikov and Remeslennikov [47] and Daniyarova, Myasnikov and Remeslennikov [19] (recall that partially commutative groups are linear and hence equationally Noetherian).

Theorem [19] Let $\mathbb{G}$ be a partially commutative group and $G$ a finitely generated group. Then the following statements are equivalent:

- $G$ is universally equivalent to $\mathbb{G}$.

- $\quad G$ is fully residually $\mathbb{G}$.

- $G$ is the coordinate group of an irreducible algebraic set over $\mathbb{G}$.

Our first main goal is to describe the algebraic structure of finitely generated fully residually partially commutative groups. A naturally arising obstacle here is that the structure of these groups is at least as complex as the structure of subgroups of partially commutative groups. In turn, subgroups of partially commutative groups are known to be very diverse: surface groups and graph braid groups as well as fundamental groups of CAT(0) 3-manifolds are virtually subgroups of partially commutative groups (see Crisp and Wiest [18] and Liu [43]); and complicated: some of them exhibit remarkable finiteness properties (see Stallings [56], Bieri [6] and Bestvina and Brady [5]). With this in mind, our strategy is to introduce a new class of groups with a solid algebraic structure that we call graph towers, and prove that any finitely generated fully residually partially commutative group is a subgroup of a graph tower. 
Graph towers, as generalisations of $\omega$-residually free towers, can be loosely described as the smallest class of groups containing partially commutative groups and (nonexceptional) surface groups, which is stable under taking graph products, (free) extensions of centralisers, and attaching retracting surfaces along maximal cyclic subgroups (the way the surfaces are attached is using some type of "graph product with amalgamation"; see definition below). This endows graph towers with two natural underlying structures (which naturally hints at their name): the tower structure, as quotients of $\omega$-residually free towers by (the normal subgroup generated by a set of) commutation relations; and the graph product structure, as quotients of some partially commutative group by a specific set of relations (surface and commutation relations). More formally, graph towers are defined recursively using algebraic constructions as follows. Graph towers of height 0 are partially commutative groups. A graph tower $\mathfrak{T}^{l}$ of height $l$ is constructed by taking an amalgamated product (and HNN-extension) of a graph tower $\mathfrak{T}^{l-1}$ of height $l-1$ and a direct product of either a free abelian group, or a free group or a surface group with boundary, and the centraliser $C_{\mathfrak{T}^{l-1}}(D)$ of (a certain) subgroup $D$ of $\mathfrak{T}^{l-1}$, where the amalgamation is taken over this centraliser $C_{\mathfrak{T}^{l-1}}(D)$. More precisely, we have:

Definition (see Lemma 5.3) Let partially commutative groups be graph towers of height 0 . Assume that graph towers $\mathfrak{T}^{l-1}$ of height $l-1$ have been constructed. Then a graph tower of height $l$ has one of the following presentations:

(a1) $\mathfrak{T}^{l-1} * C_{\mathfrak{T}^{l-1}(D)}\left(C_{\mathfrak{T}^{l-1}}(D) \times\left\langle x_{1}^{l}, \ldots, x_{m_{l}}^{l}\right\rangle\right)$ (basic type, $D$ is a certain nonabelian subgroup of $\left.\mathfrak{T}^{l-1}\right)$.

(a2) $\mathfrak{T}^{l-1} *_{\mathfrak{T}^{l-1}(D)}\left(C_{\mathfrak{T}^{l-1}}(D) \times\left\langle x_{1}^{l}, \ldots, x_{m_{l}}^{l} \mid\left[x_{i}^{l}, x_{j}^{l}\right]=1,1 \leq i, j \leq m_{l}, i \neq j\right\rangle\right)$ (basic type, $D$ is a certain abelian subgroup of $\mathfrak{T}^{l-1}$ ).

(b1) $\mathfrak{T}^{l-1} * C_{\mathfrak{T}^{l-1}(u)}\left(C_{\mathfrak{T}^{l-1}}(u) \times\left\langle x_{1}^{l}, \ldots, x_{m_{l}}^{l} \mid\left[x_{i}^{l}, x_{j}^{l}\right]=1,1 \leq i, j \leq m_{l}, i \neq j\right\rangle\right)$ (abelian type, $u$ is a nontrivial irreducible root element; see page 735 for the definition).

(b2) $\mathfrak{T}^{l-1} *_{\mathfrak{T}^{l-1}(D)}\left(C_{\mathfrak{T}^{l-1}}(D) \times\left\langle x_{1}^{l}, \ldots, x_{m_{l}}^{l} \mid\left[x_{i}^{l}, x_{j}^{l}\right]=1,1 \leq i, j \leq m_{l}, i \neq j\right\rangle\right)$ (abelian type, $D$ is a certain nonabelian subgroup of $\mathfrak{T}^{l-1}$ ).

(c) $\mathfrak{T}^{l-1} *_{C_{\mathfrak{T} l-1}(D) \times\left\langle u_{2 g+1}, \ldots, u_{m}\right\rangle}\left(\left\langle u_{2 g+1}, \ldots, u_{m}, x_{1}^{l}, \ldots, x_{m_{l}}^{l} \mid W\right\rangle \times C_{\mathfrak{T}^{l-1}}(D)\right)$ (surface type, $W$ is a nonexceptional quadratic equation and $D$ is a certain nonabelian subgroup of $\mathfrak{T}^{l-1}$ ).

At this point, we are vague about the conditions on the subgroup $D$. This makes some cases to seem redundant: (b1) seems a particular case of (a2). The definition will be made precise and the difference will be clear when constructing the graph towers; see 
Section 5. Informally, the subgroups $D$ of $\mathfrak{T}^{l-1}$ behave as directly indecomposable canonical parabolic subgroups (see page 735 for definition), ie they are subgroups generated by some generators of $\mathfrak{T}^{l-1}$ which are discriminated (in a "minimal way") into directly indecomposable canonical parabolic subgroups of the partially commutative group $\mathbb{G}$; hence, since $u$ need not be a generator, formally it does not belong to the subgroup $D$; see Section 5 .

Notice that the class of graph towers naturally extends the class of $\omega$-residually free towers. Indeed, if the subgroup $D$ of an $\omega$-residually free tower is nonabelian, then since centralisers in a freely discriminated group $\mathfrak{T}^{l-1}$ are commutative transitive, it follows that $C_{\mathfrak{T}^{l-1}}(D)$ is trivial and so, in this case, the decompositions listed in the definition correspond to free products, and amalgamated products and HNN-extensions (over infinite cyclic groups). If the subgroup $D$ is abelian, then $C_{\mathfrak{T}^{l-1}}(D)$ is a maximal abelian subgroup of $\mathfrak{T}^{l-1}$ and the decomposition corresponds to an amalgamated product over a (finitely generated) free abelian group.

Once the class of graph towers is introduced, we are in the position to state one of the main results of our paper.

Theorem A finitely generated group $G$ is a fully residually partially commutative group if and only if it is a subgroup of a graph tower.

Furthermore, if the groups $G$ is given by its finite radical presentation, then the corresponding graph tower $\mathfrak{T}$ and the embedding of $G$ into $\mathfrak{T}$ can be constructed effectively.

This result generalises the work in [38] (see also [15]) which is foundational in the solution of Tarski problems for free groups.

As we already mentioned above, partially commutative groups are equationally Noetherian and hence any finitely generated residually $\mathbb{G}$ group is a subdirect product of the direct product of finitely many fully residually $\mathbb{G}$ groups; see the authors [13] and [3]. We show that such an embedding can be given effectively.

Theorem Let $G$ be a finitely generated residually $\mathbb{G}$ group given by its finite radical presentation. Then one can effectively construct an embedding of $G$ into the direct product of finitely many fully residually $\mathbb{G}$ groups.

An analogous result for free groups was proven by Kharlampovich and Miasnikov in [38] and later by Bridson, Howie, Miller and Short in their work on the structure of finitely presented residually free groups; see [10]. 
All of the aforementioned results are based on the combinatorial process to describe homomorphisms from any finitely generated group to partially commutative groups developed by the authors in [14].

In more geometric terms, Sela characterised limit groups via their action on real trees. He noticed that groups that admit infinitely many (nonconjugate) homomorphisms to a free group have a special type of action on real trees and defined limit groups as quotients of these groups by the (stable) kernel of the action. He then used structural theorems on groups acting on real trees to describe the hierarchical structure of limit groups in terms of their abelian JSJ-decomposition. When one tries to pursue an analogous geometric approach for limit groups over partially commutative groups, one immediately runs into some natural difficulties, namely:

- On what type of spaces do limit groups over partially commutative groups act? What is the geometry of these spaces?

- Are there any structural theorems for groups acting on these spaces (eg an analogue of Rips' theorem for groups acting on real trees)?

- What type of decomposition describes the structure of limit groups over partially commutative groups?

In Section 9 we conduct the first steps to address the aforementioned questions: we introduce a new class of spaces, that we call real cubings on which limit groups over partially commutative groups act naturally and prove structural results for a specific type of group actions on real cubings which we call essentially free cospecial. The type of action we study is restrictive and in fact we show that it characterises the class of limit groups over partially commutative groups. Namely, we prove:

Theorem Let $G$ be a finitely generated group. The group $G$ acts essentially freely cospecially on a real cubing if and only if it is a limit group over a partially commutative group if and only if it is a subgroup of a graph tower.

As a corollary, we obtain a generalisation of Sela's characterisation of (geometric) limit groups as fully residually free groups.

Corollary A finitely generated group $G$ is a (geometric) limit group over a partially commutative group $\mathbb{G}$ if and only if it is fully residually $\mathbb{G}$.

In the case of free actions, the above theorem results in the following corollary, which can be likened to Rips' theorem on free actions on real trees (see Section 10 for further discussion). 
Corollary A finitely generated group $G$ acts freely, essentially freely and cospecially on a real cubing if and only if $G$ is a subgroup of the graph product of free abelian and (nonexceptional) surface groups.

In particular, if the real cubing is a real tree, then $G$ is a (subgroup of) the free product of free abelian groups and (nonexceptional) surface groups.

The results of our work bring out some interesting questions and new directions as well as suggest ways to approach them. We now briefly list some of them and refer the reader to Section 10 for further discussion.

Firstly, the structural results on limit groups over partially commutative groups and graph towers open ways to tackle a number of questions for these classes of groups, for instance:

- Are the conjugacy and isomorphism problems decidable for graph towers?

- Are graph towers hereditary conjugacy separable?

- Are quasiconvex subgroups of graph tower over partially commutative groups virtual retracts?

- Are graph towers virtually special?

- What is the rank gradient and homology of a graph tower?

- Do partially commutative groups have finite Krull dimension?

In a different vein, our work points towards a generalisation of Rips' theory of groups acting on real trees. In the free group case, Rips' machine takes a (free) action of a finitely presented group on a real tree and analyses the dynamics of this action. The machine shows that the action admits a decomposition in simpler pieces and that these pieces correspond to dense actions of abelian groups on a line, or actions of a surface group on a tree dual to a foliation, or are simplicial or Levitt-type actions of a free group. When stated this way, there is no obvious connection between Rips' machine and the Makanin-Razborov process for analysing homomorphisms. However, both procedures are closely related. A key point is that using the geometry of real trees, one can encode the dynamics of the group's action into a foliated band complex; in turn, band complexes can be likened to generalised equations and the final punchline is that the process to analyse generalised equations (the Makanin-Razborov process) can be naturally extended to analyse foliated band complexes. Our hope is that good actions on real cubings can be encoded into multifoliated band complexes, which can be interpreted as constrained generalised equations and that the procedure we describe in this paper will guide the analysis of the corresponding dynamics. 
Last but not least, just as the class of $\omega$-residually free towers plays a crucial role in the classification of groups elementarily equivalent to a free group [41; 54], it is our hope that the class of graph towers will play a similar role in the classification of groups elementarily equivalent to a given partially commutative group.

\section{Organisation of the paper}

We now outline the organisation of the paper. In Section 2, we review basic notions and fix notation on partially commutative groups and algebraic geometry over groups.

Before we describe the content of the next sections, we present the strategy for our approach and explain the difficulties that one needs to overcome. In the free group case, Kharlampovich and Miasnikov showed that given a limit group $G$ one can effectively construct an $\omega$-residually free tower $\mathfrak{T}_{G}$ and an embedding $i: G \rightarrow \mathfrak{T}_{G}$. This construction essentially relies on the Makanin-Razborov process for describing the set of homomorphisms from $G$ to a free group. The Makanin-Razborov diagram is a finite rooted tree, whose vertices $v$ are labelled by finitely generated groups $G_{v}$ together with (a subgroup of) the automorphism group of $G_{v}$; leaves are just labelled by free groups; and edges $\left(v, v^{\prime}\right)$ are labelled by proper epimorphisms from $G_{v}$ to $G_{v^{\prime}}$. This diagram describes the set of all homomorphisms from $G$ to a free group, namely each homomorphism $\varphi$ from $G$ to a free group "factors" through a branch $v_{0}, v_{1}, \ldots, v_{k}$ of the diagram, ie $\varphi$ is the composition $\psi_{0} \pi_{v_{0}, v_{1}} \psi_{1} \cdots \pi_{v_{k-1}, v_{k}} \phi$, where $\psi_{i}$ is an automorphism of group associated to the vertex $v_{i}, \pi_{v_{i}, v_{i+1}}$ is the epimorphism labelling the edge $\left(v_{i}, v_{i+1}\right)$ and $\phi$ is a homomorphism from the free group associated to the leaf $v_{k}$ to a free group.

Each vertex $v$ in the diagram has an associated type: abelian, surface, linear or trivial. In turn, each of these types determines a splitting in the JSJ-decomposition of $G_{v}$ (or a free splitting), that is, a decomposition of $G_{v}$ as an amalgamated product (or HNN-extension) over an abelian (maybe trivial) subgroup. Furthermore, since the group $G$ is a limit group (that is fully residually free), one can show that there is a discriminating family that factors through a branch of the diagram. Using induction on the height of this branch and taking into consideration the types of the vertices in the branch and so the structure of the groups that occur in the branch, one constructs the $\omega$-residually free tower and the corresponding embedding.

Along these lines, we expected to use the analogue of the Makanin-Razborov process for partially commutative groups presented in [14] to construct the corresponding graph towers. However, there is an essential difference: the type of a vertex alone does not determine the structure of the corresponding group. The main obstruction to obtaining 
splittings is the presence of commutation relations in the presentation; see Example 3.1 in Section 3.

A key notion to overcome this setback is that of tribes. Roughly speaking, two generators belong to the same tribe if the set of generators with whom they commute is the same, ie the generators $x_{i}$ and $x_{j}$ of $G_{v}$ belong to the same tribe if for any $x_{k}$ the commutator $\left[x_{i}, x_{k}\right]$ is a relation of $G_{v}$ if and only if so is $\left[x_{j}, x_{k}\right]$.

If the type of the vertex $v$ is abelian, then, as in the free group case, we do obtain a splitting. If the type of a vertex is quadratic (type 12), then we show that if the group of automorphisms associated to the vertex is nontrivial and so there is dynamics associated to $G_{v}$, then this dynamics occurs inside a tribe. Thus, in the quadratic case, it is the presence of nontrivial dynamics that assures the splitting of the group. However, this approach is insufficient to obtain a splitting in the linear case: even when the group of automorphisms is nontrivial, we could not directly prove that then the dynamics occur inside a tribe and obtain an splitting of the group.

Intuitively, the main difference between the quadratic and linear cases is that the dynamics in the quadratic case, as in the free group case, is "mixing" and this forces uniformity on the type of commutation constraints involved in the action which in turn implies that the dynamics occur inside a tribe; however, in the linear case the dynamics need not be "mixing" and so we cannot use it to get control on the commutation constraints.

For this reason, we were forced to modify the treatment of the linear case in the process presented in [14]. The main idea is to force by construction that the dynamics in the linear case happen inside a tribe. This is achieved by adding a new case in the description of our process from [14, Case 6.5]. The main goal of Section 3.1 is to show that under this additional assumption on the linear case, the main result of [14] still holds, that is one can still construct a finite Makanin-Razborov-type diagram that describes all homomorphisms (and for which the dynamics of the groups associated to the vertices are inside a tribe). We call this new diagram, the tribal solution tree.

In Section 4, we show that the groups $G_{v}$ associated to vertices of the tribal solution tree that have nontrivial dynamics do split, that is they can be presented as amalgamated products (and HNN-extensions); we further find the explicit presentations for these groups.

In Section 5 we introduce the notion of graph tower and in Section 6 we show how to associate a graph tower to a given branch of a tribal solution tree.

In Section 7 we prove that the graph towers we constructed are discriminated by a family of homomorphisms induced by the homomorphisms that factor through the branch of the tribal solution tree that defines the tower. 
In Section 8 we prove our embedding results.

Theorem Given a limit group $G$ over a partially commutative group $\mathbb{G}$ one can effectively construct a graph tower $\mathfrak{T}$ and an embedding $i: G \rightarrow \mathfrak{T}$.

Given a residually $\mathbb{G}$ group $G$ one can effectively construct finitely many limit groups $G_{1}, \ldots, G_{k}$ and an embedding of $G$ into the direct product $G_{1} \times \cdots \times G_{k}$.

In Section 9, we take a more geometric perspective and define real cubings as ultralimits of cubings of bounded width. We then introduce a specific type of group actions on real cubings that we call essentially free cospecial. We proceed to show that these actions characterise the class of limit groups and give a generalisation of Sela's result on geometric limit groups over free groups and fully residually free groups by proving that (geometric) limit groups over a partially commutative group $\mathbb{G}$ are fully residually $\mathbb{G}$.

Finally, in the last section, we discuss some open problems and give an outlook.

Acknowledgements The authors thank Mark Sapir and Vincent Guirardel for stimulating discussions. We are extremely grateful to the referee for his/her careful and thoughtful reading and constructive remarks that helped us to improve the exposition of this paper.

The first author is supported by the Marie Curie International Incoming Fellowship within the $7^{\text {th }}$ European Community Framework Programme. The second author is supported by the Royal Commission 1851 Research Fellowship and the ERC Starting Grant number PCG-336983. Both authors are partly supported by the Spanish Government, grant number MTM2011-28229-C02-02, partly with FEDER funds.

\section{Basics}

\subsection{Partially commutative groups}

In this section we recall some preliminary results on partially commutative groups and introduce the notation we use throughout the text.

Let $\Gamma=(V(\Gamma), E(\Gamma))$ be a (undirected) simplicial graph. Then the partially commutative group $\mathbb{G}=\mathbb{G}(\Gamma)$ defined by the (commutation) graph $\Gamma$ is the group given by the presentation

$$
\left.\mathbb{G}=\langle V(\Gamma)|\left[v_{1}, v_{2}\right]=1 \text { whenever }\left(v_{1}, v_{2}\right) \in E(\Gamma)\right\rangle .
$$

We note that $\mathbb{G}$ is not necessarily finitely generated. 
Let $\Gamma^{\prime}=\left(V\left(\Gamma^{\prime}\right), E\left(\Gamma^{\prime}\right)\right)$ be a full subgraph of $\Gamma$. It is not hard to show (see for instance Esyp, the second author and Remeslennikov [24]) that the partially commutative group $\mathbb{G}^{\prime}=\mathbb{G}\left(\Gamma^{\prime}\right)$ is the subgroup of $\mathbb{G}$ generated by $V\left(\Gamma^{\prime}\right)$, ie $\mathbb{G}\left(\Gamma^{\prime}\right)=\left\langle V\left(\Gamma^{\prime}\right)\right\rangle$. Following Duncan, the second author and Remeslennikov [22], we call $\mathbb{G}\left(\Gamma^{\prime}\right)$ a canonical parabolic subgroup of $\mathbb{G}$.

We denote the length of a word $w$ by $|w|$. For a word $w \in \mathbb{G}$, we denote by $\bar{w}$ a geodesic of $w$. Naturally, $|\bar{w}|$ is called the length of an element $w \in \mathbb{G}$. An element $w \in \mathbb{G}$ is called cyclically reduced if the length of $\bar{w}^{2}$ is twice the length of $\bar{w}$ or, equivalently, the length of $w$ is minimal in the conjugacy class of $w$.

For a given word $w$, denote by $\operatorname{alph}(w)$ the set of letters occurring in $w$. For a word $w \in \mathbb{G}$, define $\mathbb{A}(w)$ to be the subgroup of $\mathbb{G}$ generated by all letters that do not occur in a geodesic $\bar{w}$ and commute with $w$. The subgroup $\mathbb{A}(w)$ is well-defined (independent of the choice of a geodesic $\bar{w}$ ); see [24]. Let $v, w \in \mathbb{G}$ be so that $[v, w]=1$ and $\operatorname{alph}(v) \cap \operatorname{alph}(w)=\varnothing$ or, which is equivalent, $v \in \mathbb{A}(w)$ and $w \in \mathbb{A}(v)$. In this case, we say that $v$ and $w$ disjointly commute and write $v \leftrightarrows w$. Given a set of elements $S$ of $\mathbb{G}$, define $\mathbb{A}(S)=\bigcap_{w \in S} \mathbb{A}(w)$.

For a (not necessarily finitely generated) partially commutative group $\mathbb{G}(\Gamma)$, consider its noncommutation graph $\Delta=(V(\Delta), E(\Delta))$ defined as follows. The vertex set $V(\Delta)$ coincides with $V(\Gamma)$. There is an edge connecting $v_{i}$ and $v_{j}$ in $\Delta$ if and only if $i \neq j$ and there is no edge connecting $v_{i}$ and $v_{j}$ in $\Gamma$. Note that the graph $\Delta$ is the complement graph of the graph $\Gamma$. The graph $\Delta$ is a union of its connected components $I_{1}, \ldots, I_{k}$, which induce a decomposition of $\mathbb{G}$ as the direct product

$$
\mathbb{G}=\mathbb{G}\left(I_{1}\right) \times \cdots \times \mathbb{G}\left(I_{k}\right) .
$$

Given $w \in \mathbb{G}$ and the set $\operatorname{alph}(w)$, just as above, consider the graph $\Delta(\operatorname{alph}(w))$ (which is a full subgraph of $\Delta$ ). This graph can be either connected or not. If it is connected, we call $w$ a block. If $\Delta(\operatorname{alph}(w))$ is not connected, then we can decompose $w$ into the product

$$
w=w_{j_{1}} \cdot w_{j_{2}} \cdots w_{j_{t}}, \quad j_{1}, \ldots, j_{t} \in J,
$$

where $|J|$ is the number of connected components of $\Delta(\operatorname{alph}(w))$ and the word $w_{j_{i}}$ is a word in the letters from the $j_{i}^{\text {th }}$ connected component. Clearly, the words $\left\{w_{j_{1}}, \ldots, w_{j_{t}}\right\}$ pairwise disjointly commute. Each word $w_{j_{i}}, i \in 1, \ldots, t$ is a block and so we refer to presentation (1) as the block decomposition of $w$.

Observe that the number of blocks of the block decomposition of $w \in \mathbb{G}$ is bounded above by the rank of $\mathbb{G}$. An element $g \in \mathbb{G}$ is called irreducible if it is a conjugate of a cyclically reduced block element. 
Remark Irreducible elements play a very important role in the theory of partially commutative groups. They are crucial in describing centralisers of elements; they are used to prove that free extensions of centralisers are discriminated by partially commutative groups (see [14]); they are key to understanding the cut-points in the asymptotic cones of partially commutative groups (see Behrstock and Charney [4]); and they will be used essentially in this paper.

An element $w \in \mathbb{G}$ is called a least root (or simply, root) of $v \in \mathbb{G}$ if there exists a positive integer $1<m \in \mathbb{N}$ such that $v=w^{m}$ and there does not exists $w^{\prime} \in \mathbb{G}$ and $1<m^{\prime} \in \mathbb{N}$ such that $w=w^{\prime m^{\prime}}$. In this case, we write $w=\sqrt{v}$. By a result from Duchamp and Krob [21], partially commutative groups have least roots, that is, the root element of $v$ is defined uniquely.

Let $\Gamma$ be a simplicial graph. For any $x \in V(\Gamma)$, define $x^{\perp}$ to be the subset of all vertices $y \in V(\Gamma)$ so that there is an edge $(x, y) \in E(\Gamma)$. We note that $x \notin x^{\perp}$. Given a subset $X \subseteq V(\Gamma)$, set $X^{\perp}=\bigcap_{x \in X} x^{\perp}$.

Let $w$ be a cyclically reduced element of $\mathbb{G}$. It is not hard to see $\left\langle\operatorname{alph}(w)^{\perp}\right\rangle=\mathbb{A}(w)$. Introduce an equivalence relation $\sim$ on the set of vertices $V(\Gamma)$. For two vertices $v_{1}, v_{2} \in V(\Gamma)$, set $v_{1} \sim v_{2}$ if and only if $v_{1}^{\perp}=v_{2}^{\perp}$. Since for every $v \in \Gamma$, we have $v \notin v^{\perp}$, it follows that if $v_{1} \sim v_{2}$, then they are not connected by an edge in $\Gamma$. Define the graph $\Gamma^{\prime}$ whose vertices are $\sim$ equivalence classes and there is an edge joining $[u]$ to $[v]$ if and only if $\left(u^{\prime}, v^{\prime}\right)$ is an edge of $\Gamma$ for some (and thus for all) $u^{\prime} \in[u]$ and $v^{\prime} \in[v]$. The graph $\Gamma^{\prime}$ is called the deflation of $\Gamma$. Observe that the partially commutative group $\mathbb{G}\left(\Gamma^{\prime}\right)$ is isomorphic to a canonical parabolic subgroup of $\mathbb{G}(\Gamma)$.

Definition 2.1 A canonical parabolic subgroup $\mathbb{K}$ of a partially commutative group is called closed if $\mathbb{K}^{\perp \perp}=\mathbb{K}$. The subgroup $\mathbb{K}$ is called coirreducible if $\mathbb{K}$ is closed and its complement $\mathbb{K}^{\perp}$ is a directly indecomposable canonical parabolic subgroup. We denote by $\operatorname{alph}(\mathbb{K})$ the set of canonical generators of $\mathbb{G}$ that generate $\mathbb{K}$.

In our setting, the set of edges $E(\Gamma)$ of the graph $\Gamma$ will be decomposed into a disjoint union of two sets, $E(\Gamma)=E_{d}(\Gamma) \cup E_{c}(\Gamma), E_{d}(\Gamma) \cap E_{c}(\Gamma)=\varnothing$. Let $\mathbb{G}_{d}$ and $\mathbb{G}_{c}$ be the partially commutative groups defined by the graphs $\left(V(\Gamma), E_{d}(\Gamma)\right)$ and $\left(V(\Gamma), E_{c}(\Gamma)\right)$, correspondingly. Then any canonical parabolic subgroup $\mathbb{K}$ of $\mathbb{G}$ naturally defines canonical parabolic subgroups $\mathbb{K}_{d}$ and $\mathbb{K}_{c}$ of $\mathbb{G}_{d}$ and $\mathbb{G}_{c}$, correspondingly.

We say that a canonical parabolic subgroup $\mathbb{K}$ of $\mathbb{G}$ is $E_{d}(\Gamma)$-coirreducible $\left(E_{d}(\Gamma)\right.$ directly (in)decomposable) if so is the induced subgroup $\mathbb{K}_{d}$ of $\mathbb{G}_{d}$. Similarly, a subgroup $\mathbb{K}$ is called $E_{c}(\Gamma)$-abelian if so is the induced subgroup $\mathbb{K}_{c}$ of $\mathbb{G}_{c}$. 
The canonical parabolic subgroup $\mathbb{A}\left(\mathbb{K}_{d}\right)$ of $\mathbb{G}_{d}$ naturally defines a canonical parabolic subgroup of $\mathbb{G}$, which we shall denote by $\mathbb{A}_{E_{d}(\Gamma)}\left(\mathbb{K}_{d}\right)$.

The remainder of this section is devoted to defining a normal form for partially commutative groups introduced in [20] by Diekert and Muscholl. The main feature of this normal form is that it is "stable under inversions", that is if the word $w$ is in the normal form, then so is its inverse $w^{-1}$. This normal form is only used to prove some of the more technical statements of the paper. Hence, the rest of this section can be omitted at a first reading.

Let $\mathbb{G}$ be a partially commutative group given by the presentation $\langle\mathcal{A} \mid R\rangle$. Let $\mathbb{F}=\mathbb{F}\left(\mathcal{A}^{ \pm 1}\right)$ be the free monoid on the alphabet $\mathcal{A} \cup \mathcal{A}^{-1}$ and let $\mathbb{T}=\mathbb{T}\left(\mathcal{A}^{ \pm 1}\right)$ be the partially commutative monoid with involution given by the presentation

$$
\mathbb{T}\left(\mathcal{A}^{ \pm 1}\right)=\left\langle\mathcal{A} \cup \mathcal{A}^{-1} \mid R_{\mathbb{T}}\right\rangle,
$$

where $\left[a_{i}^{\epsilon}, a_{j}^{\delta}\right] \in R_{\mathbb{T}}$ if and only if $\left[a_{i}, a_{j}\right] \in R, \epsilon, \delta \in\{-1,1\}$ (recall that, by definition, $\left.\left[a_{i}, a_{i}\right] \notin R\right)$. The involution on $\mathbb{T}$ is induced by the operation of inversion in $\mathbb{G}$ and does not have fixed points. We refer to it as to the inversion in $\mathbb{T}$ and denote it by ${ }^{-1}$.

Following [20], we define a clan to be a maximal subset $C=\mathcal{C} \cup \mathcal{C}^{-1}$ of $\mathcal{A} \cup \mathcal{A}^{-1}$ such that $[a, c] \notin R_{\mathbb{T}}$ if and only if $[b, c] \notin R_{\mathbb{T}}$ for all $a, b \in C$ and $c \in \mathcal{A}^{ \pm 1}$. A clan $C$ is called thin if there exist $a \in C$ and $b \in \mathcal{A}^{ \pm 1} \backslash C$ such that $[a, b] \in R_{\mathbb{T}}$ and is called thick, otherwise.

Example 2.2 If $\mathbb{T}$ is a direct product of $d$ free monoids, then the number of thin clans is $d$ for $d>1$, and it is 0 for $d=1$.

Let $\mathbb{G}=\langle a, b, c, d, e \mid[a, c],[c, b],[b, d],[d, a],[c, d]\rangle$, then $\left\{a, a^{-1}, b, b^{-1}\right\},\left\{c, c^{-1}\right\}$, $\left\{d, d^{-1}\right\}$ are the thin clans of $\mathbb{T}$ and $\left\{e, e^{-1}\right\}$ is the thick clan (note that $c$ and $d$ do not belong to the same clan, since $[c, d] \in R_{\mathbb{T}}$, but $[c, c] \notin R_{\mathbb{T}}$.

It follows from the definition that there is at most one thick clan and that the number of thin clans never equals 1 . Every element of $\mathcal{A} \cup \mathcal{A}^{-1}$ belongs to exactly one clan. In the following, we pick a thin clan and we make it thick by removing commutation. It might be that the number of clans does not change, but the number of thin clans decreases. This is the reason why the definition of DM-normal form below is based on thin clans (instead of considering all clans).

It is convenient to encode an element of the partially commutative monoid as a finite labelled acyclic oriented graph $[V, E, \lambda]$, where $V$ is the set of vertices, $E$ is the set of edges and $\lambda: V \rightarrow \mathcal{A}^{ \pm 1}$ is the labelling. Such a graph induces a labelled partial order. For an element $w \in \mathbb{T}, w=b_{1} \cdots b_{n}, b_{i} \in \mathcal{A}^{ \pm 1}$, we introduce the graph $[V, E, \lambda]$ 
as follows. The set of vertices of $[V, E, \lambda]$ is in one-to-one correspondence with the letters of $w, V=\{1, \ldots, n\}$. For the vertex $j$ we set $\lambda(j)=b_{j}$. We define an edge from $b_{i}$ to $b_{j}$ if and only if both $i<j$ and $\left[b_{i}, b_{j}\right] \notin R_{\mathbb{T}}$. The graph $[V, E, \lambda]$ thereby obtained is called the dependence graph of $w$. Up to isomorphism, the dependence graph of $w$ is unique, and so is its induced labelled partial order, which we further denote by $[V, \leq, \lambda]$.

Let $c_{1}<\cdots<c_{q}$ be the linearly ordered subset of $[V, \leq, \lambda]$ containing all vertices with label in the clan $C$. For the vertex $v \in V$, we define the source point $s(v)$ and the target point $t(v)$ as

$$
s(v)=\sup \left\{i \mid c_{i} \leq v\right\}, \quad t(v)=\inf \left\{i \mid v \leq c_{i}\right\} .
$$

By convention, $\sup \varnothing=0$ and $\inf \varnothing=q+1$. Thus, $0 \leq s(v) \leq q, 1 \leq t(v) \leq q+1$ and $s(v) \leq t(v)$ for all $v \in V$. Note that we have $s(v)=t(v)$ if and only if the label of $v$ belongs to $C$.

For $0 \leq s \leq t \leq q+1$, we define the median position $m(s, t)$ as follows. For $s=t$, we let $m(s, t)=s$. For $s<t$, by [20, Lemma 1], there exist unique $l$ and $k$ such that $s \leq l<t, k \geq 0$ and

$$
c_{s+1} \cdots c_{l} \in \mathbb{F}(\mathcal{C})\left(\mathcal{C}^{-1} \mathbb{F}(\mathcal{C})\right)^{k}, \quad c_{l+1} \cdots c_{t-1} \in\left(\mathbb{F}\left(\mathcal{C}^{-1}\right) \mathcal{C}\right)^{k} \mathbb{F}\left(\mathcal{C}^{-1}\right),
$$

where $\mathbb{F}(\mathcal{C})$ and $\mathbb{F}\left(\mathcal{C}^{-1}\right)$ are free monoids on the indicated alphabets. Then we define $m(s, t)=l+\frac{1}{2}$ and we call $m(s, t)$ the median position. Define the global position of $v \in V$ to be $g(v)=m(s(v), t(v))$.

We define the normal form $\operatorname{nf}(w)$ of an element $w \in \mathbb{T}$ by introducing new edges into the dependence graph $[V, E, \lambda]$ of $w$. Let $u, v \in V$ be such that $\lambda(v) \in C$ and $[\lambda(u), \lambda(v)] \in R_{\mathbb{T}}$. We define a new edge from $u$ to $v$ if $g(u)<g(v)$, otherwise, we define a new edge from $v$ to $u$. The new dependence graph $[V, \widehat{E}, \lambda]$ defines a unique element of the trace monoid $\widehat{\mathbb{T}}$, where $\widehat{\mathbb{T}}$ is obtained from $\mathbb{T}$ by omitting the commutativity relations of the form $[c, a]$ for any $c \in C$ and any $a \in \mathcal{A}^{ \pm 1}$. Note that the number of thin clans of $\widehat{\mathbb{T}}$ is strictly less than the number of thin clans of $\mathbb{T}$. We proceed by designating a thin clan in $\widehat{\mathbb{T}}$ and introducing new edges in the dependence graph $[V, \widehat{E}, \lambda]$.

It is proven in [20, Lemma 4], that the normal form $\mathrm{nf}$ is a map from the trace monoid $\mathbb{T}$ to the free monoid $\mathbb{F}\left(\mathcal{A} \cup \mathcal{A}^{-1}\right)$, which is compatible with inversion, ie it satisfies that $\pi(\operatorname{nf}(w))=w$ and $\operatorname{nf}\left(w^{-1}\right)=\operatorname{nf}(w)^{-1}$, where $w \in \mathbb{T}$ and $\pi$ is the canonical epimorphism from $\mathbb{F}\left(\mathcal{A} \cup \mathcal{A}^{-1}\right)$ to $\mathbb{T}$.

We refer to this normal form as to the DM-normal form or simply as to the normal form of an element $w \in \mathbb{T}$. 
Let $w \in \mathbb{G}$ be a word and let $\mathbb{H}<\mathbb{G}$ be a canonical parabolic subgroup, that is, $\mathbb{H}=\mathbb{H}_{1} \times \mathbb{H}_{2}, \mathbb{H}_{i} \neq 1$. We say that $w$ has $2 k-1 \mathbb{H}$-alternations if $w$ contains a subword $v_{1} u_{1} \cdots v_{k} u_{k}$, where $v_{i} \in \mathbb{H}_{1}$ and $u_{i} \in \mathbb{H}_{2}$ are nontrivial words, $i=1, \ldots, k$.

Lemma 2.3 Let $w \in \mathbb{G}$ be written in the DM-normal form. Let $\mathbb{H}<\mathbb{G}$ be a canonical parabolic subgroup which decomposes as a nontrivial direct product of two canonical parabolic subgroups $\mathbb{H}_{1}$ and $\mathbb{H}_{2}, \mathbb{H}=\mathbb{H}_{1} \times \mathbb{H}_{2}$. Then the number of $\mathbb{H}$-alternations in $w$ is bounded above by a constant that depends only on the number of clans of $\mathbb{G}$.

Proof We use induction on the number of thin clans of $\mathbb{G}$. If the number of thin clans equals one, then the statement is obvious.

Suppose that the statement is true for all partially commutative groups with less than $n$ thin clans and let $\mathbb{G}$ have precisely $n$ thin clans. Let $w \in \mathbb{G}$ be some word, let $[V, E, \lambda]$ be the dependence graph of $w$ and let $C$ be a thin clan of $\mathbb{G}$, and write

$$
w=w_{1} c_{1} w_{2} \cdots w_{q} c_{q} w_{q+1},
$$

where $c_{1}, \ldots, c_{q}$ are all the letters in $w$ which belong to the clan $C$ and the dependence relations for $C$ have already been established.

By definition of the DM-normal form,

$$
\operatorname{nf}(w)=\operatorname{nf}\left(w_{1}\right) c_{1} \operatorname{nf}\left(w_{2}\right) \cdots \operatorname{nf}\left(w_{q}\right) c_{q} \operatorname{nf}\left(w_{q+1}\right) .
$$

Therefore, if $\mathbb{H} \cap\langle C\rangle=1$, then the statement follows by induction.

Suppose $\mathbb{H}$ and $\langle C\rangle$ intersect nontrivially, then either $\mathbb{H} \cap\langle C\rangle<\mathbb{H}_{1}$ or $\mathbb{H} \cap\langle C\rangle<\mathbb{H}_{2}$. Let $v_{1} \cdots v_{k}$ be an $\mathbb{H}$-alternation in $\operatorname{nf}(w)$. If $v_{1} \cdots v_{k}$ does not contain $c_{i}$ for all $i=1, \ldots, q$, then the statement follows by induction. Let us assume that $v_{1} \cdots v_{k}$ contains $c_{i}$ for some $i=1, \ldots, q$ and let $i$ be minimal so that $c_{i}$ is a letter of $v_{j}$.

If $j=k$, then the bound on $k$ follows by induction. Suppose that $j \neq 1$, then $v_{j-1}$ is a subword of $\operatorname{nf}\left(w_{i}\right)$ and $v_{j+1}$ is a subword of $\operatorname{nf}\left(w_{l}\right)$ for some $l \neq i$. But since $v_{j-1} \leftrightarrows v_{j}$ and $v_{j+1} \leftrightarrows v_{j}$, it follows that for every letters $x$ and $y$ from $v_{j-1}$ and $v_{j+1}$, the global positions $g(x)$ and $g(y)$ (with respect to $C$ ) coincide, contradicting the definition of the DM-normal form. It follows that $k \neq 2, \ldots, k-1$.

Suppose that $j=1$. Let $i^{\prime}$ be minimal so that $i^{\prime}>i$ and $c_{i^{\prime}}$ is contained in $v_{1} \cdots v_{k}$. Observe that, without loss of generality, we may assume that such $i^{\prime}$ exists since, otherwise, the bound on $k$ follows by induction. The letter $c_{i^{\prime}}$ is a letter of some $v_{j^{\prime}}$. If $j^{\prime} \neq k$, then $v_{j^{\prime}-1}$ is a subword of $w_{i^{\prime}}$ and $v_{j^{\prime}+1}$ is a subword of $\operatorname{nf}\left(w_{l^{\prime}}\right)$ for some $l^{\prime}>i^{\prime}$. But, since $v_{j^{\prime}-1} \leftrightarrows v_{j^{\prime}}$ and $v_{j^{\prime}+1} \leftrightarrows v_{j^{\prime}}$, it follows that for every letters $x$ and $y$ from $v_{j^{\prime}-1}$ and $v_{j^{\prime}+1}$, the global positions $g(x)$ and $g(y)$ (with respect to $C$ ) 
coincide, which contradict the definition of the DM-normal form. If $j^{\prime}=k$, then the bound on $k$ follows by induction.

\subsection{Algebraic geometry over groups}

The objective of this section is to establish the basics of algebraic geometry over groups. We refer the reader to [3; 19] for details. Let $G=\langle A\rangle$ be a group and $F(X)$ be the free group on the alphabet $X, X=\left\{x_{1}, x_{2}, \ldots, x_{n}\right\}$. Denote by $G[X]$ the free product $G * F(X)$.

For any element $s \in G[X]$, the formal equality $s=1$ can be treated, in an obvious way, as an equation over $G$. In general, for a subset $S \subset G[X]$, the formal equality $S=1$ can be treated as a system of equations over $G$ with coefficients in $A$. Elements from $X$ are called variables and elements from $A^{ \pm 1}$ are called coefficients or constants. To emphasise this we sometimes write $S(X, A)=1$.

A solution $U$ of the system $S(X)=1$ over a group $G$ is a tuple of elements $g_{1}, \ldots, g_{n} \in G$ such that every equation from $S$ vanishes at $\left(g_{1}, \ldots, g_{n}\right)$, that is, $S_{i}\left(g_{1}, \ldots, g_{n}\right)=1$ in $G$, for all $S_{i} \in S$. Equivalently, a solution $U$ of the system $S=1$ over $G$ is a $G$-homomorphism $\pi_{U}: G[X] \rightarrow G$ induced by the map $\pi_{U}: x_{i} \mapsto g_{i}$ such that $S \subseteq \operatorname{ker}\left(\pi_{U}\right)$. When no confusion arises, we abuse the notation and write $U(w)$, where $w \in G[X]$, instead of $\pi_{U}(w)$.

Denote by $\operatorname{ncl}\langle S\rangle$ the normal closure of $S$ in $G[X]$. Then every solution of $S(X)=1$ in $G$ gives rise to a $G$-homomorphism $G[X] / \operatorname{ncl}\langle S\rangle \rightarrow G$, and vice versa. The set of all solutions over $G$ of the system $S=1$ is denoted by $V_{G}(S)$ and is called the algebraic set or variety defined by $S$.

For every system of equations $S$, we set the radical of the system $S$ to be the following subgroup of $G[X]$ :

$$
R(S)=\left\{T(X) \in G[X] \mid \forall g_{1}, \ldots, \forall g_{n}\left(S\left(g_{1}, \ldots, g_{n}\right)=1 \rightarrow T\left(g_{1}, \ldots, g_{n}\right)=1\right)\right\} .
$$

It is easy to see that $R(S)$ is a normal subgroup of $G[X]$ that contains $S$. There is a one-to-one correspondence between algebraic sets $V_{G}(S)$ and radical subgroups $R(S)$ of $G[X]$. Notice that if $V_{G}(S)=\varnothing$, then $R(S)=G[X]$.

It follows from the definition that

$$
R(S)=\bigcap_{U \in V_{G}(S)} \operatorname{ker}\left(\pi_{U}\right)
$$

The quotient group

$$
G_{R(S)}=G[X] / R(S)
$$


is called the coordinate group of the algebraic set $V_{G}(S)$ (or of the system $S$ ). There exists a one-to-one correspondence between algebraic sets and coordinate groups. More formally, the categories of algebraic sets and coordinate groups are dual; see [3, Theorem 4]. If a group $G=G_{R(S)}$ is given as the quotient $G[X] / R(S)$, we say that $G$ is given by its radical presentation. If the corresponding system $S$ is finite, we say that $G$ is given by a finite radical presentation.

Given a system of equations $S=1$, we denote by $G_{S}$ the group $G[X] / \operatorname{ncl}\langle S\rangle$.

A group $H$ is called ( $G$-)equationally Noetherian if every system $S(X)=1$ with coefficients from $G$ is equivalent over $G$ to a finite subsystem $S_{0}=1$, where $S_{0} \subset S$, ie the system $S$ and its subsystem $S_{0}$ define the same algebraic set. If $G$ is $G-$ equationally Noetherian, then we say that $G$ is equationally Noetherian. If $G$ is equationally Noetherian then the Zariski topology over $G^{n}$ is Noetherian for every $n$, ie every proper descending chain of closed sets in $G^{n}$ is finite. This implies that every algebraic set $V$ in $G^{n}$ is a finite union of irreducible subsets, called irreducible components of $V$, and such a decomposition of $V$ is unique. Recall that a closed subset $V$ is irreducible if it is not a union of two proper closed (in the induced topology) subsets.

We note that partially commutative groups are linear (see [33]), thus, equationally Noetherian (see [3]).

We say that a family of homomorphisms $\left\{\varphi_{i}\right\} \subset \operatorname{Hom}(H, K)$ separates (discriminates) $H$ into $K$ if for every nontrivial element $h \in H$ (every finite set of nontrivial elements $\left.H_{0} \subset H\right)$ there exists $k$ so that $\varphi_{k}(h) \neq 1\left(\varphi_{k}(h) \neq 1\right.$ for every $\left.h \in H_{0}\right)$. In this case, we also say that $H$ is residually $K$ (that $H$ is fully residually $K$ ) and call the family $\left\{\varphi_{i}\right\}$ separating (discriminating).

Remark 2.4 There is a natural epimorphism from $G[X] / \operatorname{ncl}\langle S\rangle$ onto $G_{R(S)}$. This epimorphism is an isomorphism if and only if $G[X] / \operatorname{ncl}\langle S\rangle$ is residually $G$.

Theorem 2.5 [47] Let $H$ be a group. Then for a finitely generated group $G$ the following conditions are equivalent:

(1) $G$ is fully residually $H$.

(2) $G$ is the coordinate group of an irreducible variety over $H$.

If any of the above two conditions holds, then

(3) $G$ embeds into an ultrapower of $H$.

Furthermore if $H$ is equationally Noetherian, then all three conditions above are equivalent. 
Lemma 2.6 Let $G$ be a fully residually $H$ group and let $\left\{\varphi_{i}\right\}$ be a discriminating family for $G$. If the family $\left\{\varphi_{i}\right\}$ is a union of finitely many families, $\left\{\varphi_{i}\right\}=\left\{\varphi_{i, 1}\right\} \cup$ $\cdots \cup\left\{\varphi_{i, n}\right\}$, then one of the families $\left\{\varphi_{i, k}\right\}$ is a discriminating family for $G$.

Proof Without loss of generality, we may assume that $n=2$ and $\left\{\varphi_{i}\right\}=\left\{\phi_{i}\right\} \cup\left\{\psi_{i}\right\}$. We show that either $\left\{\phi_{i}\right\}$ or $\left\{\psi_{i}\right\}$ is a discriminating family for $G$.

If we assume the contrary, then there exist finite sets of nontrivial elements $S=$ $\left\{g_{1}, \ldots, g_{m}\right\} \subset G$ and $T=\left\{h_{1}, \ldots, h_{k}\right\} \subset G$ which can not be discriminated into $H$ by $\left\{\phi_{i}\right\}$ and $\left\{\psi_{i}\right\}$, correspondingly, ie for all $i$ we have $\psi_{i}\left(g_{l_{i}}\right)=1$ and $\phi_{i}\left(h_{m_{i}}\right)=1$ for some $l_{i}=1, \ldots, m, m_{i}=1, \ldots, k$. Since $\left\{\varphi_{i}\right\}=\left\{\phi_{i}\right\} \cup\left\{\psi_{i}\right\}$, the set $S \cup T$ can not be discriminated by $\left\{\varphi_{i}\right\}$ into $H$, a contradiction.

Remark 2.7 Let $G$ be a group discriminated by a finitely generated partially commutative group $\mathbb{G}$. Notice that since $\mathbb{G}$ has only finitely many different canonical parabolic subgroups, then, by Lemma 2.6, for any $H<G$, there exists a canonical parabolic subgroup $\mathbb{H}$ of $\mathbb{G}$ with the two following properties: there exists a discriminating family $\left\{\varphi_{i}\right\}$ such that $\varphi_{i}(H)<\mathbb{H}$ and there exist no proper canonical parabolic subgroups $\mathbb{H}^{\prime}$ of $\mathbb{H}$ with the first property.

The term limit group was introduced by Sela in [52] in the setting of free groups. The original definition is given in terms of the action of $G$ on a limiting real tree. One can prove (see [52]) that, in the case of free groups, the geometric and residual definitions are equivalent. In Section 9, following Sela, we introduce the class of (geometric) limit groups over partially commutative groups and show that it coincides with the class of finitely generated fully residually partially commutative groups. From now on, slightly abusing the terminology, we sometimes refer to finitely generated fully residually $\mathbb{G}$ groups as limit groups over $\mathbb{G}$.

\section{Tribal Makanin-Razborov diagrams}

In the free group case, Kharlampovich and Miasnikov used induction on the height of the Makanin-Razborov diagram and the structure of groups assigned to its vertices to give a hierarchical construction of NTQ-groups or $\omega$-residually free towers; see [38]. Our aim is to use a similar approach in order to construct a natural generalisation of towers of free groups, which we call graph towers.

In [14] we described an analogue of the Makanin-Razborov process for partially commutative groups, which, given a finitely generated group $G$ and a partially commutative group $\mathbb{G}$, produces a diagram that encodes the set of all homomorphisms from $G$ 
to $\mathbb{G}$. In order to construct graph towers the only missing ingredient is to have a good understanding of the structure of coordinate groups associated to the vertices of the diagram.

To describe the structure of coordinate groups associated to vertices in the MakaninRazborov diagrams for free groups, one only needs to change the presentation of the group to the one that clearly exhibits its algebraic structure as amalgamated product or HNN-extension.

Example 3.1 Assume that the coordinate group associated to a vertex of linear type has the presentation

$G=\left\langle x_{1}, \ldots, x_{n} \mid w_{1}\left(x_{3}, \ldots, x_{n}\right)=x_{1} x_{2}, w_{2}\left(x_{3}, \ldots, x_{n}\right)=1, \ldots, w_{k}\left(x_{3}, \ldots, x_{n}\right)=1\right\rangle$,

ie the generators $x_{1}$ and $x_{2}$ only appear once in a single relation. In this case, using Tietze transformation, we can change the presentation of the group and show that it splits as a free product, that is,

$$
G=\left\langle x_{3}, \ldots, x_{n} \mid w_{2}\left(x_{3}, \ldots, x_{n}\right)=1, \ldots, w_{k}\left(x_{3}, \ldots, x_{n}\right)=1\right\rangle *\left\langle x_{2}\right\rangle .
$$

Unfortunately, in the case of partially commutative groups the presence of commutation constraints in the presentation prevents one from using this direct approach. For instance, suppose that we add the commutation constraints $\left[x_{1}, x_{3}\right]$ and $\left[x_{2}, x_{3}\right]$ in the presentation of $G$, ie

$$
\begin{array}{r}
H=\left\langle x_{1}, \ldots, x_{n}\right| w_{1}\left(x_{3}, \ldots, x_{n}\right)=x_{1} x_{2}, \ldots, w_{k}\left(x_{3}, \ldots, x_{n}\right)=1, \\
\left.\left[x_{1}, x_{3}\right]=1,\left[x_{2}, x_{3}\right]=1\right\rangle .
\end{array}
$$

This could very well be the coordinate group associated to a vertex of linear type in our diagram, however there is no obvious way of changing its presentation to exhibit any splitting.

This shows that, unlike in the free group case, in the partially commutative case coordinate groups associated to vertices of the diagram do not necessarily split as amalgamated products or HNN-extensions. To overcome this main difficulty, our goal is to show that if a coordinate group associated to a vertex has "nontrivial dynamics", that is, there is a nontrivial group of automorphisms associated to it in the diagram, then it does split. In order to pursue this approach, one needs to perform a very careful analysis of the dynamics of infinite branches in the process presented in [14] taking into consideration the behaviour not only of the relations of the presentation but also, crucially, of the commutation constraints. 
To this end, in this section we review and slightly modify the process described in [14] to obtain a new diagram, called the tribal solution tree. Just as the solution tree constructed in [14], the tribal solution tree encodes the set of all homomorphisms from a finitely generated group to a partially commutative group; however, the set of commutation constraints of the coordinate groups associated to its vertices (called tribal coordinate groups) has more structure. In the next section we shall show that tribal coordinate groups have a clear algebraic structure and split as amalgamated products.

The process to construct the solution tree presented in [14] is very long and rather involved and so we do not repeat the full construction here. Therefore, this section is not self-contained: we frequently refer to [14] for details and some familiarity with that text is assumed.

We now begin by reviewing some of the constructions presented in [14]. As shown in [14, Chapter 3], given a finitely generated group $G$ and a partially commutative group $\mathbb{G}$, one can effectively construct finitely many constrained generalised equations $\Omega_{0}, \ldots, \Omega_{m}$ (see definition below) and homomorphisms $\pi_{i}$ from $G$ to the coordinate group of the generalised equation $\Omega_{i}$ so that any homomorphism from $G$ to $\mathbb{G}$ factors through the coordinate group of a generalised equation $\Omega_{i}$ for some $i=0, \ldots, m$, ie for any homomorphism $\varphi: G \rightarrow \mathbb{G}$, there exist $i$ and a homomorphism $\varphi^{\prime}: G_{R\left(\Omega_{i}\right)} \rightarrow \mathbb{G}$ so that $\varphi=\pi_{i} \varphi^{\prime}$.

A generalised equation $\Upsilon$ can be viewed as a combinatorial object which encodes a system of equations over a free monoid.

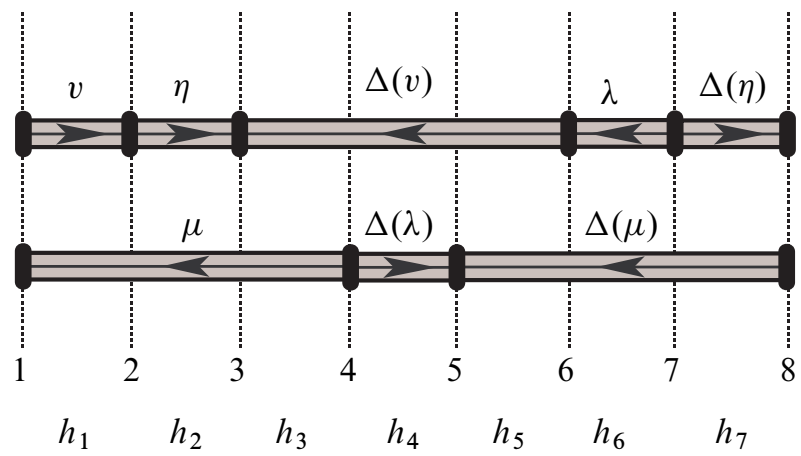

Figure 1: Simple quadratic generalised equation

Items $h_{i}$ correspond to variables and each pair of dual bases $\mu, \Delta(\mu)$ defines the equation $\left(h_{\alpha(\mu)} \cdots h_{\beta(\mu)-1}\right)^{\varepsilon(\mu)}=\left(h_{\alpha(\Delta(\mu))} \cdots h_{\beta(\Delta(\mu))-1}\right)^{\varepsilon(\Delta(\mu))}$. A tuple $H=$ $\left(H_{1}, \ldots, H_{\rho \Upsilon}\right)$ is a solution of the generalised equation $\Upsilon$ if $H_{\alpha(\mu)} \cdots H_{\beta(\mu)-1}$ and 
$H_{\alpha(\Delta(\mu))} \cdots H_{\beta(\Delta(\mu))-1}$ are freely reduced words so that $\left(H_{\alpha(\mu)} \cdots H_{\beta(\mu)-1}\right)^{\varepsilon(\mu)}$ is graphically equal to $\left(H_{\alpha(\Delta(\mu))} \cdots H_{\beta(\Delta(\mu))-1}\right)^{\varepsilon(\Delta(\mu))}$, for every pair of dual bases $\mu, \Delta(\mu)$; see [14, Chapter 3] for details.

A constrained generalised equation $\Omega=\langle\Upsilon, \Re \Upsilon\rangle$ is a generalised equation $\Upsilon$ together with a set of commutation constraints $\Re \Upsilon \subseteq h \times h$ associated to the variables (items) $h$ of $\Upsilon$. A tuple $H=\left(H_{1}, \ldots, H_{\rho_{\Omega}}\right)$ is a solution of the generalised equation $\Omega$ if $H_{\alpha(\mu)} \cdots H_{\beta(\mu)-1}$ and $H_{\alpha(\Delta(\mu))} \cdots H_{\beta(\Delta(\mu))-1}$ are geodesic words in $\mathbb{G}$ so that $\left(H_{\alpha(\mu)} \cdots H_{\beta(\mu)-1}\right)^{\varepsilon(\mu)}$ is graphically equal to $\left(H_{\alpha(\Delta(\mu))} \cdots H_{\beta(\Delta(\mu))-1}\right)^{\varepsilon(\Delta(\mu))}$ for every pair of dual bases $\mu, \Delta(\mu)$, and $H$ satisfies the commutation constraints, ie $H_{i} \leftrightarrows H_{j}$ for all commutation constraints $\Re_{\Upsilon}\left(h_{i}, h_{j}\right)$

The coordinate group $G_{R(\Upsilon)}$ of $\Upsilon$ is the group $\mathbb{G}[h] / R(\Upsilon)$, where, abusing the notation, we denote by $\Upsilon$ the system of equations associated to the generalised equation $\Upsilon$. The coordinate group $G_{R(\Omega)}$ of $\Omega$ is the group $\mathbb{G}[h] / R(\Omega)$, where, abusing the notation, we denote by $\Omega$ the system of equations associated to the generalised equation $\Upsilon$ and the set of commutation relations $\left[h_{i}, h_{j}\right]$ for all $h_{i}, h_{j}$ so that $\Re_{\Upsilon}\left(h_{i}, h_{j}\right)$. We denote by $G_{\Upsilon}$ (by $G_{\Omega}$ ) the group $\mathbb{G}[h] / \operatorname{ncl}\langle\Upsilon\rangle$ (the group $\mathbb{G}[h] / \operatorname{ncl}\langle\Omega\rangle)$.

The description of the set of homomorphisms $\operatorname{Hom}(G, \mathbb{G})$ reduces to the study of the sets of solutions of the constrained generalised equations $\Omega_{i}, i=0, \ldots, m$.

In short, given a generalised equation $\Omega$, we described a process that produces a (possibly) infinite, locally finite, rooted tree $T(\Omega)$ which encodes the solution set of $\Omega$. Infinite branches of this tree are of three specific types: linear, quadratic or general type. The dynamics of these infinite branches determine specific families of automorphisms of the coordinate group associated to $\Omega$. One then shows that, using these families of automorphisms, one can produce a finite tree, the solution tree $T_{\text {sol }}(\Omega)$ that encodes the set of solutions of $\Omega$; see [14, Theorem 9.2].

We now review and refine this process to obtain a tight control on the behaviour of constraints in infinite branches. We begin by slightly generalising the notion of (constrained) generalised equation. To each (constrained) generalised equation $\Omega=\langle\Upsilon, \Re \Upsilon\rangle$ we associate the following additional information. To each item $h_{i}$ of $\Omega$ we assign a canonical parabolic subgroup of $\mathbb{G}$, denoted by alph $\left(h_{i}\right)$, which is consistent with the constraints, ie if $\left(h_{i}, h_{j}\right) \in \Re \Upsilon$ then alph $\left(h_{i}\right) \leftrightarrows$ alph $\left(h_{j}\right)$. A solution of a generalised equation with associated parabolic subgroups alph $\left(h_{i}\right)$ is a solution of the generalised equation $\Omega$ that further satisfies $\left\langle\operatorname{alph}\left(H_{i}\right)\right\rangle=\operatorname{alph}\left(h_{i}\right)$ for all items $h_{i}$ of $\Omega$.

Given a base $\mu$ (or a section $\sigma=[k, l])$ of $\Omega$, we define $\operatorname{alph}(\mu)(\operatorname{alph}(\sigma))$ as the canonical parabolic subgroup of $\mathbb{G}$ defined by the union of subgroups $\operatorname{alph}\left(h_{i}\right)$ for 
each $i=\alpha(\mu), \ldots, \beta(\mu)-1(i=k, \ldots, l-1)$, ie the $\operatorname{subgroup} \operatorname{alph}(\mu)(\operatorname{alph}(\sigma))$ is the canonical parabolic subgroup generated by the union of the alphabets of the items covered by the base $\mu$ (belonging to the section $\sigma$ ).

Given a finitely generated partially commutative group $\mathbb{G}$ and a generalised equation $\Omega$, there exist finitely many constrained generalised equations with associated parabolic subgroups alph $\left(h_{i}\right)$, say $\left\{\Omega_{1}, \ldots, \Omega_{k}\right\}$ so that every solution $H$ of $\Omega$ is a solution of one generalised equation $\Omega_{i}, i=1, \ldots, k$. Furthermore, it follows from the definition that any solution of $\Omega_{i}$ is a solution of $\Omega$. Hence the study of solutions of a constrained generalised equation is equivalent to the study of solutions of generalised equations with associated canonical parabolic subgroups.

The process which is described in [14, Chapter 4], uses 5 elementary transformations ET1, .., ET5 which, applied to a given (constrained) generalised equation, produce finitely many new ones. In what follows, we use the description of the transformations as well as the notation given in [14, Section 4.2]. For all transformations ET1-ET4, and also ET5 in the case when ET5 does not introduce new boundaries, there is a natural one-to-one correspondence between items of the original generalised equation and items of each of the produced generalised equations. Using this natural correspondence between items, we transfer the associated canonical parabolic subgroups and so we use these transformations for (constrained) generalised equations with associated parabolic subgroups. Suppose that the transformation ET5 introduces a new boundary $q^{\prime}$ between the boundaries $q$ and $q+1$ and a new boundary connection $\left(p, \mu, q^{\prime}\right)$. Let $\Omega^{\prime}$ be the obtained generalised equation. From $\Omega^{\prime}$ we construct finitely many generalised equations with associated parabolic subgroups so that

- $\operatorname{alph}\left(h_{i}^{\prime}\right)=\operatorname{alph}\left(h_{i}\right), i \neq q, q^{\prime}$,

- $\operatorname{alph}\left(h_{q}^{\prime}\right), \operatorname{alph}\left(h_{q^{\prime}}^{\prime}\right)<\operatorname{alph}\left(h_{q}\right)$ so that $\left\langle\operatorname{alph}\left(h_{q}^{\prime}\right) \cup \operatorname{alph}\left(h_{q^{\prime}}^{\prime}\right)\right\rangle=\operatorname{alph}\left(h_{q}\right)$.

The new elementary transformation ET5 (of generalised equations with associated parabolic subgroups) is obtained by applying the transformation ET5 from [14] and then replacing each generalised equation $\Omega^{\prime}$ by finite family generalised equations with associated parabolic subgroups described above.

From now on we abuse the terminology and we call (constrained) generalised equations with associated parabolic subgroups alph $\left(h_{i}\right)$ simply (constrained) generalised equations.

\subsection{Tribes}

In this section we introduce the notion of a tribe which plays a crucial role in the study of the dynamics of infinite branches. The importance of this notion is that it is 
intrinsically related to the fact that one can define a certain finitary "hierarchy" (finite partial order) on the commutation constraints associated to the variables, which, on the one hand, serves as a measure of complexity of the constraints and, on the other hand, it assures that (the normal subgroup generated by) the commutation relations of a given class is invariant under certain automorphisms of the free group. This invariance is a key point that allows us to establish the structure of tribal coordinate groups in Section 4.

For instance, tribes will assure that the group $H$ from Example 3.1 is not a vertex of our diagram, as it is not tribal (see Definition 3.28). However, in the group

$$
\begin{array}{r}
H=\left\langle x_{1}, \ldots, x_{7}\right| x_{3} x_{4} x_{3} x_{4}^{-1}=x_{1} x_{2}, \ldots, w_{k}\left(x_{3}, \ldots, x_{7}\right),\left[x_{i}, x_{5}\right]=1,\left[x_{4}, x_{7}\right]=1, \\
i=1,2,3,4\rangle,
\end{array}
$$

the variables $x_{1}$ and $x_{2}$ belong to the same tribe and the automorphism of the free group induced by the map $x_{1} \rightarrow x_{1} x_{2}^{-1}$ and $x_{i} \rightarrow x_{i}$ for $i \neq 1$ fixes the normal subgroup generated by the commutation relations $\left\{\left[x_{i}, x_{5}\right],\left[x_{4}, x_{7}\right], i=1,2,3,4\right\}$. Using this automorphism, one can rewrite the presentation of $H$ so that it exhibits a splitting as an HHN-extension with stable letter $x_{2}$ and associated subgroup $x_{5}$. This shows the essence of tribes.

As a final remark we stress the importance of the fact that hierarchy be finite. In our case, this is an immediate consequence of the fact that the underlying partially commutative group $\mathbb{G}$ is finitely generated.

Let $\left\{\mathbb{A}\left(S_{1}\right), \ldots, \mathbb{A}\left(S_{\mathfrak{m}}\right)\right\}$ be the finite set of distinct canonical parabolic subgroups of $\mathbb{G}=\langle\mathcal{A}\rangle$, where $S_{i} \subset \mathcal{A}, i=1, \ldots, \mathfrak{m}$.

Let $\Omega=\langle\Upsilon, \Re \Upsilon\rangle$ be a generalised equation (with associated parabolic subgroups $\operatorname{alph}\left(h_{i}\right)$ ). A tribe of $\Omega$ is a set of items, bases and sections of $\Omega$. We say that an item $h_{j}$ (or a base, or a section) of $\Omega$ belongs to the tribe $t_{i}$ if and only if one has that $\mathbb{A}\left(\operatorname{alph}\left(h_{j}\right)\right)=\mathbb{A}\left(S_{i}\right)\left(\right.$ or $\mathbb{A}(\operatorname{alph}(\mu))=\mathbb{A}\left(S_{i}\right)$, or $\mathbb{A}(\operatorname{alph}(\sigma))=\mathbb{A}\left(S_{i}\right)$, correspondingly), for some $i=1, \ldots, \mathfrak{m}$. Notice that, in general, two items $h_{j}, h_{k}$ might belong to the same tribe although $\operatorname{alph}\left(h_{j}\right) \neq \operatorname{alph}\left(h_{k}\right)$. Furthermore, if $\Re \Upsilon\left(h_{j}, h_{k}\right)$, then $h_{j}$ and $h_{k}$ belong to different tribes since for every solution $H$ one has that $H_{j} \leftrightarrows H_{k}$. Abusing the notation, for a tribe $t_{i}$ we denote by $\mathbb{A}\left(t_{i}\right)$ the canonical parabolic subgroup $\mathbb{A}\left(S_{i}\right)$, ie the set of letters $a_{k} \in \mathcal{A}$ so that for every $h_{j} \in t_{i}$ we have $H_{j} \leftrightarrows a_{k}$. For an item $h_{i}$ (base $\mu$, section $\sigma$ ) of $\Omega$ we set $t\left(h_{i}\right)(t(\mu)$ and $\sigma(\mu))$ to be the tribe to which $h_{i}(\mu$ or $\sigma$ ) belongs.

We say that a tribe $t_{j}$ dominates the tribe $t_{i}$ and write $t_{j} \succ t_{i}$ if and only if $\mathbb{A}\left(t_{j}\right) \geq \mathbb{A}\left(t_{i}\right)$. The relation $\succ$ is a partial order on the set of all tribes. A tribe is minimal with respect to $\succ$ if and only if it does not strictly dominate any other tribe. 
Let $\Omega=\langle\Upsilon, \Re \Upsilon\rangle$ be a generalised equation and assume that $\Re_{\Upsilon}\left(h_{i}, h_{k}\right)$. Then, for any solution $H$ of $\Omega$, we have that $H_{i} \leftrightarrows H_{k}$. If $h_{j}$ belongs to a tribe that dominates the tribe of $h_{i}$, it follows that $H_{j} \leftrightarrows H_{k}$ for all solutions $H$ of $\Omega$. Hence, every solution of $\Omega$ is a solution of $\Omega^{\prime}=\left\langle\Upsilon, \Re \Upsilon \cup\left\{\left(h_{j}, h_{k}\right)\right\}\right\rangle$.

Definition 3.2 We say that the set $\Re_{\Upsilon}$ is completed if for all $h_{j}$ that belong to a tribe that dominates the tribe of $h_{i}$, if $\Re \Upsilon\left(h_{i}, h_{k}\right)$ then $\Re \Upsilon\left(h_{j}, h_{k}\right)$. In particular, if two items $h_{i}$ and $h_{j}$ belong to the same tribe, then $\Re_{\Upsilon}\left(h_{i}, h_{k}\right)$ if and only if $\Re_{\Upsilon}\left(h_{j}, h_{k}\right)$. Moreover, if $h_{j}$ belongs to a tribe that dominates the tribe of $h_{i}$, then $\Re \Upsilon\left(h_{j}\right) \supseteq \Re_{\Upsilon}\left(h_{i}\right)$.

Remark 3.3 Without loss of generality, we further assume that for all generalised equations $\Omega=\langle\Upsilon, \Re \Upsilon\rangle$ the sets $\Re \Upsilon$ are completed.

Lemma 3.4 Let $\Omega^{\prime}=\left\langle\Upsilon^{\prime}, \Re_{\Upsilon^{\prime}}\right\rangle$ be obtained from $\Omega=\langle\Upsilon, \Re \Upsilon\rangle$ by an elementary transformation in ET1-ET5 (see [14, Section 4.2]) or a derived transformation D1, D2, D3, D5, D6 (see [14, Section 4.3]) and let $\pi$ be the corresponding epimorphism, $\pi: G_{R(\Omega)} \rightarrow G_{R\left(\Omega^{\prime}\right)}$. Let $w_{i}\left(h^{\prime}\right)=h_{i 1}^{\prime \epsilon_{i 1}} \ldots h_{i k_{i}}^{\prime \epsilon_{i k_{i}}}, \epsilon_{i j}= \pm 1, j=1, \ldots, k_{i}$, $i=1, \ldots, \rho_{\Omega}-1$, be the image of $h_{i}$ under $\pi$. Then for every $j$ the tribe $t\left(h_{i j}^{\prime}\right)$ dominates the tribe $t\left(h_{i}\right)$. Furthermore, every minimal tribe of $\Omega^{\prime}$ dominates a minimal tribe of $\Omega$.

Proof For the elementary transformations ET1,ET2 and ET3, the epimorphism $\pi$ is the identity map, $\pi\left(h_{i}\right)=\pi\left(h_{i}^{\prime}\right)$, and the statement follows.

From the description of ET5, it follows that if the number of items $\rho_{\Omega^{\prime}}$ of $\Omega^{\prime}$ equals the number of items $\rho_{\Omega}$ of $\Omega$, then $\pi\left(h_{i}\right)=h_{i}^{\prime}$ for all $i$. By definition of a solution of generalised equation, for every solution $H$ of $\Omega$, we have $H_{i} \doteq H_{i}^{\prime}$, where $\doteq$ denotes graphical equality of words and the statement follows.

Suppose now that $\rho_{\Omega^{\prime}}>\rho_{\Omega}$. Then $\pi\left(h_{i}\right)=h_{i_{j}}^{\prime}$ for all $i \neq q$ and $\pi\left(h_{q}\right)=h_{q^{\prime}-1}^{\prime} h_{q^{\prime}}^{\prime}$ (here we use the notation from the description of ET5; see [14, pages 54-55]). Since for every solution $H$ of $\Omega$ we have the graphical equality $H_{q} \doteq H_{q^{\prime}-1}^{\prime} H_{q^{\prime}}^{\prime}$, the first statement follows since, by definition, $H_{q}$ is a subword of a word in the normal form and therefore is a reduced word. Furthermore, if for some $j$ the tribe $t\left(h_{i_{j}}^{\prime}\right)$ (or $t\left(h_{q^{\prime}-1}^{\prime}\right)$ or $\left.t\left(h_{q^{\prime}}^{\prime}\right)\right)$ is minimal in $\Omega^{\prime}$, then, since equalities are graphical, it dominates the tribe $t\left(h_{i}\right)$ (or $t\left(h_{q}\right)$ ), which is also minimal.

For the transformation ET4, if $\rho_{\Omega^{\prime}}=\rho_{\Omega}$, then $\pi\left(h_{i}\right)=h_{i_{j}}^{\prime}$ for all $i$. By definition we have $H_{i} \doteq H_{i_{j}}^{\prime}$ and the statement follows in this case. We now used the notation from the description of ET4; see [14, pages 53-54]. Suppose that $\varepsilon(\mu)=\varepsilon(\Delta(\mu))$ (the proof 
is identical in the other case), then $\pi\left(h_{i}\right)=h_{\mathfrak{t}(i)}^{\prime} \cdots h_{\mathfrak{t}(i+1)-1}^{\prime}$ if $\alpha+1 \leq i \leq \beta(\mu)-1$ and $\pi\left(h_{i}\right)=h_{i_{j}}^{\prime}$ otherwise. For every solution $H$ of $\Omega$ and every $\alpha+1 \leq i \leq \beta(\mu)-1$, we have that $H_{i} \doteq H_{\mathrm{t}(i)}^{\prime} \cdots H_{\mathrm{t}(i+1)-1}^{\prime}$ and the first statement follows. Furthermore, if for some $i$ the tribe $t\left(h_{i_{j}}^{\prime}\right)$ (or one of the tribes $\left\{t\left(h_{\mathfrak{t}(i)}^{\prime}\right), \ldots, t\left(h_{\mathfrak{t}(i+1)-1}^{\prime}\right)\right\}$ ) is minimal in $\Omega^{\prime}$, then it dominates the tribe $t\left(h_{i}\right)$ (or $t\left(h_{q}\right)$ ), which is also minimal.

Since the statement is true for all elementary transformations, the statement for derived transformations now follows.

By construction, every boundary of the generalised equation in $\Omega_{i+1}$ either corresponds to a boundary of $\Omega_{i}$ or it has been introduced between $\beta\left(\mu_{i}\right)-1$ and $\rho_{\Omega_{i}}$ in $\Omega_{i}$. It follows that if the boundaries $j$ and $j+1$ of $\Omega_{i+1}$ correspond to boundaries between $k$ and $k+1$ in $\Omega_{i}$, then the tribe of an item $h_{j}$ in the generalised equation $\Omega_{i+1}$ dominates the tribe of $h_{k}$ in $\Omega_{i}$. Applying this argument recursively, we conclude that if $\Omega_{l}$, $l>i$ has been obtained from $\Omega_{i}$ by a sequence of entire transformations and the boundaries $j$ and $j+1$ of $\Omega_{l}$ correspond to boundaries between $k$ and $k+1$ in $\Omega_{i}$, then the tribe of an item $h_{j}$ in the generalised equation $\Omega_{l}$ dominates the tribe of $h_{k}$ in $\Omega_{i}$.

Let $t(\mu)$ be the tribe of a base $\mu$ in $\Omega$. Then the tribe of every item covered by $\mu$ dominates $t(\mu)$. We note that the tribe of $\mu$ in $\Omega_{i}$ is dominated by the tribe of $\mu$ in $\Omega_{i+1}$ (unless $\mu$ is completely removed when passing from $\Omega_{i}$ to $\Omega_{i+1}$ ).

\subsection{Construction of the tree $T$}

In this section we use tribes to adjust the construction of the tree $T$ which is described in [14, Chapter 4]. Note that the main result of [14, Chapter 4] remains unchanged, namely:

Proposition 3.5 [14, Proposition 4.13] For a (constrained) generalised equation $\Omega=\Omega_{v_{0}}$ over $\mathbb{F}$, one can effectively construct a locally finite, possibly infinite, oriented rooted at $v_{0}$ tree $T, T=T\left(\Omega_{v_{0}}\right)$, such that:

(1) The vertices $v_{i}$ of $T$ are labelled by generalised equations $\Omega_{v_{i}}$ over $\mathbb{F}$.

(2) The edges $v_{i} \rightarrow v_{i+1}$ of $T$ are labelled by epimorphisms

$$
\pi\left(v_{i}, v_{i+1}\right): \mathbb{G}_{R\left(\Omega_{v_{i}}\right)} \rightarrow \mathbb{G}_{R\left(\Omega_{v_{i+1}}\right)} .
$$

The edges $v_{k} \rightarrow v_{k+1}$, where $v_{k+1}$ is a leaf of $T$ and $\operatorname{tp}\left(v_{k+1}\right)=1$, are labelled by proper epimorphisms. All the other epimorphisms $\pi\left(v_{i}, v_{i+1}\right)$ are isomorphisms, in particular, edges that belong to infinite branches of the tree $T$ are labelled by isomorphisms. 
(3) Given a solution $H$ of $\Omega_{v_{0}}$, there exists a path $v_{0} \rightarrow v_{1} \rightarrow \cdots \rightarrow v_{l}$ and a solution $H^{(l)}$ of $\Omega_{v_{l}}$ such that

$$
\pi_{H}=\pi\left(v_{0}, v_{1}\right) \cdots \pi\left(v_{l-1}, v_{l}\right) \pi_{H^{(l)}} .
$$

Conversely, for every path $v_{0} \rightarrow v_{1} \rightarrow \cdots \rightarrow v_{l}$ in $T$ and every solution $H^{(l)}$ of $\Omega_{v_{l}}$ the homomorphism

$$
\pi\left(v_{0}, v_{1}\right) \cdots \pi\left(v_{l-1}, v_{l}\right) \pi_{H^{(l)}}
$$

gives rise to a solution of $\Omega_{v_{0}}$.

However our modification in the construction of the tree $T$ allows us to have an extra requirement on the type of infinite branches of the tree of Proposition 3.5. Namely, we prove the following lemma; see Lemma 3.8 below.

\section{Lemma 3.6 [14, Lemma 4.19] Let}

$$
v_{0} \rightarrow v_{1} \rightarrow \cdots \rightarrow v_{r} \rightarrow \cdots
$$

be an infinite path in the tree $T(\Omega)$. Then there exists a natural number $N$ such that all the edges $v_{n} \rightarrow v_{n+1}$ of this path with $n \geq N$ are principal edges, and one of the following conditions holds:

Linear case We have $7 \leq \operatorname{tp}\left(v_{n}\right) \leq 10$ for all $n \geq N$ and all items that do not belong to the kernel belong to a minimal tribe.

Quadratic case We have $\operatorname{tp}\left(v_{n}\right)=12$ for all $n \geq N$.

General case We have $\operatorname{tp}\left(v_{n}\right)=15$ for all $n \geq N$.

In the construction of the tree $T$ there are two kinds of edges: principal and auxiliary; see [14, page 64]. In particular, the above lemma states that one can assume that in infinite branches all edges are principal.

In order to prove the above result, we modify the linear cases [14, Cases 7-10] in the construction of the process tree $T$ as follows. Our new cases $7-10$, which we shall call cases $7^{\prime}-10^{\prime}$ will be identical to the ones described in [14], but will have an added assumption that items that do not belong to the kernel belong to a minimal tribe. In order to have this extra condition, we introduce a new case 6.5 in the construction of the process tree described in [14]. All other cases in the construction of our new process tree are verbatim to [14].

Let $\left\{t_{i}\right\}$ be the set of minimal tribes of active bases of a generalised equation $\Omega$, ie there exists an active base of $\Omega$ that belongs to the tribe $t_{i}$ and this tribe does not strictly dominate the tribe of any other active base. 
Case 6.5: There exists an item $h_{i}$ in an active section of $\Omega_{v}$ such that $\gamma_{i}=1$ In this case, for every item $h_{i}$ that does not belong to the kernel $\operatorname{Ker}\left(\Omega_{v}\right)$ of $\Omega_{v}$ and does not belong to a minimal tribe, we introduce

- a base $\lambda_{i}$, so that $\varepsilon\left(\lambda_{i}\right)=1, \alpha\left(\lambda_{i}\right)=i, \beta\left(\lambda_{i}\right)=i+1$,

- an item $h_{d_{i}}$ (along with a new boundary and re-enumerate all boundaries as appropriate) in the nonactive part of $\Omega$, and

- the dual base $\Delta\left(\lambda_{i}\right)$ of $\lambda_{i}$ so that $\varepsilon\left(\Delta\left(\lambda_{i}\right)\right)=1, \alpha\left(\Delta\left(\lambda_{i}\right)\right)=d_{i}$ and $\beta\left(\Delta\left(\lambda_{i}\right)\right)=$ $d_{i}+1$.

We set $\Re \Upsilon\left(h_{d_{i}}\right)=\Re_{\Upsilon}\left(h_{i}\right)$ in the obtained generalised equation and we call the introduced bases auxiliary.

Remark 3.7 We record the following observations.

- By construction, auxiliary bases belong to nonminimal tribes.

- Using Tietze transformations, it is immediate to check that the coordinate group associated to the obtained generalised equation $\Omega^{\prime}$ is isomorphic to the coordinate group of $\Omega$.

- If $\Omega$ satisfies the conditions of case 6.5 and $\Omega^{\prime}$ is the obtained generalised equation with auxiliary bases, using a natural one-to-one correspondence between items of $\Omega$ and $\Omega^{\prime}$, we have that $\operatorname{Ker}(\Omega) \subset \operatorname{Ker}\left(\Omega^{\prime}\right)$.

Case $7^{\prime}$ There exists an item $h_{i}$ in an active section of $\Omega_{v}$ such that $\gamma_{i}=1$, such that both boundaries $i$ and $i+1$ are closed and every item $h_{j} \notin \operatorname{Ker}\left(\Omega_{v}\right)$ belongs to a minimal tribe.

Then we remove the closed section $[i, i+1]$ together with the linear base using ET4.

Case $8^{\prime}$ There exists an item $h_{i}$ in an active section of $\Omega_{v}$ such that $\gamma_{i}=1$, one of the boundaries $i, i+1$ is open, say $i+1$, and the other is closed and every item $h_{j} \notin \operatorname{Ker}\left(\Omega_{v}\right)$ belongs to a minimal tribe.

In this case, we first perform ET5 and $\mu$-tie $i+1$ by the only base $\mu$ it intersects; then using ET1 we cut $\mu$ in $i+1$; and then we delete the closed section $[i, i+1]$ using ET4. 
Case 9' There exists an item $h_{i}$ in an active section of $\Omega_{v}$ such that $\gamma_{i}=1$ and such that both boundaries $i$ and $i+1$ are open and every item $h_{j} \notin \operatorname{Ker}\left(\Omega_{v}\right)$ belongs to a minimal tribe. In addition, there is a closed section $\sigma$ such that $\sigma$ contains exactly two bases $\mu_{1}$ and $\mu_{2}, \sigma=\sigma\left(\mu_{1}\right)=\sigma\left(\mu_{2}\right)$ and $\mu_{1}, \mu_{2}$ is not a pair of matched bases, ie $\mu_{1} \neq \Delta\left(\mu_{2}\right)$; moreover, in the generalised equation $\widetilde{\Omega}_{v}=\mathrm{D} 3(\Omega)$ all the bases obtained from $\mu_{1}, \mu_{2}$ by ET1 when constructing $\widetilde{\Omega}_{v}$ from $\Omega_{v}$, do not belong to the kernel of $\widetilde{\Omega}_{v}$.

In this case, using ET5, we $\mu_{1}$-tie all the boundaries that intersect $\mu_{1}$; using ET2, we transfer $\mu_{2}$ onto $\Delta\left(\mu_{1}\right)$; and remove $\mu_{1}$ together with the closed section $\sigma$ using ET4.

Case 10' There exists an item $h_{i}$ in an active section of $\Omega_{v}$ such that $\gamma_{i}=1$ and such that both boundaries $i$ and $i+1$ are open and every item $h_{j} \notin \operatorname{Ker}\left(\Omega_{v}\right)$ belongs to a minimal tribe.

In this event we close the section $[i, i+1]$ using D1 and remove it using ET4.

A crucial notion for the analysis of the process tree is that of a complexity of generalised equation. By convention, the auxiliary bases that we introduced in Case 6.5 do not contribute to the complexity of a generalised equation. Formally, for a closed section $\sigma \in \Sigma(\Omega)$ denote by $n(\sigma)$ the number of nonauxiliary bases in $\sigma$. The complexity of a generalised equation $\Omega$ is defined as

$$
\operatorname{comp}=\operatorname{comp}(\Omega)=\sum_{\sigma \in A \Sigma(\Omega)} \max \{0, n(\sigma)-2\}
$$

Note that the complexity of a generalised equation defined above differs slightly from the definition used in [14] since it does not count auxiliary bases. Uncontrolled addition of auxiliary bases could potentially result in a new source of infinite branches in the construction of the tree. The hierarchy on tribes is used to control this phenomena: newly added auxiliary bases go up in the hierarchy and when they are maximal, no new auxiliary bases are further introduced. At this point, the complexity of a generalised equation defined in [14] does not increase and we can analyse the corresponding generalised equation using the process from [14].

Given a generalised equation $\Omega$, we construct an oriented rooted tree $T^{\prime}(\Omega)$ as follows: we start from the root $v_{0}$ to which we assigned $\Omega$ and proceed by induction on the height of the tree. Suppose, by induction, that the tree $T^{\prime}(\Omega)$ is constructed up to height $n$, and let $v$ be a vertex of height $n$. We now describe how to extend the tree from $v$. The construction of the outgoing edges from $v$ depends on which of the 15 cases takes place at the vertex $v$. We always assume that if the generalised equation $\Omega_{v}$ satisfies the assumptions of case $i$, then $\Omega_{v}$ does not satisfy the assumptions of all the cases $j$ with $j<i$. 
Using this procedure we obtain a tree $T^{\prime}=T^{\prime}(\Omega)$ satisfying all the properties listed in Proposition 3.5, this is our new process tree. We are now in the position to describe infinite branches of the tree $T^{\prime}$.

\section{Lemma 3.8 [14, Lemma 4.19] Let}

$$
v_{0} \rightarrow v_{1} \rightarrow \cdots \rightarrow v_{r} \rightarrow \cdots
$$

be an infinite path in the tree $T^{\prime}(\Omega)$. Then there exists a natural number $N$ such that all the edges $v_{n} \rightarrow v_{n+1}$ of this path with $n \geq N$ are principal edges, and one of the following conditions holds:

Linear case We have $7^{\prime} \leq \operatorname{tp}\left(v_{n}\right) \leq 10^{\prime}$ for all $n \geq N$.

Quadratic case We have $\operatorname{tp}\left(v_{n}\right)=12$ for all $n \geq N$.

General case We have $\operatorname{tp}\left(v_{n}\right)=15$ for all $n \geq N$.

Proof Observe that if in an infinite branch the type of a vertex is 6 , then the pair of matched bases cannot be auxiliary since the dual of an auxiliary base is by construction nonactive. Hence after applying case 6 the complexity of a generalised equation strictly decreases.

The argument given in the proof of [14, Lemma 4.19] shows that infinite branches are of type 12,15 or $6.5,7^{\prime}-10^{\prime}$.

We are left to show that in an infinite branch of type $6.5,7^{\prime}-10^{\prime}$, the case 6.5 can only occur finitely many times. By Lemma 3.4, without loss of generality we may assume that the minimal tribe (of an active base) is constant throughout the infinite branch.

Recall that an item does not belong to the kernel of a generalised equation if after an elimination process the item is uncovered (see the description of derived transformation D4 in [14, pages 57-58]). If in an infinite branch the type of $\Omega_{v_{i}}$ is 6.5 , then either the type of $\Omega_{v_{i+1}}$ or of $\Omega_{v_{i+2}}$ is $7^{\prime}-10^{\prime}$. Indeed, assume that the type of $\Omega_{v_{i+1}}$ is also 6.5, then each item which does not belong to the kernels of $\Omega_{v_{i}}$ and of $\Omega_{v_{i+1}}$ and which does not belong to a minimal tribe is covered by two auxiliary bases. Therefore, in the elimination process for $\Omega_{v_{i+2}}$ every such item is covered at least twice and so it belongs to $\operatorname{Ker}\left(\Omega_{v_{i+2}}\right)$. Hence the type of $v_{i+2} \geq 7^{\prime}$ and all items in $\Omega_{v_{i+2}}$ that do not belong to $\operatorname{Ker} \Omega_{v_{i+2}}$ belong to the minimal tribe. Furthermore, it follows from [14, Lemma 7.10] that in an infinite branch, cases $7^{\prime}-10^{\prime}$ leave the kernel of the generalised equation invariant and so the type of $\Omega_{v_{i+n}}$ is never 6.5 for $n \geq 2$. We conclude that the infinite branch is of type $7^{\prime}-10^{\prime}$ as desired. 


\subsection{Construction of the finite tribal tree $T_{0}$}

In [14, Chapter 7], we proved that one can associate specific groups of automorphisms to $\Omega$ and determine a finite subtree $T_{0}(\Omega)$ of the infinite tree $T(\Omega)$ such that for any solution of $\Omega$, there exists another solution, which is equivalent to the original one modulo associated automorphisms and which factors through the finite tree $T_{0}(\Omega)$. Furthermore, leaves of the tree $T_{0}(\Omega)$ are either of type 1 , ie correspond to proper quotients, or they only contain nonactive sections with periodic structures; see [14, Proposition 7.1].

The aim of this section is to present a construction of an analogous finite tree that, on the one hand, takes into account the modifications we made in the previous section (Cases $6.5,7^{\prime}-10^{\prime}$ ) in the construction of the process tree $T^{\prime}(\Omega)$ and, on the other hand, allows for a subtle analysis of the behaviour of tribes in infinite branches.

More precisely, in this section we introduce the notions of tribal generalised equations of types 12, 15 and linear type (see Definitions 3.24, 3.26 and 3.28) and prove:

Proposition 3.29 For a (constrained) generalised equation $\Omega=\Omega_{v_{0}}$, one can effectively construct a finite oriented rooted at $v_{0}$ tree $T_{0}, T_{0}=T_{0}\left(\Omega_{v_{0}}\right)$ such that:

(1) The tree $T_{0}$ is a subtree of the tree $T^{\prime}(\Omega)$.

(2) To the root $v_{0}$ of $T_{0}$ we assign a recursive group of automorphisms $\operatorname{Aut}(\Omega)$ related to tribal generalised equations of type 12,15 and linear in the tree $T_{0}$.

(3) For any solution $H$ of a generalised equation $\Omega$ there exists a leaf $w$ of the tree $T_{0}(\Omega), \operatorname{tp}(w)=1,2$, and a solution $H^{[w]}$ of the generalised equation $\Omega_{w}$ such that

- $H^{[w]}<\operatorname{Aut}(\Omega) H$;

- if $\operatorname{tp}(w)=2$ and the generalised equation $\Omega_{w}$ contains nonconstant nonactive sections, then either

- there exists a period $P$ such that $H^{[w]}$ is periodic with respect to the period $P$ and the generalised equation $\Omega_{w}$ is either singular of strongly singular with respect to the periodic structure $\mathcal{P}\left(H^{[w]}, P\right)$ or

- the section has been transferred to the nonactive part by an auxiliary base.

3.3.1 Quadratic case The goal of this section is to prove a refinement of the following lemma from [14] and analyse the behaviour of tribes in an infinite branch of type 12 . 
Lemma 3.9 [14, Lemma 7.11] Let $v_{0} \rightarrow v_{1} \rightarrow \cdots \rightarrow v_{n} \rightarrow \cdots$ be an infinite path in the tree $T^{\prime}(\Omega)$, where $\operatorname{tp}\left(v_{i}\right)=12$ for all $i$, and $\Omega_{v_{0}}, \Omega_{v_{1}}, \ldots, \Omega_{v_{n}}, \ldots$ be the sequence of corresponding generalised equations. Then among $\left\{\Omega_{v_{i}}\right\}$ some generalised equation occurs infinitely many times. Furthermore, if $\Omega_{v_{k}}=\Omega_{v_{l}}$, then $\pi\left(v_{k}, v_{l}\right)$ is a $\mathbb{G}$-automorphism of the coordinate group $\mathbb{G}_{R\left(\Omega_{v_{k}}\right)}$ invariant with respect to the nonquadratic part.

More precisely, we aim to show that all the items of the quadratic section that are not fixed by the group of automorphisms assigned to the vertex belong to the same minimal tribe and so, in particular, all of them commute with the same set of items. Furthermore, items of the quadratic section that are fixed by the automorphisms belong to tribes that dominate the minimal tribe (the one defined by any of the items which is not fixed by the automorphisms).

Let us begin with the analysis of an infinite branch of type 12 . Let $\Omega=\langle\Upsilon, \Re \Upsilon\rangle$ be a quadratic constrained generalised equation of type 12 . Throughout this section we will be mostly concerned with active bases, boundaries, items etc, ie bases, items and boundaries of the (active) quadratic part. When no confusion arises, we will omit saying that the bases (items, boundaries etc) are active.

Let us consider an infinite branch of type 12 in the tree $T(\Omega)$,

$$
\Omega_{0} \rightarrow \Omega_{1} \rightarrow \cdots
$$

Then, by [14, Lemma 7.12], there exists a generalised equation $\Omega_{r_{0}}$ that repeats infinitely many times in the infinite branch: $\Omega_{r_{0}}=\Omega_{r_{1}}=\cdots$. Without loss of generality, we assume that $r_{0}=0$. Furthermore, the canonical epimorphism $\pi\left(r_{i}, r_{j}\right)$ from $G_{\Omega_{r_{i}}}$ to $G_{\Omega_{r_{j}}}$ is an automorphism of $G_{\Omega_{r_{i}}}$. In fact, since there are finitely many different generalised equations appearing in the infinite branch, without loss of generality, we may assume that every generalised equation of the infinite branch appears in it infinitely many times.

Since the branch is infinite and $\Omega_{0}$ repeats infinitely many times, we have that the complexity, the number of active (nonauxiliary) bases and the number of active items is constant throughout the branch, ie $\operatorname{comp}\left(\Omega_{i}\right)=\operatorname{comp}\left(\Omega_{i+1}\right), n_{A}\left(\Omega_{i}\right)=n_{A}\left(\Omega_{i+1}\right)$ and $\rho_{\Omega_{i}}=\rho_{\Omega_{i+1}}$ for all $i$. Furthermore, if $\mu_{i}$ is the carrier of $\Omega_{i}$, then every $\mu_{i}$-tying introduces a new boundary. There is a natural one-to-one correspondence between the bases of $\Omega_{i}$ and $\Omega_{i+1}$. Namely, the base $\mu_{i}$ in $\Omega_{i+1}$ is the only leading base which is not the carrier and all the other bases are naturally preserved. Hence, we may assume that the sets of bases of $\Omega_{i}$ and $\Omega_{i+1}$ coincide. 
Lemma 3.10 Let $\Omega$ be a generalised equation of type 12 that repeats infinitely many times in the infinite branch. Then one can declare the active part of the generalised equation $\Omega$ to contain only one closed section.

Proof Suppose that $\Omega$ contains more than one (active) closed section: $[1, i]$ and $[i, j]$. For every $n$ we partition the set of bases $\mathcal{B S}\left(\Omega_{n}\right)$ of $\Omega_{n}$ into two. Let $L\left(\Omega_{n}\right)$ be the set of bases $\lambda$ of $\Omega_{n}$ so that $\alpha(\lambda)$ and $\beta(\lambda)$ lie to the left of the boundary $i$ of $\Omega$. Set $R\left(\Omega_{n}\right)$ to be the set of bases $\lambda$ of $\Omega_{n}$ so that $\alpha(\lambda)$ and $\beta(\lambda)$ lie to the right of the boundary $i$ of $\Omega$. Since the boundary $i$ is closed in $\Omega$, it follows that $\mathcal{B S}\left(\Omega_{n}\right)=L \sqcup R$.

Notice that if $\lambda \in R\left(\Omega_{n}\right)$, then $\lambda \in R\left(\Omega_{n+1}\right)$.

We first observe that the carrier base of $\Omega_{n}$ belongs to $L\left(\Omega_{n}\right)$ for all $n$. Indeed, if $\Omega_{k+1}$ is the generalised equation so that its carrier $\mu_{k+1}$ belongs to $R\left(\Omega_{k+1}\right)$ and for all $n \leq k$ the carrier $\mu_{n}$ of $\Omega_{n}$ belongs to $L\left(\Omega_{n}\right)$, then it follows that $n_{A}\left(\Omega_{k}\right)>$ $n_{A}\left(\Omega_{k+1}\right)$, a contradiction.

We now show that if $v \in R(\Omega)$ and $\Delta(v) \in L(\Omega)$, then $\Delta(v)$ is never carrier. Assume the contrary, ie $\Delta(v)=\mu_{l}$ is the carrier of $\Omega_{l}$ for some $l$. Let $\lambda$ be a transfer base. In the infinite branch, the base $\lambda$ is transferred infinitely many times. Let $\Omega_{m}$ be the next time $\lambda$ is transferred. It is clear that the carrier $\mu_{m}$ belongs to $R\left(\Omega_{m}\right)$, deriving a contradiction with the above observation.

Since, the generalised equation $\Omega$ repeats, it follows that no boundaries are introduced between $\alpha(\Delta(v))$ and $\beta(\Delta(v))$. Therefore, one can declare the section $[i, j]$ of $\Omega$ to be nonactive.

Let $\lambda$ be a base of $\Omega$. We call the number $l(\lambda)=\beta(\lambda)-\alpha(\lambda)$ the length of $\lambda$ (in $\Omega$ ). We call the base short if $l(\lambda)=1$ and long otherwise.

Let $\lambda_{1}$ and $\lambda_{2}$ be two long bases of $\Omega$. Notice that since there are no free boundaries in $\Omega$ and since, by Lemma 3.10, the generalised equation $\Omega$ has only one active closed section, then every boundary touches precisely two bases. Therefore, the bases $\lambda_{1}$ and $\lambda_{2}$ either do not overlap or if they do, then $\alpha\left(\lambda_{2}\right)=\beta\left(\lambda_{1}\right)-1$ or $\alpha\left(\lambda_{1}\right)=\beta\left(\lambda_{2}\right)-1$.

For every generalised equation $\Omega_{r}$ from the infinite branch and any $h_{i}^{(r)} \in \Omega_{r}$, the set of words $\left\{\pi\left(v_{r}, v_{s}\right)\left(h_{i}^{(r)}\right) \mid s>r, \Omega_{r}=\Omega_{s}\right\}$ is either finite or infinite, ie the orbit of an item under the automorphisms associated to $\Omega_{r}$ is either a finite or an infinite set. Define the set $\mathcal{F}\left(\Omega_{r}\right)$ to be the set of items of $\Omega_{r}$ for which this set is finite and let $\mathcal{H}\left(\Omega_{r}\right)$ be its complement. 
Notice that by the description of automorphisms associated to a generalised equation of type 12 (see [14, Lemma 7.7]) any item that belongs to a quadratic-coefficient base of $\Omega_{r}$ or does not belong to the quadratic part is fixed by the automorphisms, and so belongs to the set $\mathcal{F}\left(\Omega_{r}\right)$.

Let $h_{i}^{(0)} \in \mathcal{F}\left(\Omega_{0}\right)$ and let

$$
\left\{\varphi_{s}\left(h_{i}^{(0)}\right) \mid \varphi_{s}=\pi\left(v_{0}, v_{s}\right)\left(h_{i}^{(0)}\right), \Omega_{0}=\Omega_{s}\right\}=\left\{w_{i 1}, \ldots, w_{i k}\right\}
$$

be the orbit of $h_{i}^{(0)}$ by the family of automorphisms $\left\{\varphi_{s}\right\}$ associated to the vertex $v_{0}$. Without loss of generality we can assume in an infinite branch the sets $I_{l}=\{s \in \mathbb{N} \mid$ $\left.\varphi_{s}=\pi\left(v_{0}, v_{s}\right)\left(h_{i}^{(0)}\right)=w_{i k}\right\}, l=1, \ldots, k$ are all infinite.

Remark 3.11 By replacing the infinite branch at $\Omega_{0}$ by $k$ identical branches at $\Omega_{0, l}$, $l=1, \ldots, k$ and assigning to $\Omega_{0, l}$ the group of automorphisms $\left\{\varphi_{s} \mid s \in I_{l}\right\}$, we may assume that if $h_{i}^{(0, l)} \in \mathcal{F}\left(\Omega_{0, l}\right)$, then

$$
\varphi_{s}\left(h_{i}^{(0, l)}\right)=w_{i, l} \quad \text { for all } s \in I_{l}, l=1, \ldots, k .
$$

Let $\Omega_{s}=\Omega_{0}$ be so that for all $h_{i}^{(0)} \in \mathcal{F}(\Omega)$ we have that $\pi\left(v_{0}, v_{s}\right)\left(h_{i}^{(0)}\right)=w\left(h^{(s)}\right)=$ $h_{i_{1}}^{(s)} \cdots h_{i_{n}}^{(s)}$. For all $s^{\prime}>s$ such that $\Omega_{s^{\prime}}=\Omega_{0}$, we have that $\pi\left(v_{0}, v_{s^{\prime}}\right)\left(h_{i}^{(0)}\right)=\pi\left(v_{s}, v_{s^{\prime}}\right)\left(\pi\left(v_{0}, v_{s}\right)\left(h_{i}^{(0)}\right)\right)=\pi\left(v_{s}, v_{s^{\prime}}\right)\left(h_{i_{1}}^{(s)} \cdots h_{i_{n}}^{(s)}\right)=h_{i_{1}}^{\left(s^{\prime}\right)} \cdots h_{i_{n}}^{\left(s^{\prime}\right)}$.

From the definition of $\pi\left(v_{s}, v_{s^{\prime}}\right)$, we conclude that $\pi\left(v_{s}, v_{s^{\prime}}\right)\left(h_{i_{j}}^{(s)}\right)=h_{i_{j}}^{\left(s^{\prime}\right)}$ and so $h_{i_{j}}^{(s)} \in \mathcal{F}\left(\Omega_{s}\right)$ and $\pi\left(v_{s}, v_{t}\right)\left(h_{i_{j}}^{(s)}\right)=h_{m}^{(t)}$, for all $s<t<s^{\prime}, \Omega_{s}=\Omega_{s^{\prime}}$.

Remark 3.12 Without loss of generality we may assume that $h_{i}^{(0)} \in \mathcal{F}\left(\Omega_{0}\right)$ if and only if $\varphi_{s}\left(h_{i}^{(0)}\right)=h_{i}^{(0)}$ for all automorphism associated to $\Omega_{0}$.

Furthermore, we have that if $h_{i}^{(0)} \in \mathcal{F}\left(\Omega_{0}\right)$ then $\pi\left(v_{0}, v_{t}\right)\left(h_{i}^{(0)}\right)=h_{m}^{(t)} \in \mathcal{F}\left(\Omega_{t}\right)$ for all $t>0$. Hence $\left|\mathcal{F}\left(\Omega_{0}\right)\right| \leq \cdots \leq\left|\mathcal{F}\left(\Omega_{t}\right)\right| \leq \cdots$. Then, since the number $\left|\mathcal{F}\left(\Omega_{0}\right)\right|$ is bounded above by $\rho_{\Omega_{0}}=\rho_{\Omega_{t}}$, without loss of generality, we may assume that the number $\left|\mathcal{F}\left(\Omega_{0}\right)\right|$ is constant throughout the branch, ie $\left|\mathcal{F}\left(\Omega_{0}\right)\right|=\left|\mathcal{F}\left(\Omega_{t}\right)\right|$ for all $t$.

Given a generalised equation $\Omega$, we define $\mathfrak{m}(\Omega)$ to be the set of minimal tribes of the generalised equation:

$\mathfrak{m}(\Omega)=\left\{t\left(h_{j}\right) \mid h_{j} \in \mathcal{H}(\Omega)\right.$ and $t\left(h_{j}\right)$ does not strictly dominate $t\left(h_{k}\right)$ for all $\left.h_{k} \in \mathcal{H}(\Omega)\right\}$ and $h\left(\mathfrak{M}_{i}(\Omega)\right)$ to be the set of items that belong to a given minimal tribe $\mathfrak{M}_{i}$ of $\Omega$ : $\left.h\left(\mathfrak{M}_{i}(\Omega)\right)=\left\{h_{j} \in \mathcal{H}(\Omega)\right\} \mid t\left(h_{j}\right) \in \mathfrak{M}_{i}, \mathfrak{M}_{i} \in \mathfrak{m}(\Omega)\right\}$. 
We draw the reader's attention to the fact that the minimal tribes are defined using the items from $\mathcal{H}(\Omega)$, which, by definition, are active.

Lemma 3.13 In an infinite branch of type 12 , there exists $i \in \mathbb{N}$ so that $\mathfrak{m}\left(\Omega_{i}\right)=$ $\mathfrak{m}\left(\Omega_{j}\right)$ for all $j>i$. Furthermore, the cardinality of the set $h\left(\mathfrak{M}_{k}\left(\Omega_{i}\right)\right)$ is constant, ie $\left|h\left(\mathfrak{M}_{k}\left(\Omega_{i}\right)\right)\right|=\left|h\left(\mathfrak{M}_{k}\left(\Omega_{j}\right)\right)\right|$ for every minimal tribe $\mathfrak{M}_{k} \in \mathfrak{m}\left(\Omega_{i}\right)=\mathfrak{m}\left(\Omega_{j}\right)$.

Proof Since the generalised equation repeats infinitely many times, $\Omega_{r_{0}}=\Omega_{r_{1}}=\cdots$ and since there are finitely many different tribes, for sufficiently large $i$ and passing to a subsequence $\Omega_{r_{i_{0}}}=\Omega_{r_{i_{1}}}=\cdots$, we may assume that the tribe of every item $h_{k}^{\left(r_{i_{j}}\right)}$ is the same, ie $t\left(h_{k}^{\left(r_{i_{0}}\right)}\right)=t\left(h_{k}^{\left(r_{i_{j}}\right)}\right)$ for all $j \in \mathbb{N}$. Hence $\mathfrak{m}\left(\Omega_{r_{i_{0}}}\right)=\mathfrak{m}\left(\Omega_{r_{i_{j}}}\right)$ and $\left|h\left(\mathfrak{M}_{k}\left(\Omega_{r_{i_{0}}}\right)\right)\right|=\left|h\left(\mathfrak{M}_{k}\left(\Omega_{r_{i_{j}}}\right)\right)\right|$ for all $\mathfrak{M}_{k} \in \mathfrak{m}\left(\Omega_{r_{i_{0}}}\right)=\mathfrak{m}\left(\Omega_{r_{i_{j}}}\right)$. To simplify the notation, set $i_{0}=0$.

It follows from the above equalities that in order to prove that $\mathfrak{m}\left(\Omega_{i}\right)=\mathfrak{m}\left(\Omega_{j}\right)$ and that $\left|h\left(\mathfrak{M}_{k}\left(\Omega_{i}\right)\right)\right|=\left|h\left(\mathfrak{M}_{k}\left(\Omega_{j}\right)\right)\right|$ for all $i, j \in \mathbb{N}$ and $\mathfrak{M}_{k} \in \mathfrak{m}\left(\Omega_{i}\right)$, it suffices to show that $\mathfrak{m}\left(\Omega_{i}\right) \supseteq \mathfrak{m}\left(\Omega_{i+1}\right)$ and that $\left|h\left(\mathfrak{M}_{k}\left(\Omega_{i}\right)\right)\right| \geq\left|h\left(\mathfrak{M}_{k}\left(\Omega_{i+1}\right)\right)\right|$.

Let $\mu$ be the carrier of $\Omega_{i}$, let $j, j+1$ be the boundaries that touch a transfer base (that is $j+1 \leq \beta(\mu)-1)$ and let $(j, \mu, \mathfrak{b}(j))$ be the corresponding boundary connection introduced in the entire transformation. Suppose $\mathfrak{b}(j)$ is introduced between $\mathfrak{l}(\mathfrak{b}(j))$ and $\mathfrak{l}(\mathfrak{b}(j))+1$. By Lemma 3.4, it follows that $t\left(h_{\mathfrak{r}(\mathfrak{b}(j))}^{(i+1)}\right)$ dominates $t\left(h_{j}^{(i)}\right)$ and $t\left(h_{\mathfrak{b}(j)}^{(i+1)}\right)$ dominates $t\left(h_{\mathfrak{l}(\mathfrak{b}(j))}^{(i)}\right)$. Therefore, we have that $\mathfrak{m}\left(\Omega_{i}\right) \supseteq \mathfrak{m}\left(\Omega_{i+1}\right)$ and $\left|h\left(\mathfrak{M}_{k}\left(\Omega_{i}\right)\right)\right| \geq\left|h\left(\mathfrak{M}_{k}\left(\Omega_{i+1}\right)\right)\right|$ for all minimal tribes $\mathfrak{M}_{k}$.

We call the items of $\Omega_{i}$ that belong to a minimal tribe minimal.

Recall that the carrier base $\mu_{i}$ of $\Omega_{i}$ is a long base. Since there are no free boundaries, there are exactly $\beta\left(\mu_{i}\right)-1$ transfer bases and all of them are short. Moreover, $\mu_{i}$ is a short transfer base of $\Omega_{i+1}$; see Figure 1 .

Definition 3.14 Let $\mu$ be the carrier base of $\Omega$. We take the (uniquely defined) transfer base $\lambda$ so that $\alpha(\lambda)=1$ and we $\mu$-tie the boundary $2=\beta(\lambda)$. We then transfer $\lambda$ from $\mu$ onto $\Delta(\mu)$. Now we cut $\mu$ in the boundary 2 and delete the section [1,2].

It follows from the definition that the (usual) entire transformation D5 is a composition of the transformations just introduced (namely of $\beta(\mu)-2$ such transformations). From now on, we abuse the terminology and refer to the introduced transformation as to the entire transformation and refer to the usual entire transformation as D5 or complete entire transformation. 
Lemma 3.15 In an infinite branch $\Omega_{0} \rightarrow \Omega_{1} \rightarrow \cdots$ of type 12 , there exists a generalised equation $\Omega_{p}$ such that

(A) either the item $h_{1}^{(p)}$ belongs to a minimal tribe of $\Omega_{p}$, or

(B) the entire transformation introduces a new boundary in an item that belongs to a minimal tribe of $\Omega_{p}$.

Proof Let $h_{j}^{\left(r_{0}\right)}$ be a minimal item of $\Omega_{r_{0}}$. Since $h_{j}^{\left(r_{0}\right)} \in \mathcal{H}\left(\Omega_{r_{0}}\right)$, by definition of the set $\mathcal{H}\left(\Omega_{r_{0}}\right)$, the orbit of $h_{j}^{\left(r_{0}\right)}$ under the automorphisms associated to $\Omega_{r_{0}}$ is infinite. Therefore, there exists $p \in \mathbb{N}$ so that

$$
\left|\pi\left(r_{0}, i\right)\left(h_{j}^{\left(r_{0}\right)}\right)\right|=1 \text { for all } i \leq p \text { and }\left|\pi\left(r_{0}, p+1\right)\left(h_{j}^{\left(r_{0}\right)}\right)\right| \geq 2 .
$$

Hence either

- a base covering the item $\pi\left(r_{0}, p\right)\left(h_{j}^{\left(r_{0}\right)}\right)=h_{j_{p}}^{(p)}$ is transferred (case (A) of the lemma) and so, by Remark 3.12, one can assume that $j_{p}=1$, or

- in the entire transformation of the generalised equation $\Omega_{p}$, a new boundary is introduced between the boundaries $j_{p}$ and $j_{p}+1$ (case (B) of the lemma).

Furthermore, since $\pi\left(r_{0}, i\right)\left(h_{j}^{\left(r_{0}\right)}\right)=h_{j_{i}}^{(i)}$ for all $i \leq p$, it follows that the tribe of $h_{j_{i}}^{(i)}$ is the same for all $i \leq p$, ie $t\left(h_{j_{i}}^{(i)}\right)=t\left(h_{j}^{\left(r_{0}\right)}\right)$. Hence if $h_{j}^{\left(r_{0}\right)}$ belongs to a minimal tribe $\mathfrak{M}_{k}$ in $\Omega_{0}$, then $h_{j_{i}}^{(i)}$ also belongs to the tribe $\mathfrak{M}_{k}$ and, by Lemma $3.13, \mathfrak{M}_{k}$ is a minimal tribe in $\Omega_{p}$.

Lemma 3.16 In an infinite branch of type 12,

$$
\Omega_{0} \rightarrow \Omega_{1} \rightarrow \cdots,
$$

where $\Omega_{i}$ is obtained from $\Omega_{i+1}$ by an entire transformation, there exists a generalised equation $\Omega_{k}$ of the infinite branch such that the item $h_{1}^{(k)}$ is minimal (case (A) of Lemma 3.15 holds).

Proof By Lemma 3.15, it suffices to show that if in the infinite branch there exists a generalised equation that satisfies case (B) of Lemma 3.15, then there exists a generalised equation that satisfies case (A).

Without loss of generality, we may assume that the generalised equation $\Omega_{0}$ satisfies case (B) of Lemma 3.15, ie the entire transformation introduces a boundary connection $(2, \mu, \mathfrak{b}(2))$ and the boundary $\mathfrak{b}(2)$ is between the boundaries $\mathfrak{l}(\mathfrak{b}(2))$ and $\mathfrak{l}(\mathfrak{b}(2))+1$, where $\mu$ is the carrier base of $\Omega_{0}$ and $h_{\mathfrak{l}(\mathfrak{b}(2))}^{(0)}$ is a minimal item that belongs to some minimal tribe $\mathfrak{M}$. 
Since, by Lemma 3.13, the number of minimal items of a fixed minimal tribe $\mathfrak{M}$ in the infinite branch is constant, it follows that either $h_{\mathfrak{l}(\mathfrak{b}(2))}^{(1)}$ or $h_{\mathfrak{b}(2)}^{(1)}$ is minimal and belongs to $\mathfrak{M}$. If $h_{\mathfrak{b}(2)}^{(1)} \in h(\mathfrak{M})$, then, by Lemma 3.4, we have that $h_{1}^{(0)} \in h(\mathfrak{M})$ and hence $\Omega_{0}$ satisfies case (A) of Lemma 3.15.

Assume now that $h_{\mathfrak{l}(\mathfrak{b}(2))}^{(1)}$ is minimal and belongs to $\mathfrak{M}$. Let $k$ be so that for all the boundary connections $(i, \mu, \mathfrak{b}(i)), i=2, \ldots, k-1$, one has $\mathfrak{l}(\mathfrak{b}(i))=\mathfrak{l}(\mathfrak{b}(2))$ and $\mathfrak{l}(\mathfrak{b}(k))>\mathfrak{l}(\mathfrak{b}(2))$.

Suppose that $\Omega_{i}, i=1, \ldots, k-1$, does not satisfy case (A) of Lemma 3.15. Then, since the number of minimal items of the minimal tribe $\mathfrak{M}$ is constant in the infinite branch, it follows that $h_{\mathfrak{b}(k-1)}^{(k)}$ is minimal and belongs to $h(\mathfrak{M})$ (note that here $k-1$ is the boundary of $\left.\Omega_{0}\right)$. By definition of $k$ and Lemma 3.4, the tribe of $h_{\mathfrak{b}(k-1)}^{(k)}$ dominates the tribe of $h_{k-1}^{(0)}$ and hence, $h_{k-1}^{(0)}$ is minimal and belongs to $h(\mathfrak{M})$. We conclude that the generalised equation $\Omega_{k}$ falls under case (A) of Lemma 3.15.

Corollary 3.17 Let $v_{0} \rightarrow v_{1} \rightarrow \cdots \rightarrow v_{n} \rightarrow \cdots$ be an infinite path in the tree $T(\Omega)$, where $\operatorname{tp}\left(v_{i}\right)=12$ for all $i$, and $\Omega_{v_{0}}, \Omega_{v_{1}}, \ldots, \Omega_{v_{n}}, \ldots$ be the sequence of corresponding generalised equations. Then among $\left\{\Omega_{v_{i}}\right\}$ some generalised equation $\Omega_{i_{k}}$ occurs infinitely many times and $h_{1}^{\left(i_{k}\right)}$ is minimal.

Lemma 3.18 Let $\Omega_{0}$ be a generalised equation of type 12 that repeats infinitely many times in the infinite branch. Let $\mu$ be the carrier of $\Omega_{0}$ and let $h_{1}^{(0)}$ be an item from a minimal tribe $\mathfrak{M}$. Then the item $h_{\beta(\mu)-1}^{(0)}$ is also minimal and belongs to $h(\mathfrak{M})$. In particular, the item $h_{1}^{(1)}$ of $\Omega_{1}$, where $\Omega_{1}$ is obtained from $\Omega_{0}$ by a complete entire transformation is minimal and belongs to $h(\mathfrak{M})$.

The tribe of every item covered by $\mu$ dominates the minimal tribe $\mathfrak{M}$ of $h_{1}^{(0)}$. Furthermore, if an item $h_{i}^{(0)}$ covered by $\mu$ strictly dominates the minimal tribe, then $\pi\left(v_{0}, v_{1}\right)\left(h_{i}^{(0)}\right)=h_{j}^{(1)}$ and, in particular, $h_{i}^{(0)} \in \mathcal{F}\left(\Omega_{0}\right)$.

Proof Recall that every $\mu$-tying introduces a new boundary. We only consider the case when $\varepsilon(\Delta(\mu))=\varepsilon(\mu)=1$. The remaining cases are analogous. We $\mu$-tie all the boundaries $2, \ldots, \beta(\mu)-1$ thus introducing boundaries $n_{2}, \ldots, n_{\beta(\mu)-1}$ between the boundaries $\mathfrak{l}\left(n_{2}\right)$ and $\mathfrak{l}\left(n_{2}\right)+1, \ldots, \mathfrak{l}\left(n_{\beta(\mu)-1}\right)$ and $\mathfrak{l}\left(n_{\beta(\mu)-1}\right)+1$. We will denote the corresponding items of $\Omega_{1}$ by

$$
h_{\mathfrak{l}(j)}^{(1)}, \quad h_{\mathfrak{l}(j)+1}^{(1)} \quad \text { and } \quad h_{n_{j}}^{(1)} .
$$

Since the number of minimal items of a fixed minimal tribe in the infinite branch is constant, it follows that at least one of the items $h_{\mathfrak{l}(2)}^{(1)}$ and $h_{n_{2}}^{(1)}$ belongs to $h(\mathfrak{M})$. 
Hence, the item $h_{\mathfrak{l}(2)}^{(0)}$ of $\Omega_{0}$ is minimal and belongs to $h(\mathfrak{M})$. Therefore, since the number $|h(\mathfrak{M})|$ is constant both $h_{\mathfrak{l}(2)}^{(1)}$ and $h_{n_{2}}^{(1)}$ are minimal and belong to $h(\mathfrak{M})$.

If $\mathfrak{l}(3)>\mathfrak{l}(2)$, we conclude that the item $h_{2}^{(0)}$ of $\Omega_{0}$ is minimal and belongs to $h(\mathfrak{M})$. Either $\mathfrak{l}(2)=\mathfrak{l}(3)$ or, otherwise, we can repeat the above argument for $h_{2}$. Let $m$ be maximal so that $\mathfrak{l}(2)=\mathfrak{l}(m)$. Then the items $h_{n_{i}}^{(1)}$ of $\Omega_{1}$ belong to the same tribe as the item $h_{i}^{(0)}$ of $\Omega_{0}, i=2, \ldots, m-1$, and since the number $|h(\mathfrak{M})|$ in the infinite branch is constant, then $h_{n_{m}}^{(1)}$ is minimal and belongs to $h(\mathfrak{M})$. It follows that the item $h_{m}^{(0)}$ is minimal and belongs to $h(\mathfrak{M})$, hence we can repeat the argument for $h_{m}^{(0)}$.

We conclude that the item $h_{\beta(\mu)-1}^{(0)}$ of $\Omega_{0}$ is minimal and belongs to $h(\mathfrak{M})$ as so does the item $h_{n_{\beta(\mu)-1}}^{(1)}$ of $\Omega_{1}$.

Notice that we have shown that for any item $h_{i}^{(0)}$ covered by $\mu$, if $\mathfrak{l}(i)<\mathfrak{l}(i+1)$, then the tribe of $h_{i}^{(0)}$ is minimal and belongs to $h(\mathfrak{M})$. Furthermore, all the boundaries are introduced in items whose tribe is $\mathfrak{M}$. Hence for any $h_{i}^{(0)}$ covered by $\mu$ such that $\mathfrak{l}(i)=\mathfrak{l}(i+1)$, we have that its tribe dominates the minimal tribe $\mathfrak{M}$ of $h_{1}^{(0)}$.

Remark 3.19 From the proof of Lemma 3.18, it follows that, under the assumptions of the lemma, the image of the minimal item $h_{\mathfrak{l}(2)}^{(0)}$ of $\Omega_{0}$ under $\pi\left(v_{0}, v_{1}\right)$ is $h_{\mathfrak{l}(2)}^{(1)} h_{n_{2}}^{(1)}$ and both of the items $h_{\mathfrak{l}(2)}^{(1)}, h_{n_{2}}^{(1)}$ are minimal.

Lemma 3.20 Let $\Omega_{0}$ be a generalised equation of type 12 that repeats infinitely many times in the infinite branch. Then, in the infinite branch, all bases of $\Omega_{0}$, except, perhaps, bases $v$ contained in the long base $\lambda$ so that $\beta(\lambda)=\rho_{A}$, are transferred infinitely many times.

Proof Notice that, without loss of generality, we can assume that if a base is transferred, then it is transferred infinitely many times in an infinite branch.

Consider the long base $\lambda$ of $\Omega_{0}$ so that $\beta(\lambda)=\rho_{A}$. Let $C(\lambda)$ be the set of bases contained in $\lambda$.

If either $\lambda$ or any of the bases in $C(\lambda)$ is the carrier base or is transferred in some generalised equation of the infinite branch, say $\Omega_{k}$, then $h_{1}^{(k)}$ is an item with boundaries inside one of the items $h_{\alpha(\lambda)}^{(0)}, \ldots, h_{\beta(\lambda)}^{(0)}$. Hence, the section $[1, \alpha(\lambda)]$ of $\Omega_{0}$ (and so all the bases in this section) has been transferred and the statement follows. Notice that since the base $\lambda$ is long, for a base contained in $\lambda$ to be a carrier base of a generalised equation $\Omega_{k}$, it is necessary that $\Delta(\lambda)$ or $\lambda$ be the carrier base of a generalised equation $\Omega_{l}$, where $l<k$.

Assume further that $\lambda$ and the bases from $C(\lambda)$ are neither carrier bases nor transfer bases. 
Without loss of generality, we may assume that there exists a base $\eta \in C(\lambda) \cup\{\lambda\}$ so that $\Delta(\eta)$ is the carrier of some $\Omega_{k}$. Indeed, suppose that $\Delta(\eta)$ is neither carrier nor transfer base for all $\eta \in C(\lambda) \cup\{\lambda\}$. In this case, the section $[\alpha(\lambda), \beta(\lambda)]$ can be declared nonactive and we can set $\rho_{A}$ to be $\alpha(\lambda)$. Similarly, if some base $\Delta(\eta)$ is transferred but for all generalised equations we have $\beta(\Delta(\eta))-\alpha(\Delta(\eta))=1$, then the section $[\alpha(\lambda), \beta(\lambda)]$ can be declared nonactive and we can set $\rho_{A}$ to be $\alpha(\lambda)$. Finally, assume that a base $\Delta(\eta)$ is transferred and for some generalised equation we have that $\beta(\Delta(\eta))-\alpha(\Delta(\eta))>1$. Since $\Delta(\eta)$ is transferred infinitely many times and transfer bases are always short, it follows that before being transferred, the base $\Delta(\eta)$ must be a carrier base (since this is the only way a long base can become short). Therefore, there exists a base $\eta \in C(\lambda) \cup\{\lambda\}$ so that $\Delta(\eta)$ is the carrier of some $\Omega_{k}$.

Note that since the branch is infinite, every $\mu$-tying introduces a new boundary. Since there exists a base $\eta \in C(\lambda)$ so that $\Delta(\eta)$ is the carrier of some generalised equation, say $\Omega_{k}$, at this step the new boundaries are introduced in the section $[\alpha(\lambda), \beta(\lambda)]$. Hence, for the generalised equation $\Omega_{0}$ to repeat, there must exist $l$ so that $\Delta(\lambda)$ is the carrier of $\Omega_{l}$.

Notice that, in particular, we have shown that in the assumptions of the lemma, either $\lambda$ or its dual $\Delta(\lambda)$ is a carrier base.

Suppose that $\varepsilon(\lambda) \cdot \varepsilon(\Delta(\lambda))=-1$. Then, in the generalised equation $\Omega_{l+1}$ we have $\beta(\lambda)<\rho_{A}$. Hence, for the generalised equation $\Omega_{0}$ to repeat, the base $\lambda$ has to be transferred. In this case, we conclude that all the bases of $\Omega_{0}$, except, perhaps, bases $v$ contained in a base $\lambda$ so that $\beta(\lambda)=\rho_{A}$, are transferred infinitely many times.

Suppose now that $\varepsilon(\lambda) \cdot \varepsilon(\Delta(\lambda))=1$. Let $v$ be the (uniquely defined) transfer base of $\Omega_{l}$ so that $\alpha(v)=1$. Since the base $v$ is a transfer base of $\Omega_{l}$, it is transferred infinitely many times in an infinite branch and hence for some generalised equation, say $\Omega_{k}$, the item $h_{1}^{(k)}$ is an item with boundaries inside one of the items $h_{\alpha(\lambda)}^{(0)}, \ldots, h_{\beta(\lambda)}^{(0)}$. Hence, the section $[1, \alpha(\lambda)]$ of $\Omega_{0}$ (and so all the bases in this section) has been transferred and the statement follows.

Lemma 3.21 Let $\Omega$ be a generalised equation of type 12 that repeats infinitely many times in the infinite branch and let $h_{1}$ be minimal. Then, for every long base $\lambda$, the items $h_{\alpha(\lambda)}$ and $h_{\beta(\lambda)-1} \neq h_{\rho_{A}-1}$ are minimal.

In other words, all items that are not covered by a short base are minimal and belong to the same minimal tribe $\mathfrak{M}$ and all the other items belong to tribes that dominate $\mathfrak{M}$.

Proof By Lemma 3.18, if $\Omega_{1}$ is obtained from $\Omega$ by a (complete) entire transformation D5, then the item $h_{1}^{(1)}$ of $\Omega_{1}$ is also minimal. Hence, in the infinite branch, 
the items $h_{1}^{(i)}$ and $h_{\beta\left(\mu_{i}\right)-1}^{(i)}$ are always minimal and the items $h_{j}, 1<j<\beta\left(\mu_{i}\right)-1$ belong to tribes that dominate the tribe of $h_{1}^{(i)}$, where $\mu_{i}$ is the carrier of $\Omega_{i}$.

Let $\lambda$ be the (uniquely defined) long base so that $\beta(\lambda)=\rho_{A}$. We now consider an arbitrary long base $\kappa$ of the generalised equation $\Omega$ distinct from $\lambda$. To prove the lemma, it suffices to show that $h_{\beta(\kappa)-1}$ is minimal.

For every generalised equation $\Omega_{i}$ we have that either

(I) the boundary 1 of $\Omega_{i}$ corresponds to a boundary $j_{i}$ of $\Omega, j_{i} \geq \beta(\kappa)$ in $\Omega$, or

(II) there is a base $v_{i}$ of $\Omega_{i}$ so that $\beta\left(v_{i}\right)$ corresponds to the boundary $\beta(\kappa)$ of $\Omega$.

Furthermore, since $\kappa \neq \lambda$, by Lemma 3.20, there exists $N$ so that for all $n \geq N$ the generalised equation $\Omega_{n}$ satisfies case (I), and for all $m<N$ the generalised equation $\Omega_{m}$ satisfies case (II).

Notice that the boundary $\beta\left(\mu_{N-1}\right)$ of $\Omega_{N-1}$ corresponds to a boundary $j_{N}$ of $\Omega$, so that $j_{N} \geq \beta(\kappa)$. Suppose that $j_{N}=\beta(\kappa)$ (ie the boundary $\beta\left(v_{N-1}\right)=\beta\left(\mu_{N-1}\right)$ corresponds to the boundary $\beta(\kappa)$ of $\Omega$ ). Then the boundary $\beta\left(\mu_{N-1}\right)-1$ of $\Omega_{N-1}$ corresponds to a boundary between $\beta(\kappa)-1$ and $\beta(\kappa)$ in $\Omega$. Since $h_{\beta\left(\mu_{N-1}\right)-1}^{(N-1)}$ is minimal, so is $h_{\beta(\kappa)-1}$.

If $j_{N}>\beta(\kappa)$ in $\Omega$ (ie $v_{N-1}$ is a transfer base of $\Omega_{N-1}$ ), then, it is not hard to see that the boundary $\alpha\left(\mu_{N-1}\right)$ of $\Omega_{N-1}$ corresponds to a boundary between $\beta(\kappa)-1$ and $\beta(\kappa)$ in $\Omega$. We conclude that $h_{\beta(\kappa)-1}$ is minimal.

Corollary 3.22 Let $\Omega$ be a generalised equation of type 12 that repeats infinitely many times in the infinite branch and let the item $h_{1}$ of $\Omega$ be a minimal item that belongs to the minimal tribe $\mathfrak{M}=t\left(h_{1}\right)$ of $\Omega$. Then the tribe $t\left(h_{i}\right)$ of any item $h_{i}$ of $\Omega$ dominates the tribe $t\left(h_{1}\right), i=2, \ldots, \rho_{A}-1$.

Proof By Lemma 3.20, all bases of $\Omega$ except, perhaps, bases $v$ contained in a base $\lambda$ so that $\beta(\lambda)=\rho_{A}$, are transferred infinitely many times. Hence, by Lemma 3.21, it now follows that the tribes of all the items $h_{i}, i=1, \ldots, \alpha(\lambda)-1$ of $\Omega$ dominate the tribe $t\left(h_{1}\right)$, where $\lambda$ is the (uniquely defined) long base so that $\beta(\lambda)=\rho_{A}$.

Since the base $\Delta(\lambda)$ is not contained in $\lambda$, so the tribe of every item that belongs to $\Delta(\lambda)$ dominates the tribe $t\left(h_{1}\right)$. It follows that the base $\Delta(\lambda)$, and so $\lambda$, belong to a tribe that dominates $t\left(h_{1}\right)$. Therefore, the tribe of every item that belongs to $\lambda$ dominates $t\left(h_{1}\right)$. 
Definition 3.23 It follows from Corollary 3.22 that if $\Omega$ is a generalised equation of type 12 that repeats infinitely many times in the infinite branch, then there is only one minimal tribe in $\Omega$, ie $|\mathfrak{m}(\Omega)|=1$. We further denote this minimal tribe by $\mathfrak{M}$.

Definition 3.24 Let $\Omega$ be a generalised equation that repeats infinitely many times in an infinite branch of type 12 and such that the item $h_{1}$ of $\Omega$ is minimal and belongs to the minimal tribe $\mathfrak{M}=t\left(h_{1}\right)$ of $\Omega$. We call such a generalised equation tribal generalised equation of type 12 . Note that by Corollary 3.17, tribal generalised equations of type 12 exist in any infinite branch of type 12 .

We summarise the discussion of this section in the following lemma.

Lemma 3.25 [14, Lemma 7.11] Let $v_{0} \rightarrow v_{1} \rightarrow \cdots \rightarrow v_{n} \rightarrow \cdots$ be an infinite path in the tree $T(\Omega)$, where $\operatorname{tp}\left(v_{i}\right)=12$ for all $i$, and $\Omega_{v_{0}}, \Omega_{v_{1}}, \ldots, \Omega_{v_{n}}, \ldots$ be the sequence of corresponding generalised equations. Then among $\left\{\Omega_{v_{i}}\right\}$ some tribal generalised equation of type 12 occurs infinitely many times. Furthermore, if $\Omega_{v_{k}}=\Omega_{v_{l}}$, then $\pi\left(v_{k}, v_{l}\right)$ is a $\mathbb{G}$-automorphism of the coordinate group $\mathbb{G}_{R\left(\Omega_{v_{k}}\right)}$ invariant with respect to the nonquadratic part.

3.3.2 Case 15 Let us now consider an infinite branch of type 15. If an infinite branch of type 15 has infinitely many generalised equations that contain nontrivial quadratic part (or, in other words, there are infinitely many consecutive leading quadratic bases), then the quadratic part of some generalised equation, say $\Omega$, repeats infinitely many times in the branch; see [14, Lemma 7.11]. By Corollary 3.17, in an infinite sequence of generalised equations (with nontrivial quadratic parts) there exists at least one whose quadratic part is tribal of type 12 , ie the corresponding quadratic part repeats infinitely many times in the infinite branch and $h_{1}$ belongs to the minimal tribe of the quadratic part.

Definition 3.26 A generalised equation in an infinite branch of type 15 is called tribal generalised equation of type 15 if either its quadratic part is tribal of type 12 or its quadratic part is trivial.

3.3.3 Linear case In the linear case, the following lemma is an immediate consequence of the construction of the tree $T^{\prime}$.

Lemma 3.27 [14, Lemma 7.9] Let $v_{0} \rightarrow v_{1} \rightarrow \cdots \rightarrow v_{n} \rightarrow \cdots$ be an infinite path in the tree $T^{\prime}(\Omega)$, where $7^{\prime} \leq \operatorname{tp}\left(v_{i}\right) \leq 10^{\prime}$ for all $i$, and let $\Omega_{v_{0}}, \Omega_{v_{1}}, \ldots, \Omega_{v_{n}}, \ldots$ be the sequence of corresponding generalised equations. Then among $\left\{\Omega_{v_{i}}\right\}$ some generalised equation $\Omega_{v_{k}}$ occurs infinitely many times. If $\Omega_{v_{k}}=\Omega_{v_{l}}$, then $\pi\left(v_{k}, v_{l}\right)$ is a $\mathbb{G}-$ automorphism of $\mathbb{G}_{R\left(\Omega_{v_{k}}\right)}$ invariant with respect to the kernel of $\Omega_{v_{k}}$. Furthermore, all items that do not belong to the kernel $\operatorname{Ker}\left(\Omega_{v_{k}}\right)$ belong to a minimal tribe. 
Definition 3.28 A (constrained) generalised equation $\Omega$ of type $i, i \in\left\{7^{\prime}, \ldots, 10^{\prime}\right\}$ that appears infinitely many times in an infinite branch of linear type is called tribal linear generalised equation.

3.3.4 The tribal finite tree $\boldsymbol{T}_{\mathbf{0}}(\boldsymbol{\Omega})$ Repeating the argument of [14, Chapter 7] and using Lemmas 3.27 and 3.25 instead of Lemmas 7.9 and 7.11 of [14], we obtain the following proposition.

Proposition 3.29 [14, Proposition 7.1] For a (constrained) generalised equation $\Omega=\Omega_{v_{0}}$, one can effectively construct a finite oriented rooted at $v_{0}$ tree $T_{0}, T_{0}=$ $T_{0}\left(\Omega_{v_{0}}\right)$ such that:

(1) The tree $T_{0}$ is a subtree of the tree $T^{\prime}(\Omega)$.

(2) To the root $v_{0}$ of $T_{0}$ we assign a recursive group of automorphisms $\operatorname{Aut}(\Omega)$ related to tribal generalised equations of type 12,15 and linear in the tree $T_{0}$.

(3) For any solution $H$ of a generalised equation $\Omega$ there exists a leaf $w$ of the tree $T_{0}(\Omega), \operatorname{tp}(w)=1,2$, and a solution $H^{[w]}$ of the generalised equation $\Omega_{w}$ such that

- $H^{[w]}<\operatorname{Aut}(\Omega) H$ (see [14, page 109] for notation);

- if $\operatorname{tp}(w)=2$ and the generalised equation $\Omega_{w}$ contains nonconstant nonactive sections, then either

* there exists a period $P$ such that $H^{[w]}$ is periodic with respect to the period $P$ and the generalised equation $\Omega_{w}$ is either singular of strongly singular with respect to the periodic structure $\mathcal{P}\left(H^{[w]}, P\right)$ or

* the section has been transferred to the nonactive part by an auxiliary base.

Recall that in the process of constructing the tribal tree $T_{0}(\Omega)$, we duplicate some infinite branches of type 12; see Remark 3.11. This splitting allows us to have extra assumptions on solutions that factor through a given branch. We record this fact in the remark below.

Remark 3.30 Let $\Omega_{k}$ be a tribal generalised equation of type 12 in $T_{0}(\Omega)$. If $h_{i}^{(k)} \in \mathcal{F}\left(\Omega_{k}\right)$, then

$$
\varphi \pi\left(v_{k}, v_{k+1}\right)\left(h_{i}^{(k)}\right)=w\left(h^{(k+1)}\right),
$$

where $w\left(h^{(k+1)}\right)$ is a word in items from $\Omega_{k+1}$ such that for a solution $H^{(k)}=$ $\varphi \pi\left(v_{k}, v_{k+1}\right) H^{(k+1)}$, we have that $H_{i}^{(k)}=w\left(H^{(k+1)}\right)$. 


\subsection{The tribal tree $T_{\text {sol }}(\Omega)$}

This section follows the constructions presented in [14, Chapters 8 and 9]. Our main goal is to construct a finite tribal tree $T_{\mathrm{dec}}(\Omega)$ that contains the tribal tree $T_{0}(\Omega)$ as a subtree and for which all the epimorphisms from the root to the leaves are proper. In order to do so, we need to take care of the new nonactive sections that are created by the auxiliary bases introduced in Case 6.5. Once we proved the existence of such tribal tree $T_{\mathrm{dec}}(\Omega)$, the construction of the tribal solution tree is exactly the same as in [14, Chapter 9]. Namely, we proceed by constructing the tree $T_{\mathrm{dec}}$ for each leaf of the tree $T_{\operatorname{dec}}(\Omega)$ and use the fact that partially commutative groups are equationally Noetherian to conclude that this construction terminates.

Proposition 3.31 [14, Proposition 8.1] For a (constrained) generalised equation $\Omega=\Omega_{v_{0}}$, one can effectively construct a finite oriented rooted at $v_{0}$ tree $T_{\mathrm{dec}}, T_{\mathrm{dec}}=$ $T_{\mathrm{dec}}\left(\Omega_{v_{0}}\right)$ such that:

(1) The tribal tree $T_{0}(\Omega)$ is a subtree of the tribal tree $T_{\mathrm{dec}}$.

(2) To every vertex $v$ of $T_{\mathrm{dec}}$ we assign a recursive group of automorphisms $A\left(\Omega_{v}\right)$ (related to tribal generalised equations).

(3) For any solution $H$ of a generalised equation $\Omega$ there is a leaf $u$ of the tree $T_{\mathrm{dec}}$, $\operatorname{tp}(u)=1,2$, and a solution $H^{[u]}$ of the generalised equation $\Omega_{u}$ such that

- $\pi_{H}=\sigma_{0} \pi\left(v_{0}, v_{1}\right) \sigma_{1} \ldots \pi\left(v_{n-1}, u\right) \sigma_{n} \pi_{H^{[u]}}$, where $\sigma_{i} \in A\left(\Omega_{v_{i}}\right)$;

- if $\operatorname{tp}(u)=2$, then all nonactive sections of $\Omega_{u}$ are constant sections.

Proof By Proposition 3.29, if the type of a leaf $w$ of the tribal tree $T_{0}(\Omega)$ is 2 and the generalised equation $\Omega_{w}$ contains nonconstant nonactive sections, then either

- there exists a period $P$ such that $H^{[w]}$ is periodic with respect to the period $P$ and the generalised equation $\Omega_{w}$ is either singular of strongly singular with respect to the periodic structure $\mathcal{P}\left(H^{[w]}, P\right)$ or

- the section has been transferred to the nonactive part by an auxiliary base.

The treatment of the former case, namely when the nonactive section has a singular or strongly singular periodic structure is exactly the same as in [14, Section 8.1].

To deal with the nonactive sections transferred by auxiliary bases, we proceed by induction on the height of tribes.

If the tribe of an auxiliary base that transferred the section $\sigma$ is maximal, then we declare the section $\sigma$ active in $\Omega_{w}$ and construct the tribal tree $T_{0}\left(\Omega_{w}\right)$. Notice that 
since every item in the section $\sigma$ (and so in the active part of $\Omega_{w}$ ) is maximal, by Lemma 3.4 so are all the items in all the generalised equations that appear in the tree $T^{\prime}\left(\Omega_{w}\right)$. It follows that no vertex in the tree $T^{\prime}\left(\Omega_{w}\right)$ is of type 6.5. Therefore, there are no more new auxiliary bases added in the construction of the tree $T_{0}\left(\Omega_{w}\right)$.

Furthermore, if the tree $T_{0}(\Omega)$ has leaves of type 2 that are not periodised with respect to a singular or strongly singular periodic structure, then the corresponding nonactive sections have been transferred by auxiliary bases in the section $\sigma$ and so these active sections also belong to a maximal tribe, but contain strictly less (than $\sigma$ ) auxiliary bases (since no auxiliary bases appear in the process and the carrier is an auxiliary base and is removed). We therefore can proceed by induction on the number of auxiliary bases. If there are no auxiliary bases in the nonactive section $\sigma$ that has been transferred by a maximal auxiliary base, then all leaves of the tree $T_{0}\left(\Omega_{w}\right)$ of type 2 correspond to generalised equations periodised with respect to singular or strongly singular periodic structures and we can construct the tree $T_{\mathrm{dec}}\left(\Omega_{w}\right)$ just as in [14].

We have shown that if the auxiliary base belongs to a maximal tribe, then one can construct the tribal tree $T_{\mathrm{dec}}$.

Since, by construction, auxiliary bases belong to a tribe that strictly dominates a minimal tribe of $\Omega$, it follows that the minimal tribe of a nonactive section transferred by an auxiliary base is not minimal. Hence, by induction we can construct the tree $T_{\operatorname{dec}}\left(\Omega_{w}\right)$ where $\Omega_{w}$ is a generalised equation of type 2 in $T_{0}(\Omega)$ and is not periodised.

Define $T_{\text {dec }}(\Omega)$ as the tree obtained from $T_{0}(\Omega)$ by gluing to each leaf $w$ of type 2 the corresponding tribal tree $T_{\mathrm{dec}}\left(\Omega_{w}\right)$. It is straightforward to check that the tree $T_{\operatorname{dec}}(\Omega)$ satisfies the required properties.

We now repeat the argument given in [14, Chapter 9] and use the fact that partially commutative groups are equationally Noetherian to construct the tribal solution tree from the tribal tree $T_{\mathrm{dec}}(\Omega)$. We summarise the main result of this section in the following theorem.

Theorem 3.32 [14, Theorem 9.2] Let $\Omega=\Omega(h)$ be a constrained generalised equation in variables $h$. Let $T_{\text {sol }}(\Omega)$ be the solution tree for $\Omega$. Then the following statements hold.

(1) For any solution $H$ of the generalised equation $\Omega$ there exist: a path $v_{0} \rightarrow$ $v_{1} \rightarrow \cdots \rightarrow v_{n}=v$ in $T_{\mathrm{sol}}(\Omega)$ from the root vertex $v_{0}$ to a leaf $v$, a sequence of automorphisms $\sigma=\left(\sigma_{0}, \ldots, \sigma_{n}\right)$, where $\sigma_{i} \in A\left(\Omega_{v_{i}}\right), \Omega_{v_{i}}$ are tribal generalised equations and a solution $H^{(v)}$ associated to the vertex $v$, such that

$$
\pi_{H}=\Phi_{\sigma, H^{(v)}}=\sigma_{0} \pi\left(v_{0}, v_{1}\right) \sigma_{1} \ldots \pi\left(v_{n-1}, v_{n}\right) \sigma_{n} \pi_{H^{(v)}} .
$$


(2) For any path $v_{0} \rightarrow v_{1} \rightarrow \cdots \rightarrow v_{n}=v$ in $T_{\text {sol }}(\Omega)$ from the root vertex $v_{0}$ to a leaf $v$, any sequence of automorphisms $\sigma=\left(\sigma_{0}, \ldots, \sigma_{n}\right), \sigma_{i} \in A\left(\Omega_{v_{i}}\right)$, and any solution $H^{(v)}$ associated to the vertex $v$, the homomorphism $\Phi_{\sigma, H^{(v)}}$ is a solution of $\Omega$. Moreover, every solution of $\Omega$ can be obtained in this way.

\section{Structure of coordinate groups}

As we discussed in the previous section, a key step in our construction of graph towers is the analysis of the structure of coordinate groups assigned to vertices of the solution tree. In general, the structure of a coordinate group does not depend only on the type of the generalised equation itself, but rather on the existence of some dynamical behaviour associated to it. Deeper understanding of these dynamics motivated and piloted the changes made to the process described in [14] and led us to the concept of tribal generalised equation and the construction of the tribal solution tree.

In this section, we show that the coordinate groups of tribal generalised equations of type 12, 15 and linear type do have a clear algebraic structure, that is, they split as amalgamated products while preserving the underlying structure of graph products.

Note that in this section we primarily work with the groups $G_{\Omega}$. However, the notions of canonical homomorphisms, fundamental branch etc are defined using coordinate groups of generalised equations. All these notions carry over from coordinate groups to the groups $G_{\Omega}$ and we use them in this context. For instance, by construction, the canonical homomorphism $\pi\left(v_{0}, v_{1}\right): G_{R\left(\Omega_{0}\right)} \rightarrow G_{R\left(\Omega_{1}\right)}$ is induced by a homomorphism from $G_{\Omega_{0}}$ to $G_{\Omega_{1}}$ which, abusing the notation, we also denote by $\pi\left(v_{0}, v_{1}\right)$. Similarly, any solution $H$ of $\Omega$ is induced by a homomorphism from $G_{\Omega}$ to $\mathbb{G}$, which we also denote by $H$.

\subsection{Structure of the quadratic part}

Let $\Omega=\langle\Upsilon, \Re \Upsilon\rangle$ be a tribal generalised equation of type 12 . Denote by $\mathcal{B S}_{A}(\Omega)$ the set of all active bases of the generalised equation $\Omega$. Our next goal is to show the set

$$
\left\{h(\eta) \mid \eta \in \mathcal{B S}_{A}(\Omega)\right\}
$$

is a generating set of the subgroup of $G_{\Omega}$ generated by the active items of $\Omega$ (ie the subgroup generated by the items from the quadratic section of $\Omega$ ). In this new generating set, we prove that this subgroup is Tietze-equivalent to a one-relator quotient of a partially commutative group, where the relation is a quadratic word. 
To simplify the notation, below we sometimes write $\eta$ instead of $h(\eta)$. Firstly, we use induction to define the map $\psi$ from the subgroup generated by the items of the quadratic part of $\Upsilon$ to the subgroup $\left\langle\mathcal{B S}_{A}(\Omega)\right\rangle$ of $G_{\Upsilon}$ as follows.

Recall that if $\mu_{1}$ is the long leading base, then every item $h_{i}, i=1, \ldots, \beta\left(\mu_{1}\right)-2$ is covered by a short base $v_{i}$. Then, for every item $h_{i}, i=1, \ldots, \beta\left(\mu_{1}\right)-2$, we set $\psi\left(h_{i}\right)=v_{i}$.

Notice that

$$
h_{\beta\left(\mu_{1}\right)-1}=h_{\beta\left(\mu_{1}\right)-2}^{-1} \cdots h_{2}^{-1} h_{1}^{-1} \mu_{1} .
$$

We set

$$
\psi\left(h_{\beta\left(\mu_{1}\right)-1}\right)=\psi\left(h_{\beta\left(\mu_{1}\right)-2}^{-1} \cdots h_{2}^{-1} h_{1}^{-1}\right) \mu_{1} .
$$

Note that the image of the item $h_{\beta\left(\mu_{1}\right)-1}$ is a word $w_{h_{\beta\left(\mu_{1}\right)-1}}$ in the bases $v_{i}$ so that $\alpha\left(v_{i}\right)<\beta\left(\mu_{1}\right)-1$ and every such base occurs in $w_{h_{\beta\left(\mu_{1}\right)-1}}$ precisely once.

Suppose that the map $\psi$ is defined for all the items $h_{i}, i<k$, and that if an item $h_{i}$ is covered only by long bases, then the image $\psi\left(h_{i}\right)$ is a word $w_{h_{i}}$ in the bases $v$ so that $\alpha(v)<i$ and every such base occurs in $w_{h_{i}}$ precisely once.

We define $\psi\left(h_{k}\right)$ recursively. If $h_{k}$ is covered by a short base $v_{k}$, then we set $\psi\left(h_{k}\right)=v_{k}$. Otherwise, $h_{k}$ is only covered by (two) long bases $\kappa_{1}$ and $\kappa_{2}$, where $\alpha\left(\kappa_{2}\right)=\beta\left(\kappa_{1}\right)-1=k$. Then we define

$$
\begin{aligned}
\psi\left(h_{k}\right) & =\psi\left(h_{k-1}^{-1} \cdots h_{1}^{-1} h_{1} \cdots h_{\alpha\left(\kappa_{1}-1\right)}\right) \kappa_{1} \\
& =\psi\left(\left(h_{k-1}^{-1} \cdots h_{\alpha\left(\kappa_{1}\right)}^{-1}\right) \kappa_{1}=v_{k-1}^{-1} \cdots v_{\alpha\left(\kappa_{1}\right)+1}^{-1} \psi\left(h_{\alpha\left(\kappa_{1}\right)}^{-1}\right) \kappa_{1} .\right.
\end{aligned}
$$

Since $\alpha\left(\kappa_{1}\right)<k$ and the item $h_{\alpha\left(\kappa_{1}\right)}$ is covered by two long bases, it follows by induction assumption that $\psi\left(h_{\alpha\left(\kappa_{1}\right)}\right)$ is a word in the bases $v$ so that $\alpha(v)<\alpha\left(\kappa_{1}\right)$, and every such base occurs in this word precisely once. It follows that $\psi\left(h_{k}\right)$ is a word in the bases $v$ so that $\alpha(v)<k$, and every such base occurs in this word precisely once.

Example 4.1 Let $\Omega$ be the generalised equation given on Figure 1. Then

$$
\begin{gathered}
\psi\left(h_{1}\right)=v, \quad \psi\left(h_{2}\right)=\eta, \quad \psi\left(h_{3}\right)=\eta^{-1} v^{-1} \mu, \quad \psi\left(h_{4}\right)=\Delta(\lambda), \\
\psi\left(h_{5}\right)=\Delta(\lambda)^{-1} \mu^{-1} v \eta \Delta(v), \quad \psi\left(h_{6}\right)=\lambda, \quad \psi\left(h_{7}\right)=\Delta(\eta) .
\end{gathered}
$$

We now determine the presentation of the subgroup generated by active items of the quadratic part of $\Upsilon$ in the generators $\mathcal{B S}_{A}(\Omega)$. 
Note that, by Lemma 3.10, every boundary of $\Omega$ touches precisely two bases, ie for every boundary $i, 1<i<\rho$, there exist a base $v_{i}$ such that $\alpha\left(v_{i}\right)=i$ and a base $\lambda_{i}$ such that $\beta\left(\lambda_{i}\right)=i$. Therefore we can define the words

$$
W_{1}=\mu_{1} v_{\beta\left(\mu_{1}\right)} v_{\beta\left(v_{\beta\left(\mu_{1}\right)}\right)} \cdots v_{m} v_{\beta\left(v_{m}\right)},
$$

where $\beta\left(v_{\beta\left(v_{m}\right)}\right)=\rho_{\Omega}$ and

$$
W_{2}=v_{1} v_{\beta\left(v_{1}\right)} v_{\beta\left(v_{\beta\left(v_{1}\right)}\right)} \cdots v_{n} v_{\beta\left(v_{n}\right)},
$$

where $\beta\left(v_{\beta\left(v_{n}\right)}\right)=\rho_{\Omega}$. We set

$$
W=W_{1} W_{2}^{-1} .
$$

For each item $h_{i}, i=\rho_{A}, \ldots, \rho_{\Omega}-1$, ie for items that do not belong to the active quadratic section, we set $\psi\left(h_{i}\right)=h_{i}$.

Example 4.2 Let $\Omega$ be the generalised equation given on Figure 1. Then

$$
W_{1}=v \eta \Delta(\nu) \lambda \Delta(\eta), \quad W_{2}=\mu \Delta(\lambda) \Delta(\mu) .
$$

Lemma 4.3 In the above notation, the map $\psi$ induces an isomorphism from $G_{\Upsilon}$ to the group

$$
\begin{aligned}
& \mathcal{K}_{\Upsilon}=\left\langle\mathcal{B S}_{A}(\Omega), h_{\rho_{A}}, \ldots, h_{\rho_{\Omega}-1}\right| W=1, \\
&\left.\eta^{\varepsilon(\eta)} \Delta(\eta)^{-\varepsilon(\Delta(\eta))}=1, v^{\varepsilon(v)} h(\Delta(v))^{-\varepsilon(\Delta(v))}, R^{\prime}\right\rangle,
\end{aligned}
$$

where $\eta$ runs over the set of quadratic bases, $v$ runs over the set of quadratic-coefficient bases and $R^{\prime}$ is the set of relations of the nonactive part, ie $R^{\prime}$ is the set of relations $h(\mu)^{\varepsilon(\mu)} h(\Delta(\mu))^{-\varepsilon(\Delta(\mu))}$, for all pairs of nonactive bases $\mu, \Delta(\mu)$.

Proof Straightforward computation shows that for every base $\eta$ that belongs to the quadratic part such that either $\eta$ is short or $\beta(\eta) \neq \rho_{A}$, we have $\psi(h(\eta))=\eta$. Indeed, if $\eta$ is short, then the statement is obvious. Let $\eta$ be a long base. Then the item $h_{\beta(\eta)-1}$ is covered by two long bases, $\eta$ and $\kappa$. We have

$$
\begin{aligned}
\psi(h(\eta)) & =\psi\left(h_{\alpha(\eta)}\right) \cdots \psi\left(h_{\beta(\eta)-2}\right) \psi\left(h_{\beta(\eta)-1}\right) \\
& =\psi\left(h_{\alpha(\eta)}\right) \cdots \psi\left(h_{\beta(\eta)-2}\right) \cdot v_{\beta(\eta)-2}^{-1} \cdots v_{\alpha(\eta)+1}^{-1} \psi\left(h_{\alpha(\eta)}^{-1}\right) \eta \\
& =\psi\left(h_{\alpha(\eta)}\right) \cdot v_{\alpha(\eta)+1} \cdots v_{\beta(\eta)-2} \cdot v_{\beta(\eta)-2}^{-1} \cdots v_{\alpha(\eta)+1}^{-1} \psi\left(h_{\alpha(\eta)}^{-1}\right) \eta=\eta .
\end{aligned}
$$

We conclude that $\psi$ maps $h(\eta)^{\varepsilon(\eta)} h(\Delta(\eta))^{-\varepsilon(\Delta(\eta))}$ to the identity in $\mathcal{K}_{\Upsilon}$ for all pairs of dual bases except for the pair $\lambda, \Delta(\lambda)$, where $\lambda$ is the (uniquely defined) long base 
so that $\beta(\lambda)=\rho_{A}$. For the pair $\lambda, \Delta(\lambda)$, we have

$$
\psi(h(\lambda))=\psi\left(h_{\alpha(\lambda)}\right) \cdots \psi\left(h_{\beta(\lambda)-1}\right)=\psi\left(h_{\alpha(\lambda)}\right) v_{\alpha(\lambda)+1} \cdots v_{\rho_{A}-1} .
$$

Thus, $\psi(h(\lambda))$ is the product of all bases $v$ of $\Omega$ so that $v \neq \lambda$. It follows that $\psi\left(h(\lambda)^{\varepsilon(\lambda)} h(\Delta(\lambda))^{-\varepsilon(\Delta(\lambda))}\right)$ is trivial in $\mathcal{K}_{\Upsilon}$ since $W=1$ and $\lambda^{\varepsilon(\lambda)} \Delta(\lambda)^{-\varepsilon(\Delta(\lambda))}=1$ are relations of $\mathcal{K}_{\Upsilon}$. We conclude that $\psi$ is a (surjective) homomorphism.

We define the map $\varrho: \mathcal{K}_{\Upsilon} \rightarrow G_{\Upsilon}$ by $\varrho(\eta)=h(\eta)$. The map $\varrho$ extends to a homomorphism. Straightforward verification shows that every relation of $\mathcal{K}_{\Upsilon}$ maps to the identity in $G_{\Omega}$ and that $\varrho \psi=\mathrm{id}$ and $\psi \varrho=\mathrm{id}$ and we conclude that $\psi$ is an isomorphism.

Let us now show how to extend the isomorphism $\psi$ to an isomorphism of $G_{\Omega}$.

Let $\Xi$ be the set of relations defined as follows.

- For every pair of items $h_{i}, h_{j}$ of $\Omega$ so that $\Re_{\Upsilon}\left(h_{i}, h_{j}\right)$ and $h_{i}, h_{j}$ are covered by short bases $v_{i}, v_{j}$, correspondingly, we set $\left[v_{i}, v_{j}\right]=1 \in \Xi$.

- For every pair of items $h_{i}, h_{j}$ of $\Omega$ so that $\Re_{\Upsilon}\left(h_{i}, h_{j}\right)$ and $h_{i}$ is covered by a short base $v_{i}$ and $h_{j}$ is a nonactive item, we set $\left[v_{i}, h_{j}\right]=1 \in \Xi$.

- For every pair of items $h_{i}, h_{j}$ of $\Omega$ so that $\Re \Upsilon\left(h_{i}, h_{j}\right)$ and $h_{i}$ is covered only by long bases and $h_{j}$ is a nonactive item, we set $\left[\eta, h_{j}\right]=1 \in \Xi$, for all bases $\eta$ in the quadratic section.

- For every pair of items $h_{i}, h_{j}$ of $\Omega$ so that $\Re_{\Upsilon}\left(h_{i}, h_{j}\right)$ and $h_{i}$ and $h_{j}$ belong to the nonactive part, we set $\left[h_{i}, h_{j}\right]=1 \in \Xi$.

Lemma 4.4 Let $\Omega=\langle\Upsilon, \Re \Upsilon\rangle$ be a tribal generalised equation of type 12 . Then, in the above notation, the map $\psi$ induces an isomorphism from $G_{\Omega}$ to the group

$$
\begin{aligned}
\mathcal{K}=\left\langle\mathcal{B S}_{A}(\Omega), h_{\rho_{A}}, \ldots, h_{\rho_{\Omega}-1}\right| W=1, \eta^{\varepsilon(\eta)} \Delta(\eta)^{-\varepsilon(\Delta(\eta))}=1, & \left.v^{\varepsilon(v)} h(\Delta(v))^{-\varepsilon(\Delta(v))}, R^{\prime}, \Xi\right\rangle,
\end{aligned}
$$

where $\eta$ runs over the set of quadratic bases, $v$ runs over the set of quadratic-coefficient bases and $R^{\prime}$ is the set of relations of the nonactive part, ie $R^{\prime}$ is the set of relations $h(\mu)^{\varepsilon(\mu)} h(\Delta(\mu))^{-\varepsilon(\Delta(\mu))}$, for all pairs of nonactive bases $\mu, \Delta(\mu)$.

Proof By Lemma 4.3, the map $\psi$ induces an isomorphism from $G_{\Upsilon}$ to $\mathcal{K}_{\Upsilon}$. Observe that if an item $h_{i}$ belongs to two long bases, then, by Lemma 3.13, it belongs to the minimal tribe. Since any other item $h_{j}$ from the quadratic part belongs to a tribe that dominates the tribe of $h_{i}$, it follows that $h_{j} \notin \Re \Upsilon\left(h_{i}\right)$, ie if $\Re_{\Upsilon}\left(h_{i}, h_{k}\right)$, then $h_{k}$ is nonactive. Now a straightforward verification shows that $\psi$ is an epimorphism. 
Furthermore, since, by Remark 3.3, the set $\Re \Upsilon$ is completed, it follows that $\psi$ is an isomorphism. Indeed, if an item $h_{i}$ belongs to two long bases, then by Lemma 3.21, it belongs to the minimal tribe. Since items of the quadratic part belong to tribes that dominate the minimal tribe and, by assumption, $\Re \Upsilon$ is completed, it follows that if $\Re_{\Upsilon}\left(h_{i}, h_{j}\right)$, then $\Re_{\Upsilon}\left(h_{i}, h_{k}\right)$ for all items $h_{k}$ from the quadratic part.

Our next goal is to show that under the above conditions, one can take the quadratic equation $W$ to the standard form. In order to do so, we replace the generalised equation $\Omega$ by another generalised equation, in which if an item belongs to a tribe which strictly dominates the minimal tribe, then this item is covered by a short quadraticcoefficient base.

Lemma 3.18 implies that items that belong to tribes which strictly dominate the minimal tribe belong to $\mathcal{F}\left(\Omega_{0}\right)$. By Remark 3.30, for any item $h_{i}^{(0)}$ that belongs to a tribe that strictly dominates $t\left(h_{1}^{(0)}\right)$, there is a word $w_{i}\left(h^{(1)}\right) \in G_{\Omega_{1}}$ so that for all solutions

$$
H^{(0)}=\varphi \pi\left(v_{0}, v_{1}\right) H^{(1)}
$$

we have that $\varphi \pi\left(v_{0}, v_{1}\right)\left(h_{i}\right)=w_{i}\left(h^{(1)}\right)$ and $H_{i}^{(0)}=H^{(1)}\left(w_{i}\right)$.

We replace $\Omega_{0}=\left\langle\Upsilon_{0}, \Re \Upsilon_{0}\right\rangle$ by a new generalised equation $\Omega_{0}^{\prime}=\left\langle\Upsilon_{0}^{\prime}, \Re \Upsilon_{0}^{\prime}\right\rangle$ constructed as follows. Replace the nonactive part of $\Omega_{0}$ by the nonactive part of $\Omega_{1}=\left\langle\Upsilon_{1}, \Re \Upsilon_{1}\right\rangle$. For any item $h_{i}^{(0)}$ in the active part that does not belong to the minimal tribe $t\left(h_{1}^{(0)}\right)$ let $w_{i}\left(h^{(1)}\right)=h_{i, 1}^{(1) \epsilon_{i, 1}} \cdots h_{i, k_{i}}^{(1) \epsilon_{i, k_{i}}}, \epsilon_{i, j} \in\{1,-1\}$.

Introduce new boundaries in the item $h_{i}^{(0)}$ so that

$$
h_{i}^{(0)}=h_{i, 1}^{(0)^{\prime} \epsilon_{i, 1}} \cdots h_{i, k_{i}}^{(0)^{\prime} \epsilon_{i, k_{i}}}, \quad \epsilon_{i, j} \in\{1,-1\}, j=1, \ldots, k_{i} .
$$

Erase the short base $\lambda$ of $\Omega_{0}$ covering $h_{i}^{(0)}$. We now introduce new bases $\lambda_{i, 1}, \ldots, \lambda_{i, k_{i}}$ along with the corresponding duals in such a way that $\lambda_{i, j}$ covers the item $h_{i, j}^{(0) \prime}, j=$ $1, \ldots, k_{i}$ and the dual $\Delta\left(\lambda_{i, j}\right)$ covers the item $h_{i, j}^{(1)}$ and $\varepsilon\left(\lambda_{i, j}\right)=\epsilon_{i, j}, \varepsilon\left(\Delta\left(\lambda_{i, j}\right)\right)=1$.

Note that, by Lemma 3.21, it follows that if a short base covers an item that belongs to a tribe which strictly dominates $t\left(h_{1}^{(0)}\right)$, then either it is a quadratic-coefficient base or it is quadratic and its dual is also a short base. Hence, the base $\Delta(\lambda)$ is a short base that covers an item that dominates $t\left(h_{1}^{(0)}\right)$.

The set $\Re^{\prime} \Upsilon_{0}^{\prime}$ of $\Omega_{0}^{\prime}$ is defined naturally:

$$
\begin{array}{rll}
\Re \Upsilon_{0}^{\prime}\left(h_{i, j}^{(0)^{\prime}}, h_{i^{\prime}, j^{\prime}}^{(0)}\right) & \text { if and only if } & \Re \Upsilon_{1}\left(h_{i, j}^{(1)}, h_{i^{\prime}, j^{\prime}}^{(1)}\right) \text { or } \quad \Re \Upsilon_{0}\left(h_{i}^{(0)}, h_{i^{\prime}}^{(0)}\right) . \\
\Re_{\Upsilon_{0}^{\prime}}\left(h_{i, j}^{(0)^{\prime}}, h_{i^{\prime}}^{(0)}\right) & \text { if and only if } & \Re \Upsilon_{0}\left(h_{i}^{(0)}, h_{i^{\prime}}^{(0)}\right) . \\
\Re_{\Upsilon_{0}^{\prime}}\left(h_{i, j}^{(0)^{\prime}}, h_{i}^{(1)}\right) & \text { if and only if } & \Re_{\Upsilon_{1}}\left(h_{i, j}^{(1)}, h_{i}^{(1)}\right) .
\end{array}
$$


The other relations in $\Re \Upsilon_{0}^{\prime}$ are naturally induced by $\Re \Upsilon_{0}$ and $\Re \Upsilon_{1}$. We assume $\Re \Upsilon_{0}^{\prime}$ is completed (see Remark 3.3). We conclude that:

- $\Omega_{0}^{\prime}$ is a generalised equation of type 12 .

- If $\varsigma: G_{\Omega_{0}} \rightarrow G_{\Omega_{0}^{\prime}}$ is a natural homomorphism, then any solution $H$ of the generalised equation $\Omega_{0}$ induces a solution $H^{\prime}$ of the generalised equation $\Omega_{0}^{\prime}$. In other words, the following diagram is commutative:

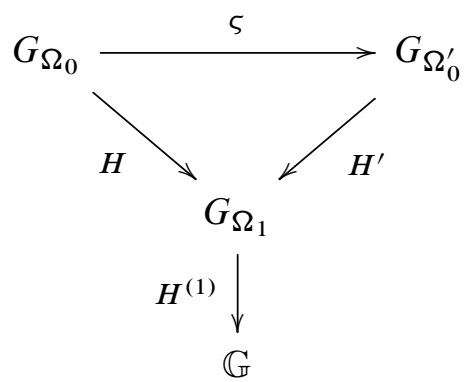

- $G_{\Omega_{1}}$ is a retraction of $G_{\Omega_{0}^{\prime}}$.

Remark 4.5 Note that if $v$ is an active base of $\Omega_{0}^{\prime}$, then either the tribe of every item $h_{i}^{(0) \prime}, i=\alpha(v), \ldots, \beta(v)-1$, covered by $v$ dominates $t\left(h_{1}^{(0)}\right)$ and at least one of the items belongs to the tribe $t\left(h_{1}^{(0)}\right)$ or else every item covered by $v$ belongs to a tribe that strictly dominates $t\left(h_{1}^{(0)}\right)$ and then the base $v$ is a short quadratic-coefficient base. In other words, in $\Omega_{0}^{\prime}$ the tribe of every quadratic base is the same minimal tribe and the tribes of the quadratic-coefficient bases dominate this minimal tribe.

Let

$$
\begin{aligned}
\mathcal{L}=\left\langle\left\{\eta \mid \eta \in \mathcal{B S}_{A}\left(\Omega_{v_{0}}^{\prime}\right)\right\}, h_{\rho_{A}}, \ldots, h_{\rho_{\Omega}-1}\right| \mu^{\varepsilon(\mu)} \Delta(\mu)^{-\varepsilon(\Delta(\mu))}=1, & \left.v^{\varepsilon(v)} h(\Delta(\nu))^{-\varepsilon(\Delta(v))}=1\right\rangle
\end{aligned}
$$

for all quadratic bases $\mu$ and all quadratic coefficient bases $v$. It is clear that $\mathcal{L}$ is a free group.

Let $\mathcal{M}$ be quotient of $\mathcal{L}$ by the set of commutators $\Xi^{\prime}$ defined analogously to $\Xi$; see (6). By definition, $\mathcal{M}$ is a free partially commutative group.

The word $W$ defined in (5) is a quadratic word in the free group $\mathcal{L}$. It can be taken to the surface relation form (9) or (10) by an automorphism of the free group $\mathcal{L}$; see Comerford and Edmunds [17]. 
Lemma 4.6 Let $\phi$ be the automorphism of the free group $\mathcal{L}$ that takes the quadratic equation $W=1$ to the normal form. Then $\phi$ induces an automorphism $\phi^{\prime}$ of the partially commutative group $\mathcal{M}$ that takes the quadratic equation $W=1$ to the normal form and fixes all the quadratic-coefficient bases and items $h_{i}, i=\rho_{A}, \ldots, \rho_{\Omega}-1$.

Proof Denote by $\operatorname{var}(W)$ the set of variables of $W=1$ that occur in $W$ exactly twice. By definition of $\mathcal{L}$ and $\mathcal{M}$ and by construction of $\Omega_{0}^{\prime}$, we get that if $x \in \operatorname{var}(W)$, then $x$ is a quadratic base of $\Omega_{0}^{\prime}$ and the tribe of $x$ is the minimal tribe $t\left(h_{1}^{(0)}\right)$.

Suppose that $W=A x B \times C$. Let $\phi_{x}$ be the automorphism of $\mathcal{L}$ induced by the map $x \rightarrow A^{-1} x A B^{-1}$. The automorphism $\phi_{x}$ transforms the word $W$ into

$$
\phi_{x}(W)=A A^{-1} x A B^{-1} B A^{-1} x A B^{-1} C=x^{2} A B^{-1} C .
$$

Every letter in $A$ and $B$ is either a variable of $W=1$ (then it corresponds to a quadratic base which belongs to $t\left(h_{1}^{(0)}\right)$ ) or it is a coefficient of $W=1$ (then it corresponds to a quadratic-coefficient base which belongs to a tribe that strictly dominates $t\left(h_{1}^{(0)}\right)$ ). Hence, by Laurence [42], it follows that $\phi_{x}$ induces an automorphism of $\mathcal{M}$.

Observe that $A B^{-1} C$ is a quadratic word in fewer variables than $W$. The statement now follows by induction.

Suppose now that every variable $x$ of $W$ occurs in it as $x$ and as $x^{-1}$. Let $W=$ $A x^{-1} B x C$, where the number of variables $|\operatorname{var}(B)|$ in $B$ is minimal among all such decompositions of $W$. In particular, it follows that $B$ is linear.

If $\operatorname{var}(B)=\varnothing$, then we consider the automorphism $\phi_{x}$ of $\mathcal{L}$ defined by the map $x \mapsto x C^{-1}$. Then $\phi_{x}(W)=A C x^{-1} B x$. As above, the automorphism $\phi_{x}$ induces an automorphism of $\mathcal{M}$. Note that the number of variables in $A C$ is strictly lower than that of $W$ and the statement, in this case, follows by induction.

Let $\operatorname{var}(B) \neq \varnothing$. Then $B=B_{1} y^{\delta} B_{2}$, where $\delta= \pm 1$, and neither $B_{1}$ nor $B_{2}$ contains $y^{ \pm 1}$. Applying the automorphism $y \mapsto y^{-1}$, if necessary, we may assume that $\delta=1$.

Consider the automorphism $\phi_{y}$ of $\mathcal{L}$ defined by $y \mapsto B_{1}^{-1} y B_{2}^{-1}$. Then $\phi_{y}(W)=$ $\phi_{y}(A) x^{-1} y x \phi_{y}(C)$. The variable $y^{-1}$ occurs either in $A$ or in $C$. We assume $y^{-1}$ occurs in $C$ (the other case is similar), ie $C=C_{1} y^{-1} C_{2}$. Then

$$
\phi_{y}(A)=A, \phi_{y}(C)=C_{1} B_{2} y^{-1} B_{1} C_{2} \quad \text { and } \quad \phi_{y}(W)=A x^{-1} y x C_{1} B_{2} y^{-1} B_{1} C_{2} .
$$

Applying the automorphism $\phi_{x}$ defined by the map $x \mapsto x\left(C_{1} B_{2}\right)^{-1}$, we get that

$$
\phi_{x} \phi_{y}(W)=A C_{1} B_{2} x^{-1} y x y^{-1} B_{1} C_{2} .
$$


Let $\phi_{1}$ be the automorphism that conjugates $x$ and $y$ by $A C B_{2}$. Then

$$
\phi_{1} \phi_{x} \phi_{y}(W)=x^{-1} y x y^{-1} B_{1} C_{2} A C_{1} B_{2} .
$$

As above, the automorphisms $\phi_{1}, \phi_{x}$ and $\phi_{y}$ induce automorphisms of $\mathcal{M}$. We now observe that $B_{1} C_{2} A C_{1} B_{2}$ is a quadratic word in fewer variables than $W$ and the statement now follows by induction.

Corollary 4.7 In the notation of Lemma 4.6, the automorphism $\phi^{\prime}$ induces an automorphism of the group $\mathcal{K}$ that takes the quadratic relation to the normal form. Therefore, if $\Omega=\langle\Upsilon, \Re \Upsilon\rangle$ is a tribal generalised equation of type 12, then $G_{\Omega}$ is isomorphic to the group

$$
\begin{aligned}
& \mathcal{K}=\left\langle\mathcal{B} \mathcal{S}_{A}(\Omega), h_{\rho_{A}}, \ldots, h_{\rho_{\Omega}-1}\right| W=1, \eta^{\varepsilon(\eta)} \Delta(\eta)^{-\varepsilon(\Delta(\eta))}=1, \\
&\left.v^{\varepsilon(v)} \Delta(\nu)^{-\varepsilon(\Delta(v))}=1, R^{\prime}, \Xi^{\prime}\right\rangle,
\end{aligned}
$$

where $\eta$ runs over the set of quadratic bases, $v$ runs over the set of quadratic-coefficient bases and $W$ is a quadratic word in the normal form

$$
\begin{aligned}
{\left[\eta_{1}, \eta_{2}\right] \cdots\left[\eta_{2 g-1}, \eta_{2 g}\right] \lambda_{2 g+1} \eta_{2 g+1} \cdots \lambda_{m}{ }^{\eta_{m}} } & \\
& =\pi\left(v_{0}, v_{1}\right)\left(\left[\eta_{1}, \eta_{2}\right] \cdots\left[\eta_{2 g-1}, \eta_{2 g}\right] \lambda_{2 g+1} \eta_{2 g+1} \cdots \lambda_{m}{ }^{\eta_{m}}\right)
\end{aligned}
$$

or

$$
\eta_{1}^{2} \cdots \eta_{2 g}^{2} \lambda_{2 g+1} \eta_{2 g+1} \cdots \lambda_{m} \eta_{m}=\pi\left(v_{0}, v_{1}\right)\left(\eta_{1}^{2} \cdots \eta_{2 g}^{2} \lambda_{2 g+1} \eta_{2 g+1} \cdots \lambda_{m}{ }^{\eta_{m}}\right)
$$

and where $R^{\prime}$ is the set of relations of the nonactive part, ie $R^{\prime}$ is the set of relations $\mu^{\varepsilon(\mu)} \Delta(\mu)^{-\varepsilon(\Delta(\mu))}$, where $\mu, \Delta(\mu)$ runs over the set of pairs of nonactive bases.

\subsection{Structure of the linear part}

Lemma 4.8 Let $\Omega=\langle\Upsilon, \Re \Upsilon\rangle$ be a tribal generalised equation of linear type $\left(7^{\prime}-10^{\prime}\right)$. Then, in the notation used in the description of the derived transformation D4 (see eg [14, Equations (4.1) and (4.2)]) the following isomorphisms hold:

$$
\begin{aligned}
G_{R(\Omega)} & \simeq \mathbb{G}\left[h_{1}, \ldots, h_{\rho_{\bar{\Omega}_{1}}}, z_{1}, \ldots, z_{l}\right] / R\left(\bar{\Omega}_{1} \cup\left\{\left[z_{k}, h_{j}\right] \mid \Re_{\Upsilon}\left(z_{k}, h_{j}\right)\right\}\right), \\
G_{R(\Omega)} & \simeq \mathbb{G}\left[h_{1}, \ldots, h_{\rho_{\bar{\Omega}^{\prime \prime}}}, z_{1}, \ldots, z_{l}\right] / R\left(\bar{\Omega}^{\prime \prime} \cup\left\{\left[z_{j}, h_{k}\right] \mid \Re_{\Upsilon^{\prime}}\left(z_{j}, h_{k}\right)\right\}\right) .
\end{aligned}
$$

Proof Indeed, to prove the lemma it suffices to observe that the relations $\left\{\left[h_{j}, w_{\mu}\right] \mid\right.$ $\left.\Re_{\Upsilon}\left(h_{j}, h_{i}\right)\right\}$ and $\left\{\left[z_{k}, w_{\mu}\right] \mid \Re_{\Upsilon}\left(z_{k}, h_{i}\right)\right\}$, and

$$
\begin{aligned}
\left\{\left[w_{\mu}{ }^{-1} h_{i}^{\prime}, h_{j}\right] \mid \Re \Upsilon_{\Upsilon_{1}}\left(h_{i}, h_{j}\right)\right\}, & \left\{\left[w_{\mu}{ }^{-1} h_{i}^{\prime}, z_{j}\right] \mid \Re \Upsilon_{\Upsilon_{1}}\left(h_{i}, z_{j}\right)\right\}, \\
\left\{\left[w_{\mu}, h_{j}\right] \mid \Re_{\Upsilon_{1}}\left(h_{i-1}, h_{j}\right)\right\}, & \left\{\left[w_{\mu}, z_{j}\right] \mid \Re_{\Upsilon_{1}}\left(h_{i-1}, z_{j}\right)\right\},
\end{aligned}
$$


belong to the normal closures of $\bar{\Omega}_{1} \cup\left\{\left[z_{k}, h_{j}\right] \mid \Re_{\Upsilon}\left(z_{k}, h_{j}\right)\right\}$ and $\bar{\Omega}^{\prime \prime} \cup\left\{\left[z_{j}, h_{k}\right] \mid\right.$ $\left.\Re_{\Upsilon^{\prime}}\left(z_{j}, h_{k}\right)\right\}$, correspondingly. This is immediate since, by the description of the cases $7^{\prime}-10^{\prime}$, the items that do not belong to the kernel belong to the minimal tribe and therefore the tribe of every item covered by an eliminable base dominates the tribe of the item $h_{i}$ (see the definition of eliminable base) and the set of relations $\Re \Upsilon$ is completed.

Corollary 4.9 [14, Lemma 4.7] In the notation of Lemma 4.8 and [14, Section 4.3], one has the isomorphism

$$
\mathbb{G}_{R(\Omega)} \simeq \mathbb{G}\left[h_{1}, \ldots, h_{\rho_{\overline{\operatorname{Ker}(\Omega)}}}, z_{1}, \ldots, z_{l}\right] / R(\overline{\operatorname{Ker}(\Omega)} \cup \mathcal{K}),
$$

where $\left\{z_{1}, \ldots, z_{l}\right\}$ is a set of free variables of $\operatorname{Ker}(\Omega)$, and $\mathcal{K}$ is a (computable) set of commutators of the form $\left[z_{i}, h_{j}\right]$ and $\left[z_{i}, z_{k}\right]$.

\section{Graph towers}

The goal of this section is to introduce the class of graph towers which is key for describing finitely generated fully residually partially commutative groups.

Graph towers are natural generalisations of $\omega$-residually free towers. Recall that a finitely generated group is an $\omega$-residually free tower if it belongs to the smallest class of groups containing all finitely generated free groups and (nonexceptional) surface groups, which is stable under taking free products, free extensions of centralisers, and attaching retracting surfaces along maximal cyclic subgroups.

As we mentioned in the introduction, $\omega$-residually free towers (alias NTQ-groups) are essential to characterise and describe the structure of the class of limit groups, as well as to classify groups elementarily equivalent to a free group; see [41; 54]. In the next section, we show that graph towers also characterise the class of fully residually partially commutative groups and it is left to see if they are going to play a similar role in the classification of groups elementarily equivalent to a given partially commutative group.

The notion of a graph tower is somewhat technical. Before turning our attention to the formal definition, let us review some particular cases to gain an intuition. Free extensions of centralisers are one of the basic operations for constructing limit groups (in some sense it is the main operation in constructing limit groups, since limit groups are also characterised as subgroups of iterated sequences of free extensions of centralisers; see [38]). As in the case of free groups, extensions of centralisers are also going to play an important role in characterising limit groups over partially commutative groups. 
Although it is a simple construction, it already demonstrates some of the subtleties that need to be taken into consideration.

As the following example shows, in general free extensions of centralisers are not discriminated by $\mathbb{G}$.

Example 5.1 Let $\mathbb{G}=F(a, b) \times F(c, d)$, and let $w=(a b, c d) \in \mathbb{G}$. Consider the extension of centraliser of the element $w$, that is, the group $H=\langle\mathbb{G}, t \mid[t, C(w)]=1\rangle$. It is easy to check that element $v=\left[a^{t}, c\right] \in H$ is nontrivial. However, for any homomorphism $\phi$ from $H$ to $\mathbb{G}$ which restricts to the identity on $\mathbb{G}$, we have that $\phi(t) \in C(w)=\langle a b\rangle \times\langle c d\rangle$ and so $\phi(v)=1$. Hence, $H$ is not discriminated (even separated) by $\mathbb{G}$.

However, we showed in $[13 ; 14]$ that any free extension of a centraliser of an irreducible element $b \in \mathbb{G}$ (see page 735 for definition) is discriminated by $\mathbb{G}$. (In fact, one can give a precise characterisation of the set of elements of a partially commutative group $\mathbb{G}$ whose extension of centraliser is discriminated by $\mathbb{G}$ in terms of their block decompositions.)

This particular case already shows that, on the one hand, there are some constraints on the type of elements whose centralisers need to be extended and, on the other hand, that one cannot expect to describe limit groups over partially commutative groups in terms of their abelian JSJ-decomposition (or their JSJ over small groups, or over slender groups) since in a free extension of a centraliser $H=\mathbb{G} * C(b)\langle C(b), t \mid[t, C(b)]\rangle$ the amalgamation is taken over a centraliser of an irreducible element, which in general, is a partially commutative group.

Another basic operation in the construction of $\omega$-residually free towers is "attaching a retracting surface". In the free group case, given a surface $\Sigma$ with boundary and Euler characteristic at most -2 or a punctured torus or a punctured Klein bottle one can attach it to a free group $F$ via a morphism $\rho: \pi_{1}(\Sigma) \rightarrow F$ with nonabelian image which is one-to-one on the restriction to the fundamental groups $C_{1}, \ldots, C_{n}$ of the boundary components of $\Sigma$, ie the limit group is the fundamental group of the graph of groups with two vertex groups $F$ and $\pi_{1}(\Sigma)$ and $n$ edge groups $C_{1}, \ldots, C_{n}$, where the two edge morphisms are the identity and the restriction of $\rho$. In this case, the surface naturally retracts to the free group and the discriminating family can be obtained from the retraction by precomposing it with modular automorphisms of the surface.

If one wants to follow this pattern in the case of partially commutative groups, then there are some necessary conditions to be imposed. As in the case of free extensions of centralisers, one is to impose that the cyclic fundamental groups $C_{1}, \ldots, C_{n}$ of 
the boundary components of the surface be mapped by the retraction $\rho$ to cyclic subgroups generated by irreducible elements $b_{1}, \ldots, b_{n}$ of the partially commutative group $\mathbb{G}$. Furthermore, in order to have "enough" automorphisms of the surface to obtain a discriminating family, we impose that the irreducible elements $b_{i}, i=1, \ldots, n$ commute with the same set of generators, ie $\mathbb{A}_{b}=\mathbb{A}\left(b_{i}\right)=\mathbb{A}\left(b_{j}\right)$, for all $1 \leq i, j \leq n$. Finally, since the discriminating family retracts the surface onto the group generated by $b_{i}, i=1, \ldots, n$, it is necessary to impose that the surface commute with $\mathbb{A}_{b}$ to assure discrimination. Under the conditions we imposed, it is not too difficult to see that the fundamental group of the graph of groups with two vertex groups $\mathbb{G}$ and $\pi_{1}(\Sigma) \times \mathbb{A}_{b}$ and $n$ edge groups $C_{1} \times \mathbb{A}_{b}, \ldots, C_{n} \times \mathbb{A}_{b}$, where the two edge morphisms are the identity and the restriction of $\rho$, is discriminated by $\mathbb{G}$. We will show that, basically, these sufficient conditions are necessary.

So, in view of the above discussion, we can say that, roughly speaking, graph towers are built hierarchically from the partially commutative group $\mathbb{G}$ by gluing retracting abelian groups and surface groups with the conditions that

- the abelian and surface groups are amalgamated along cyclic subgroups generated by "irreducible" elements and

- these abelian and surface groups commute with the centraliser of the subgroup onto which they retract to.

Although the idea is clear, the main technical problem in the formal definition of a graph tower is that, a priori, we do not have control on the structure of centralisers of elements of graph towers. In particular, it is not clear what an "irreducible" element is. Informally, given a limit group over a partially commutative group, one could define an irreducible element to be an element for which there exists a discriminating family that maps it into irreducible elements. But we can not define it this way either, since when constructing graph towers, we do not yet know that they are discriminated by $\mathbb{G}$ and in fact, it is only a posteriori that we are able to conclude that the homomorphisms induced by solutions that factor through the fundamental branch of the tribal solution tree discriminate the graph tower into $\mathbb{G}$ and so to deduce that our "irreducible" elements have this property.

To deal with this difficulty, graph towers are defined as a triple: the group $\mathfrak{T}$ (the graph tower), a partially commutative group $\mathbb{H}$ and an epimorphism from $\mathbb{H}$ to $\mathfrak{T}$. In what follows, we shall show that in fact both $\mathfrak{T}$ and $\mathbb{H}$ are fully residually $\mathbb{G}$ groups.

The partially commutative group $\mathbb{H}$ is defined via its commutation graph $\Gamma$. We subdivide the set of edges of the graph $\Gamma$ into two disjoint sets: $E_{c}(\Gamma)$ and $E_{d}(\Gamma)$. These two sets capture the different nature of commutation for the images of the 
elements of $\mathbb{H}$ in $\mathbb{G}$, namely two vertices $x$ and $y$ are joined by an edge from $E_{c}(\Gamma)$ if and only if for a discriminating family, $x$ and $y$ are sent to the same cyclic subgroup in $\mathbb{G}$; and two vertices $x$ and $y$ are joined by an edge from $E_{d}(\Gamma)$ if and only if for a discriminating family, the images of $x$ and $y$ disjointly commute. (We also prove the existence of a discriminating family with this property.)

Now we can define irreducible elements in $\mathfrak{T}$ as the images of irreducible elements in $\mathbb{H}$. More generally, we define coirreducible subgroups $\mathbb{K}$ as closed subgroups of $\mathbb{H}$ (ie $\mathbb{K}^{\perp \perp}=\mathbb{K}$ ) so that $\mathbb{K}^{\perp}$ is $E_{d}(\Gamma)$-directly indecomposable. The motivation behind this definition is as follows. Notice that since in a given finitely generated partially commutative group $\mathbb{G}$ there are finitely many different canonical parabolic subgroups, so for a canonical parabolic subgroup $\mathbb{K}$ of $\mathbb{H}$ (where $\mathbb{H}$ is discriminated by $\mathbb{G}$ ) there exists a subgroup $\mathbb{G}_{\mathbb{K}}$ of $\mathbb{G}$ so that for a discriminating family $\left\{\varphi_{i}\right\}$ we have that $\varphi_{i}(\mathbb{K})<\mathbb{G}_{\mathbb{K}}$ and for no proper subgroup $\mathbb{G}_{\mathbb{K}}^{\prime}$ of $\mathbb{G}_{\mathbb{K}}$ there exists such a discriminating family. We show that given a coirreducible subgroup $\mathbb{K}$ of $\mathbb{H}$, there exists a discriminating family so that $\mathbb{G}_{\mathbb{K}}$ is coirreducible in $\mathbb{G}$. In particular, $\mathbb{G}_{\mathbb{K}} \perp$ is a directly indecomposable subgroup of $\mathbb{G}$ and if $\mathbb{G}_{\mathbb{K}} \perp$ is not cyclic, then $C_{\mathbb{G}}\left(\mathbb{G}_{\mathbb{K}} \perp\right)=\mathbb{G}_{\mathbb{K}}$. For intuition, it is helpful to think that the image of the subgroup $\mathbb{K}^{\perp}$ in $\mathfrak{T}$ is the subgroup onto which the corresponding abelian (or surface) group retracts and so $\mathbb{G}_{\mathbb{K}} \perp$ is the directly indecomposable canonical parabolic subgroup where the abelian (or surface) subgroup is mapped by the discriminating family.

Definition 5.2 To any graph tower $\mathfrak{T}$, we associate a partially commutative group $\mathbb{H}$ and an epimorphism $\pi: \mathbb{H} \rightarrow \mathfrak{T}$. The partially commutative group $\mathbb{H}$ is defined via its commutation graph $\Gamma$. The set of edges $E(\Gamma)$ of the graph $\Gamma$ is subdivided into two disjoints sets, the set of $d$-edges and the set of $c$-edges, ie $E(\Gamma)=E_{d}(\Gamma) \cup E_{c}(\Gamma)$, $E_{d}(\Gamma) \cap E_{c}(\Gamma)=\varnothing$. Furthermore, the set of edges $E_{c}(\Gamma)$ satisfies

$$
\text { if }(x, y),(y, z) \in E_{c}(\Gamma) \text {, then }(x, z) \in E_{c}(\Gamma) \text {. }
$$

For every subgroup $\mathbb{K}$ of $\mathbb{H}$, abusing the notation, we denote the image $\pi(\mathbb{K})$ of $\mathbb{K}$ in $\mathfrak{T}$ by $\mathbb{K}$.

We define a $\mathbb{G}$-graph tower as an iterated sequence. We denote graph towers by $\mathfrak{T}$ and write $\mathfrak{T}^{l}$, to indicate that the graph tower $\mathfrak{T}^{l}$ is of height $l$.

A $\mathbb{G}$-graph tower $\mathfrak{T}^{0}$ of height 0 is our fixed partially commutative group $\mathbb{G}$. In this case, the partially commutative group associated to $\mathfrak{T}^{0}$ is also $\mathbb{G}=\mathbb{H}\left(\Gamma_{0}\right)$, all edges of $\Gamma_{0}$ are $d$-edges and the epimorphism $\pi_{0}$ is the identity.

Assume that $\mathfrak{T}^{l-1}$ is a $\mathbb{G}$-graph tower of height $l-1, \mathbb{H}\left(\Gamma_{l-1}\right)$ is its associated partially commutative group and $\mathfrak{T}^{l-1}=\mathbb{H}\left(\Gamma_{l-1}\right) / \mathrm{ncl}\left\langle S_{l-1}\right\rangle$. A $\mathbb{G}$-graph tower $\mathfrak{T}^{l}$ 
of height $l$, the associated partially commutative group $\mathbb{H}\left(\Gamma_{l}\right)$ and the epimorphism $\pi_{l}: \mathbb{H}\left(\Gamma_{l}\right) \rightarrow \mathfrak{T}^{l}$ are constructed using one of the following alternatives.

Basic floor The graph $\Gamma_{l}$ is defined as follows:

- $V\left(\Gamma_{l}\right)=V\left(\Gamma_{l-1}\right) \cup\left\{x_{1}^{l}, \ldots, x_{m_{l}}^{l}\right\}$.

- $E_{d}\left(\Gamma_{l}\right)=E_{d}\left(\Gamma_{l-1}\right) \cup\left\{\left(x_{i}^{l}, x^{l-1}\right) \mid i=1, \ldots, m_{l}, x^{l-1}=\operatorname{alph}(\mathbb{K})\right\}$, where $\mathbb{K}$ is an $E_{d}\left(\Gamma_{l-1}\right)$-coirreducible subgroup of $\mathbb{H}\left(\Gamma_{l-1}\right)$.

- $E_{c}\left(\Gamma_{l}\right)=E_{c}\left(\Gamma_{l-1}\right)$ if the subgroup $\mathbb{K}^{\perp}$ is directly indecomposable (as a subgroup of $\left.\mathbb{H}\left(\Gamma_{l-1}\right)\right)$.

- $E_{c}\left(\Gamma_{l}\right)=E_{c}\left(\Gamma_{l-1}\right) \cup\left\{\left(x_{i}^{l}, x_{j}^{l}\right) \mid 1 \leq i<j \leq m_{l}\right\} \cup\left\{\left(x_{i}^{l}, x^{l-1}\right) \mid 1 \leq i \leq\right.$ $\left.m, x^{l-1}=\operatorname{alph}\left(\mathbb{K}^{\perp}\right)\right\}$ if the subgroup $\mathbb{K}^{\perp}$ is directly decomposable (we will show in Lemma 6.2, that, in this case, $\mathbb{K}^{\perp}$ is $E_{c}\left(\Gamma_{l-1}\right)$-abelian).

We set $\mathbb{H}\left(\Gamma_{l}\right)$ to be the associated partially commutative group. It follows from the definition that $\mathbb{H}\left(\Gamma_{l-1}\right)$ is a retraction of $\mathbb{H}\left(\Gamma_{l}\right)$ and so is $\mathfrak{T}_{l-1}$ of $\mathbb{H}\left(\Gamma_{l}\right) / \operatorname{ncl}\left\langle S_{l-1}\right\rangle$. The group $\mathfrak{T}^{l}$ is a quotient of $\mathbb{H}\left(\Gamma_{l}\right) / \operatorname{ncl}\left\langle S_{l-1}\right\rangle$ by $\operatorname{ncl}\left\langle S\left(x^{l}, \mathbb{H}\left(\Gamma_{l-1}\right)\right)\right\rangle$, ie $\mathfrak{T}^{l}=$ $\mathbb{H}\left(\Gamma_{l}\right) / \operatorname{ncl}\left\langle S_{l-1}, S\right\rangle$, where the set of relations $S$ is

the set of basic relations $\left[C_{\mathfrak{T}^{l-1}}\left(\mathbb{K}^{\perp}\right), x_{i}^{l}\right]=1,1 \leq i \leq m_{l}$.

Abelian floor The graph $\Gamma_{l}$ is defined as follows:

- $V\left(\Gamma_{l}\right)=V\left(\Gamma_{l-1}\right) \cup\left\{x_{1}^{l}, \ldots, x_{m_{l}}^{l}\right\}$.

- $E_{d}\left(\Gamma_{l}\right)=E_{d}\left(\Gamma_{l-1}\right) \cup\left\{\left(x_{i}^{l}, x^{l-1}\right) \mid i=1, \ldots, m_{l}, x^{l-1}=\operatorname{alph}(\mathbb{K})\right\}$, where $\mathbb{K}$ is an $E_{d}\left(\Gamma_{l-1}\right)$-coirreducible subgroup of $\mathbb{H}\left(\Gamma_{l-1}\right)$.

- $E_{c}\left(\Gamma_{l}\right)=E_{c}\left(\Gamma_{l-1}\right) \cup\left\{\left(x_{i}^{l}, x_{j}^{l}\right) \mid 1 \leq i<j \leq m_{l}\right\}$ if the subgroup $\mathbb{K}^{\perp}$ is directly indecomposable (as a subgroup of $\left.\mathbb{H}\left(\Gamma_{l-1}\right)\right)$.

- $E_{c}\left(\Gamma_{l}\right)=E_{c}\left(\Gamma_{l-1}\right) \cup\left\{\left(x_{i}^{l}, x_{j}^{l}\right) \mid 1 \leq i<j \leq m_{l}\right\} \cup\left\{\left(x_{i}^{l}, x^{l-1}\right) \mid 1 \leq i \leq\right.$ $\left.m, x^{l-1}=\operatorname{alph}\left(\mathbb{K}^{\perp}\right)\right\}$ if the subgroup $\mathbb{K}^{\perp}$ is directly decomposable (we will show in Lemma 6.2, that, in this case, $\mathbb{K}^{\perp}$ is $E_{c}\left(\Gamma_{l-1}\right)$-abelian).

We set $\mathbb{H}\left(\Gamma_{l}\right)$ to be the associated partially commutative group. It follows from the definition that $\mathbb{H}\left(\Gamma_{l-1}\right)$ is a retraction of $\mathbb{H}\left(\Gamma_{l}\right)$ and so is $\mathfrak{T}^{l-1}$ of $\mathbb{H}\left(\Gamma_{l}\right) / \operatorname{ncl}\left\langle S_{l-1}\right\rangle$. The group $\mathfrak{T}^{l}$ is a quotient of $\mathbb{H}\left(\Gamma_{l}\right) / \operatorname{ncl}\left\langle S_{l-1}\right\rangle$ by $\operatorname{ncl}\left\langle S\left(x^{l}, \mathbb{H}\left(\Gamma_{l-1}\right)\right)\right\rangle$, ie $\mathfrak{T}^{l}=$ $\mathbb{H}\left(\Gamma_{l}\right) / \operatorname{ncl}\left\langle S_{l-1}, S\right\rangle$, where the set of relations $S$ is one of the following types:

- The relations $\left[C_{\mathfrak{T}^{l-1}}(u), x_{i}^{l}\right]=1$, where $u \in \mathbb{K}^{\perp}<\mathbb{H}\left(\Gamma_{l-1}\right)$ is a nontrivial cyclically reduced root block element.

- The set of basic relations. 
Quadratic floor The graph $\Gamma_{l}$ is defined as follows:

- $V\left(\Gamma_{l}\right)=V\left(\Gamma_{l-1}\right) \cup\left\{x_{1}^{l}, \ldots, x_{m_{l}}^{l}\right\}$.

- $E_{d}\left(\Gamma_{l}\right)=E_{d}\left(\Gamma_{l-1}\right) \cup\left\{\left(x_{i}^{l}, x^{l-1}\right) \mid i=1, \ldots, m_{l}, x^{l-1}=\operatorname{alph}(\mathbb{K})\right\}$, where $\mathbb{K}$ is a $E_{d}\left(\Gamma_{l-1}\right)$-coirreducible subgroup of $\mathbb{H}\left(\Gamma_{l-1}\right)$.

- $E_{c}\left(\Gamma_{l}\right)=E_{c}\left(\Gamma_{l-1}\right)$ if the subgroup $\mathbb{K}^{\perp}$ is directly indecomposable (as a subgroup of $\left.\mathbb{H}\left(\Gamma_{l-1}\right)\right)$.

- $E_{c}\left(\Gamma_{l}\right)=E_{c}\left(\Gamma_{l-1}\right) \cup\left\{\left(x_{i}^{l}, x_{j}^{l}\right) \mid 1 \leq i<j \leq m_{l}\right\} \cup\left\{\left(x_{i}^{l}, x^{l-1}\right) \mid 1 \leq i \leq\right.$ $\left.m, x^{l-1}=\operatorname{alph}\left(\mathbb{K}^{\perp}\right)\right\}$ if the subgroup $\mathbb{K}^{\perp}$ is directly decomposable (we will see in Lemma 6.2, that, in this case, $\mathbb{K}^{\perp}$ is $E_{c}\left(\Gamma_{l-1}\right)$-abelian).

We set $\mathbb{H}\left(\Gamma_{l}\right)$ to be the associated partially commutative group. It follows from the definition that $\mathbb{H}\left(\Gamma_{l-1}\right)$ is a retraction of $\mathbb{H}\left(\Gamma_{l}\right)$ and so is $\mathfrak{T}^{l-1}$ of $\mathbb{H}\left(\Gamma_{l}\right) / \operatorname{ncl}\left\langle S_{l-1}\right\rangle$. The group $\mathfrak{T}^{l}$ is a quotient of $\mathbb{H}\left(\Gamma_{l}\right) / \operatorname{ncl}\left\langle S_{l-1}\right\rangle$ by $\operatorname{ncl}\left\langle S\left(x^{l}, \mathbb{H}\left(\Gamma_{l-1}\right\rangle\right)\right)$, ie $\mathfrak{T}^{l}=$ $\mathbb{H}\left(\Gamma_{l}\right) / \operatorname{ncl}\left\langle S_{l-1}, S\right\rangle$, where the set of relations $S$ consists of the set of basic relations $\left\{\left[C_{\mathfrak{T}^{l-1}}\left(\mathbb{K}^{\perp}\right), x_{i}^{l}\right]=1 \mid 1 \leq i \leq m_{l}\right\}$ and a relation $W$ of one of the two following forms: (orientable)

$$
\begin{aligned}
& {\left[x_{1}, x_{2}\right] \cdots\left[x_{2 g-1}, x_{2 g}\right] u_{2 g+1}{ }^{x_{2 g+1}} \cdots u_{m}{ }^{x_{m}}} \\
& \quad=\left[v_{1}, v_{2}\right] \cdots\left[v_{2 g-1}, v_{2 g}\right] u_{2 g+1} w_{2 g+1} \cdots u_{m}{ }^{w_{m}}
\end{aligned}
$$

or (nonorientable)

$$
x_{1}^{2} \cdots x_{2 g}^{2} u_{2 g+1} x_{2 g+1} \cdots u_{m}{ }^{x_{m}}=v_{1}^{2} \cdots v_{2 g}^{2} u_{2 g+1} w_{2 g+1} \cdots u_{m}{ }^{w_{m}},
$$

where $u_{i}, v_{j}, w_{k} \in \mathbb{K}^{\perp}$ and either

$\circledast$ the Euler characteristic of $W$ is at most -2 , or $W$ corresponds to a punctured torus and the subgroup $\left\langle u_{i}, v_{j}, w_{k} \mid i, k=2 g+1, \ldots, m, j=1, \ldots, 2 g,\right\rangle$ of $\mathfrak{T}^{l-1}$ is nonabelian, ie the retraction of the (punctured) surface onto $\mathfrak{T}^{l-1}$ is nonabelian; or

$\circledast \quad g+m \geq 2$ and

- the subgroup $\left\langle\left[v_{1}, v_{2}\right], \ldots,\left[v_{2 g-1}, v_{2 g}\right], u_{2 g+1} w_{2 g+1}, \ldots, u_{m}{ }^{w_{m}}\right\rangle$ is nonabelian, where $W$ is orientable or

- the subgroup $\left\langle v_{1}^{2}, \ldots, v_{2 g}^{2}, u_{2 g+1}{ }^{w_{2 g+1}}, \ldots, u_{m}{ }^{w_{m}}\right\rangle$ is nonabelian, where $W$ is nonorientable,

that is, the solution is not atom-commutative (see Kharlampovich and Myasnikov [37, Definition 11]). 
Lemma 5.3 The graph tower $\mathfrak{T}^{l}$ admits one of the following decompositions:

(a1) $\mathfrak{T}^{l-1} *_{\mathfrak{T}^{l-1}}\left(\mathbb{K}^{\perp}\right)\left(C_{\mathfrak{T}^{l-1}}\left(\mathbb{K}^{\perp}\right) \times\left\langle x_{1}^{l}, \ldots, x_{m_{l}}^{l}\right\rangle\right.$ ) (if $S$ is basic and $\mathbb{K}^{\perp}$ is nonabelian).

(a2) $\mathfrak{T}^{l-1} *_{C_{\mathfrak{\tau} l-1}\left(\mathbb{K}^{\perp}\right)}\left(C_{\mathfrak{T}^{l-1}}\left(\mathbb{K}^{\perp}\right) \times\left\langle x_{1}^{l}, \ldots, x_{m_{l}}^{l} \mid\left[x_{i}^{l}, x_{j}^{l}\right]=1,1 \leq i, j \leq m_{l}, i \neq j\right\rangle\right)$ (if $S$ is basic and $\mathbb{K}^{\perp}$ is abelian).

(b1) $\mathfrak{T}^{l-1} *_{\mathfrak{T}^{l-1}(u)}\left(C_{\mathfrak{T}^{l-1}}(u) \times\left\langle x_{1}^{l}, \ldots, x_{m_{l}}^{l} \mid\left[x_{i}^{l}, x_{j}^{l}\right]=1,1 \leq i, j \leq m_{l}, i \neq j\right\rangle\right)$ (if $S$ is abelian and $u$ is nontrivial).

(b2) $\mathfrak{T}^{l-1} *_{\mathfrak{T}^{l-1}\left(\mathbb{K}^{\perp}\right)}\left(C_{\mathfrak{T}^{l-1}}\left(\mathbb{K}^{\perp}\right) \times\left\langle x_{1}^{l}, \ldots, x_{m_{l}}^{l} \mid\left[x_{i}^{l}, x_{j}^{l}\right]=1,1 \leq i, j \leq m_{l}, i \neq j\right\rangle\right)$ (if $S$ is abelian and $\mathbb{K}^{\perp}$ is nonabelian).

(c) $\mathfrak{T}^{l-1} *_{C_{\mathfrak{T}} l-1}\left(\mathbb{K}^{\perp}\right) \times\left\langle u_{2 g+1}, \ldots, u_{m}\right\rangle\left(\left\langle u_{2 g+1}, \ldots, u_{m}, x_{1}^{l}, \ldots, x_{m_{l}}^{l} \mid W\right\rangle \times C_{\mathfrak{T}^{l-1}}\left(\mathbb{K}^{\perp}\right)\right)$ (if $W$ satisfies one of the properties $\circledast$ and $\circledast \circledast$ from Definition 5.2).

Proof The only decomposition that does not follow immediately from the definition of a graph tower is c). From the definition, we have that

$$
\begin{array}{r}
\mathfrak{T}^{l-1} *\left\langle C_{\mathfrak{T}^{l-1}}\left(\mathbb{K}^{\perp}\right), u_{2 g+1}, \ldots, u_{m}\right\rangle\left(\left\langle C_{\mathfrak{T}^{l-1}}\left(\mathbb{K}^{\perp}\right), u_{2 g+1}, \ldots, u_{m}, x^{l}\right| W=1,\right. \\
\left.\left.\left[C_{\mathfrak{T}^{l-1}}\left(\mathbb{K}^{\perp}\right), x^{l}\right]=1\right\rangle\right),
\end{array}
$$

where $x^{l}=\left\{x_{1}^{l}, \ldots, x_{m_{l}}^{l}\right\}$. In Section 7, we prove the subgroup

$$
\left\langle C_{\mathfrak{T}^{l-1}}\left(\mathbb{K}^{\perp}\right), u_{2 g+1}, \ldots, u_{m}\right\rangle
$$

is the direct product of $C_{\mathfrak{T}^{l-1}}\left(\mathbb{K}^{\perp}\right)$ and $\left\langle u_{2 g+1}, \ldots, u_{m}\right\rangle$, hence decomposition (c).

Remark 5.4 - Since graph towers can be described as iterated sequences of amalgamated products and HNN-extensions, each graph tower $\mathfrak{T}^{i}$ naturally embeds into the graph tower $\mathfrak{T}^{i+1}$. In fact, for each floor, there is a natural retraction of $\mathfrak{T}^{i+1}$ onto $\mathfrak{T}^{i}$.

- If the graph tower is constructed using only basic floors, then $\mathfrak{T}$ and $\mathbb{H}$ coincide, ie the graph tower is itself a partially commutative group.

- Notice that graph towers are a natural generalisation of the notions of $\omega-$ residually free towers and NTQ-groups. Indeed, let $\mathbb{G}$ be a free group and assume by induction that $\mathfrak{T}^{l-1}$ is an NTQ-group.

By assumption, in cases (a1), (b2) and (c) the group $\mathbb{K}^{\perp}$ is nonabelian. In particular, since limit groups have the CSA property, it follows that the centraliser $C_{\mathfrak{T}^{l-1}}\left(\mathbb{K}^{\perp}\right)$ is trivial. Therefore, case (a1) corresponds to the free product of the group $\mathfrak{T}^{l-1}$ and a free group; case (b2) corresponds to the free product of $\mathfrak{T}^{l-1}$ and a free abelian group; and case (c) corresponds to a (sequence of) 
amalgamated products (and HNN-extensions) of $\mathfrak{T}^{l-1}$ with a surface group (so that the natural retraction of the surface to $\mathfrak{T}^{l-1}$ is nonabelian).

In cases (a2) and (b1), the subgroup $\mathbb{K}^{\perp}$ is abelian. Hence, these cases correspond to extension of centralisers of maximal abelian subgroups, or equivalently, to an amalgamated product of $\mathfrak{T}^{l-1}$ and a free abelian group (amalgamated by a maximal (in $\mathfrak{T}^{l-1}$ ) abelian subgroup).

We therefore obtain an NTQ-group. Notice that the difference with an $\omega-$ residually free tower is that our construction is not canonical in the following sense. In the construction of an $\omega$-residually free tower, at a given floor one attaches all the pieces corresponding to the abelian JSJ-decomposition. In our case, pieces are attached one by one, hence the same graph tower can be constructed in several different ways (for example, at the same level one can first attach an abelian group and then a surface group or vice versa).

- Notice that, in general, the centraliser of a set of elements in a partially commutative group is neither commutative transitive, nor malnormal, nor small (see [22]). Hence, the splittings we find do not correspond to the (abelian) JSJ decomposition. Nevertheless, the decomposition we find plays the role analogous to the one the JSJ plays for limit groups: one can define modular automorphisms and show that under some restrictions on the decomposition (similar to the ones for constructible limit groups), the groups we consider are discriminated using the retraction and appropriate modular automorphisms.

- As we mentioned above, the construction of the graph tower is not canonical. In the case of free groups, Kharlampovich and Miasnikov [39] described an elimination process based on the Makanin-Razborov process that finds the (cyclic) JSJ decomposition of a fully residually free group. If an appropriate JSJ theory is developed for the class of groups we deal with in this paper, then one could modify the process developed in [14] to obtain a canonical construction of the graph tower.

\section{Graph tower associated to a branch of the tribal Makanin- Razborov diagram}

The goal of this section is, given a branch $\Omega_{0} \rightarrow \cdots \rightarrow \Omega_{q}$ of the tribal solution tree, to construct a graph tower $\mathfrak{T}_{0}$ as well as a homomorphism $\tau_{0}$ from $G_{\Omega_{0}}$ to $\mathfrak{T}_{0}$ so that for every homomorphism $H$ from $G_{R\left(\Omega_{0}\right)}$ to $\mathbb{G}$ that factors through the branch there exists a homomorphism $H^{\prime}$ from $\mathfrak{T}_{0}$ to $\mathbb{G}$ such that the following diagram is 


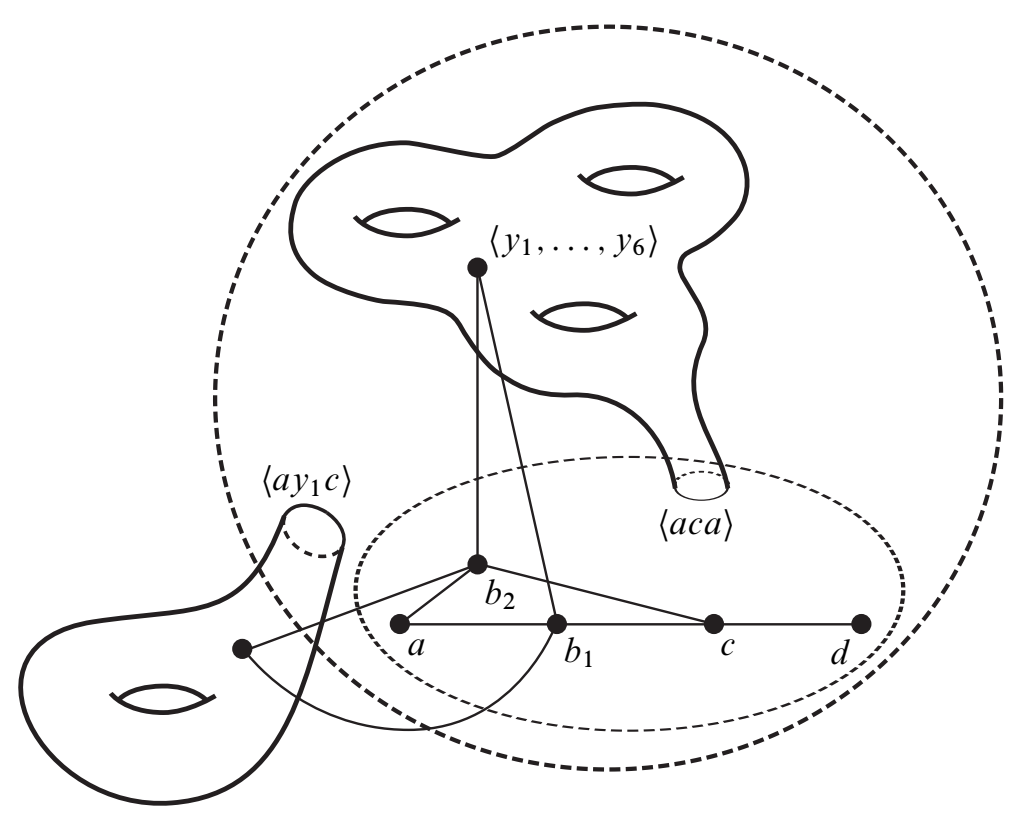

Figure 2: Graph tower of height 2: edges of the graph correspond to commutation of the generators.

commutative:

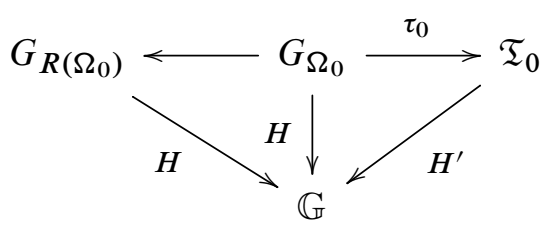

The graph tower is constructed using induction on the height of the branch. One begins with the group associated to the leaf of the branch, which is a partially commutative group discriminated by $\mathbb{G}$ and keeps building the graph tower according to the type of epimorphism associated to the edges (or more precisely, the types of automorphism groups associated to the vertices) of the branch.

Our induction hypothesis at step $q$ is as follows:

(IH) Given a generalised equation $\Omega_{q}$, there exists a graph tower $\left(\mathfrak{T}_{q}, \mathbb{H}_{q}\right)$, where $\mathbb{H}_{q}=\mathbb{H}\left(\Gamma_{q}\right)$, and a homomorphism $\tau_{q}$ from $G_{\Omega_{q}}$ to $\mathfrak{T}_{q}$ such that for every solution $H^{(q)}$ of the fundamental sequence, there exists a homomorphism 
$H^{(q)}: \mathfrak{T}_{q} \rightarrow \mathbb{G}$ that makes the diagram

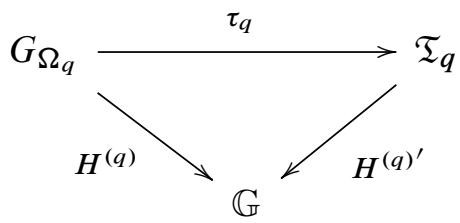

commutative; notice that if $\pi_{q}: \mathbb{H}_{q} \rightarrow \mathfrak{T}_{q}$, then $\pi_{q} H^{(q)^{\prime}}$ is a homomorphism from $\mathbb{H}_{q}$ to $\mathbb{G}$ that, abusing the notation, we also denote by $H^{(q)^{\prime}}$.

(IH1) for all generators $x_{i}, x_{j} \in \mathbb{H}_{q}$, we have that $\left(x_{i}, x_{j}\right) \in E_{d}\left(\Gamma_{q}\right)$ if and only if $H^{(q)^{\prime}}\left(x_{i}\right) \leftrightarrows H^{(q)^{\prime}}\left(x_{j}\right)$ for all homomorphisms $H^{(q)^{\prime}}$ induced by a solution $H^{(q)}$ that factors through the branch; furthermore, we have that if $\left(x_{i}, x_{j}\right) \in E_{c}\left(\Gamma_{q}\right)$ then $H^{(q)^{\prime}}\left(x_{i}\right), H^{(q)^{\prime}}\left(x_{j}\right)$ belong to a cyclic subgroup for all homomorphisms $H^{(q) \prime}$ induced by a solution $H^{(q)}$ that factors through the branch;

(IH2) for any item $h_{i}^{(q)}$, if $\tau_{q}\left(h_{i}^{(q)}\right)=y_{i 1}^{(q)} \cdots y_{i k}^{(q)}$, where $y_{i j}^{(q)} \in y^{(q)}$ and $\mathfrak{T}_{q}=$ $\left\langle y^{(q)}\right\rangle$, then

$$
\mathbb{A}\left(H^{(q)^{\prime}}\left(y_{i j}\right)\right) \supset \mathbb{A}\left(H_{i}^{(q)}\right) \text { and } \bigcap_{j=1}^{k} \mathbb{A}\left(H^{(q)^{\prime}}\left(y_{i j}\right)\right)=\mathbb{A}\left(H_{i}^{(q)}\right)
$$

for all solutions $H^{(q)}$ that factor through the branch and homomorphisms $H^{(q)^{\prime}}$ induced by $H^{(q)}$.

The induction hypotheses (IH1) and (IH2) are essential to keep control on the "type" of subgroups whose centralisers we extend. In turn, this is crucial in order to prove that graph towers are discriminated by $\mathbb{G}$.

Remark 6.1 We record the following facts that will be used in the course of the proof.

(1) Groups corresponding to the leaves of $T_{\mathrm{sol}}(\Omega)$ are explicitly described (see eg [14, Proposition 9.1]).

(2) Without loss of generality, one can assume that the word $H(\sigma)$, where $\sigma$ is a closed section of $\Omega$ and $H$ is a solution of the constrained generalised equation $\Omega$ is a subword of a word in the DM-normal form (see [14, Section 3.3.1]).

(3) Automorphisms associated to the vertices of the tree $T_{\text {sol }}(\Omega)$ are completely induced (see [14, Definition 7.2 in Section 7.1]). In short, an automorphism is completely induced if, on the one hand it is tame (induced by an automorphism of the free group) and, on the other hand, it also induces an automorphism of the associated coordinate group over the free group (the coordinate group whose defining relations are the equations, but not the commutation constraints). 
Before turning our attention to the construction of the graph tower, we clarify the structure of orthogonal complements $\mathbb{K}^{\perp}$ of coirreducible subgroups and justify the somewhat nonintuitive definition. The structure of $\mathbb{K}^{\perp}$ allows us, a posteriori, to establish that a discriminating family of the graph tower maps a coirreducible subgroup $\mathbb{K}$ into a closed subgroup of $\mathbb{G}$ and the subgroup $\mathbb{K}^{\perp}$ into a directly indecomposable subgroup (which we treat differently depending on if it is cyclic or nonabelian).

\subsection{Coirreducible subgroups}

The definition of coirreducible subgroup might seem somewhat artificial since we ask that the subgroup $\mathbb{K}^{\perp}$ be $E_{d}(\Gamma)$-directly indecomposable. In the following lemma we clarify the structure of the subgroup $\mathbb{K}^{\perp}$ in the group $\mathbb{H}$.

Lemma 6.2 Let $(\mathfrak{T}, \mathbb{H})$ be a graph tower that satisfies the induction hypotheses $(I H)$ and (IH1). Then, if $\mathbb{K}^{\perp}$ is an $E_{d}(\Gamma)$-directly indecomposable canonical parabolic subgroup of $\mathbb{H}$, then $\mathbb{K}^{\perp}$ is either a directly indecomposable subgroup of $\mathbb{H}$ or $E_{c}(\Gamma)$ abelian.

Proof Let $\mathbb{H}=\mathbb{G}(\Gamma)$. If follows from the induction hypothesis (IH1) that if $(x, y) \in$ $E_{c}(\Gamma)$, then $(x, z) \in E_{d}(\Gamma)$ if and only if $(y, z) \in E_{d}(\Gamma)$.

Assume that $\mathbb{K}^{\perp}$ is directly decomposable, but $E_{d}(\Gamma)$-directly indecomposable in $\mathbb{H}$. Without loss of generality, assume that $\mathbb{K}^{\perp}=\mathbb{K}_{1} \times \mathbb{K}_{2}$. We use induction on the rank of $\mathbb{K}_{2}$ to prove that then $\mathbb{K}^{\perp}$ is $E_{c}(\Gamma)$-abelian.

Suppose that $\mathbb{K}_{2}$ has rank 1 , ie $\mathbb{K}_{2}=\langle x\rangle$. Let $\operatorname{alph}\left(\mathbb{K}_{1}\right)=\operatorname{alph}\left(\mathbb{K}_{1, c}\right) \cup \operatorname{alph}\left(\mathbb{K}_{1, d}\right)$ where $\operatorname{alph}\left(\mathbb{K}_{1, c}\right)=\left\{y \in \operatorname{alph}\left(\mathbb{K}_{1}\right) \mid(x, y) \in E_{c}(\Gamma)\right\}$ and $\operatorname{alph}\left(\mathbb{K}_{1, d}\right)=\left\{y \in \operatorname{alph}\left(\mathbb{K}_{1}\right) \mid\right.$ $\left.(x, y) \in E_{d}(\Gamma)\right\}$. Since $\mathbb{K}^{\perp}$ is $E_{d}(\Gamma)$-directly indecomposable, we have $\mathbb{K}_{1} \neq \mathbb{K}_{1, d}$. If $\mathbb{K}_{1}=\mathbb{K}_{1, c}$, then since the set $E_{c}(\Gamma)$ satisfies condition (8), it follows that $\mathbb{K}^{\perp}$ is $E_{c}(\Gamma)$-abelian. Otherwise, we have that $\left(x, k_{d}\right) \in E_{d}(\Gamma)$, for all $k_{d} \in \mathbb{K}_{1, d}$ and $\left(x, k_{c}\right) \in E_{c}(\Gamma)$ for all $k_{c} \in \mathbb{K}_{1, c}$. It follows from the above observations that $\left(k_{d}, k_{c}\right) \in$ $E_{d}(\Gamma)$ for all $k_{d} \in \mathbb{K}_{1, d}$ and for all $k_{c} \in \mathbb{K}_{1, c}$ and hence $\mathbb{K}^{\perp}=\mathbb{K}_{1, d} \times\left\langle\mathbb{K}_{1, c}, x\right\rangle$ is $E_{d}(\Gamma)$-directly decomposable, a contradiction.

Assume that $\mathbb{K}_{2}$ has rank $r$. Let $x \in \mathbb{K}_{1}$ and let alph $\left(\mathbb{K}_{2}\right)=\operatorname{alph}\left(\mathbb{K}_{2, c}\right) \cup \operatorname{alph}\left(\mathbb{K}_{2, d}\right)$, where $\operatorname{alph}\left(\mathbb{K}_{2, c}\right)=\left\{y \in \operatorname{alph}\left(\mathbb{K}_{2}\right) \mid(x, y) \in E_{c}(\Gamma)\right\}$ and $\operatorname{alph}\left(\mathbb{K}_{2, d}\right)=\left\{y \in \operatorname{alph}\left(\mathbb{K}_{2}\right) \mid\right.$ $\left.(x, y) \in E_{d}(\Gamma)\right\}$. Notice that since by assumption $\mathbb{K}^{\perp}$ is $E_{d}(\Gamma)$-directly indecomposable, we have that the set $\operatorname{alph}\left(\mathbb{K}_{2}, c\right)$ is nonempty. As above, it follows that $\mathbb{K}^{\perp}=\left\langle\mathbb{K}_{1} \cup \mathbb{K}_{2, c}\right\rangle \times \mathbb{K}_{2, d}$ and the rank of $\mathbb{K}_{2, d}$ is strictly less than the rank of $\mathbb{K}_{2}$. By induction we conclude that $\mathbb{K}^{\perp}$ is $E_{c}(\Gamma)$-abelian. 


\subsection{Base of induction}

If the height $q$ of the branch equals 0 , then the group $G_{\Omega_{0}}$ is the group associated to the leaf of the tree $T_{\mathrm{sol}}(G)$. By the description of the leaves of the tree $T_{\text {sol }}$ (see [14, Proposition 9.1] and its proof), it follows that $G_{\Omega_{0}}$ is a partially commutative group, which is fully residually $\mathbb{G}$ and it is built from $\mathbb{G}$ by a sequence of extensions of centralisers of directly indecomposable canonical parabolic subgroups. Hence, in our terms, this partially commutative group is a graph tower over $\mathbb{G}$, where each floor is basic, and at each floor $\mathfrak{T}_{k}=\mathbb{H}_{k}$ (and $\tau_{k}$ is the identity).

If the variables $x_{1}, \ldots, x_{l_{k}}$ extend a centraliser of a cyclic canonical parabolic subgroup $\left\langle a_{i}\right\rangle$ in $\mathbb{H}\left(\Gamma_{k-1}\right)$, then the subgroup $\left\langle a_{i}, x_{1}, \ldots, x_{l_{k}}\right\rangle$ in $\mathbb{H}\left(\Gamma_{k}\right)$ is free abelian and, by definition, the edges $\left(a_{i}, x_{i}\right),\left(x_{i}, x_{j}\right)$ belong to $E_{c}\left(\Gamma_{k}\right)$. All the other (new) edges belong to $E_{d}\left(\Gamma_{k}\right)$.

From the construction of the leaves of the tree $T_{\text {sol }}(G)$ (see [14, Proposition 9.1]) we obtain that the graph tower satisfies the induction hypothesis:

- The graph tower $\left(\mathfrak{T}=\mathbb{H}_{q}, \mathbb{H}_{q}\right)$ trivially makes diagram (11) commutative.

- By definition of a solution of a generalised equation and since $H^{\prime}=H$, for all $x_{i}, x_{j} \in \mathbb{H}_{q}$, we have that $\left(x_{i}^{(1)}, x_{j}^{(1)}\right) \in E_{d}\left(\Gamma_{q}\right)$ if and only if $H^{\prime}\left(x_{i}\right) \leftrightarrows H^{\prime}\left(x_{j}\right)$ for every homomorphism $H^{\prime}$ induced by a solution $H$ that factors through the branch. Furthermore, if $\left(x_{i}, x_{j}\right) \in E_{c}\left(\Gamma_{q}\right)$, then $H^{\prime}\left(x_{i}\right), H^{\prime}\left(x_{j}\right)$ belong to the same cyclic subgroup for every homomorphism $H^{\prime}$ induced by a solution $H$ that factors through the branch.

- Since $\tau_{q}: G_{\Omega_{q}} \rightarrow \mathbb{H}_{q}$ is the identity, it obviously satisfies the hypothesis (IH2): if $\tau_{q}\left(h_{i}\right)=y_{i 1} \cdots y_{i k}$, then $\mathbb{A}\left(H^{\prime}\left(y_{i j}\right)\right) \supset \mathbb{A}\left(H_{i}\right)$ and $\bigcap_{j=1}^{k} \mathbb{A}\left(H^{\prime}\left(y_{i j}\right)\right)=\mathbb{A}\left(H_{i}\right)$ for all solutions $H$ that factor through the branch and homomorphisms $H^{\prime}$ induced by $H$.

\subsection{Step of induction}

Suppose that the graph tower $\mathfrak{T}_{1}$ exists and the induction hypotheses (IH), (IH1) and (IH2) are satisfied for the group $G_{\Omega_{1}}$. We show how to construct the graph tower $\mathfrak{T}_{0}$ for the group $G_{\Omega_{0}}$ and prove that the graph tower satisfies all the induction hypothesis.

We construct the group $\mathfrak{T}_{0}$ starting from the graph tower $\mathfrak{T}_{1}$. The construction of $\mathfrak{T}_{0}$ depends on the type of the vertex $v_{0}$. It follows by construction of the tribal solution tree $T_{\text {sol }}(\Omega)$ that one of the following hold:

- The automorphism group associated to $\Omega_{0}$ is trivial; in this case every solution of $\Omega_{0}$ that factors through the branch also factors through the group $G_{\Omega_{1}}$. 
- The automorphism group associated to $\Omega_{0}$ is nontrivial. Then one of the following hold:

(1) $\Omega_{0}$ is a tribal general equation of linear type; in this case, every solution that factors through the branch is the composition of an automorphism from the automorphism group associated to $\Omega_{0}$ (ie automorphisms invariant with respect to the kernel), the epimorphism $\pi\left(v_{0}, v_{1}\right)$ and a homomorphism from $G_{R\left(\Omega_{1}\right)}$ to $\mathbb{G}$ (that factors through the branch).

(2) $\Omega_{0}$ is tribal of type 12 ; in this case, every solution that factors through the branch is the composition of an automorphism from the automorphism group associated to $\Omega_{0}$ (ie automorphisms invariant with respect to the nonquadratic part), the epimorphism $\pi\left(v_{0}, v_{1}\right)$ and a homomorphism from $G_{R\left(\Omega_{1}\right)}$ to $\mathbb{G}$ (that factors through the branch).

(3) $\Omega_{0}$ is tribal of type 15 ; then every solution that factors through the branch is the composition of an automorphism from the group of automorphisms generated by automorphisms invariant with respect to the nonquadratic part and automorphisms associated to regular periodic structures, the epimorphism $\pi\left(v_{0}, v_{1}\right)$ and a homomorphism from $G_{R\left(\Omega_{1}\right)}$ to $\mathbb{G}$ (that factors through the branch).

(4) $\Omega_{0}$ is of type 2 ; then all solutions factor through a generalised equation which is singular (or strongly singular) with respect to a periodic structure and so, in this case, every solution is a composition of an automorphism associated to a vertex of type 2 , the epimorphism $\pi\left(v_{0}, v_{1}\right)$ and a homomorphism from $G_{R\left(\Omega_{1}\right)}$ to $\mathbb{G}$ (that factors through the branch).

\subsection{Trivial group of automorphisms}

If the group of automorphisms associated to a vertex of the tree $T_{\text {sol }}$ is trivial, we set $\mathfrak{T}_{0}=\mathfrak{T}_{1}, \mathbb{H}_{0}=\mathbb{H}_{1}$ and $\tau_{0}$ to be $\pi\left(v_{0}, v_{1}\right) \tau_{1}$, where $\tau_{1}$ is the homomorphism from $G_{\Omega_{1}}$ to $\mathfrak{T}_{1}$ that makes diagram (11) commutative. If the group of automorphisms is trivial, any homomorphism that factors through the branch also factors through $G_{\Omega_{1}}$ and so $\tau_{0}$ and $\mathfrak{T}_{0}$ make diagram (11) commutative.

\subsection{Quadratic case}

By replacing $\Omega_{0}$ by $\Omega_{0}^{\prime}$ if necessary (see diagram (7) and preceding discussion), we can assume that short bases in $\Omega_{0}$ that belong to tribes that strictly dominate the minimal tribe are quadratic-coefficient (see Remark 4.5 and discussion above). 
We aim to prove that there exists the following commutative diagram

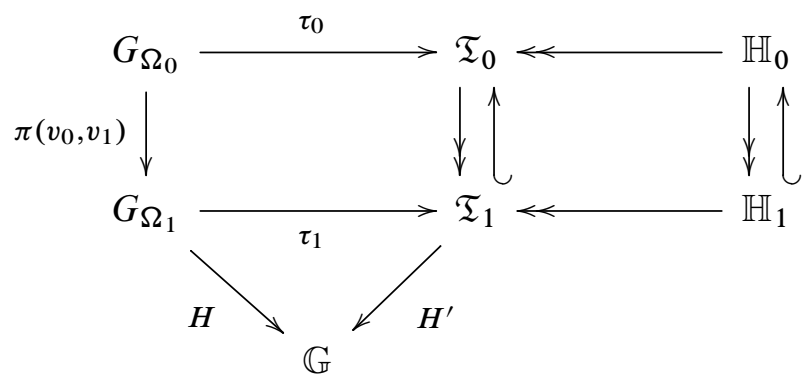

and that the graph tower $\left(\mathfrak{T}_{0}, \mathbb{H}_{0}\right)$ satisfies all the induction hypotheses. We fix the following notation for the generators of the groups we consider: $G_{\Omega_{i}}=\left\langle h^{(i)}\right\rangle, i=0,1$, $\mathbb{H}_{1}=\left\langle x^{(1)}\right\rangle$, and $\mathfrak{T}_{1}=\left\langle y^{(1)}\right\rangle$. Denote by $\varphi_{0}$ the composition of $\pi\left(v_{0}, v_{1}\right)$ and $\tau_{1}$.

Let $\mathcal{N}$ be the subset of the set of quadratic bases of $\Omega_{0}$ so that, for each pair of dual bases $\mu, \Delta(\mu)$, the set $\mathcal{N}$ contains exactly one of them. Let $n$ be the cardinality of the set $\mathcal{N}$. Notice that, by definition, the set $\mathcal{N}$ contains long bases and short bases that belong to the minimal tribe.

For every $\eta \in \mathcal{N}$, set $\varphi_{0}\left(h^{(0)}(\eta)\right)=w_{\eta}\left(y^{(1)}\right)$. We define the canonical parabolic subgroup $\mathbb{K}$ of $\mathbb{H}_{1}$ to be $\mathbb{A}_{E_{d}\left(\Gamma_{1}\right)}\left(\left\{w_{\eta}\left(x^{(1)}\right), \eta \in \mathcal{N}\right\}\right)$.

Lemma 6.3 The subgroup $\mathbb{K}$ of $\mathbb{H}_{1}$ is $E_{d}\left(\Gamma_{1}\right)$-coirreducible.

Proof Let us begin with an observation. Since items of the generalised equation $\Omega_{0}$ have associated parabolic subgroups, there exists a canonical parabolic subgroup $\mathbb{G}_{\mathbb{K}}<\mathbb{G}$ such that for solutions of $\Omega_{0}$, we have that $\left\langle\operatorname{alph}\left(H^{(0)^{\prime}}(\mathbb{K})\right)\right\rangle=\mathbb{G}_{\mathbb{K}}$. By definition of $\mathbb{K}$ and by the induction hypothesis (IH1), we have $H^{(1)^{\prime}}\left(\varphi_{0}(h(\eta))\right) \leftrightarrows \mathbb{G}_{\mathbb{K}}$ for solutions of $\Omega_{1}$. Furthermore, if $a_{i} \leftrightarrows\left\{H^{(1)^{\prime}}\left(\varphi_{0}(h(\eta))\right) \mid \eta \in \mathcal{N}\right\}$, where $a_{i} \in \mathcal{A}$, $\mathbb{G}=\mathbb{G}(\mathcal{A})$, again by the induction hypothesis (IH1), we have that $a_{i} \in \mathbb{K}$ and so $a_{i} \in \mathbb{G}_{\mathbb{K}}$, hence we have that

$$
\left\langle\operatorname{alph}\left(\left\{H^{(1)^{\prime}}\left(\varphi_{0}(h(\eta))\right) \mid \eta \in \mathcal{N}\right\}\right)^{\perp}\right\rangle=\mathbb{G}_{\mathbb{K}} .
$$

Since every solution $H^{(0)}$ that factors through the branch is obtained from a solution $H^{(1)}$ of $\Omega_{1}$ that factors through the branch by precomposing it with a canonical automorphism (and $\pi\left(v_{0}, v_{1}\right)$ ), it follows that $a_{i} \leftrightarrows\left\{H^{(0)}(\eta), \eta \in \mathcal{N}\right\}$ for all solutions $H^{(0)}$ of the branch if and only if $a_{i} \leftrightarrows\left\{H^{(1)}\left(\pi\left(v_{0}, v_{1}\right)(h(\eta))\right), \eta \in \mathcal{N}\right\}$ for all $H^{(1)}$ from the branch, where $a_{i} \in \mathbb{G}$. Therefore $\left\langle\operatorname{alph}\left(\left\{H^{(0)}(\eta), \eta \in \mathcal{N}\right\}\right)^{\perp}\right\rangle=\mathbb{G}_{\mathbb{K}}$ and since all the bases $\eta$ belong to the same minimal tribe, $\left\langle\operatorname{alph}\left(H^{(0)}(\eta)\right)^{\perp}\right\rangle=\mathbb{G}_{\mathbb{K}}$, for all $\eta \in \mathcal{N}$. Furthermore, since any minimal item $h_{i}^{(0)}$ belongs to the same minimal 
tribe, we have that $\left\langle\operatorname{alph}\left(H_{i}^{(0)}\right)^{\perp}\right\rangle=\mathbb{G}_{\mathbb{K}}$. Hence, if $h_{j}^{(0)}$ is so that the tribe of $h_{j}^{(0)}$ dominates the minimal tribe, then we have that $H_{j}^{(0)} \in \mathbb{G}_{\mathbb{K}}^{\perp}$.

Let us now address the statement of the lemma. By definition, the group $\mathbb{K}$ is closed, ie $\mathbb{K}=\mathbb{K}^{\perp \perp}$. We only need to show that $\mathbb{K}^{\perp}$ is $E_{d}\left(\Gamma_{1}\right)$-directly indecomposable.

Assume the contrary, then $\mathbb{K}^{\perp}=\mathbb{K}_{1} \times \cdots \times \mathbb{K}_{r}$, where $r>1$ (and this decomposition is with respect to the edges from $\left.E_{d}\left(\Gamma_{1}\right)\right)$. Without loss of generality, we shall assume that $r=2$. Since items of $\Omega_{0}$ have associated parabolic subgroups, there exist canonical parabolic subgroups $\mathbb{G}_{\mathbb{K}_{i}}<\mathbb{G}, i=1,2$ such that for all solutions $H^{(0)}$ that factor through the branch we have $H^{(0)^{\prime}}\left(\mathbb{K}_{i}\right)=\mathbb{G}_{\mathbb{K}_{i}}, i=1,2$. Furthermore, by the induction hypothesis (IH1), $\mathbb{G}_{\mathbb{K}_{1}} \leftrightarrows \mathbb{G}_{\mathbb{K}_{2}}$. As we have shown, if $h_{i}^{(0)}$ is an item whose tribe dominates the minimal tribe, then $H_{i}^{(0)} \in \mathbb{G}_{\mathbb{K}}^{\perp}<\mathbb{G}_{\mathbb{K}_{1}} \times \mathbb{G}_{\mathbb{K}_{2}}$. It follows by Lemma 3.21, that $H^{(0)}\left(\left[1, \rho_{A}\right]\right) \in \mathbb{G}_{\mathbb{K}_{1}} \times \mathbb{G}_{\mathbb{K}_{2}}$.

By Lemma 3.10, the section $\left[1, \rho_{A}\right]$ is a closed section of $\Omega$ and, by Remark 6.1, the word $H^{(0)}\left[1, \rho_{A}\right]$ is a subword of a word in the DM-normal form. It follows by Lemma 2.3 , that the word $H^{(0)}\left[1, \rho_{A}\right]$ contains only a bounded number of $\mathbb{G}_{\mathbb{K}_{1}} \times \mathbb{G}_{\mathbb{K}_{2}}$ alternations.

In the tribal tree $T(\Omega)$, when applying an entire transformation from $\Omega_{0}$, the only items that could be mapped to a word of length greater than one by the epimorphism $\pi\left(v_{0}, v_{1}\right)$ are $h_{1}^{(0)}$ and $h_{\mathfrak{r}(2)}^{(0)}$, where the boundary connection $\left(2, \mu, n_{2}\right)$ is introduced and $n_{2}$ is introduced between the boundaries $\mathfrak{l}(2)$ and $\mathfrak{l}(2)+1$ of $\Omega_{0}$. The image of $h_{1}^{(0)}$ is a word that contains at least one minimal item, namely the item $h_{\mathrm{r}(2)}^{(1)}$. By Remark 3.19, we have that the image of $h_{\mathrm{l}(2)}^{(0)}$ is the word $h_{\mathrm{r}(2)}^{(1)} h_{n_{2}}^{(1)}$ and both of the items $h_{\mathfrak{l}(2)}^{(1)}, h_{n_{2}}^{(1)}$ are minimal. Hence, the image of $h^{(0)}\left(\left[1, \rho_{A}\right]\right)$ is a word in variables $h^{(1)}$ that contains at least $|h(\mathfrak{M})|+1$ minimal items.

Repeating this argument, we conclude that $\pi\left(v_{0}, v_{k}\right)\left(h^{(0)}\left(\left[1, \rho_{A}\right]\right)\right)$ is a word in $h^{(k)}$ that contains at least $|h(\mathfrak{M})|+k$ minimal items. By [14, Lemma 7.12], in an infinite branch of type 12 , there exists an infinite sequence of generalised equations

$$
\Omega_{0}=\Omega_{n_{1}}=\cdots,
$$

so that every solution $H^{\left(n_{k}\right)}$ of $\Omega_{n_{k}}$ induces a solution $H^{(0)}=\pi\left(v_{0}, v_{n_{k}}\right) H^{\left(n_{k}\right)}$ of $\Omega_{0}$. Therefore, on the one hand, $H^{(0)}\left[1, \rho_{A}\right] \in \mathbb{G}_{\mathbb{K}_{1}} \times \mathbb{G}_{\mathbb{K}_{2}}$. On the other hand, $H^{(0)}\left[1, \rho_{A}\right]$ is graphically equal to a word $w\left(H^{(1)}\right)$ that contains $|h(\mathfrak{M})|+n_{k}$ minimal items. Every minimal item defines at least one $\mathbb{G}_{\mathbb{K}_{1}} \times \mathbb{G}_{\mathbb{K}_{2}}$-alternation. Hence, the word $H^{(0)}\left[1, \rho_{A}\right]$ contains at least $|h(\mathfrak{M})|+n_{k}$ many $\mathbb{G}_{\mathbb{K}_{1}} \times \mathbb{G}_{\mathbb{K}_{2}}$-alternations, deriving a contradiction. Therefore, we have that $s=r=1, H_{i}^{(0)}$ is a block and $\mathbb{K}^{\perp}$ is directly indecomposable. 
Remark 6.4 Our proof of Lemma 6.3 shows that for all solutions $H$ that factor though a given branch, one has that $H_{i} \in \mathbb{G}_{\mathbb{K}} \perp$ is a block element for all minimal items $h_{i}$ of $\Omega$ and $\mathbb{A}\left(H_{i}\right)=\mathbb{A}\left(\mathbb{G}_{\mathbb{K}^{\perp}}\right)$.

Lemma 6.5 Let $\Omega_{0}$ be a tribal generalised equation of type 12. Then there exists an element $U \in G_{\Omega_{0}}$ so that $H(U)$ is irreducible for all solutions $H$ that factor through the branch.

Proof Since the word $H_{1}$ is a block (see Remark 6.4), since the tribe of $h_{i}$ dominates the minimal tribe (see Corollary 3.22), and since the word $H[1, i]$ is reduced as written, it follows that the word $H[1, i]$ is a block element for all $1<i \leq \rho_{A}$. Furthermore, if the word $H[1, i]$ is not irreducible, then it is not cyclically reduced.

If the word $H_{1} H[2, i]=w v w^{-1}$ is not cyclically reduced, then by [24, Proposition 3.18], the word $w=d_{1} d_{2}$, where $d_{1}$ is a left divisor of $H_{1}, H_{1}=d_{1} u_{1}, d_{2}$ is a left divisor of $H[2, i], H[2, i]=d_{2} u_{2}$, and $d_{2} \leftrightarrows u_{1}$. Furthermore, since $H_{1}$ is a block element and $\mathbb{A}\left(d_{2}\right)>\mathbb{A}\left(H_{1}\right)$, it follows that $d_{2} \nleftarrow H_{1}$, hence $d_{1} \neq 1$.

Let $\mu$ be the carrier base of $\Omega$. Suppose $\varepsilon(\mu)=\varepsilon(\Delta(\mu))$. In this case, we show that $H[1, \alpha(\Delta(\mu))-1]$ is irreducible. Assume the contrary. Then, from the above discussion, $H_{1} H[2, \alpha(\Delta(\mu))-1]=d_{1} u d_{1}^{-1}$, where $d_{1} \neq 1$ is a left divisor of $H_{1}$. On the other hand, by Lemma 3.10, the word $H[1, \beta(\Delta(\mu))]=H[1, \alpha(\Delta(\mu))-1] H(\Delta(\mu))$ is reduced, a contradiction since $d_{1}^{-1}$ right-divides $H[1, \alpha(\Delta(\mu))-1]$ and $d_{1}$ leftdivides $H(\Delta(\mu))$.

Suppose now that $\varepsilon(\mu)=-\varepsilon(\Delta(\mu))$. Let $v$ be the (uniquely defined) long base, so that $\alpha(v)=\beta(\mu)-1$. Note that, since $\Omega$ is formally consistent (see [14, Definition 3.9]) we have that $\nu \neq \Delta(\mu)$. Let $\Omega^{\prime}$ be obtained from $\Omega$ by a complete entire transformation. If $\varepsilon(v)=\varepsilon(\Delta(v))$ in $\Omega^{\prime}$, then, by Lemma 3.18, the argument above applies to the generalised equation $\Omega^{\prime}$ and the carrier base $\nu$.

Suppose that $\varepsilon(v)=-\varepsilon(\Delta(v))$ in $\Omega^{\prime}$. Let $\Omega^{\prime \prime}$ be obtained from $\Omega^{\prime}$ by a complete entire transformation. Then we have that $\varepsilon(\mu)=\varepsilon(\Delta(\mu))$ in $\Omega^{\prime \prime}$. Notice that since the generalised equation $\Omega$ repeats infinitely many times in the infinite branch, the tribe $t(\mu)$ of $\mu$ is minimal in $\Omega^{\prime \prime}$. Without loss of generality, let $\alpha(\mu)<\alpha(\Delta(\mu))$. From the above argument, it follows that the word $H^{\prime \prime}[\alpha(\mu), \alpha(\Delta(\mu))-1]$ is irreducible.

Therefore, since $G_{R(\Omega)} \simeq G_{R\left(\Omega^{\prime}\right)} \simeq G_{R\left(\Omega^{\prime \prime}\right)}$, the statement follows.

Recall that quadratic words of the type $[x, y], x^{2}, z^{-1} c z$, where $c$ is a constant are called atomic quadratic words or simply atoms. Let $W=1$ be a quadratic equation over $G$ written in the form $r_{1} r_{2} \cdots r_{k}=d$, where $r_{i}$ are atoms and $d \in G$. The 
number $k$ is called the rank of the quadratic equation. Suppose that the rank $k$ of $W=1$ is greater than or equal to 2 . A solution $H$ of $W$ is atom-commutative if $\left[H\left(r_{i}\right), H\left(r_{i+1}\right)\right]=1$ for all $i=1, \ldots, k-1$.

Suppose that the quadratic equation satisfies one of the following two alternatives:

- The Euler characteristic of $W$ is at most -2 , or $W$ corresponds to a punctured torus and the subgroup

$$
\left.\left\langle\varphi_{0}(\eta), \varphi_{0}\left(h_{i}^{(0)}\right)\right| \eta \in \mathcal{N}, h_{i}^{(0)} \text { is covered by a quadratic-coefficient base }\right\rangle
$$

of $\mathfrak{T}_{1}$ is nonabelian, ie the retraction of the (punctured) surface onto $\mathfrak{T}_{1}$ is nonabelian.

- The rank $k$ of $W$ is greater than or equal to 2 and the minimal solution $\varphi_{0}$ is not atom-commutative.

Note that, in the case of free groups, the above conditions are sufficient to ensure the radical of the quadratic equation $W=1$ coincides with the normal closure [38; 52]. We will see in the next section that the same result holds for arbitrary partially commutative groups (under the condition that the set of solutions of the quadratic equation factors through an infinite branch).

Notice that, by Lemma 6.2, we have that $\mathbb{K}^{\perp}$ is either $E_{d}(\Gamma)$-directly indecomposable or $E_{c}(\Gamma)$-free abelian. If the equation $W$ satisfies one of the above alternatives, then $\mathbb{K}^{\perp}$ cannot be free abelian and hence it is directly indecomposable.

Define the graph $\Gamma_{0}$ by

- $V\left(\Gamma_{0}\right)=V\left(\Gamma_{1}\right) \cup\left\{x_{1}^{(0)}, \ldots, x_{n}^{(0)}\right\}$,

- $E_{c}\left(\Gamma_{0}\right)=E_{c}\left(\Gamma_{1}\right)$,

- $E_{d}\left(\Gamma_{0}\right)=E_{d}\left(\Gamma_{1}\right) \cup\left\{\left(x_{i}^{(0)}, x_{j}^{(1)}\right) \mid\right.$ for all $\left.x_{j}^{(1)} \in \mathbb{K}, i=1, \ldots, n\right\}$,

where $n=|\mathcal{N}|$. Then the group $\mathbb{H}_{0}=\mathbb{H}\left(\Gamma_{0}\right)$ is given by the presentation

$$
\left.\left\langle\mathbb{H}_{1}, x_{1}^{(0)}, \ldots, x_{n}^{(0)}\right|\left[x_{i}^{(0)}, x_{j}^{(1)}\right]=1 \text {, for all } x_{j}^{(1)} \in \mathbb{K}, i=1, \ldots, n\right\rangle .
$$

Define the map $\tau_{0}^{\prime}$ as

$$
\begin{cases}\eta_{i} \rightarrow x_{i}^{(0)} & \eta_{i} \in \mathcal{N}, \\ \Delta\left(\eta_{i}\right) \rightarrow x_{i}^{(0)} & \eta_{i} \in \mathcal{N}, \eta_{i}=\Delta\left(\eta_{i}\right)^{\epsilon}, \epsilon \in\{ \pm 1\} \\ v \rightarrow \varphi_{0}(h(v)) & \text { for all quadratic-coefficient bases } v, \\ h_{i}^{(0)} \rightarrow \varphi_{0}\left(h_{i}^{(0)}\right) & \text { for all nonactive items } h_{i}^{(0)}\end{cases}
$$


If $\mathfrak{T}_{1}=\left\langle y^{(1)} \mid S_{1}\right\rangle$, then we set $\mathfrak{T}_{0}$ to be the quotient of $\mathbb{H}_{0}$ by the set of relations $S_{1}$ and the set of relations $S$ consisting of the set of basic relations $\left[C_{\mathfrak{T}_{1}}\left(\mathbb{K}^{\perp}\right), x_{i}^{(0)}\right]=1$, $1 \leq i \leq n$ and the relation $\tau_{0}^{\prime}(W)$, where $W$ is a quadratic word in the normal form from Corollary 4.7. Notice that $\tau_{0}^{\prime}(W)$ is a quadratic word in the normal form in variables $x_{i}^{(0)}, i=1, \ldots, n$.

Lemma 6.6 The map $\tau_{0}^{\prime}$ extends via the isomorphism $\psi$ (see Lemma 4.4) to a homomorphism $\tau_{0}$ from $G_{\Omega_{0}}$ to $\mathfrak{T}_{0}$.

Proof By Corollary 4.7, the group $G_{\Omega_{0}}$ is isomorphic to the group $\mathcal{K}$. We show that $\tau_{0}^{\prime}$ induces a homomorphism from $\mathcal{K}$ to $\mathfrak{T}_{0}$.

It is immediate to check that if $r$ is either the relation $W=1$ or a relation of the type $v^{\varepsilon(v)}=\Delta(v)^{\varepsilon(\Delta(v))}$ or a relation from $R^{\prime}$, then $\tau_{0}^{\prime}(r)=1$. Therefore, we are left to show that $\tau_{0}^{\prime}$ is trivial on the set of commutators $\Xi$.

Assume that $\left(\left[v_{i}, v_{j}\right]=1\right) \in \Xi$ where $v_{i}, v_{j}$ are short bases. Since bases from the quadratic part belong to tribes that dominate the minimal tribe, it follows that $v_{i}$ and $v_{j}$ belong to a tribe that strictly dominates the minimal tribe and hence, since we assume that $\Omega_{0}=\Omega_{0}^{\prime}$, the bases $v_{i}$ and $v_{j}$ are quadratic-coefficient bases. Since $\tau_{0}^{\prime}\left(\left[v_{i}, v_{j}\right]\right)=\varphi_{0}\left(\left[v_{i}, v_{j}\right]\right)$ and $\varphi_{0}$ is a homomorphism, we have that $\tau_{0}^{\prime}\left(\left[v_{i}, v_{j}\right]\right)=1$.

Similarly, if $\left(\left[v_{i}, h_{j}^{(0)}\right]=1\right) \in \Xi$, where $v_{i}$ is a quadratic-coefficient base and $h_{j}^{(0)}$ is a nonactive item, then $\tau_{0}^{\prime}\left(\left[v_{i}, h_{j}^{(0)}\right]\right)=\varphi_{0}\left(\left[v_{i}, h_{j}^{(0)}\right]\right)=1$.

If $\left(\left[h_{i}^{(0)}, h_{j}^{(0)}\right]=1\right) \in \Xi$, where $h_{i}^{(0)}$ and $h_{j}^{(0)}$ belong to the nonactive part, then we have that $\tau_{0}^{\prime}\left(\left[h_{i}^{(0)}, h_{j}^{(0)}\right]\right)=\varphi_{0}\left(\left[h_{i}^{(0)}, h_{j}^{(0)}\right]\right)=1$.

Assume that $\left(\left[v_{i}, h_{j}^{(0)}\right]=1\right) \in \Xi$, where $v_{i}$ is a quadratic base and $h_{j}^{(0)}$ is an item from the nonactive part. By the definition of $\Xi$ and the fact that $\Re_{\Upsilon_{0}}$ is completed, one has that $\Re \Upsilon_{0}\left(h_{i}^{(0)}, h_{j}^{(0)}\right)$ for all $h_{i}^{(0)}$ from the quadratic part of $\Omega_{0}$. Hence, from the description of the process, we conclude that $\Re_{\Upsilon_{1}}\left(h_{i}^{(1)}, h_{j}^{(1)}\right)$ for all $h_{i}^{(1)}$ from the word $\pi\left(v_{0}, v_{1}\right)\left(h_{i}^{(0)}\right)$ and for all $h_{j}^{(1)}$ from the word $\pi\left(v_{0}, v_{1}\right)\left(h_{j}^{(0)}\right)$. By definition of a solution of a generalised equation, it follows that for any solution $H^{(1)}$ of $\Omega_{1}$, $H_{i}^{(1)} \leftrightarrows H_{j}^{(1)}$. By the induction hypothesis (IH2) on $\tau_{1}$, for all $y_{i}$ from the word $\tau_{1}\left(h_{i}^{(1)}\right)$ and all $y_{j}$ from the word $\tau_{1}\left(h_{j}^{(1)}\right)$, we have that $H^{\prime}\left(y_{i}\right) \leftrightarrows H^{\prime}\left(y_{j}\right)$. By the induction hypothesis (IH1) on $\mathbb{H}_{1}$, it follows that $\left(x_{i}, x_{j}\right) \in E_{d}\left(\Gamma_{1}\right)$. Therefore, for any $x_{j}$ from the word $\varphi_{0}\left(h_{j}^{(0)}\right)$ and any $x_{i}$ from the word $\varphi_{0}\left(h_{i}^{(0)}\right)$, where $h_{i}^{(0)}$ belongs to the quadratic part, we have that $\left(x_{i}, x_{j}\right) \in E_{d}\left(\Gamma_{1}\right)$ and so $x_{j} \in \mathbb{A}\left(h^{(0)}(\eta)\right)$ for every base $\eta$ from the quadratic part. Thus, $\varphi_{0}\left(h_{j}^{(0)}\right) \in \mathbb{K}$. We conclude that $\tau_{0}\left(\left[v_{i}, h_{j}^{(0)}\right]\right)=1$. This shows that $\tau_{0}$ is a homomorphism. 
Lemma 6.7 The homomorphism $\tau_{0}: G_{\Omega_{0}} \rightarrow \mathfrak{T}_{0}$ makes diagram (11) commutative.

Proof Notice that any solution that factors through the branch is the composition of an automorphism associated to the vertex $v_{0}$, the epimorphism $\pi\left(v_{0}, v_{1}\right)$ and a solution of $\Omega_{1}$. Since by [14, Lemma 7.12], all the automorphisms fix the subgroup $N Q$ of $G_{\Omega_{0}}$ generated by the items covered by quadratic-coefficient bases and the nonactive items, it follows that the restriction of any solution $H^{(0)}$ that factors through the branch to this subgroup coincides with a solution $H^{(1)}$ on the subgroup $\pi\left(v_{0}, v_{1}\right)(N Q)$ of $G_{\Omega_{1}}$, ie for all items $h_{i}^{(0)} \in N Q$ we have that $H_{i}^{(0)}=H^{(0)}\left(h_{i}^{(0)}\right)=H^{(1)}\left(\pi\left(v_{0}, v_{1}\right)\left(h_{i}^{(0)}\right)\right)$. By induction hypothesis, there exists a homomorphism $H^{(1)^{\prime}}$ from $\mathfrak{T}_{1}$ that makes diagram (11) commutative. We define $H^{(0)^{\prime}}$ on the subgroup $\mathfrak{T}_{1}$ of $\mathfrak{T}_{0}$ to be $H^{(1)^{\prime}}$ and set $H^{(0)^{\prime}}\left(x_{i}^{(0)}\right)=H^{(0)}\left(\eta_{i}\right)$, where $\eta_{i} \in \mathcal{N}$.

It suffices to show that $H^{(0)^{\prime}}$ is a homomorphism. In this case, the commutativity of the diagram follows by construction. Let us show that $H^{(0)^{\prime}}\left(\left[x_{i}^{(0)}, C_{\mathfrak{T}_{1}}\left(\mathbb{K}^{\perp}\right)\right]\right)=1$. From the definition of $\mathbb{K}$ and the induction hypothesis on the tower $\left(\mathfrak{T}_{1}, \mathbb{H}_{1}\right)$, we have that

$$
H^{(1)^{\prime}}\left(\varphi_{0}(\eta)\right)=H^{(1)}\left(\pi\left(v_{0}, v_{1}\right)(\eta)\right) \leftrightarrows H^{(1)^{\prime}}(\mathbb{K})
$$

Since every solution $H^{(0)}$ that factors through the branch is obtained from a solution $H^{(1)}$ that factors through the branch by precomposing it with a canonical automorphism (and $\pi\left(v_{0}, v_{1}\right)$ ), we conclude that $a_{i} \leftrightarrows\left\{H^{(0)}(\eta), \eta \in \mathcal{N}\right\}$ for all solutions $H^{(0)}$ that factor through the branch if and only if $a_{i} \leftrightarrows\left\{H^{(1)}\left(\pi\left(v_{0}, v_{1}\right)(h(\eta))\right), \eta \in \mathcal{N}\right\}$ for all $H^{(1)}$ that factor through the branch, where $a_{i} \in \mathbb{G}$. It follows that

$$
H^{(0)}\left(\eta_{i}\right)=H^{(0)^{\prime}}\left(x_{i}^{(0)}\right) \leftrightarrows H^{(1)^{\prime}}(\mathbb{K})=H^{(0)^{\prime}}(\mathbb{K})
$$

and so

$$
H^{(0)^{\prime}}\left(x_{i}^{(0)}\right) \in\left\langle\operatorname{alph}\left(H^{(0)^{\prime}}(\mathbb{K})\right)^{\perp}\right\rangle .
$$

By the induction hypothesis, $\left\langle\operatorname{alph}\left(H^{(1)^{\prime}}(\mathbb{K})\right)^{\perp}\right\rangle=\left\langle\operatorname{alph}\left(H^{(1)^{\prime}}\left(\mathbb{K}^{\perp}\right)\right)\right\rangle$ and hence we have that $\left[H^{(0)^{\prime}}\left(x_{i}^{(0)}\right), C_{\mathbb{G}}\left(H^{(0)^{\prime}}\left(\mathbb{K}^{\perp}\right)\right)\right]=1$.

Finally, direct computation shows that $H^{(0)^{\prime}}(W)=H^{(0)}(W)=1$. This proves $H^{(0)^{\prime}}$ is a homomorphism.

Lemma 6.8 The group $\mathbb{H}_{0}$ satisfies (IH1): for every $x_{i}, x_{j} \in \mathbb{H}_{0}$, we have that $\left(x_{i}, x_{j}\right) \in E_{d}\left(\Gamma_{0}\right)$ if and only if $H^{(0)^{\prime}}\left(x_{i}\right) \leftrightarrows H^{(0)^{\prime}}\left(x_{j}\right)$ for all homomorphisms $H^{(0)^{\prime}}$ induced by a solution $H^{(0)}$ that factors through the branch. Furthermore, we have that if $\left(x_{i}, x_{j}\right) \in E_{c}\left(\Gamma_{0}\right)$, then $H^{(0)^{\prime}}\left(x_{i}\right), H^{(0)^{\prime}}\left(x_{j}\right)$ belong to a cyclic subgroup for all homomorphisms $H^{(0)^{\prime}}$ induced by a solution $H^{(0)}$ that factors through the branch. 
Proof If $x_{i}, x_{j} \in \mathbb{H}_{1}<\mathbb{H}_{0}$, then the statement follows by the induction hypothesis (IH1) for $\mathbb{H}_{1}$.

By definition $\left(x_{i}^{(0)}, x_{j}^{(0)}\right) \notin E_{d}\left(\Gamma_{0}\right)$, for all $1 \leq i<j \leq n$. Let us show that $H^{(0)^{\prime}}\left(x_{i}^{(0)}\right) \not H^{(0)^{\prime}}\left(x_{j}^{(0)}\right)$. Notice that by definition, $H^{(0)^{\prime}}\left(x_{i}^{(0)}\right)=H\left(\eta_{i}\right)$, where $\eta_{i} \in \mathcal{N}$. Since all the bases from $\mathcal{N}$ belong to the same tribe, it follows that $H^{(0)}\left(\eta_{i}\right) \not \rightarrow$ $H^{(0)}\left(\eta_{j}\right)$.

By definition $\left(x_{i}^{(0)}, x_{j}^{(1)}\right) \in E_{d}\left(\Gamma_{0}\right)$, for all $x_{j}^{(1)} \in \mathbb{K}, i=1, \ldots, n$. From the definition of $\mathbb{K}$ and the induction hypothesis (IH1) for the graph tower $\left(\mathfrak{T}_{1}, \mathbb{H}_{1}\right)$, we have that $H^{(1)^{\prime}}\left(\varphi_{0}(\eta)\right)=H^{(1)}\left(\pi\left(v_{0}, v_{1}\right)(\eta)\right) \leftrightarrows H^{(1)^{\prime}}(\mathbb{K})$. As we have already seen in Lemma 6.7, we have that $a_{i} \leftrightarrows\left\{H^{(0)}(\eta), \eta \in \mathcal{N}\right\}$ for all solutions $H^{(0)}$ that factor through the branch if and only if $a_{i} \leftrightarrows\left\{H^{(1)}\left(\pi\left(v_{0}, v_{1}\right)(h(\eta))\right), \eta \in \mathcal{N}\right\}$ for all solutions $H^{(1)}$ that factor through the branch, where $a_{i} \in \mathbb{G}$.

Assume that for $x_{j}^{(1)} \in \mathbb{H}_{1}$ we have $H^{(0)^{\prime}}\left(x_{j}^{(1)}\right) \leftrightarrows H^{(0)^{\prime}}\left(x_{i}^{(0)}\right)$ for some $i=1, \ldots, n$. Since by definition $H^{(0) \prime}\left(x_{i}^{(0)}\right)=H^{(0)}\left(\eta_{i}\right)$ and all the quadratic bases belong to the same minimal tribe, it follows that $H^{(0)^{\prime}}\left(x_{j}^{(1)}\right) \leftrightarrows H^{(0)^{\prime}}\left(x_{i}^{(0)}\right)$ for all $i=1, \ldots, n$. Since the sets $\left\{\pi\left(v_{0}, v_{1}\right)(h(\eta)), \eta \in \mathcal{N}\right\}$ and $\{h(\eta), \eta \in \mathcal{N}\}$ define the same minimal tribe, we conclude that

$$
\begin{aligned}
H^{(0)^{\prime}}\left(x_{j}^{(1)}\right)=H^{(1)^{\prime}}\left(x_{j}^{(1)}\right) & \\
& \leftrightarrows H^{(0)}\left(\pi\left(v_{0}, v_{1}\right)\left(h\left(\eta_{i}\right)\right)\right)=H^{(0)^{\prime}}\left(\varphi_{0}\left(h\left(\eta_{i}\right)\right)\right)=H^{(1)^{\prime}}\left(\varphi\left(h\left(\eta_{i}\right)\right)\right)
\end{aligned}
$$

for all $i=1, \ldots, n$. By the induction hypothesis (IH1) on $\mathbb{H}_{1}$, it follows that $x_{j}^{(1)} \in$ $\mathbb{A}\left(\varphi\left(h\left(\eta_{i}\right)\right)\right)=\mathbb{K}$. Therefore, $\left(x_{j}^{(1)}, x_{i}^{(0)}\right) \in E_{d}\left(\Gamma_{0}\right)$.

Finally, since all the edges $\left(x_{i}, x_{j}\right)$ from $E_{c}\left(\Gamma_{0}\right)$ belong to $E_{c}\left(\Gamma_{1}\right)$, by induction on $\mathbb{H}_{1}$ and definition of $H^{(0)^{\prime}}$, we conclude that for every solution $H^{(0)}$ that factors through the branch, $H^{(0)^{\prime}}\left(x_{i}\right)=H^{(1)^{\prime}}\left(x_{i}\right)$ and $H^{(0)^{\prime}}\left(x_{j}\right)=H^{(1)^{\prime}}\left(x_{j}\right)$ belong to the same cyclic subgroup.

Lemma 6.9 The homomorphism $\tau_{0}$ satisfies the induction hypothesis (IH2): for all $h_{i}^{(0)}$, if $\tau_{0}\left(h_{i}^{(0)}\right)=y_{i 1} \cdots y_{i k}$, then we have that $\mathbb{A}\left(H^{(0)^{\prime}}\left(y_{i j}\right)\right) \supset \mathbb{A}\left(H_{i}^{(0)}\right)$ and $\bigcap_{j=1}^{k} \mathbb{A}\left(H^{(0) \prime}\left(y_{i j}\right)\right)=\mathbb{A}\left(H_{i}^{(0)}\right)$ for solutions that factor through the branch.

Proof If the item $h_{i}^{(0)}$ is covered by a quadratic-coefficient base or it is nonactive, then $\tau_{0}\left(h_{i}^{(0)}\right)=\tau_{1} \pi\left(v_{0}, v_{1}\right)\left(h_{i}^{(0)}\right)$. By construction, the epimorphism $\pi\left(v_{0}, v_{1}\right)$ satisfies the statement of the lemma, and by induction hypothesis so does $\tau_{1}$. It follows that the statement of the lemma holds for $\tau_{0}\left(h_{i}^{(0)}\right)$. 
We are left to consider the case when $h_{i}^{(0)}$ belongs to a quadratic base and is not covered by a quadratic-coefficient base. In this case, $h_{i}^{(0)}$ is covered by two long bases and, hence belongs to a minimal tribe. Then, by definition, $\tau_{0}\left(h_{i}^{(0)}\right)=\tau_{0}^{\prime}\left(\psi\left(h_{i}^{(0)}\right)\right)$. If $\psi\left(h_{i}^{(0)}\right)=w_{i}(\mathcal{N}, C)$ where $C$ is the set of items covered by quadratic-coefficient bases, then $\tau_{0}\left(h_{i}^{(0)}\right)=w_{i}\left(x_{i}^{(0)}, \varphi_{0}(C)\right)=y_{i 1} \cdots y_{i k}$. Since we have that $H^{(0)^{\prime}}\left(\tau_{0}\left(h_{i}^{(0)}\right)\right)=$ $w_{i}\left(H^{(0)}\left(\eta_{i}\right), H^{(0)}(C)\right)$ and all the bases and items of the quadratic part belong to tribes that dominate the minimal tribe, it follows that $\mathbb{A}\left(H^{(0)^{\prime}}\left(y_{i j}\right)\right) \supset \mathbb{A}\left(H_{i}^{(0)}\right)$. Furthermore, since the item $h_{i}^{(0)}$ belongs to a quadratic base, it follows that $y_{i l}=x_{i}^{(0)}$ for some $l=1, \ldots, k$ and some $i=1, \ldots, n$. Since $x_{i}^{(0)}$ belongs to the minimal tribe, we have that $\bigcap_{j=1}^{k} \mathbb{A}\left(H^{(0)^{\prime}}\left(y_{i j}\right)\right)=\mathbb{A}\left(H_{i}^{(0)}\right)$.

Let us now deal with the exceptional cases.

Rank one Assume that $W$ corresponds to a torus, ie $W=\left[\eta_{1}, \eta_{2}\right]=1$. Define the graph $\Gamma_{0}$ as follows. If $\mathbb{K}^{\perp}$ is nonabelian, set

- $V\left(\Gamma_{0}\right)=V\left(\Gamma_{1}\right) \cup\left\{x_{1}^{(0)}, x_{2}^{(0)}\right\}$;

- $E_{c}\left(\Gamma_{0}\right)=E_{c}\left(\Gamma_{1}\right) \cup\left\{\left(x_{1}^{(0)}, x_{2}^{(0)}\right)\right\}$;

- $E_{d}\left(\Gamma_{0}\right)=E_{d}\left(\Gamma_{1}\right) \cup\left\{\left(x_{i}^{(0)}, x_{j}^{(1)}\right) \mid\right.$ for all $\left.x_{j}^{(1)} \in \mathbb{K}, i=1,2\right\}$.

If $\mathbb{K}^{\perp}$ is abelian, then set

- $V\left(\Gamma_{0}\right)=V\left(\Gamma_{1}\right) \cup\left\{x_{1}^{(0)}, x_{2}^{(0)}\right\}$;

- $E_{c}\left(\Gamma_{0}\right)=E_{c}\left(\Gamma_{1}\right) \cup\left\{\left(x_{1}^{(0)}, x_{2}^{(0)}\right)\right\} \cup\left\{\left(x_{i}^{(0)}, x_{j}^{(1)}\right) \mid\right.$ for all $\left.x_{j}^{(1)} \in \mathbb{K}^{\perp}, i=1,2\right\}$;

- $E_{d}\left(\Gamma_{0}\right)=E_{d}\left(\Gamma_{1}\right) \cup\left\{\left(x_{i}^{(0)}, x_{j}^{(1)}\right) \mid\right.$ for all $\left.x_{j}^{(1)} \in \mathbb{K}, i=1,2\right\}$.

If $\mathfrak{T}_{1}=\left\langle y^{(1)} \mid S_{1}\right\rangle$, then we set $\mathfrak{T}_{0}$ to be the quotient of $\mathbb{H}_{0}$ by the set of relations $S_{1}$ and the set of basic relations $S$ :

$$
\left[C_{\mathfrak{T}_{1}}\left(\mathbb{K}^{\perp}\right), x_{i}^{(0)}\right]=1, \quad i=1,2 .
$$

Lemma 6.10 The map $\tau_{0}^{\prime}: \eta_{i} \mapsto x_{i}^{(0)}, i=1,2$, extends via the isomorphism $\psi$ (see Lemma 4.4) to a homomorphism $\tau_{0}$ from $G_{\Omega_{0}}$ to $\mathfrak{T}_{0}$ that makes diagram (11) commutative. Furthermore, $\mathfrak{T}_{0}, \mathbb{H}_{0}$ and $\tau_{0}$ satisfy the induction hypotheses (IH), (IH1) and $(\mathrm{IH} 2)$.

Proof The proof is analogous to the proofs of Lemmas 6.6, 6.7, 6.8, 6.9.

Notice that other quadratic equations of rank one do not define an infinite family of homomorphisms. Indeed, by Remark 6.1, any solution of a generalised equation is induced by a solution of the corresponding generalised equation over the free group. Now the claim follows from the description of the radical ideal for quadratic equations in the free group; see [37]. 
Other cases Finally, let us consider the remaining cases. Notice that if the image of the surface under the retraction is commutative, then the minimal solution is atomcommutative. Hence, without loss of generality, we assume that the minimal solution is atom-commutative.

Define the graph $\Gamma_{0}$ as follows. If $\mathbb{K}^{\perp}$ is nonabelian, set

- $V\left(\Gamma_{0}\right)=V\left(\Gamma_{1}\right) \cup\left\{x_{1}^{(0)}, \ldots, x_{n}^{(0)}\right\}$;

- $E_{c}\left(\Gamma_{0}\right)=E_{c}\left(\Gamma_{1}\right) \cup\left\{\left(x_{i}^{(0)}, x_{j}^{(0)}\right) \mid 1 \leq i<j \leq n\right\}$;

- $E_{d}\left(\Gamma_{0}\right)=E_{d}\left(\Gamma_{1}\right) \cup\left\{\left(x_{i}^{(0)}, x_{j}^{(1)}\right) \mid\right.$ for all $\left.x_{j}^{(1)} \in \mathbb{K}, i=1, \ldots, n\right\}$.

If $\mathbb{K}^{\perp}$ is abelian, then set

- $V\left(\Gamma_{0}\right)=V\left(\Gamma_{1}\right) \cup\left\{x_{1}^{(0)}, \ldots, x_{n}^{(0)}\right\} ;$

- $E_{c}\left(\Gamma_{0}\right)=E_{c}\left(\Gamma_{1}\right) \cup\left\{\left(x_{i}^{(0)}, x_{j}^{(0)}\right) \mid 1 \leq i<j \leq n\right\} \cup\left\{\left(x_{i}^{(0)}, x_{j}^{(1)}\right) \mid x_{j}^{(1)} \in \mathbb{K}^{\perp}, i=\right.$ $1, \ldots, n\}$

- $E_{d}\left(\Gamma_{0}\right)=E_{d}\left(\Gamma_{1}\right) \cup\left\{\left(x_{i}^{(0)}, x_{j}^{(1)}\right) \mid\right.$ for all $\left.x_{j}^{(1)} \in \mathbb{K}, i=1, \ldots, n\right\}$.

If $\mathfrak{T}_{1}=\left\langle y^{(1)} \mid S_{1}\right\rangle$, then we set $\mathfrak{T}_{0}$ to be the quotient of $\mathbb{H}_{0}$ by the set of relations $S_{1}$ and the set of relations $S$ defined as follows:

- If one of the elements $\varphi_{0}\left(\eta_{1} \cdots \eta_{2 g}\right), \varphi_{0}\left(\lambda_{i}\right), i=2 g+1, \ldots, m$ is nontrivial, then the set of relations $S$ is defined as

$$
S=\left\{x_{1}^{(0)} \cdots x_{2 g}^{(0)}=\varphi_{0}\left(\eta_{1} \cdots \eta_{2 g}\right),\left[x_{i}^{(0)}, C\right]=1, i=1, \ldots, n\right\},
$$

where

$$
C=C_{\mathfrak{T}_{1}}\left(\varphi_{0}\left(\lambda_{2 g+1}\right)^{\varphi_{0}\left(\eta_{2 g+1}\right)}, \ldots, \varphi_{0}\left(\lambda_{m}\right)^{\varphi_{0}\left(\eta_{m}\right)}, \varphi_{0}\left(\eta_{1} \cdots \eta_{2 g}\right)\right) .
$$

- Otherwise, $S=\left\{x_{1}^{(0)} \cdots x_{2 g}^{(0)}=1\right\} \cup\{$ the set of basic relations $\}$.

In these cases, define the map $\tau_{0}^{\prime}$ as follows:

$$
\begin{cases}\eta_{j} \rightarrow x_{j}^{(0)} & \eta_{j} \in \mathcal{N}, j=1, \ldots, 2 g, \\ \Delta\left(\eta_{j}\right) \rightarrow x_{j}^{(0)} & \eta_{j} \in \mathcal{N}, \eta_{j}=\Delta\left(\eta_{j}\right)^{\epsilon}, \epsilon= \pm 1, j=1, \ldots, 2 g, \\ \eta_{j} \rightarrow x_{j}^{(0)} \varphi_{0}\left(\eta_{j}\right) & \eta_{j} \in \mathcal{N}, j=2 g+1, \ldots, n, \\ \Delta\left(\eta_{j}\right) \rightarrow x_{j}^{(0)} \varphi_{0}\left(\eta_{j}\right) & \eta_{j} \in \mathcal{N}, \eta_{j}=\Delta\left(\eta_{j}\right)^{\epsilon}, \epsilon= \pm 1, j=2 g+1, \ldots, n, \\ \nu \rightarrow \varphi_{0}(h(v)) & \text { if } v \text { is a quadratic-coefficient base, } \\ h_{i}^{(0)} \rightarrow \varphi_{0}\left(h_{i}^{(0)}\right) & \text { if } h_{i}^{(0)} \text { is a nonactive item. }\end{cases}
$$


Lemma 6.11 The map $\tau_{0}^{\prime}$ extends via the isomorphism $\psi$ ( see Lemma 4.4) to a homomorphism $\tau_{0}$ from $G_{\Omega_{0}}$ to $\mathfrak{T}_{0}$ that makes diagram (11) commutative. Furthermore, $\mathfrak{T}_{0}, \mathbb{H}_{0}$ and $\tau_{0}$ satisfy the induction hypotheses (IH), (IH1) and (IH2).

Proof By Corollary 4.7, the group $G_{\Omega_{0}}$ is isomorphic to the group $\mathcal{K}$. We show $\tau_{0}^{\prime}$ induces a homomorphism from $\mathcal{K}$ to $\mathfrak{T}_{0}$.

It is immediate to check that $\tau_{0}^{\prime}(r)=1$ if $r$ is either the relation $W$, a relation of the type $v^{\varepsilon(v)}=\Delta(v)^{\varepsilon(\Delta(v))}$ or a relation from $R^{\prime}$.

Therefore, we are left to show that $\tau_{0}^{\prime}$ is trivial on the set of commutators $\Xi$; see (6). The proof is analogous to the proof of Lemma 6.6.

Let us now show that diagram (11) is commutative. Any solution that factors through the branch is the composition of an automorphism associated to the vertex, the epimorphism $\pi\left(v_{0}, v_{1}\right)$ and a solution of $\Omega_{1}$ that factors through the branch. Since by [14, Lemma 7.12] all the automorphisms fix the subgroup $N Q$ of $G_{R\left(\Omega_{0}\right)}$ generated by the items covered by quadratic-coefficient bases and the nonactive items, it follows that the restriction of any solution $H^{(0)}$ that factors through the branch onto this subgroup coincides with a solution $H^{(1)}$ on the subgroup $\pi\left(v_{0}, v_{1}\right)(N Q)$ of $G_{\Omega_{1}}$, ie for all items $h_{i}^{(0)} \in N Q$ we have that $H^{(0)}\left(h_{i}^{(0)}\right)=H^{(1)}\left(\pi\left(v_{0}, v_{1}\right)\left(h_{i}^{(0)}\right)\right)$. By induction hypothesis, there exists a homomorphism $H^{(1)^{\prime}}$ from $\mathfrak{T}_{1}$ that makes diagram (11) commutative. We define $H^{(0)^{\prime}}$ on the subgroup $\mathfrak{T}_{1}$ of $\mathfrak{T}_{0}$ to be $H^{(1)^{\prime}}$ and we define $H^{(0)^{\prime}}\left(x_{j}^{(0)}\right)=H^{(0)}\left(\eta_{j}\right)$, if $\eta_{j} \in \mathcal{N}$ and $j=1, \ldots, 2 g$, and $H^{(0)^{\prime}}\left(x_{j}^{(0)}\right)=$ $H^{(0)}\left(\eta_{j}\right) H^{(0)}\left(\varphi_{0}\left(\eta_{j}\right)\right)^{-1}$, if $\eta_{j} \in \mathcal{N}$ and $j=2 g+1, \ldots, n$.

It suffices to show that $H^{(0)^{\prime}}$ is a homomorphism. In this case, the commutation of diagram (11) follows by construction. Since, by assumption, the minimal solution $\varphi_{0}\left(\eta_{i}\right)$ is atom-commutative and since any solution that factors through the branch is obtained from the minimal solution by precomposing it with an automorphism, it follows that any solution that factors through the branch is also atom-commutative. Furthermore, notice that by Remark 6.4, the image of any item under the homomorphism $H^{(0)}$ is a block element such that $\mathbb{A}\left(H^{(0)}\left(\eta_{i}\right)\right)=\mathbb{A}\left(\mathbb{G}_{\mathbb{K}^{\perp}}\right)$, for all $i=1, \ldots, n$. By Lemma 6.5, there exists an element $U$ so that $H^{(0)}(U)$ is irreducible. Hence, the images of all the atoms under $H^{(0)}$ belong to the same cyclic subgroup:

$$
\left\langle\sqrt{H^{(0)}\left(\lambda_{2 g+1}^{\eta_{2 g+1}}\right)}\right\rangle=\cdots=\left\langle\sqrt{H^{(0)}\left(\lambda_{n}^{\eta_{n}}\right)}\right\rangle=\left\langle\sqrt{H^{(0)}\left(\eta_{1} \cdots \eta_{2 g}\right)}\right\rangle .
$$

We conclude that $H^{(0)}\left(\left[x_{i}^{(0)}, C\right]\right)=1$. Therefore, diagram (11) is commutative.

The proofs that the induction hypotheses (IH1) and (IH2) hold, are analogous to the proofs of Lemmas 6.8 and 6.9, correspondingly. 
Notice that since $H^{(0)}\left(x_{1}\right)$ is cyclically reduced, the argument given above shows that if the quadratic equation is nonorientable (ie it contains an atom $x_{1}^{2}$ ), then the word $H^{(0)}\left(c^{x_{i}}\right)$ is cyclically reduced. Hence, we conclude that in this case the retraction of the surface is abelian.

We summarise the results of this section in the proposition below.

Proposition 6.12 Let $\Omega_{0} \rightarrow \Omega_{1} \rightarrow \cdots \rightarrow \Omega_{q}$ be a branch of a tribal solution tree and assume that $\Omega_{1}$ satisfies the induction hypotheses (IH), (IH1) and (IH2); see page 784. If $\Omega_{0}=\langle\Upsilon, \Re \Upsilon\rangle$ is a tribal generalised equation of type 12, then there exists a graph tower $\left(\mathfrak{T}_{0}, \mathbb{H}_{0}\right)$ and a homomorphism $\tau_{0}$ from $G_{\Omega_{0}}$ to $\mathfrak{T}_{0}$ such that for every solution that factors through the branch, there exists a homomorphism from $\mathfrak{T}_{0}$ to $\mathbb{G}$ that makes diagram (11) commutative.

\subsection{Singular and strongly singular periodic structures}

In this and the following sections we use the notation and results from [14, Chapter 6], of which we now give a very brief and informal summary.

Informally, a periodic structure $\mathcal{P}$ is a set of items, bases and sections of a generalised equation $\Omega$ that imposes restrictions on the set of solutions of $\Omega$, namely that solutions are $P$-periodic and the image of items, bases and section from the periodic structure are "long" (they are subwords of $P^{n}$ of length greater than $2|P|$ ).

Given a periodic structure on $\Omega$, we study the corresponding coordinate group by introducing a new set of generators and exhibiting the corresponding presentation. In order to define the new set of generators, one constructs a graph $\Gamma=\Gamma(\mathcal{P})$, whose edges are labelled by items that belong to a section of the periodic structure. Without loss of generality, one can assume that $\Gamma$ is connected. One then chooses a maximal subforest of $\Gamma$ so that the edges are labelled by items that belong to a section from $\mathcal{P}$, but the items themselves do not belong to $\mathcal{P}$. We then complete the forest to a maximal subtree $T$ of $\Gamma$. The free group generated by the items that label edges of $\Gamma$ is generated by the items that label edges of $T$ and cycles $\mathfrak{c}_{e}$, where $e \in \Gamma \backslash T$.

Under the assumption that $\Omega$ is periodised with respect to $\mathcal{P}$ (ie words defined by cycles of $\Gamma$ based at a given point commute), one can choose a basis $C^{(1)} \cup C^{(2)}$ of the subgroup generated by the cycles $\mathfrak{c}_{e}$. For each edge $e_{i} \in T$ labelled by an item that belongs to $\mathcal{P}$, we consider two families of cycles $u_{i, e}, z_{i, e}$ defined by edges $e \notin T$ labelled by items that do not belong to $\mathcal{P}$. The cycles $u_{i, e}, z_{i, e}$ are based at the origin and the terminus of $e_{i}$, correspondingly. We prove that the set 
- $\bar{t}$ of items that do not belong to sections from $\mathcal{P}$,

- $\{h(e), e \in T, h(e) \notin \mathcal{P}\}$,

- $\left\{h\left(e_{1}\right), \ldots, h\left(e_{m}\right), e_{i} \in T, h\left(e_{i}\right) \in \mathcal{P}\right\}$,

- $\left\{u_{i e}, z_{i e}, i=1, \ldots, m, e \notin T, h(e) \notin \mathcal{P}\right\}$,

- $C^{(1)}, C^{(2)}$,

is a generating set of the group $G_{\Omega}$ and in this generating set, the system of equations $\Upsilon$ is equivalent to the union of the two systems of equations

$$
\begin{cases}u_{i e}^{h\left(e_{i}\right)}=z_{i e} & \text { where } e \in T, e \in \mathrm{Sh}, 1 \leq i \leq m, \\ {\left[u_{i e_{1}}, u_{i e_{2}}\right]=1} & \text { where } e_{j} \in T, e_{j} \in \mathrm{Sh}, j=1,2,1 \leq i \leq m, \\ {\left[h\left(\mathfrak{c}_{1}\right), h\left(\mathfrak{c}_{2}\right)\right]=1} & \text { where } \mathfrak{c}_{1}, \mathfrak{c}_{2} \in C^{(1)} \cup C^{(2)},\end{cases}
$$

and a system

$$
\Psi\left(\{h(e) \mid e \in T, e \in \operatorname{Sh}\}, h\left(C^{(1)}\right), \bar{t}, \bar{u}, \bar{z}, \mathcal{A}\right)=1,
$$

such that neither $h\left(e_{i}\right), 1 \leq i \leq m$, nor $h\left(C^{(2)}\right)$ occurs in $\Psi$; see [14, page 81] for details and notation.

Recall that a generalised equation $\Omega$ is called periodised (with respect to a given periodic structure) if for every two cycles $\mathfrak{c}_{1}$ and $\mathfrak{c}_{2}$ based at the same vertex in the graph $\Gamma$ commute in the coordinate group; see [14, Definition 6.9].

Definition 6.13 Let $\Omega$ be a generalised equation and let $\langle\mathcal{P}, R\rangle$ be a connected periodic structure on $\Omega$. We say that the generalised equation $\Omega$ is strongly singular with respect to the periodic structure $\langle\mathcal{P}, R\rangle$ if one of the following conditions holds.

(a) The generalised equation $\Omega$ is not periodised with respect to the periodic structure $\langle\mathcal{P}, R\rangle$.

(b) The generalised equation $\Omega$ is periodised with respect to the periodic structure $\langle\mathcal{P}, R\rangle$ and there exists an automorphism $\varphi$ of the coordinate group $G_{R(\Upsilon)}$ of the form described in parts [14, Lemma 6.14(2) and (3)], such that $\varphi$ does not induce an automorphism of $G_{R(\Omega)}$.

We say that the generalised equation $\Omega$ is singular with respect to the periodic structure $\langle\mathcal{P}, R\rangle$ if $\Omega$ is not strongly singular with respect to the periodic structure $\langle\mathcal{P}, R\rangle$ and one of the following conditions holds.

(a) The set $C^{(2)}$ has more than one element.

(b) The set $C^{(2)}$ has exactly one element, and (in the above notation) there exists a cycle $\mathfrak{c}_{e_{0}} \in\left\langle C^{(1)}\right\rangle, h\left(e_{0}\right) \notin \mathcal{P}$ such that $h\left(\mathfrak{c}_{e_{0}}\right) \neq 1$ in $G_{R(\Omega)}$. 
Otherwise, we say that $\Omega$ is regular with respect to the periodic structure $\langle\mathcal{P}, R\rangle$. In particular, if $\Omega$ is singular or regular with respect to the periodic structure $\langle\mathcal{P}, R\rangle$, then $\Omega$ is periodised.

When no confusion arises, instead of saying that $\Omega$ is (strongly) singular (or regular) with respect to the periodic structure $\langle\mathcal{P}, R\rangle$, we say that the periodic structure $\langle\mathcal{P}, R\rangle$ is (strongly) singular (or regular).

Suppose that $v_{0}$ is of type 2 , ie solutions that factor through the branch are $P$-periodic and $\Omega_{v_{0}}$ is either singular or strongly singular with respect to the corresponding periodic structure $\langle\mathcal{P}, R\rangle$.

To simplify the notation, set $\Omega_{i}=\Omega_{v_{i}}=\left\langle\Upsilon_{i}, \Re_{\Upsilon_{i}}\right\rangle$. Recall that $G_{\Omega_{i}}=\left\langle h^{(i)}\right\rangle, i=1,2$, $\mathbb{H}_{1}=\left\langle x^{(1)}\right\rangle$, and $\mathfrak{T}_{1}=\left\langle y^{(1)}\right\rangle$.

If the periodic structure $\langle\mathcal{P}, R\rangle$ is strongly singular, then we set $\mathfrak{T}_{0}=\mathfrak{T}_{1}$ and $\tau_{0}$ to be $\pi\left(v_{0}, v_{1}\right) \tau_{1}$, where $\tau_{1}$ is the homomorphism from $G_{\Omega_{1}}$ to $\mathfrak{T}_{1}$ that makes diagram (11) commutative. By [14, Lemma 6.17], any homomorphism that factors through the branch also factors through $G_{R\left(\Omega_{1}\right)}$ and so $\tau_{0}, \mathfrak{T}_{0}$ and $\mathbb{H}_{0}$ make diagram (11) commutative and satisfy the induction hypothesis.

Let us now assume that the periodic structure $\langle\mathcal{P}, R\rangle$ is singular.

The set of elements

$$
\left\{h^{(0)}(e) \mid e \in T\right\} \cup\left\{h^{(0)}\left(\mathfrak{c}_{e}\right) \mid e \notin T\right\}
$$

forms a basis of the free group $F\left(h^{(0)}\right)$ generated by

$$
\left\{h_{k}^{(0)} \mid h_{k}^{(0)} \in \sigma, \sigma \in \mathcal{P}\right\}
$$

Suppose first that the periodic structure is singular of type (a), ie the set $C^{(2)}$ has more than one element. Denote by $\varphi_{0}$ the composition of $\pi\left(v_{0}, v_{1}\right)$ and $\tau_{1}$.

Since the periodic structure is singular of type (a), the cardinality $n$ of the set $C^{(2)}$ is greater than or equal to 2 and there exists an element $\mathfrak{c} \in C^{(2)}$ so that the image $\pi\left(v_{0}, v_{1}\right)\left(h^{(0)}(\mathfrak{c})\right)$ in $G_{R\left(\Omega_{1}\right)}$ is nontrivial. Let $w\left(y^{(1)}\right)=w\left(\varphi_{0}\left(h^{(0)}(\mathfrak{c})\right)\right)$ be the image of $h^{(0)}(\mathfrak{c})$ in $\mathfrak{T}_{1}$, and consider the word $w\left(x^{(1)}\right)$ in $\mathbb{H}_{1}$. Set $\mathbb{K}$ to be $\mathbb{A}_{\mathbb{H}_{1}}\left(w\left(x^{(1)}\right)\right)$. Let $x^{(0)}=\left\{x_{1}^{(0)}, \ldots, x_{n}^{(0)}\right\}$, where $n=\left|C^{(2)}\right|$. Define the graph $\Gamma_{0}$ as follows. If the canonical parabolic subgroup $\mathbb{K}^{\perp}$ is nonabelian, set

- $V\left(\Gamma_{0}\right)=V\left(\Gamma_{1}\right) \cup\left\{x_{1}^{(0)}, \ldots, x_{n}^{(0)}\right\}$,

- $\left.E_{c}\left(\Gamma_{0}\right)=E_{c}\left(\Gamma_{1}\right) \cup\left\{\left(x_{i}^{(0)}, x_{j}^{(0)}\right) \mid 1 \leq i<j \leq n\right)\right\}$,

- $E_{d}\left(\Gamma_{0}\right)=E_{d}\left(\Gamma_{1}\right) \cup\left\{\left(x_{i}^{(0)}, x_{j}^{(1)}\right) \mid\right.$ for all $\left.x_{j}^{(1)} \in \mathbb{K}, i=1, \ldots, n\right\}$. 
If the canonical parabolic subgroup $\mathbb{K}^{\perp}$ is abelian, set

- $V\left(\Gamma_{0}\right)=V\left(\Gamma_{1}\right) \cup\left\{x_{1}^{(0)}, \ldots, x_{n}^{(0)}\right\}$;

- $E_{c}\left(\Gamma_{0}\right)=E_{c}\left(\Gamma_{1}\right) \cup\left\{\left(x_{i}^{(0)}, x_{j}^{(0)}\right) \mid 1 \leq i<j \leq n\right\} \cup\left\{\left(x_{i}^{(0)}, x_{j}^{(1)}\right) \mid x_{j}^{(1)} \in \mathbb{K}^{\perp}, 1 \leq\right.$ $i \leq n\}$;

- $E_{d}\left(\Gamma_{0}\right)=E_{d}\left(\Gamma_{1}\right) \cup\left\{\left(x_{i}^{(0)}, x_{j}^{(1)}\right) \mid\right.$ for all $\left.x_{j}^{(1)} \in \mathbb{K}, i=1, \ldots, n\right\}$.

Then the group $\mathbb{H}_{0}$ is defined to be $\mathbb{H}\left(\Gamma_{0}\right)$. Let $S_{0}^{\prime}$ be the set of relations

$$
\left[x_{i}^{(0)}, C_{\mathfrak{T}_{1}}\left(\varphi_{0}\left(h^{(0)}(\mathfrak{c})\right)\right)\right]=1, \quad \text { for all } 1 \leq i \leq n,
$$

and set $\mathfrak{T}_{0}$ to be the quotient $\mathbb{H}_{0} / S_{0}$, where $S_{0}=S_{1} \cup S_{0}^{\prime}$.

Recall that, by definition of the generating set $\bar{x}$ associated to the periodic structure and since the generalised equation is periodised (the periodic structure being singular), the set $\{h(e) \mid e \in T\} \cup\left\{h^{(0)}\left(C^{(1)}\right), h^{(0)}\left(C^{(2)}\right)\right\}$ is a basis of the free group $F\left(\left\{h_{k}^{(0)} \in\right.\right.$ $\sigma, \sigma \in \mathcal{P}\})$. Furthermore, any $h_{k}^{(0)} \in \sigma, \sigma \in \mathcal{P}$ such that $h_{k}^{(0)}=h(e), e \notin T, e: v \rightarrow v^{\prime}$, is expressed in the generating set $\{h(e) \mid e \in T\} \cup\left\{h^{(0)}\left(C^{(1)}\right), h^{(0)}\left(C^{(2)}\right)\right\}$ as

$$
h_{k}^{(0)}=h^{(0)}\left(\mathfrak{p}\left(v_{0}, v\right)\right)^{-1} v_{k 1}\left(C^{(1)}\right) v_{k 2}\left(C^{(2)}\right) h^{(0)}\left(\mathfrak{p}\left(v_{0}, v^{\prime}\right)\right),
$$

where

$$
\begin{gathered}
v_{k 1}\left(C^{(1)}\right) \in\left\langle h^{(0)}\left(C^{(1)}\right)\right\rangle, v_{k 2}\left(C^{(2)}\right) \in\left\langle h^{(0)}\left(C^{(2)}\right)\right\rangle, \\
\mathfrak{p}\left(v_{0}, v\right), \mathfrak{p}\left(v_{0}, v^{\prime}\right) \in\langle h(e) \mid e \in T\rangle .
\end{gathered}
$$

Lemma 6.14 The map $\tau_{0}$,

$h_{k}^{(0)} \mapsto\left\{\begin{array}{lr}\varphi_{0}\left(h_{k}^{(0)}\right) & \text { for all } h_{k}^{(0)} \in \sigma, \sigma \notin \mathcal{P}, \\ \varphi_{0}\left(h_{k}^{(0)}\right) & \text { for all } h_{k}^{(0)}=h(e), e \in T, \\ \varphi_{0}\left(h^{(0)}\left(\mathfrak{p}\left(v_{0}, v\right)\right)^{-1}\right) \varphi_{0}\left(v_{k 1}\left(C^{(1)}\right)\right) v_{k 2}\left(x^{(0)}\right) & \\ \varphi_{0}\left(h^{(0)}\left(\mathfrak{p}\left(v_{0}, v^{\prime}\right)\right)\right) & \text { for all } h_{k}^{(0)}=h(e), e \notin T,\end{array}\right.$

extends to a homomorphism from $G_{\Omega_{0}}$ to $\mathfrak{T}_{0}$.

Proof By [14, Lemma 6.14], the set

$$
\left\{h_{k}^{(0)} \mid h_{k}^{(0)} \in \sigma, \sigma \notin \mathcal{P}\right\} \cup\left\{h_{k}^{(0)} \mid h_{k}^{(0)} \in T\right\} \cup h^{(0)}\left(C^{(1)} \cup C^{(2)}\right)
$$

is a generating set of $G_{\Omega_{0}}$.

Notice that, by definition, the map $\tau_{0}$ on the set

$$
S=\left\{h_{k}^{(0)} \mid h_{k}^{(0)} \in \sigma, \sigma \notin \mathcal{P}\right\} \cup\left\{h_{k}^{(0)} \mid h_{k}^{(0)} \in T\right\} \cup h^{(0)}\left(C^{(1)}\right),
$$


coincides with the homomorphism $\varphi_{0}$. It follows that $\tau_{0}$ extends to a homomorphism on the subgroup $\langle S\rangle$ of $G_{\Omega_{0}}$.

We have that, by [14, Lemma 6.14], since the generalised equation is periodised, that is, $\left[h^{(0)}\left(\mathfrak{c}_{1}\right), h^{(0)}\left(\mathfrak{c}_{2}\right)\right]=1$ for all cycles $\mathfrak{c}_{1}, \mathfrak{c}_{2} \in C^{(1)} \cup C^{(2)}$, all the relations $h^{(0)}(\mu)^{-\varepsilon(\mu)} h^{(0)}(\Delta(\mu))^{\varepsilon(\Delta(\mu))}$ belong to the subgroup generated by $S$ and thus $\tau_{0}\left(h^{(0)}(\mu)^{-\varepsilon(\mu)} h^{(0)}(\Delta(\mu))^{\varepsilon(\Delta(\mu))}\right)=1$.

Therefore, in order to show that the map $\tau_{0}$ induces a homomorphism from $G \Upsilon_{0}$ to $\mathfrak{T}_{0}$ we need to show that $\tau_{0}\left(\left[h^{(0)}\left(\mathfrak{c}_{1}\right), h^{(0)}\left(\mathfrak{c}_{2}\right)\right]\right)=1$ in $\mathfrak{T}_{0}$ for all $\mathfrak{c}_{1}, \mathfrak{c}_{2} \in C^{(1)} \cup C^{(2)}$. If $\mathfrak{c}_{1}, \mathfrak{c}_{2} \in C^{(1)}$, then

$$
\tau_{0}\left(\left[h^{(0)}\left(\mathfrak{c}_{1}\right), h^{(0)}\left(\mathfrak{c}_{2}\right)\right]\right)=\varphi_{0}\left(\left[h^{(0)}\left(\mathfrak{c}_{1}\right), h^{(0)}\left(\mathfrak{c}_{2}\right)\right]\right)=1 .
$$

If $\mathfrak{c}_{1}, \mathfrak{c}_{2} \in C^{(2)}$, then, by definition of $\Gamma_{0}$,

$$
\tau_{0}\left(\left[h^{(0)}\left(\mathfrak{c}_{1}\right), h^{(0)}\left(\mathfrak{c}_{2}\right)\right]\right)=\left[x_{i}^{(0)}, x_{j}^{(0)}\right]=1 .
$$

Finally, assume that $\mathfrak{c}_{1} \in C^{(1)}$ and $\mathfrak{c}_{2} \in C^{(2)}$. Since $\left[h^{(0)}\left(\mathfrak{c}_{1}\right), h^{(0)}(\mathfrak{c})\right]=1$ in $G_{\Omega_{0}}$, for all $\mathfrak{c}_{1} \in C^{(1)}$, and the nontrivial cycle $\mathfrak{c} \in C^{(2)}$ that defines the subgroup $\mathbb{K}$, we have that $\left[\varphi_{0}\left(h^{(0)}\left(\mathfrak{c}_{1}\right)\right), \varphi_{0}\left(h^{(0)}(\mathfrak{c})\right)\right]=1$ in $\mathfrak{T}_{1}$ and so $\varphi_{0}\left(h^{(0)}\left(\mathfrak{c}_{1}\right)\right) \in C_{\mathfrak{T}_{1}}\left(\varphi_{0}\left(h^{(0)}(\mathfrak{c})\right)\right.$. We conclude from the definition of $S_{0}$ that $\tau_{0}\left(\left[h^{(0)}\left(\mathfrak{c}_{1}\right), h^{(0)}\left(\mathfrak{c}_{2}\right)\right]\right)=1$ in $\mathfrak{T}_{0}$.

To prove that $\tau_{0}$ extends to a homomorphism from $G_{\Omega_{0}}$ to $\mathfrak{T}_{0}$ it is left to show that $\tau_{0}\left(\left[h_{i}^{(0)}, h_{j}^{(0)}\right]\right)=1$, for all $h_{i}^{(0)}, h_{j}^{(0)}$ such that $\Re \Upsilon_{0}\left(h_{i}^{(0)}, h_{j}^{(0)}\right)$. In fact, since the map extends to a homomorphism on the subgroup generated by $S$, we only need to check that we have $\tau_{0}\left(\left[h_{i}^{(0)}, h_{j}^{(0)}\right]\right)=1$, for all $h_{j}^{(0)} \in \mathcal{P}$ so that $\Re \Upsilon_{0}\left(h_{i}^{(0)}, h_{j}^{(0)}\right)$. For every $P$ periodic solution $H^{(0)}$, we have that $\operatorname{alph}\left(H_{i}^{(0)}\right) \subset \operatorname{alph}\left(H_{j}^{(0)}\right)$, for all $h_{i}^{(0)} \in \sigma, \sigma \in \mathcal{P}$, $h_{j}^{(0)} \in \mathcal{P}$. Recall that if $\Re \Upsilon_{0}\left(h_{i}^{(0)}, h_{j}^{(0)}\right)$, then for all solutions $H^{(0)}$ of $\Omega_{0}$ we have that $H_{i}^{(0)} \leftrightarrows H_{j}^{(0)}$. Therefore, if $\Re_{\Upsilon_{0}}\left(h_{i}^{(0)}, h_{j}^{(0)}\right)$ and $h_{j}^{(0)} \in \mathcal{P}$, then $h_{i}^{(0)} \in \sigma, \sigma \notin \mathcal{P}$.

Furthermore, since the periodic structure is not strongly singular and the set $\Re \Upsilon_{0}$ is completed, it follows that for all $h_{j}^{(0)} \in \mathcal{P}$ and for every $h_{i}^{(0)}$ such that $\Re \Upsilon_{0}\left(h_{i}^{(0)}, h_{j}^{(0)}\right)$, one has that $\Re \Upsilon_{0}\left(h_{i}^{(0)}, h_{k}^{(0)}\right)$, for all $h_{k}^{(0)} \in \sigma, \sigma \in \mathcal{P}$.

By the properties of the elementary transformations, $\Re_{\Upsilon_{1}}\left(h_{r}^{(1)}, h_{s}^{(1)}\right)$ for all $h_{r}^{(1)}$ from $\pi\left(v_{0}, v_{1}\right)\left(h_{i}^{(0)}\right)$ and all $h_{s}^{(1)} \in \pi\left(v_{0}, v_{1}\right)\left(h_{k}^{(0)}\right), h_{k}^{(0)} \in \sigma, \sigma \in \mathcal{P}$. By the induction hypothesis (IH2) on $\tau_{1}$, we have $\mathbb{A}\left(H^{(1)^{\prime}}\left(y_{m}^{(1)}\right)\right) \supset \mathbb{A}\left(H_{i}^{(0)}\right)$ and $\mathbb{A}\left(H^{(1)^{\prime}}\left(y_{n}^{(1)}\right)\right) \supset$ $\mathbb{A}\left(H_{k}^{(0)}\right)$ for any $y_{m}^{(1)}$ in $\varphi_{0}\left(h_{i}^{(0)}\right), y_{n}^{(1)}$ in $\varphi_{0}\left(h_{k}^{(0)}\right)$ and any solution $H^{(1)}$ of $\Omega_{1}$ that factors through the branch, hence

$$
H^{(1) \prime}\left(y_{m}^{(1)}\right) \leftrightarrows H^{(1) \prime}\left(y_{n}^{(1)}\right) .
$$

By the induction hypothesis (IH1) on $\mathbb{H}_{1}$, we have that $\left(x_{n}^{(1)}, x_{m}^{(1)}\right) \in E_{d}\left(\Gamma_{1}\right)$ and so $\varphi_{0}\left(h_{i}^{(0)}\right) \leftrightarrows \varphi_{0}\left(h_{k}^{(0)}\right)$ in $\mathbb{H}_{1}$, for all $h_{k}^{(0)} \in \sigma, \sigma \in \mathcal{P}$ and, hence, in particular, $\varphi_{0}\left(h_{i}^{(0)}\right)$ 
belongs to the subgroup $\mathbb{K}$ of $\mathbb{H}_{1}$. Therefore, if $\Re \Upsilon_{0}\left(h_{i}^{(0)}, h_{j}^{(0)}\right)$ and $h_{j}^{(0)} \in \mathcal{P}$, then $\tau_{0}\left(\left[h_{i}^{(0)}, h_{j}^{(0)}\right]\right)=1$.

Lemma 6.15 The homomorphism $\tau_{0}: G_{\Omega_{0}} \rightarrow \mathfrak{T}_{0}$ makes diagram (11) commutative.

Proof By [14, Lemma 6.18], for any solution $H^{(0)}$ that factors through the branch, there exist a solution $H^{(1)}$ of $\Omega_{1}$ and an automorphism $\phi$ from the group $\mathfrak{A}\left(\Omega_{0}\right)$ (see [14, Definition 6.16]) such that $H^{(0)}=\phi \pi\left(v_{0}, v_{1}\right) H^{(1)}$. Furthermore, if

$$
S=\left\{h_{k}^{(0)} \mid h_{k}^{(0)} \in \sigma, \sigma \notin \mathcal{P}\right\} \cup\left\{h_{k}^{(0)} \mid h_{k}^{(0)} \in T\right\} \cup h^{(0)}\left(C^{(1)}\right),
$$

then we have that $H^{(0)}\left(h_{i}^{(0)}\right)=H^{(1)}\left(\pi\left(v_{0}, v_{1}\right)\left(h_{i}^{(0)}\right)\right)$, where $h_{i}^{(0)} \in\langle S\rangle$. By the induction hypothesis, there exists a homomorphism $H^{(1)^{\prime}}$ from $\mathfrak{T}_{1}$ to $\mathbb{G}$ that makes diagram (11) commutative. We define $H^{(0)^{\prime}}$ on the subgroup $\mathfrak{T}_{1}$ of $\mathfrak{T}_{0}$ to be $H^{(1)^{\prime}}$ and we define $H^{(0)^{\prime}}\left(y_{i}^{(0)}\right)=H^{(0)}\left(\mathfrak{c}_{2 i}\right)$, where $\mathfrak{c}_{2 i} \in C^{(2)}$ is so that $\tau_{0}\left(h^{(0)}\left(\mathfrak{c}_{2 i}\right)\right)=y_{i}^{(0)}$.

If we show that $H^{(0)^{\prime}}$ is a homomorphism, then the commutativity of the diagram follows by construction. Since all solutions are $P$-periodic, it follows that

$$
H^{(0)^{\prime}}\left(y_{i}^{(0)}\right)=H^{(0)}\left(\mathfrak{c}_{2}\right)=P^{n} \quad \text { and } \quad H^{(0)^{\prime}}\left(\varphi_{0}\left(h^{(0)}(\mathfrak{c})\right)\right)=P^{k}, \quad k \neq 0 .
$$

Therefore, $H^{(0)^{\prime}}$ is a homomorphism and it makes diagram (11) commutative.

Lemma 6.16 The subgroup $\mathbb{K}=\mathbb{A}\left(w\left(x^{(1)}\right)\right)$ of $\mathbb{H}_{1}$ is $E_{d}\left(\Gamma_{1}\right)$-coirreducible.

Proof Since items of generalised equations have associated parabolic subgroups, there exists a canonical parabolic subgroup $\mathbb{G}_{\mathbb{K}}<\mathbb{G}$ such that for any solution that factors through the branch we have that $\left\langle\operatorname{alph}\left(H^{(0)^{\prime}}(\mathbb{K})\right)\right\rangle=\mathbb{G}_{\mathbb{K}}$. By definition of $\mathbb{K}$ and by the induction hypothesis (IH1), we have that $H^{(1)^{\prime}}\left(w\left(x^{(1)}\right)\right) \leftrightarrows \mathbb{G}_{\mathbb{K}}$. Furthermore, if $a_{i} \leftrightarrows$ $H^{(1)^{\prime}}\left(w\left(x^{(1)}\right)\right)$, where $a \in \mathcal{A}, \mathbb{G}=\mathbb{G}(\mathcal{A})$, again, by the induction hypothesis (IH1), we have that $a_{i} \in \mathbb{K}$ and so $a_{i} \in \mathbb{G}_{\mathbb{K}}$, hence we have that $\left\langle\operatorname{alph}\left(H^{(1)^{\prime}}\left(w\left(x^{(1)}\right)\right)\right)^{\perp}\right\rangle=\mathbb{G}_{\mathbb{K}}$.

Let us now address the statement of the lemma. By definition the group $\mathbb{K}$ is closed, ie $\mathbb{K}=\mathbb{K}^{\perp \perp}$. We only need to show that $\mathbb{K}^{\perp}$ is $E_{d}\left(\Gamma_{1}\right)$-directly indecomposable.

Assume the contrary, then $\mathbb{K}^{\perp}=\mathbb{K}_{1} \times \cdots \times \mathbb{K}_{r}$, where $r>1$ (with respect to the edges from $\left.E_{d}\left(\Gamma_{1}\right)\right)$. Without loss of generality, we may assume that $r=2$. Since items of generalised equations have associated parabolic subgroups, there exist canonical parabolic subgroups $\mathbb{G}_{\mathbb{K}_{i}}<\mathbb{G}, i=1,2$, such that for solutions $H^{(0)}$ that factor through the branch we have $H^{(0)^{\prime}}\left(\mathbb{K}_{i}\right)=\mathbb{G}_{\mathbb{K}_{i}}, i=1,2$. Furthermore, by the induction hypothesis (IH1), one has that $\mathbb{G}_{\mathbb{K}_{1}} \leftrightarrows \mathbb{G}_{\mathbb{K}_{2}}$. 
Since we have that $\left\langle\operatorname{alph}\left(H^{(1)^{\prime}}\left(w\left(x^{(1)}\right)\right)\right)^{\perp}\right\rangle=\mathbb{G}_{\mathbb{K}}$, it follows that $H^{(1)^{\prime}}\left(w\left(x^{(1)}\right)\right) \in$ $\mathbb{G}_{\mathbb{K}}^{\perp}<\mathbb{G}_{\mathbb{K}_{1}} \times \mathbb{G}_{\mathbb{K}_{2}}$. Furthermore, it follows that $H^{(1)^{\prime}}\left(w\left(x^{(1)}\right)\right)$ contains at least one $\mathbb{G}_{\mathbb{K}_{1}} \times \mathbb{G}_{\mathbb{K}_{2}}$-alternation.

Since every solution is $P$-periodic, it follows that $H^{(1)^{\prime}}\left(w\left(x^{(1)}\right)\right)=P^{n}$ and $P$ contains at least one $\mathbb{G}_{\mathbb{K}_{1}} \times \mathbb{G}_{\mathbb{K}_{2}}$-alternation. By the description of solutions of generalised equations periodised with respect to a periodic structure (see [14, Chapter 6]) for every $m \in \mathbb{N}$, there exists a solution $H^{(0)}$ of $\Omega_{0}$ so that $H^{(0)}\left(\mathfrak{c}_{2}\right) \doteq P^{m^{\prime}}$, where $m^{\prime} \geq m$, $\mathfrak{c}_{2} \in C^{(2)}$. Hence, $H^{(0)}\left(\mathfrak{c}_{2}\right)$ contains at least $m^{\prime}$ many $\mathbb{G}_{\mathbb{K}_{1}} \times \mathbb{G}_{\mathbb{K}_{2}}$-alternations. This derives a contradiction, since, by Remark $6.1, H^{(0)}\left(\mathfrak{c}_{2}\right)$ is a subword of a word in the normal form and, by Lemma 2.3 , it contains only a bounded number of alternations. Hence, we conclude that $P$ is a cyclically reduced irreducible root element and so $r=1$ and $\mathbb{K}^{\perp}$ is $E_{d}\left(\Gamma_{1}\right)$-directly indecomposable.

Remark 6.17 Solutions of generalised equations define graphical equalities, so if solutions that factor through the branch are $P$-periodic, then the period $P$ is cyclically reduced. Furthermore, as we have shown above, the period $P$ is an irreducible root element from $\mathbb{G}_{\mathbb{K}}{ }^{\perp}=\mathbb{G}_{\mathbb{K}}^{\perp}$.

Lemma 6.18 The group $\mathbb{H}_{0}$ satisfies the induction hypothesis (IH1): for every $x_{i}, x_{j} \in \mathbb{H}_{0}$, we have that $\left(x_{i}, x_{j}\right) \in E_{d}\left(\Gamma_{0}\right)$ if and only if $H^{\prime}\left(x_{i}\right) \leftrightarrows H^{\prime}\left(x_{j}\right)$ for all homomorphisms $H^{\prime}$ induced by solutions $H$ that factor through the branch; furthermore, we have that if $\left(x_{i}, x_{j}\right) \in E_{c}\left(\Gamma_{0}\right)$, then $H^{\prime}\left(x_{i}\right)$ and $H^{\prime}\left(x_{j}\right)$ belong to the same cyclic subgroup for all homomorphisms $H^{\prime}$ induced by solutions $H$ that factor through the branch.

Proof If $x_{i}, x_{j} \in \mathbb{H}_{1}<\mathbb{H}_{0}$, then the statement follows by the induction hypothesis on $\mathbb{H}_{1}$.

By definition, $\left(x_{i}^{(0)}, x_{j}^{(0)}\right) \in E_{c}\left(\Gamma_{0}\right)$ for all $1 \leq i<j \leq n$. Let us show that $H^{(0) \prime}\left(x_{i}^{(0)}\right)$ and $H^{(0) '}\left(x_{j}^{(0)}\right)$ belong to the same cyclic subgroup. Indeed, since solutions that factor through the branch are $P$-periodic, it follows that $H^{(0)^{\prime}}\left(x_{i}^{(0)}\right)=H^{(0)}\left(\mathfrak{c}_{2 i}\right)=P^{k}$ for all $i=1, \ldots, n$.

Furthermore, if $\mathbb{K}^{\perp}$ is abelian, then $\left(x_{i}^{(0)}, x_{j}^{(1)}\right) \in E_{c}\left(\Gamma_{0}\right)$ for all $x_{j}^{(1)} \in \mathbb{K}^{\perp}$. By the induction hypothesis (IH1), for all solutions the subgroup $\left\langle H^{(0)^{\prime}}\left(\mathbb{K}^{\perp}\right)\right\rangle=\left\langle H^{(1)^{\prime}}\left(\mathbb{K}^{\perp}\right)\right\rangle$ is a cyclic subgroup of $\mathbb{G}$. Since, by Remark 6.17 , the period $P$ is an irreducible element from $\left\langle\operatorname{alph}\left(H^{(0) \prime}\left(\mathbb{K}^{\perp}\right)\right)\right\rangle$, it follows that $H^{(0)^{\prime}}\left(x_{i}^{(0)}\right)=H^{(0)}\left(\mathfrak{c}_{2 i}\right)=P^{k}$ and $H^{(0) \prime}\left(x_{j}^{(1)}\right)$ belong to the same cyclic subgroup. 
By definition $\left(x_{i}^{(0)}, x_{j}^{(1)}\right) \in E_{d}\left(\Gamma_{0}\right)$, for all $x_{j}^{(1)} \in \mathbb{K}, i=1, \ldots, n$. From the definition of $\mathbb{K}$ and the induction hypothesis on the tower $\left(\mathfrak{T}_{1}, \mathbb{H}_{1}\right)$, we have that

$$
H^{(1) \prime}\left(\varphi_{0}(\mathfrak{c})\right)=H^{(1)}\left(\pi\left(v_{0}, v_{1}\right)(\mathfrak{c})\right)=P^{k} \leftrightarrows H^{(1)^{\prime}}(\mathbb{K})
$$

Since solutions are $P$-periodic, it follows that

$$
H^{(0)^{\prime}}\left(x_{i}^{(0)}\right)=H^{(0)}\left(\mathfrak{c}_{2 i}\right)=P^{l} \leftrightarrows H^{(1)^{\prime}}(\mathbb{K})=H^{(0)^{\prime}}(\mathbb{K})
$$

where $\tau_{0}\left(\mathfrak{c}_{2 i}\right)=x_{i}^{(0)}, i=1, \ldots,\left|C^{(2)}\right|=n$.

Assume that for $x_{j}^{(1)} \in \mathbb{H}_{1}$ we have $H^{(0)^{\prime}}\left(x_{j}^{(1)}\right) \leftrightarrows H^{(0)^{\prime}}\left(x_{i}^{(0)}\right)$ for some $i=1, \ldots, n$. Since $H^{(0) \prime}\left(x_{i}^{(0)}\right)=H^{(0)}\left(\mathfrak{c}_{2 i}\right)=P^{l}$, it follows that

$$
\begin{aligned}
H^{(0)^{\prime}}\left(x_{j}^{(1)}\right)= & H^{(1)^{\prime}}\left(x_{j}^{(1)}\right) \\
& \leftrightarrows H^{(0)}\left(\pi\left(v_{0}, v_{1}\right)(h(\mathfrak{c}))\right)=H^{(0)^{\prime}}\left(\varphi_{0}(h(\mathfrak{c}))\right)=H^{(1)^{\prime}}(\varphi(h(\mathfrak{c})))=P^{l}
\end{aligned}
$$

By the induction hypothesis $(\mathrm{IH} 1)$ on $\mathbb{H}_{1}$, it follows that $x_{j}^{(1)} \in \mathbb{A}\left(\varphi_{0}(h(\mathfrak{c}))\right)=\mathbb{K}$. Therefore, $\left(x_{j}^{(1)}, x_{i}^{(0)}\right) \in E_{d}\left(\Gamma_{0}\right)$.

Lemma 6.19 The homomorphism $\tau_{0}$ satisfies the induction hypothesis (IH2): for all $h_{i}^{(0)}$, if $\tau_{0}\left(h_{i}^{(0)}\right)=x_{i 1} \cdots x_{i k}$, then we have that $\mathbb{A}\left(H^{\prime}\left(x_{i j}\right)\right) \supset \mathbb{A}\left(H_{i}^{0}\right)$ and $\bigcap_{j=1}^{k} \mathbb{A}\left(H^{\prime}\left(x_{i j}\right)\right)=\mathbb{A}\left(H_{i}^{(0)}\right)$ for solutions that factor through the branch.

Proof Recall that if

$$
S=\left\{h_{k}^{(0)} \mid h_{k}^{(0)} \in \sigma, \sigma \notin \mathcal{P}\right\} \cup\left\{h_{k}^{(0)} \mid h_{k}^{(0)} \in T\right\} \cup h^{(0)}\left(C^{(1)}\right),
$$

then, for $h_{i}^{(0)} \in\langle S\rangle$ and for all solutions that factor through the branch $H^{(0)}$ we have that $H^{(0)}\left(h_{i}^{(0)}\right)=H^{(1)}\left(\pi\left(v_{0}, v_{1}\right)\left(h_{i}^{(0)}\right)\right)$.

If $h_{i}^{(0)} \in\langle S\rangle$, then $\tau_{0}\left(h_{i}^{(0)}\right)=\tau_{1} \pi\left(v_{0}, v_{1}\right)\left(h_{i}^{(0)}\right)$. If $\pi\left(v_{0}, v_{1}\right)\left(h_{i}^{(0)}\right)=h_{i_{1}}^{(1)} \cdots h_{i_{m}}^{(1)}$, then since solutions of the generalised equations define graphical equalities it follows that for every solution $H^{(1)}$ that factors through the branch, we have that $H^{(1)}\left(\pi\left(v_{0}, v_{1}\right)\left(h_{i}^{(0)}\right)\right)=H_{i_{1}}^{(1)} \cdots H_{i_{m}}^{(1)}$ and so

$$
\mathbb{A}\left(H^{(1)}\left(h_{i_{j}}^{(1)}\right)\right) \supset \mathbb{A}\left(H^{(1)}\left(\pi\left(v_{0}, v_{1}\right)\left(h_{i}^{(0)}\right)\right)\right)=\mathbb{A}\left(H_{i}^{(0)}\right) .
$$

Since, by induction assumption, the statement of the lemma holds for $\tau_{1}$, we conclude that the statement holds for $\tau_{0}\left(h_{i}^{(0)}\right)=\tau_{1} \pi\left(v_{0}, v_{1}\right)\left(h_{i}^{(0)}\right)$, for all $h_{i}^{(0)} \in\langle S\rangle$.

If $h_{k}^{(0)} \notin\langle S\rangle$, then $h_{k}^{(0)} \in \mathcal{P}$ and

$$
h_{k}^{(0)}=h^{(0)}\left(\mathfrak{p}\left(v_{0}, v\right)\right) v_{k 1}\left(C^{(1)}\right) v_{k 2}\left(C^{(2)}\right) h^{(0)}\left(\mathfrak{p}\left(v_{0}, v^{\prime}\right)\right)
$$


for some

$$
\begin{gathered}
v_{k 1}\left(C^{(1)}\right) \in\left\langle h^{(0)}\left(C^{(1)}\right)\right\rangle, \quad v_{k 2}\left(C^{(2)}\right) \in\left\langle h^{(0)}\left(C^{(2)}\right)\right\rangle, \\
h^{(0)}\left(\mathfrak{p}\left(v_{0}, v\right)\right), h^{(0)}\left(\mathfrak{p}\left(v_{0}, v^{\prime}\right)\right) \in\langle h(e) \mid e \in T\rangle .
\end{gathered}
$$

In this case,

$$
\tau_{0}\left(h_{k}^{(0)}\right)=\varphi_{0}\left(h^{(0)}\left(\mathfrak{p}\left(v_{0}, v\right)\right) v_{k 1}\left(C^{(1)}\right)\right) v_{k 2}\left(x^{(0)}\right) \varphi_{0}\left(h^{(0)}\left(\mathfrak{p}\left(v_{0}, v^{\prime}\right)\right) .\right.
$$

Since every solution $H^{(0)}$ that factors through the branch is $P$-periodic, it follows that $\operatorname{alph}\left(H_{i}^{(0)}\right) \subset \operatorname{alph}(P)$, for all $h_{i}^{(0)} \in \sigma, \sigma \in \mathcal{P}$. Hence, for all $x_{i}$ in the words $\varphi_{0}\left(h^{(0)}\left(\mathfrak{p}\left(v_{0}, v\right)\right) v_{k 1}\left(C^{(1)}\right)\right)$ and $\varphi_{0}\left(h^{(0)}\left(\mathfrak{p}\left(v_{0}, v^{\prime}\right)\right)\right)$, by the induction hypothesis (IH2) on $\tau_{1}$, we can conclude that

$$
\mathbb{A}\left(H^{(0)^{\prime}}\left(x_{i}\right)\right) \supset \mathbb{A}(P)=\mathbb{A}\left(H_{k}^{(0)}\right) .
$$

Furthermore,

$$
\operatorname{alph}\left(H^{(0)^{\prime}}\left(x_{i}^{(0)}\right)\right)=\operatorname{alph}\left(H^{(0)}\left(\mathfrak{c}_{2 i}\right)\right)=\operatorname{alph}\left(P^{k}\right)=\operatorname{alph}\left(H_{k}^{(0)}\right)
$$

and so $\mathbb{A}\left(H^{(0)^{\prime}}\left(x_{i}^{(0)}\right)\right)=\mathbb{A}\left(H_{k}^{(0)}\right)$ and, in particular,

$$
\bigcap_{x_{i} \text { in } \tau_{0}\left(h_{k}^{(0)}\right)} \mathbb{A}\left(H^{(0)^{\prime}}\left(x_{i}\right)\right)=\mathbb{A}\left(H_{k}^{(0)}\right) .
$$

Assume now that the periodic structure is singular of type (b), ie the set $C^{(2)}$ has exactly one element and there exists a cycle $\mathfrak{c}=\mathfrak{c}_{e_{0}} \in C^{(1)}, h^{(0)}\left(e_{0}\right) \notin \mathcal{P}$ such that $h^{(0)}\left(\mathfrak{c}_{e_{0}}\right)=$ $w\left(h^{(0)}\right) \neq 1$ in $G_{\Omega_{0}}$. Since $\mathfrak{c} \in C^{(1)}$, for all solutions $H^{(0)}=\varphi \pi\left(v_{0}, v_{1}\right) H^{(1)}$ of $\Omega_{0}$ we have that $H^{(0)}(\mathfrak{c})=H^{(1)}\left(\pi\left(v_{0}, v_{1}\right)(h(\mathfrak{c}))\right)$. Since $G_{R\left(\Omega_{0}\right)}$ is separated by $\mathbb{G}$, there exists $H^{(0)}$ so that $1 \neq H^{(0)}(\mathfrak{c})=H^{(1)}\left(\pi\left(v_{0}, v_{1}\right)(h(\mathfrak{c}))\right)$, hence $\pi\left(v_{0}, v_{1}\right)(h(\mathfrak{c})) \neq 1$ in $G_{R\left(\Omega_{1}\right)}$. Let $w\left(x^{\prime}\right)=\varphi_{0}\left(h^{(0)}\left(\mathfrak{c}_{e_{0}}\right)\right)$ be the image of $h^{(0)}\left(\mathfrak{c}_{e_{0}}\right)$ in $\mathfrak{T}_{1}$, and consider the word $w\left(x^{(1)}\right)$ in $\mathbb{H}_{1}$. Set $\mathbb{K}$ to be $\mathbb{A}_{\mathbb{H}_{1}}\left(w\left(x^{(1)}\right)\right)$.

Define the graph $\Gamma_{0}$ as follows. If the canonical parabolic subgroup $\mathbb{K}^{\perp}$ is nonabelian, set

- $V\left(\Gamma_{0}\right)=V\left(\Gamma_{1}\right) \cup\left\{x^{(0)}\right\}$;

- $E_{c}\left(\Gamma_{0}\right)=E_{c}\left(\Gamma_{1}\right)$;

- $E_{d}\left(\Gamma_{0}\right)=E_{d}\left(\Gamma_{1}\right) \cup\left\{\left(x^{(0)}, x_{j}^{(1)}\right) \mid\right.$ for all $\left.x_{j}^{(1)} \in \mathbb{K}\right\}$.

If the canonical parabolic subgroup $\mathbb{K}^{\perp}$ is abelian, set

- $V\left(\Gamma_{0}\right)=V\left(\Gamma_{1}\right) \cup\left\{x^{(0)}\right\} ;$ 
- $E_{c}\left(\Gamma_{0}\right)=E_{c}\left(\Gamma_{1}\right) \cup\left\{\left(x^{(0)}, x_{j}^{(0)}\right) \mid x_{j}^{(1)} \in \mathbb{K}^{\perp}\right\}$;

- $E_{d}\left(\Gamma_{0}\right)=E_{d}\left(\Gamma_{1}\right) \cup\left\{\left(x^{(0)}, x_{j}^{(1)}\right) \mid\right.$ for all $\left.x_{j}^{(1)} \in \mathbb{K}\right\}$.

Now, the group $\mathbb{H}_{0}$ is defined to be $\mathbb{H}\left(\Gamma_{0}\right)$.

Let $S_{0}^{\prime}$ be the set of relations

$$
\left[x^{(0)}, C_{\mathfrak{T}_{1}}\left(\varphi_{0}\left(h^{(0)}\left(\mathfrak{c}_{e_{0}}\right)\right)\right)\right]=1,
$$

and set $\mathfrak{T}_{0}$ to be the quotient $\mathbb{H}_{0} / \operatorname{ncl}\left\langle S_{0}\right\rangle$, where $S_{0}=S_{1} \cup S_{0}^{\prime}$.

Recall that, by definition of the generating set $\bar{x}$ and since the generalised equation is periodised (the periodic structure being singular), the set $\{h(e) \mid e \in T\} \cup$ $\left\{h^{(0)}\left(C^{(1)}\right), h^{(0)}\left(C^{(2)}\right)\right\}$ is a basis of the free group on the alphabet $\left\{h_{k}^{(0)} \in \sigma, \sigma \in \mathcal{P}\right\}$. Furthermore, any $h_{k}^{(0)} \in \sigma, \sigma \in \mathcal{P}$ so that $h_{k}^{(0)}=h(e), e \notin T, e: v \rightarrow v^{\prime}$, is expressed in the generating set $\{h(e) \mid e \in T\} \cup\left\{h^{(0)}\left(C^{(1)}\right), h^{(0)}\left(C^{(2)}\right)\right\}$ as

$$
h_{k}^{(0)}=h^{(0)}\left(\mathfrak{p}\left(v_{0}, v\right)\right)^{-1} v_{k 1}\left(C^{(1)}\right) v_{k 2}\left(C^{(2)}\right) h^{(0)}\left(\mathfrak{p}\left(v_{0}, v^{\prime}\right)\right) \text {, }
$$

for some $v_{k 1}\left(C^{(1)}\right) \in\left\langle h^{(0)}\left(C^{(1)}\right)\right\rangle$ and some $v_{k 2}\left(C^{(2)}\right) \in\left\langle h^{(0)}\left(C^{(2)}\right)\right\rangle$.

Lemma 6.20 The map $\tau_{0}$,

$h_{k}^{(0)} \mapsto\left\{\begin{array}{lr}\varphi_{0}\left(h_{k}^{(0)}\right) & \text { for all } h_{k}^{(0)} \in \sigma, \sigma \notin \mathcal{P}, \\ \varphi_{0}\left(h_{k}^{(0)}\right) & \text { for all } h_{k}^{(0)}=h(e), e \in T, \\ \varphi_{0}\left(h^{(0)}\left(\mathfrak{p}\left(v_{0}, v\right)\right)^{-1}\right) \varphi_{0}\left(v_{k 1}\left(C^{(1)}\right)\right) v_{k 2}\left(x^{(0)}\right) & \\ \varphi_{0}\left(h^{(0)}\left(\mathfrak{p}\left(v_{0}, v^{\prime}\right)\right)\right), & \text { for all } h_{k}^{(0)}=h(e), e \notin T,\end{array}\right.$

extends to a homomorphism from $G_{\Omega_{0}}$ to $\mathfrak{T}_{0}$.

Proof The proof is analogous to the proof of Lemma 6.14 .

Lemma 6.21 The homomorphism $\tau_{0}: G_{\Omega_{0}} \rightarrow \mathfrak{T}_{0}$ makes diagram (11) commutative.

Proof The proof is analogous to the proof of Lemma 6.15 .

Lemma 6.22 The subgroup $\mathbb{K}=\mathbb{A}\left(w\left(x^{(1)}\right)\right)$ of $\mathbb{H}_{0}$ is $E_{d}\left(\Gamma_{0}\right)$-coirreducible.

Proof Proof is analogous to the proof of Lemma 6.16.

Lemma 6.23 The group $\mathbb{H}_{0}$ satisfies (IH1): for all $x_{i}, x_{j} \in \mathbb{H}_{0}$, we have that $\left(x_{i}, x_{j}\right) \in E_{d}\left(\Gamma_{0}\right)$ if and only if $H^{\prime}\left(x_{i}\right) \leftrightarrows H^{\prime}\left(x_{j}\right)$ for all homomorphisms $H^{\prime}$ induced by solutions $H$ that factor through the branch. Furthermore, we have that if $\left(x_{i}, x_{j}\right) \in E_{c}\left(\Gamma_{0}\right)$, then $H^{\prime}\left(x_{i}\right)$ and $H^{\prime}\left(x_{j}\right)$ belong to a cyclic subgroup for all homomorphisms $H^{\prime}$ induced by solutions $H$ that factor through the branch. 
Proof The proof is analogous to the proof of Lemma 6.18 .

Lemma 6.24 The homomorphism $\tau_{0}$ satisfies the induction hypothesis (IH2): For all $h_{i}^{(0)}$, if $\tau_{0}\left(h_{i}^{(0)}\right)=x_{i 1} \cdots x_{i k}$, then we have that

$$
\mathbb{A}\left(H^{(0)^{\prime}}\left(x_{i j}\right)\right) \supset \mathbb{A}\left(H_{i}^{(0)}\right) \text { and } \bigcap_{j=1}^{k} \mathbb{A}\left(H^{(0)^{\prime}}\left(x_{i j}\right)\right)=\mathbb{A}\left(H_{i}^{(0)}\right),
$$

for solutions that factor through the branch.

Proof The proof is analogous to the proof of Lemma 6.19.

We summarise the results of this section in the following

Proposition 6.25 Let $\Omega_{0} \rightarrow \Omega_{1} \rightarrow \cdots \rightarrow \Omega_{q}$ be a branch of a tribal solution tree and assume that $\Omega_{1}$ satisfies the induction hypotheses (IH), (IH1) and (IH2); see page 784 . If $\Omega_{0}=\langle\Upsilon, \Re \Upsilon\rangle$ is a generalised equation of type 2 , then there exists a graph tower $\left(\mathfrak{T}_{0}, \mathbb{H}_{0}\right)$ and a homomorphism $\tau_{0}$ from $G_{\Omega_{0}}$ to $\mathfrak{T}_{0}$ such that for each solution that factors through the branch, there exists a homomorphism from $\mathfrak{T}_{0}$ to $\mathbb{G}$ that makes diagram (11) commutative.

\subsection{Regular periodic structures}

By [14, Lemma 6.19], given a generalised equation $\Omega=\langle\Upsilon, \Re \Upsilon\rangle$ with no boundary connections, periodised with respect to a connected regular periodic structure $\langle\mathcal{P}, R\rangle$ and any periodic solution $H$ of $\Omega$ such that $\mathcal{P}(H, P)=\langle\mathcal{P}, R\rangle$ and so that $H$ is minimal with respect to the trivial group of automorphisms, either for all $k, 1 \leq k \leq \rho$ we have $\left|H_{k}\right| \leq 2 \rho|P|$, or there exists a cycle $\mathfrak{c} \in \pi_{1}\left(\Gamma, v_{\Gamma}\right)$ so that $H(\mathfrak{c})=P^{n}$, where $1 \leq n \leq 2 \rho$.

Suppose first that the solution that factors through the branch falls under the conditions of the first assumption, ie all the items from the periodic structure are of bounded length. Then, in a bounded number of steps $c$, all the items, bases and sections from the periodic structure will be transferred onto the sections that do not belong to the periodic structure. Let $\Omega_{c}$ be the corresponding generalised equation. Then every solution that factors through the branch is a solution of $\Omega_{c}$. In this case, the graph tower for $\Omega$, is the graph tower associated to $\Omega_{c}$.

Suppose now that solution that factors through the branch falls under the conditions of the second assumption. Then there exists a cycle $\mathfrak{c} \in \pi_{1}\left(\Gamma, v_{\Gamma}\right)$ so that $H(\mathfrak{c})=P^{n}$, where $1 \leq n \leq 2 \rho$. Let $w=\varphi_{0}(h(\mathfrak{c}))$ be the image of $h(\mathfrak{c})$ in $\mathfrak{T}_{1}, w=w\left(y^{(1)}\right)$ and consider the word $w\left(x^{(1)}\right)$ in $\mathbb{H}_{1}$. Set $\mathbb{K}$ to be $\mathbb{A}_{\mathbb{H}_{1}}\left(w\left(x^{(1)}\right)\right)$. 
Let $x^{(0)}=\left\{x_{1}^{(0)}, \ldots, x_{\mathfrak{m}}^{(0)}\right\}$. Define the graph $\Gamma_{0}$ as follows. If the canonical parabolic subgroup $\mathbb{K}^{\perp}$ is nonabelian, set

- $V\left(\Gamma_{0}\right)=V\left(\Gamma_{1}\right) \cup\left\{x_{1}^{(0)}, \ldots, x_{\mathfrak{m}}^{(0)}\right\}$;

- $\left.E_{c}\left(\Gamma_{0}\right)=E_{c}\left(\Gamma_{1}\right) \cup\left\{\left(x_{i}^{(0)}, x_{j}^{(0)}\right) \mid 1 \leq i<j \leq \mathfrak{m}\right)\right\}$;

- $E_{d}\left(\Gamma_{0}\right)=E_{d}\left(\Gamma_{1}\right) \cup\left\{\left(x_{i}^{(0)}, x_{j}^{(1)}\right) \mid\right.$ for all $\left.x_{j}^{(1)} \in \mathbb{K}, i=1, \ldots, \mathfrak{m}\right\}$.

If the canonical parabolic subgroup $\mathbb{K}^{\perp}$ is abelian, set

- $V\left(\Gamma_{0}\right)=V\left(\Gamma_{1}\right) \cup\left\{x_{1}^{(0)}, \ldots, x_{\mathfrak{m}}^{(0)}\right\}$;

- $E_{c}\left(\Gamma_{0}\right)=E_{c}\left(\Gamma_{1}\right) \cup\left\{\left(x_{i}^{(0)}, x_{j}^{(0)}\right) \mid 1 \leq i<j \leq \mathfrak{m}\right\} \cup\left\{\left(x_{i}^{(0)}, x_{j}^{(1)}\right) \mid x_{j}^{(1)} \in\right.$ $\left.\mathbb{K}^{\perp}, 1 \leq i \leq \mathfrak{m}\right\}$

- $E_{d}\left(\Gamma_{0}\right)=E_{d}\left(\Gamma_{1}\right) \cup\left\{\left(x_{i}^{(0)}, x_{j}^{(1)}\right) \mid\right.$ for all $\left.x_{j}^{(1)} \in \mathbb{K}, i=1, \ldots, \mathfrak{m}\right\}$.

Then the group $\mathbb{H}_{0}$ is defined to be $\mathbb{H}\left(\Gamma_{0}\right)$.

Let $S_{0}^{\prime}$ be the set of relations defined as follows. For every equation $u_{i e}^{h\left(e_{i}\right)}=z_{i e}$ (we use notation from [14, Chapter 6]), we write

$$
\left[x_{i}^{(0)}, C_{\mathfrak{T}_{1}}\left(\varphi_{0}\left(u_{i e}\right)\right)\right]=1, i=1, \ldots, \mathfrak{m}
$$

and set $\mathfrak{T}_{0}$ to be the quotient $\mathbb{H}_{0} / S_{0}$, where $S_{0}=S_{1} \cup S_{0}^{\prime}$.

Recall that, by definition of the generating set $\bar{x}$ associated to the periodic structure and since the generalised equation is periodised, any $h_{k}^{(0)} \in \sigma, \sigma \in \mathcal{P}$ such that $h_{k}^{(0)}=h(e), e \notin T, e: v \rightarrow v^{\prime}$ is expressed in the generating set $\{h(e) \mid$ $e \in T\} \cup\left\{h^{(0)}\left(C^{(1)}\right), h^{(0)}\left(C^{(2)}\right)\right\}$ as

$$
h_{k}^{(0)}=h^{(0)}\left(\mathfrak{p}\left(v_{0}, v\right)\right)^{-1} v_{k 1}\left(C^{(1)}\right) v_{k 2}\left(C^{(2)}\right) h^{(0)}\left(\mathfrak{p}\left(v_{0}, v^{\prime}\right)\right)
$$

for some $v_{k 1}\left(C^{(1)}\right) \in\left\langle h^{(0)}\left(C^{(1)}\right)\right\rangle$ and some $v_{k 2}\left(C^{(2)}\right) \in\left\langle h^{(0)}\left(C^{(2)}\right)\right\rangle$. Furthermore, the paths $\mathfrak{p}\left(v_{0}, v\right)$ and $\mathfrak{p}\left(v_{0}, v^{\prime}\right)$ can be represented as $\mathfrak{p}_{1} e_{i_{1}} \mathfrak{p}_{2} \cdots \mathfrak{p}_{r} e_{i_{r}} \mathfrak{p}_{r+1}$ and $\mathfrak{p}_{1}^{\prime} e_{j_{1}} \mathfrak{p}_{2}^{\prime} \cdots \mathfrak{p}_{r^{\prime}}^{\prime} e_{j_{r^{\prime}}} \mathfrak{p}_{r^{\prime}+1}^{\prime}$, correspondingly, where $\mathfrak{p}_{s}, \mathfrak{p}_{s^{\prime}}^{\prime}$ are paths in $T_{0}, 1 \leq s \leq r+1$, $1 \leq s^{\prime} \leq r^{\prime}+1$ and $e_{i_{t}}, e_{j_{t^{\prime}}} \in\left\{e_{1}, \ldots, e_{\mathfrak{m}}\right\}, 1 \leq t \leq r, 1 \leq t^{\prime} \leq r^{\prime}$.

Lemma 6.26 The map $\tau_{0}^{\prime}$

$$
h_{k}^{(0)} \mapsto \begin{cases}\varphi_{0}\left(h_{k}^{(0)}\right) & \text { for all } h_{k}^{(0)} \in \sigma, \sigma \notin \mathcal{P} \text { and } h_{k}^{(0)} \in T \cap \mathrm{Sh}, \\ \varphi_{0}\left(h^{(0)}(\mathfrak{c})\right) & \text { for all } \mathfrak{c} \in C^{(1)} \cup C^{(2)}, \\ x_{i}^{(0)} \varphi_{0}\left(h\left(e_{i}\right)\right) & \text { for all } h_{k}^{(0)}=h\left(e_{i}\right), i=1, \ldots, \mathfrak{m},\end{cases}
$$

induces a map $\tau_{0}$ from the set $\left\{h^{(0)}\right\}$ which extends to a homomorphism from $G_{\Omega_{0}}$ to $\mathfrak{T}_{0}$. 
Proof By [14, Lemma 6.14], the set

$$
\begin{aligned}
\left\{h_{k}^{(0)} \mid h_{k}^{(0)} \in \sigma, \sigma \notin \mathcal{P}\right\} \cup\left\{h_{k}^{(0)} \mid\right. & \left.h_{k}^{(0)} \in T \cap \mathrm{Sh}\right\} \\
& \cup h^{(0)}\left(C^{(1)}\right) \cup h^{(0)}\left(C^{(2)}\right) \cup\left\{h^{(0)}\left(e_{1}\right), \ldots, h^{(0)}\left(e_{\mathfrak{m}}\right)\right\}
\end{aligned}
$$

is a generating set of $G_{\Omega_{0}}$.

Notice that the map $\tau_{0}$ on the set

$$
S=\left\{h_{k}^{(0)} \mid h_{k}^{(0)} \in \sigma, \sigma \notin \mathcal{P}\right\} \cup\left\{h_{k}^{(0)} \mid h_{k}^{(0)} \in T \cap \operatorname{Sh}\right\} \cup h^{(0)}\left(C^{(1)}\right) \cup h^{(0)}\left(C^{(2)}\right)
$$

coincides with the composition of the epimorphism $\pi\left(v_{0}, v_{1}\right)$ and the homomorphism $\tau_{1}$, and so it extends to a homomorphism on the subgroup $\langle S\rangle$ of $G_{\Omega_{0}}$. In particular, the map $\tau_{0}$ coincides with $\varphi_{0}$ on the subgroup generated by all the items $h_{k}^{(0)} \notin \mathcal{P}$.

By [14, Lemma 6.14], since $\Omega_{0}$ is periodised, ie $\left[h^{(0)}\left(\mathfrak{c}_{1}\right), h^{(0)}\left(\mathfrak{c}_{2}\right)\right]=1$ for all cycles $\mathfrak{c}_{1}, \mathfrak{c}_{2} \in C^{(1)} \cup C^{(2)}$, it follows that all the words $h^{(0)}(\mu)^{-\varepsilon(\mu)} h^{(0)}(\Delta(\mu))^{\varepsilon(\Delta(\mu))}$ belong to the subgroup generated by $S$ and so

$$
\tau_{0}\left(h^{(0)}(\mu)^{-\varepsilon(\mu)} h^{(0)}(\Delta(\mu))^{\varepsilon(\Delta(\mu))}\right)=1 .
$$

We note that the words $\left[h^{(0)}\left(\mathfrak{c}_{1}\right), h^{(0)}\left(\mathfrak{c}_{2}\right)\right]$ are trivial elements in the subgroup generated by $S$ for all cycles $\mathfrak{c}_{1}, \mathfrak{c}_{2} \in C^{(1)} \cup C^{(2)}$. Therefore, in order to show that the map $\tau_{0}$ extends to a homomorphism from $G_{\Upsilon_{0}}$ to $\mathfrak{T}_{0}$ it suffices to show $\tau_{0}\left(u_{e i}^{h^{(0)}\left(e_{i}\right)} z_{e i}^{-1}\right)=1$ in $\mathfrak{T}_{0}, 1 \leq i \leq \mathfrak{m}$. Indeed,

$$
\tau_{0}\left(u_{e i}^{h^{(0)}\left(e_{i}\right)} z_{e i}^{-1}\right)=\varphi_{0}\left(u_{e i}\right)^{x_{i}^{(0)} \varphi_{0}\left(h^{(0)}\left(e_{i}\right)\right)} \varphi_{0}\left(z_{e i}^{-1}\right) .
$$

Since from the definition of $S_{0},\left[x_{i}^{(0)}, \varphi_{0}\left(u_{e i}\right)\right]=1$, it follows that

$$
\tau_{0}\left(u_{e i}^{h^{(0)}\left(e_{i}\right)} z_{e i}^{-1}\right)=\varphi_{0}\left(u_{e i}^{h^{(0)}\left(e_{i}\right)} z_{e i}^{-1}\right)=1 .
$$

To prove that $\tau_{0}$ extends to a homomorphism from $G_{\Omega_{0}}$ to $\mathfrak{T}_{0}$, it is left to show that $\tau_{0}\left(\left[h_{i}^{(0)}, h_{j}^{(0)}\right]\right)=1$, for all $h_{i}^{(0)}, h_{j}^{(0)}$ such that $\Re_{\Upsilon_{0}}\left(h_{i}^{(0)}, h_{j}^{(0)}\right)$. In fact, since the map extends to a homomorphism on the subgroup generated by $S$ we only need to check $\tau_{0}\left(\left[h_{i}^{(0)}, h_{j}^{(0)}\right]\right)=1$, for all $h_{j}^{(0)} \in \mathcal{P}$ so that $\Re \Upsilon_{0}\left(h_{i}^{(0)}, h_{j}^{(0)}\right)$. For every $P$-periodic solution $H^{(0)}, \operatorname{alph}\left(H_{i}^{(0)}\right) \subset \operatorname{alph}\left(H_{j}^{(0)}\right)$, for all $h_{i}^{(0)} \in \sigma, \sigma \in \mathcal{P}, h_{j}^{(0)} \in \mathcal{P}$. Recall that if $\Re \Upsilon_{0}\left(h_{i}^{(0)}, h_{j}^{(0)}\right)$, then $H_{i}^{(0)} \leftrightarrows H_{j}^{(0)}$ for all solutions $H^{(0)}$ of $\Omega_{0}$. Therefore, if $\Re \Upsilon_{0}\left(h_{i}^{(0)}, h_{j}^{(0)}\right.$ ) and $h_{j}^{(0)} \in \mathcal{P}$, then we have $h_{i}^{(0)} \in \sigma, \sigma \notin \mathcal{P}$.

Furthermore, since the periodic structure is not strongly singular and the set $\Re \Upsilon_{0}$ is completed, it follows that for all $h_{j}^{(0)} \in \mathcal{P}$ and for every $h_{i}^{(0)}$ such that $\Re \Upsilon_{0}\left(h_{i}^{(0)}, h_{j}^{(0)}\right)$, one has that $\Re_{\Upsilon_{0}}\left(h_{i}^{(0)}, h_{k}^{(0)}\right)$, for all $h_{j}^{(0)} \in \sigma, \sigma \in \mathcal{P}$. 
By properties of the elementary transformations, we have that $\Re_{\Upsilon_{1}}\left(h_{r}^{(1)}, h_{s}^{(1)}\right)$, for all the items $h_{r}^{(1)}$ which appear in the word $\pi\left(v_{0}, v_{1}\right)\left(h_{i}^{(0)}\right)$ and all items $h_{s}^{(1)}$ from the word $\pi\left(v_{0}, v_{1}\right)\left(h_{k}^{(0)}\right), h_{k}^{(0)} \in \sigma, \sigma \in \mathcal{P}$. By the induction hypothesis (IH2) on $\tau_{1}$, for any $y_{m}^{(1)}$ which appears in the word $\varphi_{0}\left(h_{i}^{(0)}\right)$, any $y_{n}^{(1)}$ from the word $\varphi_{0}\left(h_{k}^{(0)}\right)$ and any solution $H^{(1)}$ of $\Omega_{1}$ that factors through the branch, we have $\mathbb{A}\left(H^{(1)^{\prime}}\left(y_{m}^{(1)}\right)\right) \supset$ $\mathbb{A}\left(H_{i}^{(0)}\right)$ and $\mathbb{A}\left(H^{(1)^{\prime}}\left(y_{n}^{(1)}\right)\right) \supset \mathbb{A}\left(H_{k}^{(0)}\right)$, hence $H^{(1)^{\prime}}\left(y_{m}^{(1)}\right) \leftrightarrows H^{(1)^{\prime}}\left(y_{n}^{(1)}\right)$. By the induction hypothesis (IH1) on $\mathbb{H}_{1}$, we have that $\left(x_{n}^{(1)}, x_{m}^{(1)}\right) \in E_{d}\left(\Gamma_{1}\right)$ and so $\varphi_{0}\left(h_{i}^{(0)}\right) \leftrightarrows \varphi_{0}\left(h_{k}^{(0)}\right)$ in $\mathbb{H}_{1}$, for all $h_{k}^{(0)} \in \sigma, \sigma \in \mathcal{P}$ and, hence, in particular, $\varphi_{0}\left(h_{i}^{(0)}\right)$ belongs to the subgroup $\mathbb{K}$ of $\mathbb{H}_{1}$. Therefore, if $\Re \Upsilon_{0}\left(h_{i}^{(0)}, h_{j}^{(0)}\right)$ and $h_{j}^{(0)} \in \mathcal{P}$, then $\tau_{0}\left(\left[h_{i}^{(0)}, h_{j}^{(0)}\right]\right)=1$.

Lemma 6.27 The homomorphism $\tau_{0}: G_{\Omega_{0}} \rightarrow \mathfrak{T}_{0}$ makes diagram (11) commutative.

Proof The proof is analogous to the one of Lemma 6.15 .

Lemma 6.28 The subgroup $\mathbb{K}=\mathbb{A}\left(w\left(x^{(1)}\right)\right)$ of $\mathbb{H}_{1}$ is $E_{d}\left(\Gamma_{0}\right)$-coirreducible.

Proof The proof is analogous to the one of Lemma 6.16 .

Lemma 6.29 The group $\mathbb{H}_{0}$ satisfies the induction hypothesis (IH1): for every $x_{i}, x_{j} \in \mathbb{H}_{0}$, we have that $\left(x_{i}, x_{j}\right) \in E_{d}\left(\Gamma_{0}\right)$ if and only if $H^{\prime}\left(x_{i}\right) \leftrightarrows H^{\prime}\left(x_{j}\right)$ for all homomorphisms $H^{\prime}$ induced by solutions $H$ that factor through the branch. Furthermore, if $\left(x_{i}, x_{j}\right) \in E_{c}\left(\Gamma_{0}\right)$, then $H^{\prime}\left(x_{i}\right), H^{\prime}\left(x_{j}\right)$ belong to a cyclic subgroup for all homomorphisms $H^{\prime}$ induced by solutions $H$ that factor through the branch.

Proof The proof is analogous to the one of Lemma 6.18 .

Lemma 6.30 The homomorphism $\tau_{0}$ satisfies the induction hypothesis (IH2): for all $h_{i}^{(0)}$, if $\tau_{0}\left(h_{i}^{(0)}\right)=x_{i 1} \cdots x_{i k}$, then we have that $\mathbb{A}\left(H^{(0)^{\prime}}\left(x_{i j}\right)\right) \supset \mathbb{A}\left(H_{i}^{(0)}\right)$ and $\bigcap_{j=1}^{k} \mathbb{A}\left(H^{(0)^{\prime}}\left(x_{i j}\right)\right)=\mathbb{A}\left(H_{i}^{(0)}\right)$, for all solutions that factor through the branch.

Proof The proof is analogous to the one of Lemma 6.19.

We summarise the results of this section in the following.

Proposition 6.31 Let $\Omega_{0} \rightarrow \Omega_{1} \rightarrow \cdots \rightarrow \Omega_{q}$ be a branch of a tribal solution tree and assume that $\Omega_{1}$ satisfies the induction hypotheses (IH), (IH1) and (IH2); see page 784. If $\Omega_{0}=\langle\Upsilon, \Re \Upsilon\rangle$ is a generalised equation regular with respect to a periodic structure, then there exists a graph tower $\left(\mathfrak{T}_{0}, \mathbb{H}_{0}\right)$ and a homomorphism $\tau_{0}$ from $G_{\Omega_{0}}$ to $\mathfrak{T}_{0}$ such that for each solution that factors through the branch, there exists a homomorphism from $\mathfrak{T}_{0}$ to $\mathbb{G}$ that makes diagram (11) commutative. 


\subsection{Case 15}

Suppose that $\Omega_{0}$ is tribal of type 15 . Let $\Omega_{0} \rightarrow \Omega_{1} \rightarrow \cdots \rightarrow \Omega_{N}$ be a branch of the tribal tree $T_{0}\left(\Omega_{0}\right)$ such that all the epimorphisms $\pi\left(v_{i}, v_{i+1}\right): G_{R\left(\Omega_{i}\right)} \rightarrow G_{R\left(\Omega_{i+1}\right)}$ are isomorphisms, $i=0, \ldots, N-2$ and $\pi\left(v_{N-1}, v_{N}\right): G_{R\left(\Omega_{N-1}\right)} \rightarrow G_{R\left(\Omega_{N}\right)}$ is a proper epimorphism. It follows by construction of the tribal tree $T\left(\Omega_{0}\right)$, that one can subdivide the branch $\Omega_{0} \rightarrow \Omega_{1} \rightarrow \cdots \rightarrow \Omega_{N}$ as follows. Choose $0<n_{0}<n_{1}<\cdots<n_{k}=N$ so that:

- The quadratic part of $\Omega_{j}$ is nontrivial for all $j<n_{0}$ and $\Omega_{n_{0}}$ has trivial quadratic part, ie in the process the quadratic part of $\Omega_{0}$ is transferred to the nonquadratic part of $\Omega_{n_{0}}$ and the generalised equation $\Omega_{n_{0}}$ is the first one in the branch with trivial quadratic part.

- Every solution $H$ that factors through the branch is $P_{l}$-periodic in the section $\left[1, i_{l}\right]$ of the generalised equation $\Omega_{n_{l}}$ and $\Omega_{n_{l}}$ is periodised with respect to a regular periodic structure $\mathcal{P}\left(H, P_{l}\right)$. Furthermore, the section $\left[1, i_{l}\right]$ is transferred in the process from $\Omega_{n_{l}}$ to $\Omega_{n_{l+1}}, k=0, \ldots, N-1$.

We now use induction on $n_{k}$ to show there exists a graph tower $\mathfrak{T}_{0}$ and homomorphisms making diagram (11) commutative for all solutions that factor through the branch.

Suppose first that $k=1$. Then the existence of the graph tower $\mathfrak{T}_{0}$ for $G_{\Omega_{0}}$ and the required homomorphisms follows by Proposition 6.12. Assume now that $k>1$. By construction, declaring the section $\left[1, i_{k-1}\right]$ to be the active section of $\Omega_{n_{k-1}}$, the generalised equation $\Omega_{n_{k-1}}$ is periodised with respect to a regular periodic structure $\mathcal{P}\left(H, P_{k-1}\right)$. Hence, by Proposition 6.31, there exists a graph tower $\mathfrak{T}_{k-1}$ for $\Omega_{n_{k-1}}$ that satisfies the required properties. This proves the base of induction.

Assume by induction that there exists a graph tower $\mathfrak{T}_{2}$ for $G_{\Omega_{n_{2}}}$. Then, again using Proposition 6.31, we conclude that there exists a graph tower $\mathfrak{T}_{1}$ and the required homomorphisms for $G_{\Omega_{n_{1}}}$.

Finally, since there exists a tower $\mathfrak{T}_{1}$ for $G_{\Omega_{n_{1}}}$, by Proposition 6.12, there exists a graph tower $\mathfrak{T}_{0}$ satisfying the required properties for the group $G_{\Omega_{n_{0}}}$.

We arrive at the following:

Proposition 6.32 Let $\Omega_{0} \rightarrow \Omega_{1} \rightarrow \cdots \rightarrow \Omega_{q}$ be a branch of a tribal solution tree and assume that $\Omega_{1}$ satisfies the induction hypotheses (IH), (IH1) and (IH2); see 784. If $\Omega_{0}=\langle\Upsilon, \Re \Upsilon\rangle$ is a tribal generalised equation of type 15 , then there exists a graph tower $\left(\mathfrak{T}_{0}, \mathbb{H}_{0}\right)$ and a homomorphism $\tau_{0}$ from $G_{\Omega_{0}}$ to $\mathfrak{T}_{0}$ such that for each solution that factors through the branch, there exists a homomorphism from $\mathfrak{T}_{0}$ to $\mathbb{G}$ that makes diagram (11) commutative. 


\subsection{Linear case}

Suppose that $\Omega_{0}$ is tribal of linear type. In this case, the generalised equation $\Omega_{1}$ is simply the kernel of $\Omega_{0}, \operatorname{Ker}\left(\Omega_{0}\right)=\Omega_{1}$.

By induction, let $\mathfrak{T}_{1}$ and $\tau_{1}$ be the corresponding tower and homomorphism for $\Omega_{1}$. Then, by Corollary 4.9,

$$
\mathbb{G}_{R\left(\Omega_{0}\right)} \simeq \mathbb{G}\left[h_{1}^{(1)}, \ldots, h_{\rho_{\Omega_{1}}}^{(1)}, z_{1}, \ldots, z_{l}\right] / R\left(\bar{\Omega}_{1} \cup \mathcal{K}\right),
$$

Let $i$ be the corresponding isomorphism.

Define the graph $\Gamma_{0}$ by

- $V\left(\Gamma_{0}\right)=V\left(\Gamma_{1}\right) \cup\left\{x_{1}^{(0)}, \ldots, x_{l}^{(0)}\right\}$;

- $E_{c}\left(\Gamma_{0}\right)=E_{c}\left(\Gamma_{1}\right)$;

- $E_{d}\left(\Gamma_{0}\right)=E_{d}\left(\Gamma_{1}\right) \cup \mathcal{K}_{1} \cup \mathcal{K}_{2}$, where $\mathcal{K}_{1}=\left\{\left(x_{i}^{(0)}, x_{j}^{(0)}\right) \mid\left[z_{i}, z_{j}\right] \in \mathcal{K}\right\}$ and $\mathcal{K}_{2}$ is the set of all pairs $\left(x_{i}^{(0)}, x_{k}^{(1)}\right)$, so that $x_{k}^{(1)}$ appears in the image of a free variable $z_{m}$ under the word map $\tau_{1} \pi\left(v_{0}, v_{1}\right)$ and $\left[z_{i}, z_{m}\right] \in \mathcal{K}$.

The group $\mathbb{H}_{0}$ is defined to be the partially commutative group $\mathbb{H}\left(\Gamma_{0}\right)$. We now define the tower $\mathfrak{T}_{0}$ as follows: $\mathfrak{T}_{0}=\left\langle\mathfrak{T}_{1}, x_{1}^{(0)}, \ldots, x_{l}^{(0)} \mid \mathcal{K}_{1}, \mathcal{K}_{2}\right\rangle$ (recall that, by definition, there is a canonical epimorphism $\pi$ from $\mathbb{H}_{1}$ to $\mathfrak{T}_{1}$, which defines a natural correspondence between the generators of $\mathfrak{T}_{1}$ and $\left.\mathbb{H}_{1}\right)$.

We now show that the triple $\mathfrak{T}_{0}, \mathbb{H}_{0}$ and $i \tau_{0}$ is a graph tower associated to $G_{\Omega}$. Since $i$ is an isomorphism, it implies that diagram (11) is commutative. We are left to show that $i \tau_{0}$ satisfies the induction hypotheses (IH1) and (IH2). Since $\mathbb{H}_{1}$ satisfies the condition (IH1) for the homomorphisms induced by solutions of $\Omega_{0}$ and since every solution of $\Omega_{1}$ induces a solution of $\Omega_{0}$, it follows that $\mathbb{H}_{0}$ satisfies the assumption (IH1) for the family of solutions of $\Omega_{1}$. Let $z_{m}=h_{m}$ be any item of $\Omega_{0}$ which has been eliminated. It suffices to prove that for all $x_{k}^{(1)}$ in $i \tau_{1} \pi\left(v_{0}, v_{1}\right)$ and any solution that factors through the branch we have that

$$
\mathbb{A}\left(H^{(1)}\left(x_{k}^{(1)}\right)\right) \supset \mathbb{A}\left(H_{m}^{(0)}\right) .
$$

The latter follows since $h_{m}=w_{v}, h_{m}$ belongs to the tribe $t\left(\lambda_{i}\right)$, any $h_{k}$ in $w_{v}$ dominates the tribe $t\left(\lambda_{i}\right)$ (ie for any solution that factors through the branch $\mathbb{A}\left(H^{(1)}\left(x_{k}^{(1)}\right)\right) \supset$ $\left.\mathbb{A}\left(H_{m}^{(0)}\right)\right)$ and $\tau_{1}$ satisfies the induction hypothesis (IH2) for the items $z_{i}, i=1, \ldots, l$ in $\Omega_{0}$.

Finally, we summarise the results of this whole section in the theorem below. 
Theorem 6.33 Let $\Omega_{0} \rightarrow \cdots \rightarrow \Omega_{q}$ be a branch of the tribal solution tree $T_{\text {sol }}(\Omega)$. Then there exists a homomorphism from $G_{\Omega_{0}}$ to a graph tower $\mathfrak{T}_{0}$ which makes diagram (11) commutative. Furthermore, the graph tower $\mathfrak{T}_{0}$ and the corresponding homomorphism can be constructed effectively.

\section{Graph towers are fully residually partially commutative}

In Section 6, we proved that given a branch $\Omega_{0} \rightarrow \cdots \rightarrow \Omega_{q}$ of the tribal solution tree, one can effectively construct a graph tower $\mathfrak{T}_{0}$ and a homomorphism $\tau_{0}: G_{\Omega_{0}} \rightarrow \mathfrak{T}_{0}$ that makes diagram (11) commutative. In this section, we show that the graph tower $\mathfrak{T}_{0}$ is discriminated by $\mathbb{G}$ by the family of homomorphisms that factor through the branch.

Theorem 7.1 Let $(\mathfrak{T}, \mathbb{H})$ be the graph tower associated to a branch of a tribal solution tree. Then the graph tower $\mathfrak{T}$ is discriminated by $\mathbb{G}$ by the family of homomorphisms induced by solutions that factor through the branch.

Proof We prove the statement by induction on the height of the graph tower. Suppose that the height of the graph tower is 0 . Then $\mathfrak{T}$ is the partially commutative group associated to a leaf of the tree $T_{\text {sol }}(\Omega)$ for some generalised equation $\Omega$ and so, by [14, Proposition 9.1], the graph tower $\mathfrak{T}$ is discriminated by $\mathbb{G}$ by the family of solutions of the generalised equation.

Suppose that if $\mathfrak{T}$ is a graph tower of height less than or equal to $l-1$, then $\mathfrak{T}$ is discriminated by $\mathbb{G}$ by the family of homomorphisms induced by solutions that factor through the branch.

Let $\mathfrak{T}_{0}$ be a graph tower of height $l$. By Lemma 5.3, the graph tower $\mathfrak{T}_{0}$ has a decomposition as an amalgamated product (or HNN-extension). We now analyse each of the possible decompositions.

Notice that if $\mathfrak{T}_{0}$ is of type (a1), then it only appears in the construction of the leaves of $T_{\text {sol }}$, ie the graph tower $\mathfrak{T}_{1}$ coincides with the associated partially commutative group $\mathbb{H}\left(\Gamma_{1}\right)$. In this case, since $\mathbb{K}^{\perp}$ is directly indecomposable, it follows from [14, Corollary 2.11] that the family of solutions $H$ that factor through the branch and which map the variables $x^{(0)}$ into irreducible elements of the subgroup $\mathbb{K}^{\perp}$ is a discriminating family.

Assume now that the graph tower $\mathfrak{T}_{0}$ is of type (a2). This case appears either in the construction of a leaf, in the presence of a periodic structure or in the particular case when the quadratic equation in the normal form does not satisfy the properties $\circledast$ and $\circledast \circledast$; see Definition 5.2 (and $\mathbb{K}^{\perp}$ is $E_{c}(\Gamma)$-abelian). 
If it appears in the construction of a leaf, the result follows analogously to case (a1).

Let us now consider the remaining cases. We begin with an observation. Let $\mathbb{G}_{\mathbb{K}}$ be the canonical parabolic subgroup such that for solutions $H$ that factor through the branch we have that $\left\langle\operatorname{alph}\left(H^{\prime}(\mathbb{K})\right)\right\rangle=\mathbb{G}_{\mathbb{K}}$ (recall that the group $\mathbb{G}_{\mathbb{K}}$ is well defined since items of generalised equations have associated parabolic subgroups). Since $\mathbb{G}_{\mathbb{K}}^{\perp} \leftrightarrows \mathbb{G}_{\mathbb{K}}$, by the induction hypothesis (IH1), it follows that $\mathbb{G}_{\mathbb{K}}^{\perp}<\mathbb{K}^{\perp}$. Since $\mathbb{K}^{\perp}$ is $E_{c}\left(\Gamma_{1}\right)$-abelian, so is $\mathbb{G}_{\mathbb{K}}^{\perp}$. Since for the discriminating family of solutions the subgroup which is defined by $H^{\prime}\left(\mathbb{K}^{\perp}\right)$ is cyclic and $\left\langle\operatorname{alph}\left(H^{\prime}\left(\mathbb{G}_{\mathbb{K}}^{\perp}\right)\right)\right\rangle=\mathbb{G}_{\mathbb{K}}^{\perp}$, it follows that $\mathbb{G}_{\mathbb{K}}^{\perp}$ is a cyclic canonical parabolic subgroup generated by the generator $a$. Let $C_{\mathbb{G}}(a)=\langle a\rangle \times \mathbb{A}(a)$. Since $\langle a\rangle=\mathbb{G}_{\mathbb{K}} \perp \leftrightarrows \mathbb{G}_{\mathbb{K}}$, we have that $\mathbb{G}_{\mathbb{K}}<\mathbb{A}(a)$. On the other hand, since $\mathbb{A}(a) \leftrightarrows H^{\prime}\left(\mathbb{K}^{\perp}\right)$, by the induction hypothesis (IH1), it follows that $\mathbb{A}(a)<\mathbb{K}$ and so $\mathbb{A}(a)<\mathbb{G}_{\mathbb{K}}$. Hence $\mathbb{G}_{\mathbb{K}}=\mathbb{A}(a)$ and $\mathbb{G}_{\mathbb{K}} \perp=\langle a\rangle$. Furthermore, since $C_{\mathbb{G}}(a)<H^{\prime}\left(C_{\mathfrak{T}_{1}}\left(\mathbb{K}^{\perp}\right)\right)<C_{\mathbb{G}}\left(H^{\prime}\left(\mathbb{K}^{\perp}\right)\right)<C_{\mathbb{G}}(a)$ for all homomorphisms $H^{\prime}$ induced by the discriminating family, we conclude that $C_{\mathbb{G}}(a)=\mathbb{G}_{C_{\mathfrak{T}_{1}}}\left(\mathbb{K}^{\perp}\right)$.

Since $\mathfrak{T}_{0}$ is an amalgamated product, it follows that every nontrivial element $e$ of $\mathfrak{T}_{0}$ can be written in the reduced form as $a_{1} b_{1} a_{2} b_{2} \cdots a_{k} b_{k} a_{k+1}$, where $a_{2}, \ldots, a_{k+1} \notin$ $C_{\mathfrak{T}_{1}}\left(\mathbb{K}^{\perp}\right)$ and $b_{1}, \ldots, b_{k}$ are words in the generators $\left\{x_{1}^{l}, \ldots, x_{m_{l}}^{l}\right\}$. Hence, there exist $w_{i} \in \mathbb{K}^{\perp}$ such that $\left[a_{i}, w_{i}\right] \neq 1, i=2, \ldots, k+1$.

Since, by induction hypothesis, $\mathfrak{T}_{1}$ is discriminated by $\mathbb{G}$ by the family of homomorphisms induced by the set of solutions, there exists a homomorphism $H^{(1)^{\prime}}$ which is injective on the finite set $\left\{\left[a_{i}, w_{i}\right] \mid i=2, \ldots, k+1\right\}$. Therefore

$$
H^{(1)^{\prime}}\left(\left[a_{i}, w_{i}\right]\right)=\left[H^{(1)^{\prime}}\left(a_{i}\right), a^{l_{i}}\right] \neq 1 .
$$

Hence $H^{(1)^{\prime}}\left(a_{i}\right) \notin C_{\mathbb{G}}(a)$ and, in particular, using the above observation, $H^{(1)^{\prime}}\left(a_{i}\right) \notin$ $\mathbb{G}_{C_{\mathfrak{T}_{1}}\left(\mathbb{K}^{\perp}\right)}$.

The homomorphism $H^{(1)^{\prime}}$ induces a homomorphism $H^{(1)^{\prime \prime}}$ from $\mathfrak{T}_{0}$ to the group

$$
A=\mathbb{G} *_{\mathbb{G}_{C_{\mathfrak{T}_{1}}}(\mathbb{K} \perp)}\left(\mathbb{G}_{C_{\mathfrak{T}_{1}}\left(\mathbb{K}^{\perp}\right)} \times\left\langle x_{1}^{l}, \ldots, x_{m_{l}}^{l} \mid\left[x_{i}^{l}, x_{j}^{l}\right], 1 \leq i<j \leq n\right\rangle\right),
$$

ie $H^{(1)^{\prime \prime}}$ is a homomorphism from $\mathfrak{T}_{0}$ to the extension of the centraliser of the element $a$ :

$$
A=\mathbb{G} * C_{\mathbb{G}}(a)\left\langle C_{\mathbb{G}}(a), x_{1}^{l}, \ldots, x_{m_{l}}^{l} \mid\left[C_{\mathbb{G}}(a), x_{i}^{l}\right]=1,\left[x_{i}^{l}, x_{j}^{l}\right]=1\right\rangle .
$$

Moreover, the image $H^{\prime}\left(a_{1}\right) b_{1} \cdots b_{k} H^{\prime}\left(a_{k+1}\right)$ of $e$ in $\mathbb{G}$, is a reduced element of the

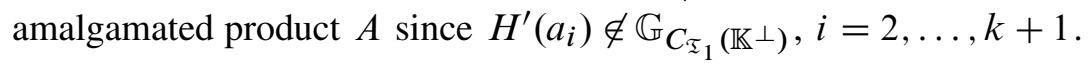

Furthermore, in all the cases under consideration (for periodic structures and for exceptional subcases of the quadratic case), we have that any solution $H^{(0)}$ that factors 
through the branch is the composition of a canonical automorphism $\phi$ of $G_{R\left(\Omega_{0}\right)}$, the epimorphism $\pi\left(v_{0}, v_{1}\right)$ and a solution $H^{(1)}$. Moreover, notice that any such automorphism $\phi$ induces an automorphism of $A$ that fixes $\mathbb{G}$. Hence the family of solutions $H^{(0)}$ that factor through the branch defines a family of homomorphisms from $A$ to $\mathbb{G}$ that are obtained from a (nontrivial) solution (induced by $\pi\left(v_{0}, v_{1}\right) H^{(1)}$ ) by precomposing with canonical automorphisms.

By [13, Lemma 4.17; 14, Corollary 2.11], the group $A$ (an extension of a centraliser of the canonical generator $a$ ) is discriminated by $\mathbb{G}$ by this family of homomorphisms. Therefore, we conclude that $\mathfrak{T}_{0}$ is discriminated by $\mathbb{G}$ by the family of homomorphisms induced by the solutions that factor through the branch.

Assume that $\mathfrak{T}_{0}$ is of type (b1) or (b2). These cases occur in the presence of a periodic structure or in the particular case when the quadratic equation in the normal form does not satisfy the properties $\circledast$ and $\circledast \circledast$ from Definition 5.2. By Remarks 6.17 and 6.4, the homomorphisms $H^{\prime}$ induced by solutions that factor through the branch (only in the case (b1)) satisfy that $H^{\prime}(u)$ and $H^{\prime}\left(x_{i}^{l}\right)$ are irreducible elements of $\mathbb{G}_{\mathbb{K}} \perp$. Now the proof is analogous to the case (a2).

Suppose that $\mathfrak{T}_{0}$ is of type c). This case only occurs when the quadratic equation satisfies properties $\circledast$ and $\circledast \circledast$ from Definition 5.2.

Claim The subgroup of $\mathfrak{T}_{1}$ which is generated by $C_{\mathfrak{T}_{1}}\left(\mathbb{K}^{\perp}\right)$ and $\varphi_{0}\left(\lambda_{i}\right)$, where $\lambda_{i}$ is a quadratic-coefficient base, $i=2 g+1, \ldots, m$ is the direct product

$$
\left\langle C_{\mathfrak{T}_{1}}\left(\mathbb{K}^{\perp}\right), \varphi_{0}\left(\lambda_{2 g+1}\right), \ldots, \varphi_{0}\left(\lambda_{m}\right)\right\rangle=C_{\mathfrak{T}_{1}}\left(\mathbb{K}^{\perp}\right) \times\left\langle\varphi_{0}\left(\lambda_{2 g+1}\right), \ldots, \varphi_{0}\left(\lambda_{m}\right)\right\rangle .
$$

Let us prove the claim. In fact, we show that the subgroup $C$ generated by $\mathbb{K}^{\perp}$ and $C_{\mathfrak{T}_{1}}\left(\mathbb{K}^{\perp}\right)$ is a direct product. Then, since $\varphi_{0}\left(\lambda_{i}\right) \in \mathbb{K}^{\perp}$, the claim will follow. By Remark 6.4, for the family of homomorphisms $H^{\prime}$ induced by the set of solutions that factor through the branch, we have that for all minimal items $h_{i}$, the image $H_{i}^{\prime}$ is a block element of $\mathbb{G}_{\mathbb{K}^{\perp}}$ and $\mathbb{A}\left(H_{i}^{\prime}\right)=\mathbb{A}\left(\mathbb{G}_{\mathbb{K}^{\perp}}\right)$. In particular, it follows that $\mathbb{G}_{\mathbb{K}^{\perp}}$ is a (nonabelian) directly indecomposable canonical parabolic subgroup. By the description of centralisers in partially commutative groups, it follows that $\mathbb{G}_{\mathbb{K}} \perp \cap C_{\mathbb{G}}\left(\mathbb{G}_{\mathbb{K}} \perp\right)=1$ and so $\mathbb{G}_{\mathbb{K}} \perp \leftrightarrows \mathbb{G}_{C_{\mathfrak{T}_{1}}\left(\mathbb{K}^{\perp}\right)}$. By induction hypothesis, we have that the subgroup $C$ is discriminated by the subgroup $\mathbb{G}_{\mathbb{K}^{\perp}} \times \mathbb{G}_{C_{\mathfrak{T}_{1}}\left(\mathbb{K}^{\perp}\right)}$ and so $C$ is the direct product of $\mathbb{K}^{\perp}$ and $C_{\mathfrak{T}_{1}}\left(\mathbb{K}^{\perp}\right)$. Indeed since $C=\mathbb{K}^{\perp} \cdot C_{\mathfrak{T}_{1}}\left(\mathbb{K}^{\perp}\right)$ and $\left[\mathbb{K}^{\perp}, C_{\mathfrak{T}_{1}}\left(\mathbb{K}^{\perp}\right)\right]=1$, we are left to show that $\mathbb{K}^{\perp} \cap C_{\mathfrak{T}_{1}}\left(\mathbb{K}^{\perp}\right)=1$. Suppose that there exist $u \in \mathbb{K}^{\perp}$ and $v \in C_{\mathfrak{T}_{1}}\left(\mathbb{K}^{\perp}\right)$ so that $u=v$ in $C$. Then $H^{\prime}(u) \in \mathbb{G}_{\mathbb{K}^{\perp}}, H^{\prime}(v) \in \mathbb{G}_{C_{\mathfrak{T}_{1}}\left(\mathbb{K}^{\perp}\right)}$ and $H^{\prime}(u)=H^{\prime}(v)$. Since $\mathbb{G}_{\mathbb{K}} \perp \leftrightarrows \mathbb{G}_{C_{\mathfrak{T}_{1}}\left(\mathbb{K}^{\perp}\right)}$, so $H^{\prime}(u)=H^{\prime}(v)=1$ for all homomorphisms $H^{\prime}$. It follows that $u=v=1$. 
Our strategy to prove that $\mathfrak{T}_{0}$ is discriminated by $\mathbb{G}$ is as follows. As above, to prove that $\mathfrak{T}_{0}$ is discriminated, it suffices to prove that so is

$$
M=\mathbb{G} *_{H^{\prime}\left(C_{\mathfrak{T}_{1}}\left(\mathbb{K}^{\perp}\right)\right) \times E}\left(\left\langle E, x_{1}^{l}, \ldots, x_{n}^{l} \mid W\right\rangle \times H^{\prime}\left(C_{\mathfrak{T}_{1}}\left(\mathbb{K}^{\perp}\right)\right)\right),
$$

where $E=H^{\prime}\left(\left\langle\varphi_{0}\left(\lambda_{2 g+1}\right), \ldots, \varphi_{0}\left(\lambda_{m}\right)\right\rangle\right)$. To prove the latter, we follow the standard argument for limit groups over free groups.

In short, a key statement to prove that a retracting surface into a limit group $L$ is discriminated is the existence of a simple closed curve (scc) which is mapped nontrivially by the retraction; see [52, Lemma 5.13]. This scc induces a decomposition of the surface as an amalgamated product or an HNN-extension. The homomorphisms obtained from the retraction by precomposing it with Dehn twists along this curve map the curve to big powers of the image of the curve under the retraction. Since limit groups have the BP property (see Wilton [58, Lemma 4.13]) and are CSA, a standard argument shows that the group is discriminated by $L$; see [52, Theorem 5.12]. In our case, we prove in Lemma 6.5 that there exists a nontrivial curve $U$ which is mapped not only nontrivially, but to irreducible elements for all solutions that factor through the branch. Furthermore, we prove in [14, Lemma 4.17] that partially commutative groups have the BP property with respect to irreducible elements and so $M$ is discriminated by $\mathbb{G}$.

A detailed proof in the free group case can be found in [58, Proposition 4.22]. We now explain which statements need to be replaced in [58, Proposition 4.22] and how in our context, the rest of the proof is verbatim. As we mentioned above, there are two adjustments required for the argument in [58] to go through: firstly, free groups satisfy the BP property (see [58, Lemma 4.13]) and secondly, limit groups over free groups are CSA. In general, partially commutative groups do not have the BP property (see Blatherwick [7]). In our case, since, by induction, we assume that $\mathfrak{T}_{1}$ is discriminated by the family of homomorphisms that factor through the branch, it follows that the images of $v_{i}, w_{j} \in \mathfrak{T}_{1}$ under the discriminating family are block elements (see Remark 6.4). Furthermore, by Lemma 6.5, there exists a nontrivial curve $U$ which is mapped to irreducible elements for all solutions that factor through the branch. We prove in [14, Lemma 4.17] that partially commutative groups have the BP property with respect to irreducible elements. Therefore, the argument given in [58, Proposition 4.22] goes through, using [14, Lemma 4.17] instead of [58, Lemma 4.13].

The argument of [58] uses the CSA property in [58, Example 4.21]. In general partially commutative group do not have the CSA property, but we can apply the argument from [58, Example 4.21] in our context as follows. Let $\Sigma, \zeta, S, S^{\prime}$ and $t$ be as in [58, Example 4.21]. By assumption, we have that $H^{\prime}(S)$ is nonabelian and we want to show that $H^{\prime}\left(S^{\prime}\right)$ is also nonabelian. 
Towards a contradiction, assume that $H^{\prime}\left(S^{\prime}\right)$ is abelian. By induction hypothesis, the discriminating family of homomorphisms $H^{\prime}$ induced by the solutions has the property that if $h_{i}$ is a minimal item, then $H_{i}^{\prime}$ is a block element and there exists an irreducible element $H(U) \in H^{\prime}\left(S^{\prime}\right)$. It follows that if the image $H^{\prime}\left(S^{\prime}\right)$ is abelian, then it is cyclic. In partially commutative groups, cyclic subgroups satisfy the property that if $H^{\prime}\left(S^{\prime}\right) \cap H^{\prime}\left(S^{\prime}\right)^{H^{\prime}(t)}$ is nontrivial, then $H^{\prime}(t)$ commutes with $H^{\prime}\left(S^{\prime}\right)$ and hence $H^{\prime}(S)$ is abelian, deriving a contradiction.

Now the argument is identical to the one given in [58, Proposition 4.22].

\section{Irreducible components}

If a group $G$ is equationally Noetherian, then every algebraic set $V$ in $G^{n}$ is a finite union of its irreducible components. Using the duality between the categories of algebraic sets and coordinate groups, one can conclude that if $G$ is equationally Noetherian, then any coordinate group is a subdirect product of coordinate groups of the (finitely many) irreducible components, [3].

Since partially commutative groups are equationally Noetherian, any finitely generated residually $\mathbb{G}$ group is a subdirect product of the direct product of finitely many limit groups over $\mathbb{G}$.

In the case of free groups, Kharlampovich and Miasnikov, [38], used the MakaninRazborov process to describe, on the one hand, an embedding of a finitely generated fully residually free group into a graph tower and, on the other one, an embedding of a finitely generated residually free group into the direct product of limit groups. In a recent work on the structure of finitely presented residually free groups, Bridson, Howie, Miller and Short [10] gave a different (canonical) construction of such embeddings.

The aim of this section is to generalise the aforementioned results and show that given a limit group $G$ over $\mathbb{G}$, ie a finitely generated fully residually $\mathbb{G}$ group, one can effectively construct an embedding of $G$ into a graph tower; and given a finitely generated residually $\mathbb{G}$ group $G$, one can effectively construct an embedding of $G$ into the direct product of limit groups over $\mathbb{G}$.

\section{Limit groups over $\mathbb{G}$ are subgroups of graphs towers}

Theorem 8.1 Let $G$ be a limit group over a partially commutative group $\mathbb{G}$. Then $G$ is a subgroup of a graph tower $(\mathfrak{T}, \mathbb{H})$. Furthermore if $G$ is given by its finite radical presentation, then the graph tower $(\mathfrak{T}, \mathbb{H})$ and the embedding can be effectively constructed. 
Proof Let $G$ be a limit group over $\mathbb{G}$ and $T_{\text {sol }}$ be the tribal solution tree associated to $G$. Since the tribal solution tree $T_{\text {sol }}$ is finite, it follows by Lemma 2.6, that there exists a branch of the solution tree $T_{\text {sol }}$ so that the subfamily of homomorphisms that factors through this branch is a discriminating family of $G$ into $\mathbb{G}$. We call this branch a fundamental branch of $G$ and any discriminating family of homomorphisms that factors through the fundamental branch is called a fundamental sequence.

Let $\mathfrak{T}$ be the graph tower associated to the fundamental branch. Since by Theorem 7.1 the graph tower $\mathfrak{T}$ is discriminated by $\mathbb{G}$ by the family of homomorphisms induced by a fundamental sequence of solutions associated to $G$ and since diagram (11) is commutative, it follows that $\operatorname{ker}\left(G_{\Omega} \rightarrow G_{R(\Omega)}\right)<\operatorname{ker} \tau$. Hence, by the universal property of the quotient, $\tau$ induces a homomorphism $\tau^{\prime}$ from $G_{R(\Omega)}$ to $\mathfrak{T}$. Since the fundamental sequence that discriminates $G$ into $\mathbb{G}$ factors through $\mathfrak{T}$, it follows that the composition of $\pi: G \rightarrow G_{R(\Omega)}$ and $\tau$ is an embedding of $G$ into $\mathfrak{T}$.

We now record some properties of limit groups over partially commutative groups.

\section{Proposition 8.2 (1) Every limit group over $\mathbb{G}$ is torsion free.}

(2) Every 2-generated limit group is either free or free abelian.

(3) Every solvable subgroup of a limit group is abelian.

(4) Let $\mathcal{G}$ be an algebraic group over $\mathbb{R}$ in which $\mathbb{G}$ embeds. Then for any limit group $G$ over $\mathbb{G}$ there exists an embedding $G \hookrightarrow \mathcal{G}$. In particular, $G$ embeds into $\mathrm{SL}_{n}(\mathbb{R})$. The natural map $G \rightarrow \mathrm{PSL}_{n}(\mathbb{R})$ is also an embedding.

(5) Let $\mathbb{G}$ be a partially commutative group and let $(\mathfrak{T}, \mathbb{H})$ be a graph tower associated to a limit group $G$ over $\mathbb{G}$. Then abelian subgroups of $\mathfrak{T}$ (and thus of $G$ ) are free, and there is a uniform bound on their rank.

Proof All but the last statement follow from definition using general arguments; see for example [58]. To prove the last one we use the fact that every limit group over $\mathbb{G}$ is a subgroup of a graph tower.

First notice that since, by Theorem 7.1 , the group $\mathfrak{T}$ is discriminated by $\mathbb{G}$, then any abelian subgroup of $\mathfrak{T}$ is torsion free. Let $A$ be an abelian subgroup of $\mathfrak{T}$. Let us prove that the rank of $A$ is uniformly bounded by induction on the height of $\mathfrak{T}$. If the height is 0 , then the graph tower coincides with the partially commutative group $\mathbb{G}$ and hence the rank of an abelian subgroup is bounded by the number of vertices in a maximal clique of the graph $\Gamma$ or, more coarsely, it is bounded by the number of generators of $\mathbb{G}$. 
Let $\mathfrak{T}=\mathfrak{T}^{l}$ be of height $l$. By Lemma 5.3, the group $\mathfrak{T}$ admits a decomposition as an amalgamated product. Let $T$ be the Bass-Serre tree associated to the decomposition of $\mathfrak{T}$. Notice that the rank of an abelian subgroup of $\mathfrak{T}^{l-1}$ is uniformly bounded by $N_{l-1}$ by induction hypothesis. Furthermore, the other vertex group is a direct product of a subgroup of $\mathfrak{T}^{l-1}$ and either a free, or a free abelian or a surface group. Hence in all the cases, we conclude that the rank of an abelian subgroup of this vertex group is uniformly bounded by $N_{l-1}+m_{l}$. If the subgroup $A$ fixes a vertex of $T$ then $A$ is a subgroup of a (conjugate) of a vertex group and so its rank is uniformly bounded by $N_{l-1}+m_{l}$. Otherwise, $A$ fixes a line $T_{A}$ in $T$, on which it acts by translations. The quotient $\Delta=A \backslash T_{A}$ is topologically a circle, after some collapses $\Delta$ is an HNN-extension, so the rank of $A$ is uniformly bounded by $N_{l-1}+m_{l}+1$.

\section{Residually partially commutative groups}

Our goal now is to prove that given a finitely generated residually $\mathbb{G}$ group $G$, one can effectively construct an embedding of $G$ into the direct product of limit groups over $\mathbb{G}$.

Theorem 8.3 Let $\mathbb{G}$ be a partially commutative group and let $G$ be a finitely generated residually $\mathbb{G}$ group. Then one can effectively construct finitely many fully residually $\mathbb{G}$ graph towers $\mathfrak{T}_{1}, \ldots, \mathfrak{T}_{k}$ and homomorphisms $p_{i}$ from $G$ to $\mathfrak{T}_{i}, i=1, \ldots, k$, so that any homomorphism from $G$ to $\mathbb{G}$ factors through a graph tower $\mathfrak{T}_{i}$, for some $i=1, \ldots, k$, ie for any homomorphism $\varphi: G \rightarrow \mathbb{G}$ there exist $i \in\{1, \ldots, k\}$ and a homomorphism $\varphi_{i}: \mathfrak{T}_{i} \rightarrow \mathbb{G}$ so that $\varphi=p_{i} \varphi_{i}$. In particular, $G$ is a subgroup of the direct product of the graph towers $\mathfrak{T}_{i}, i=1, \ldots, k$ and a subdirect product of the direct product of groups $p_{i}(G)<\mathfrak{T}_{i}, i=1, \ldots, k$.

Notice that $p_{i}(G)<\mathfrak{T}_{i}, i=1, \ldots, k$, are finitely generated subgroups of the graph towers $\mathfrak{T}_{i}$. Since the graph towers $\mathfrak{T}_{i}$ are discriminated by $\mathbb{G}$, it follows that $p_{i}(G)$, $i=1, \ldots, k$ are limit groups over $\mathbb{G}$.

Since partially commutative groups are equationally Noetherian, any finitely generated residually $\mathbb{G}$ group admits a finite radical presentation.

Corollary 8.4 Let $G$ be a finitely generated residually $\mathbb{G}$ group given by its finite radical presentation. Then one can effectively construct an embedding of $G$ into the direct product of finitely many limit groups over $\mathbb{G}$.

It is worthwhile mentioning that, in general, the decomposition we construct is not minimal, ie $G$ might be presented as the subdirect product of a direct product of (strictly) less than $k$ limit groups. 
Proof of Theorem 8.3 Let $G$ be a finitely generated residually $\mathbb{G}$ group and let $T_{\text {sol }}(G)$ be the tribal solution tree associated to $G$. For each branch $B: \Omega_{0, B} \rightarrow$ $\Omega_{1, B} \rightarrow \cdots \rightarrow \Omega_{n, B}$ of the tree $T_{\text {sol }}(G)$, as in Section 6 , we construct a graph tower $\mathfrak{T}_{B}$ and a homomorphism $\tau_{B}: G_{\Omega_{0, B}} \rightarrow \mathfrak{T}_{B}$ that makes diagram (11) commutative. By Theorem 7.1, each graph tower associated to a branch of the tree $T_{\text {sol }}(G)$ is discriminated by $\mathbb{G}$.

Let $\pi_{B}$ be the homomorphism from $G$ to $G_{\Omega_{0, B}}$ constructed in [14, Section 3.3] and set $p_{B}: G \rightarrow \mathfrak{T}_{B}$ to be $\pi_{B} \tau_{B}$. Since the tree $T_{\text {sol }}(G)$ describes all the homomorphisms from $G$ to $\mathbb{G}$, by the commutativity of diagram (11), we conclude that every homomorphism from $G$ to $\mathbb{G}$ factors through some graph tower $\mathfrak{T}_{B}$.

Furthermore, since the group $G$ is residually $\mathbb{G}$, it follows that the homomorphisms $p_{B}: G \rightarrow \mathfrak{T}_{B}$ induce an embedding of $G$ into the direct product of graph towers $\mathfrak{T}_{B}$, where $B$ runs over all the branches of the tree $T_{\text {sol }}(G)$.

We finish this section with the following:

Corollary 8.5 For any finite system of equations $S(X)=1$ over a partially commutative group $\mathbb{G}$, one can find effectively a finite family of graph towers $\mathfrak{T}_{1}, \ldots, \mathfrak{T}_{k}$, $\mathfrak{T}_{i}=\mathbb{G}\left[Y_{i}\right] / S_{i}$ and word mappings $p_{i}: V\left(S_{i}\right) \rightarrow V(S)$ such that for every $b \in V(S)$ there exist $i$ and $c \in V\left(S_{i}\right)$ for which $b=p(c)$, ie

$$
V(S)=p_{1}\left(V\left(S_{1}\right)\right) \cup \cdots \cup p_{k}\left(V\left(S_{k}\right)\right)
$$

and all sets $p_{i}\left(S_{i}\right)$ are irreducible; moreover, every irreducible component of $V(S)$ can be obtained as a closure of some $p_{i}\left(V\left(S_{i}\right)\right)$ in the Zariski topology.

\section{Groups acting on real cubings and limit groups over par- tially commutative groups}

The goal of this section is to give a characterisation of the class of finitely generated fully residually partially commutative groups in terms of their actions on real cubings. In order to obtain such a description, we first need to define a class of spaces where limit groups naturally act, that is, real cubings, as well as the type of action that characterises limit groups over partially commutative groups, that is, essentially free cospecial actions.

As CAT(0) cube complexes (or cubings) are natural higher-dimensional generalisations of simplicial trees, our goal is to define real cubings as higher-dimensional real trees. 
Although ideally one would like to have a geometric or metric description of real cubings, for the purposes of this paper we define them as ultralimits of cubings.

It is clear that if one is to understand the algebraic structure of a group acting on a real cubing, one needs to have a good understanding of the discrete case, namely the structure of groups acting on cubings. Unfortunately, even this case is far from being fully understood and structural results are known only for very specific type of actions, namely free cospecial actions; see Corollary 9.5. Therefore, the lack of a satisfactory (structural) theory of groups acting on cubings imposes a natural restriction on the type of group actions on real cubings one can consider at this point; see Section 10 for further discussion. Having these limitations in mind, we define essentially free cospecial actions on real cubings as actions which are faithful, nontrivial (without global fixed point) and can be approximated by free cospecial actions on cubings (see page 837 for a formal definition).

With these notions in place, we are able to prove the following theorem and corollary.

Theorem Let $G$ be a finitely generated group. The group $G$ acts essentially freely cospecially on a real cubing if and only if it is fully residually partially commutative.

Corollary A finitely generated group $G$ acts freely, essentially freely and cospecially on a real cubing if and only if $G$ is a subgroup of the graph product of free abelian and (nonexceptional) surface groups.

In particular, if the real cubing is a real tree, then $G$ is a (subgroup of) the free product of free abelian groups and (nonexceptional) surface groups.

Furthermore, we naturally carry Sela's definition of limit group over to the context of partially commutative groups (see Definition 9.23) and prove:

Corollary A finitely generated group $G$ is a (geometric) limit group over a partially commutative group if and only if it is fully residually partially commutative.

\subsection{Special cube complexes}

In this section we review the theory of cospecial actions on $\mathrm{CAT}(0)$ cube complexes; see Haglund and Wise [31].

Definition 9.1 A cube complex $X$ is a CW-complex where each $n$-cell $\sigma$ is a standard Euclidean $n$-cube whose attaching map $\varphi_{\sigma}: \partial \sigma \rightarrow X^{(n-1)}$ satisfies the following conditions: 
(1) The restriction of $\varphi_{e}$ to every face of $e$ is a linear homeomorphism onto a cube of one lower dimension.

(2) $\varphi_{\sigma}$ is a homeomorphism onto its image.

We give $X$ the standard CW-topology.

We refer to 1 -cells as edges or as 1 -cubes and to 0 -cells as vertices or 0 -cubes.

Let $X$ denote a cube complex. The link $\operatorname{link}(\sigma)$ of a cube $\sigma$ is a simplicial complex whose $n$-skeleta are defined inductively as follows:

- The set of vertices of $\operatorname{link}(\sigma)$ is $\left\{\tau \in X^{(n+1)} \mid \sigma \in \partial \tau\right\}$.

- The set of $n$-simplices, $n \geq 1$ of $\operatorname{link}(\sigma)$ is

$$
\left\{\left(\tau_{0}, \ldots, \tau_{n}\right) \mid \tau_{i} \in \operatorname{link}(\sigma)^{0} \text { and there is a cube } v \text { such that } \tau_{i} \in \partial v\right\} .
$$

The cube complex $X$ is combinatorially nonpositively curved if each vertex link is flag (that is each complete subgraph is the 1 -skeleton of a simplex). We say that $X$ is combinatorially CAT( 0$)$ whenever $X$ is combinatorially nonpositively curved and simply connected. Following Sageev [51], we call a simply connected combinatorially nonpositively curved cube complex a cubing.

Each cube of $X$ can be given the metric of a standard unit Euclidean cube in $\mathbb{R}^{n}$. One can then put on a cubing $X$ a pseudometric, which, in fact, turns $X$ into a complete CAT(0) metric space $(X, d)$; see Bridson and Haefliger [9].

Lemma 9.2 (Gromov [27]) Let $X$ be a cube complex. Then $X$ is combinatorially nonpositively curved if and only if the length metric $d$ on $X$ is locally CAT(0). In particular, a cube complex is metrically CAT( 0$)$ if and only if it is combinatorially CAT(0).

Hence, the metric and combinatorial geometry of CAT(0) cube complexes are closely connected; see Haglund [29] for more details. We work with nonpositively curved cube complexes from a combinatorial viewpoint and consider them as higher-dimensional analogues of graphs. However, to introduce certain notions, it will be convenient to think of the cube complex as a geometric object.

A midcube in the cube $I_{n}=[0,1] \times \cdots \times[0,1]$ is the subset obtained by restricting one of the coordinates to $\frac{1}{2}$, so the midcube is parallel to two $(n-1)$-faces of $I_{n}$. The edges of $I_{n}$ dual to this midcube are the edges perpendicular to it. The centre of an $n$-cube in a cube complex is the image of $\left(\frac{1}{2}, \ldots, \frac{1}{2}\right)$ from the corresponding $n$-cell. The centre of an edge is its midpoint. 
Given a cube complex $X$, we form a new cube complex $Y_{X}$, whose cubes are the midcubes of cubes of $X$. The vertices of $Y_{X}$ are the midpoints of edges of $X$. The restriction of a $(k+1)$-cell of $X$ to a midcube of $I_{k+1}$ defines the attaching map of a $k$-cell in $Y_{X}$. Each component of $Y_{X}$ is a hyperplane of $X$. An edge of $X$ is dual to some hyperplane $H$ if its midpoint is a vertex of $H$. Each edge $e$ is dual to a unique hyperplane, which we will denote by $H(e)$. Two hyperplanes $A, B$ of a cube complex $X$ intersect if $A \cap B \neq \varnothing$, they cross if they intersect but are not equal.

Set $\mathcal{H}=\mathcal{E} / \|$ and let $[e],[f] \in \mathcal{H}$. Elements of $\mathcal{H}$ are sometimes called (unoriented) combinatorial hyperplanes. Combinatorial hyperplanes are in one-to-one correspondence with hyperplanes. When no confusion arises we refer to combinatorial hyperplanes as simply hyperplanes.

We will use a combinatorial metric defined on the set of vertices of $X$. Let $\mathcal{E}$ be the set of oriented edges of $X$. An edge path or simply a path in $X$ is a finite sequence of oriented edges such that the end of each edge is the origin of its successor. The length of a path is just the number of edges in the sequence. Given two vertices $p, q \in X$ we define the distance dist $(p, q)$ between $p$ and $q$ as the infimum of the lengths of paths between them. One can check that dist is a metric, called the edge-path metric.

Sometimes, we shall consider cube complexes with the rescaled edge-path metric. Let $c \in \mathbb{R}$ and $c>0$, then we define the rescaled edge-path metric $\operatorname{dist}_{c}$ on a cube complex $X$ as follows. For any $p, q \in X$, set

$$
\operatorname{dist}_{c}(p, q)=\frac{\operatorname{dist}(p, q)}{c} \text {. }
$$

As we have already mentioned, essentially, just as graphs, cube complexes are combinatorial objects. Just as any simplicial tree (graph) can be made into a metric tree (graph) by identifying every edge with $[0,1]$, given a cube complex $X$ with a (rescaled) edge-path metric dist ${ }_{c}$, we shall make $X$ into a metric cube complex. We identify every edge of $X$ with $[0, c]$ and endow every cube of $X$ with the $\ell^{1}$ metric. We thereby obtain a metric on $X$ that we denote by $d_{c}$. Observe that for any vertices $p, q \in X$ we have $d_{c}(p, q)=\operatorname{dist}_{c}(p, q)$. Furthermore, any combinatorial isometry of $\left(X, \operatorname{dist}_{c}\right)$ induces an isometry of $\left(X, d_{c}\right)$. We shall consider only combinatorial isometries of cube complexes and for the most part identify $\left(X, d_{c}\right)$ and $\left(X\right.$, dist $\left._{c}\right)$, and abusing the notation and terminology, refer to $\left(X, d_{c}\right)$ as to $\left(X\right.$, dist $\left._{c}\right)$, in particular, in Sections 9.3 and 9.4.

Let $X$ be a cube complex and let $\mathcal{E}$ be the set of edges of $X$. We define the equivalence relation $\|$ as follows: set $e \| f, e, f \in \mathcal{E}$ if and only if there exists a finite sequence of edges $e=e_{1}, \ldots, e_{n}=f$ such that for each $i=1, \ldots, n-1$, the edges $e_{i}$ and $e_{i+1}$ are opposite sides of some $2-$ cube in $X$ oriented in the same direction. 
Special cube complexes were introduced by F Haglund and D Wise [31]. Following Haglund and Wise, we define a special cube complex as a nonpositively curved cube complex which does not have certain pathologies related to its immersed hyperplanes. An immersed hyperplane $D$ crosses itself if it contains two different midcubes from the same cube of $C$. An immersed hyperplane $D$ is $2-$ sided if the map $D \rightarrow C$ extends to a map $D \times I \rightarrow C$ which is a combinatorial map of cube complexes. When $D$ is 2 -sided, it is possible to consistently orient its dual 1-cubes so that any two dual 1 -cubes lying (opposite each other) in the same 2-cube are oriented in the same direction.

An immersed 2-sided hyperplane $D$ selfosculates if for one of the two choices of induced orientations on its dual 1 -cells, some 0 -cube $v$ of $C$ is the initial 0 -cube of two distinct dual 1-cells of $D$. A pair of distinct immersed hyperplanes $D, E$ cross if they contain distinct midcubes of the same cube of $C$. We say $D, E$ osculate, if they have dual 1-cubes which contain a common 0 -cube, but do not lie in a common 2-cube. Finally, a pair of distinct immersed hyperplanes $D, E$ interosculate if they both cross and osculate, meaning that they have dual 1-cubes which share a 0 -cube but do not lie in a common 2-cube. A cube complex is special if all the following hold (see Haglund and Wise [32, Figure 1]):

(1) No immersed hyperplane crosses itself.

(2) Each immersed hyperplane is 2-sided.

(3) No immersed hyperplane selfosculates.

(4) No two immersed hyperplanes interosculate.

All cube complexes we consider are special and connected unless stated otherwise.

Central examples of special cube complexes are cube complexes associated to partially commutative groups. Let $\mathbb{G}$ be a (perhaps infinitely generated) partially commutative group. The 2-complex $X$ of the standard presentation of $\mathbb{G}$ extends to a nonpositively curved cube complex $C(\mathbb{G})$ by adding an $n$-cube (in the form of an $n$-torus) for each set of $n$ pairwise commuting generators. It is well known, see [31, Example 3.3], that:

- Every graph is a special 1-dimensional cube complex.

- Every CAT(0) cube complex is special.

- For any (not necessarily finitely generated) partially commutative group $\mathbb{G}$, the complex $C(\mathbb{G})$ is a special cube complex. 
Given a partially commutative group, throughout this text we denote by $C(\mathbb{G})$ the standard complex of $\mathbb{G}$ and by $\widetilde{C}(\mathbb{G})$ its universal cover. The universal cover $\widetilde{C}(\mathbb{G})$ is a CAT $(0)$ cube complex whose 1 -skeleton is the Cayley graph $\mathrm{Cay}(\mathbb{G})$ of the standard presentation of $\mathbb{G}$.

As the following series of results show, not only $C(\mathbb{G})$ and $\widetilde{C}(\mathbb{G})$ are important examples of special cube complexes, but essentially these examples are universal.

Proposition 9.3 [31, Theorem 4.2] A cube complex $X$ is special if and only if it admits a combinatorial local isometry to the cube complex $C(\mathbb{G})$ of a (not necessarily finitely generated) partially commutative group $\mathbb{G}$. Furthermore, if $X$ is special, then $\pi_{1}(X)$ is a subgroup of $\mathbb{G}$.

As we have already discussed, cubings are a natural generalisation of simplicial trees. While the structure of groups acting (without inversions) on simplicial trees is well understood, the structure of groups acting (essentially) even freely on cubings may be extremely complex, as shown by examples of Burger and Mozes [12]. Proposition 9.3 motivates the definition of a cospecial action. In the case of trees, if a group acts on a tree freely and without inversions (and, therefore, in this case, cospecially), the group is a subgroup of a free group. In the case of cospecial actions on cubings, Proposition 9.3 can be reformulated as follows.

Proposition 9.4 A group $G$ acts freely cospecially on a (not necessarily finite-dimensional) cubing $C$ if and only if it is a subgroup of a (not necessarily finitely generated) partially commutative group.

Furthermore, by construction of the $A$-typing maps (see [31, Sections 3 and 4]) one can extract a more specific result.

Corollary 9.5 Let $G$ be a group acting freely cospecially on a (not necessarily finitedimensional) cubing $C$. Then:

- There exists a combinatorial isometric embedding of $C$ into the universal cover $\widetilde{C}(\mathbb{G})$ of the standard complex of a (not necessarily finitely generated) partially commutative group $\mathbb{G}$.

- The action of $G$ on $C$ extends to a free cospecial action $\alpha$ of $G$ on $\widetilde{C}(\mathbb{G})$.

- The action $\alpha$ is induced by the action of $G$ by left multiplication on the Cayley $\operatorname{graph} \operatorname{Cay}(\mathbb{G})$.

The following result is not hard to deduce from Proposition 9.4. 
Proposition 9.6 Let $G$ be a finitely generated group. Then the group $G$ acts freely cospecially on a finite-dimensional cubing $C$ if and only if it is a subgroup of a finitely generated partially commutative group $\overline{\mathbb{G}}$.

Proof Let $G=\left\langle w_{1}, \ldots, w_{k}\right\rangle$ be a finitely generated subgroup of a (perhaps, infinitely generated) partially commutative group $\mathbb{G}$. Then $G$ is a subgroup of $\overline{\mathbb{G}}=$ $\left\langle\operatorname{alph}\left(w_{1}\right), \ldots, \operatorname{alph}\left(w_{k}\right)\right\rangle$, which in turn is a canonical parabolic subgroup of $\mathbb{G}$. The group $G$ acts (freely) by left multiplication on the Cayley graph Cay $(\overline{\mathbb{G}})$ of $\overline{\mathbb{G}}$. The Cayley graph $\operatorname{Cay}(\overline{\mathbb{G}})$ is the 1 -skeleton of the universal cover of the standard complex $C(\overline{\mathbb{G}})$ of $\overline{\mathbb{G}}$, denoted $\widetilde{C}(\overline{\mathbb{G}})$, and the action of $G$ on Cay $(\overline{\mathbb{G}})$ gives rise to a free cospecial action on $\widetilde{C}(\overline{\mathbb{G}})$.

Conversely, if $G$ is a finitely generated group acting freely and cospecially on a cubing, using an $A$-typing map (see [31, Sections 3 and 4]) one obtains an embedding of $G$ into a (a priori infinitely generated) partially commutative group $\mathbb{G}$. As above, we conclude that as $G$ is finitely generated, so it embeds into a finitely generated canonical parabolic subgroup $\overline{\mathbb{G}}$ of $\mathbb{G}$.

\subsection{Cube complexes of finite width}

Finitely generated partially commutative groups enjoy many nice properties, which are not shared by infinitely generated ones. In our setting, it is crucial that the group $G$ acting cospecially on a cubing $C$ be a subgroup of a finitely generated partially commutative group. We have seen above that if one considers only finitely generated groups, then one can make sure that $G$ is indeed a subgroup of a finitely generated partially commutative group. Alternatively, as is done by Haglund and Wise [31; 32] one can impose the condition that the quotient of $C$ by the action of $G$ have finitely many immersed hyperplanes.

However, both of these approaches have their limitations. On the one hand, requiring that the quotient have only finitely many immersed hyperplanes, imposes strong restrictions not only on the group $G$, but also on the action of $G$ on $C$, for instance, one automatically excludes many noncocompact actions of subgroups of partially commutative groups.

On the other hand, the assumption that the group $G$ is finitely generated is not sufficient in our setting. Suppose we are given a sequence of free actions of finitely generated groups $G_{i}$ on cubings $C_{i}$ (we shall soon see that this is precisely the setting we work with). Each of these actions defines an embedding of $G_{i}$ into a partially commutative group $\mathbb{G}_{i}$. It may happen that the ranks of $\mathbb{G}_{i}$ tend to infinity as $i$ tends to infinity and therefore, we can not find a finitely generated universe for the groups $G_{i}$. In 
Section 10 we formulate a problem for partially commutative groups whose positive solution would resolve this technical issue and allow one to consider arbitrary cubings.

Our next goal is to show that one can impose a natural geometric restriction of finite width on the cubing $C$ which is sufficient to avoid the aforementioned pathologies, namely we are able to show that a group $G$ acts freely cospecially on a cubing of finite width if and only if $G$ is a subgroup of a finitely generated partially commutative group. In fact, it allows us to find a universal partially commutative group that contains all groups $G$ acting freely on cubings of bounded width. Moreover, we show that the free cospecial action of a group $G$ on a cubing of finite width extends to a free cospecial action of $G$ on the universal cover of the standard complex of a finitely generated partially commutative group.

Let $X$ be a special cube complex. Then, by Proposition 9.4, there always exists a partially commutative group $\mathbb{G}$ so that there is a combinatorial local isometry from $X$ to $C(\mathbb{G})$. However, such an isometry is by far nonunique. For example, the $A$-typing map for the Cayley graph of the free group $F_{2}$ of rank 2 results in a free group of countable rank, but, of course, the Cayley graph Cay $\left(F_{2}\right)$ admits a combinatorial local isometry onto $C\left(F_{2}\right)$.

Definition 9.7 Let $[e],[f] \in \mathcal{H}$ be two hyperplanes of some cube complex. Set $[e] \approx[f]$ if and only if every hyperplane $[g] \in \mathcal{H}$ crosses $[e]$ if and only if it crosses $[f]$. One can check that $\approx$ is an equivalence relation. Denote by $\mathfrak{H}=\mathfrak{H}(X)$ the quotient $\mathcal{H} / \approx$.

We say that the cube complex $X$ is $n$-wide or has width $n$ if and only if there exists a combinatorial local isometry from $X$ to $C(\mathbb{E})$, where $\mathbb{E}$ is a partially commutative group such that $|\mathfrak{H}(C(\mathbb{E}))|=n, n \in \mathbb{N}$ and $n$ is minimal with this property, ie for any $\mathbb{E}^{\prime}$ so that $\left|\mathfrak{H}\left(C\left(\mathbb{E}^{\prime}\right)\right)\right|=k<n$, the cube complex $X$ does not admit a combinatorial local isometry onto $C\left(\mathbb{E}^{\prime}\right)$. In this case, we write $w(X)=n$. If $|w(X)|=n$ for some $n \in \mathbb{N}$, we say that $X$ is of finite width, otherwise, we call $X$ infinitely wide.

Throughout this text, given a special cube complex $X$ of width $n$, we denote by $\mathbb{E}(X)$ a partially commutative group so that $w(C(\mathbb{E}(X)))=n$ and there is a combinatorial local isometry from $X$ to $C(\mathbb{E}(X))$.

Remark 9.8 Let $X$ be a special cube complex of width $n$, then there is a combinatorial isometric embedding of the universal cover $\tilde{X}$ into $\widetilde{C}(\mathbb{E})$ for some partially commutative group $\mathbb{E}$, where $w(C(\mathbb{E}))=n$.

Conversely, if $X$ is a cubing which admits a combinatorial isometric embedding into $\widetilde{C}(\mathbb{G})$ so that $w(C(\mathbb{G}))=n$, and $X \rightarrow Y$ is a covering map, then $X, Y$ have finite width and $w(X), w(Y) \leq n$. 
We now record the following observations about the width of cube complexes, which follow directly from the definition.

Lemma 9.9 (1) The width of any graph is one.

(2) The width of the standard cubulation of the euclidean space $E^{n}$ is $n$.

(3) More generally, if $X$ and $Y$ are cube complexes, then $w(X \times Y)=w(X)+$ $w(Y)$.

(4) If the width of $X$ is finite, then $X$ is finite-dimensional.

(5) The width of any compact cube complex is finite. If a cube complex contains only finitely many embedded hyperplanes, then it has finite width.

(6) Let $\mathbb{G}$ be a partially commutative group with the underlying graph $\Gamma$ and $w(C(\mathbb{G}))=N$; then the deflation $\Gamma^{\prime}$ of $\Gamma$ is a finite graph with $N$ vertices and $w(C(\mathbb{G}))=w\left(C\left(\mathbb{G}\left(\Gamma^{\prime}\right)\right)\right.$.

The main result of this section is the following free actions theorem for special cube complexes of finite width. Viewing simplicial trees as 1-dimensional cubings and graphs as 1-dimensional special cube complexes, the next theorem is a natural generalisation of the free actions theorem for trees: a group acts freely without inversions of edges (and hence cospecially) on a tree if and only if it is a subgroup of a finitely generated free group.

Theorem 9.10 Let $G$ be a group. Then $G$ acts freely cospecially on a cubing of width $n$ if and only if $G$ is a subgroup of a partially commutative group $\mathbb{G}$ and $w(C(\mathbb{G}))=n$.

Proof Let $\widetilde{C}(\mathbb{G})$ be the universal cover of $C(\mathbb{G})$ and $w(\widetilde{C}(\mathbb{G}))=n$. Every subgroup $G$ of $\mathbb{G}$ acts (freely) by left multiplication on the Cayley graph Cay( $\mathbb{G})$ of $\mathbb{G}$. Since Cay $(\mathbb{G})$ is the 1 -skeleton of $\widetilde{C}(\mathbb{G})$, we get a free cospecial action of $G$ on $\widetilde{C}(\mathbb{G})$.

Conversely, if $X$ is a cubing of width $n$, then there exists a combinatorial local isometry from $X$ to $C(\mathbb{E})$, where $w(C(\mathbb{E}))=n$. Therefore, by Proposition 9.3, $G$ embeds into $\mathbb{E}$.

Let $\mathbb{G}=\mathbb{G}(\Gamma)$ be a partially commutative group so that $w(C(\mathbb{G}))=n<\infty$. Let $\Gamma^{\prime}$ be the deflation of $\Gamma$. Then, by Lemma $9.9, \Gamma^{\prime}$ has exactly $n$ vertices. Define the graph $\Gamma^{*}$ as follows. The graph $\Gamma^{*}$ is a finite graph with exactly $2 n$ vertices constructed from $\Gamma^{\prime}$. For every vertex $v \in \Gamma^{\prime}$, we introduce two vertices $v_{1}, v_{2} \in \Gamma^{*}$. We set $\left(v_{i}, u_{j}\right) \in E\left(\Gamma^{*}\right)$ if and only if $(v, u) \in E\left(\Gamma^{\prime}\right)$, for all $v, u \in \Gamma^{\prime}, i, j=1,2$. 
Lemma 9.11 In the above notation, if $\mathbb{G}=\mathbb{G}(\Gamma)$ is a partially commutative group so that $w(C(\mathbb{G}))=n<\infty$, then $\mathbb{G}$ is a subgroup of the $2 n$-generated partially commutative group $\mathbb{G}\left(\Gamma^{*}\right)$.

Proof In the above notation, observe that both $\mathbb{G}(\Gamma)$ and $\mathbb{G}\left(\Gamma^{*}\right)$ can be viewed as a graph product of groups with the underlying graph $\Gamma^{\prime}$ and free vertex groups (see Green [26] and Goda [25] for definition and basic properties of graph products). For every vertex $v \in \Gamma^{\prime}$, let $F([v])$ be the free group generated by $\left\{v_{1}^{\prime}, \ldots, v_{k}^{\prime}, \ldots\right\}=[v]$ associated to the corresponding vertex of the graph product $\mathbb{G}(\Gamma)$ and let $F\left(v_{1}, v_{2}\right)$ be the free group generated $\left\{v_{1}, v_{2}\right\}$ associated to the corresponding vertex of the graph product $\mathbb{G}\left(\Gamma^{*}\right)$. Let $\phi_{v}: F([v]) \hookrightarrow F\left(v_{1}, v_{2}\right)$ be an embedding of $F([v])$ into $F\left(v_{1}, v_{2}\right)$.

The embeddings $\phi_{v}, v \in \Gamma^{\prime}$, give rise to an embedding $\phi: \mathbb{G}(\Gamma) \rightarrow \mathbb{G}\left(\Gamma^{*}\right)$. Thus $\mathbb{G}$ is a subgroup of a $2 n$-generated partially commutative group $\mathbb{G}\left(\Gamma^{*}\right)$.

Corollary 9.12 Let $G$ be a group. Then $G$ acts freely cospecially on a cubing of finite width if and only if $G$ is a subgroup of a finitely generated partially commutative group.

Given a special cube complex $X$ of width $n$, it follows from Lemma 9.11 that the corresponding partially commutative group $\mathbb{E}(X)=\mathbb{G}(\Gamma)$ embeds into the $2 n$-generated partially commutative group $\mathbb{G}\left(\Gamma^{*}\right)$. In this particular case, we denote the partially commutative group $\mathbb{G}\left(\Gamma^{*}\right)$ by $\mathbb{P}(X)$ to stress the fact that it is constructed from the complex $X$.

As we have shown above, if a group $G$ acts freely cospecially on a cubing of finite width, then it is a subgroup of a finitely generated partially commutative group $\mathbb{P}$. We now show that there is a universal partially commutative group that contains all groups that act freely cospecially on cubings of a given width.

By Lemma 9.9, for any $N \in \mathbb{N}$ there are only finitely many deflated graphs $\Gamma_{1}, \ldots, \Gamma_{k}$, so that $w\left(C\left(\mathbb{G}\left(\Gamma_{i}\right)\right)\right) \leq N$. Set $\Gamma_{N}$ to be the union of the graphs $\Gamma_{i}^{*}$,

$$
\Gamma_{N}=\Gamma_{1}^{*} \cup \cdots \cup \Gamma_{k}^{*},
$$

and $\mathbb{G}_{N}=\mathbb{G}\left(\Gamma_{N}\right)=\mathbb{G}\left(\Gamma_{1}^{*}\right) * \cdots * \mathbb{G}\left(\Gamma_{k}^{*}\right)$, where the graphs $\Gamma_{i}^{*}$ 's are defined as in the proof of Lemma 9.11. Observe that $\Gamma_{N}$ is a finite graph.

We now arrive at the following:

Corollary 9.13 For every $N \in \mathbb{N}$, there exists a partially commutative group $\mathbb{G}_{N}=$ $\mathbb{G}\left(\Gamma_{N}\right)$ such that every group $G$ acting freely cospecially on a cubing $C$ of width $N$ is a subgroup of $\mathbb{G}_{N}$. 
Our next goal is to show that if a group $G$ acts freely cospecially on a cubing $C$ of width $n$, then one can construct an embedding from $G$ to $\mathbb{P}(C)$ so that it induces an equivariant quasi-isometric embedding from $C$ to the universal cover of $C(\mathbb{P})$. Therefore, the study of groups acting freely cospecially on cubings of finite width reduces to the study of groups acting freely cospecially on the universal covers of standard complexes of partially commutative groups.

Let $G=\langle S\rangle, S=S^{-1}$ be a finitely generated group acting on a cubing $C$, and let $x \in C$. Define the displacement at $x$ to be

$$
\partial_{x}=\max _{s \in S} \operatorname{dist}(s . x, x) .
$$

If the cubing $C$ has a designated based point $b$, then the number $\partial_{b}$ is called the displacement of the action of $G$.

Lemma 9.14 Let $F(A)=F\left(a_{1}, \ldots, a_{r}, \ldots\right), F(B)=F\left(a_{1}, \ldots, a_{r}\right)$ and $F(b, c)$ be free groups on the indicated alphabets. Let $w_{1}, \ldots, w_{r} \in F(b, c)$ be $r$ words in $F(b, c)$ so that $\left|w_{i}\right|=r, w_{i} \neq w_{j}^{ \pm 1}$ and $w_{i}$ does not begin or end with $b^{-1}$ and contains the letter $c, i=1, \ldots, r$. Define the homomorphism $\psi: F\left(a_{1}, \ldots, a_{r}, \ldots,\right) \rightarrow F(b, c)$ as

$$
\psi\left(a_{i}\right)=w_{i} b^{L+i} a_{i} b^{L+i} w_{i}
$$

where $L \in \mathbb{N}, i=1, \ldots, r$ and set

$$
\psi\left(a_{j}\right)=b^{L+r+j} a_{i} b^{L+r+j}
$$

for all $j>r$. Then the map $\psi$ is an embedding of $F(A)$ into $F(b, c)$, the restriction of $\psi$ onto $F(B)$ is a bilipschitz embedding with constant $4 r+2 L+1$.

Proof The proof that $\psi$ is an embedding is standard and left to the reader. Abusing the notation, we denote the restriction of $\psi$ onto $F(B)$ by $\psi$. Since all subgroups of free groups are quasiconvex, we only need to compute the bilipschitz constant.

By definition $2 r+2 L+3 \leq\left|\psi\left(a_{i}\right)\right| \leq 4 r+2 L+1$ and the equalities are attained for $i=1$ and $i=r$, correspondingly. On the other hand, by the definition of $\psi$, it follows that for all $v \in F\left(a_{1}, \ldots, a_{r}\right)$ the length $|\psi(v)|_{F(b, c)}$ satisfies the inequality

$$
2 L|v|_{F\left(a_{1}, \ldots, a_{r}\right)} \leq|\psi(v)|_{F(b, c)} \leq(4 r+2 L+1)|v|_{F\left(a_{1}, \ldots, a_{r}\right)}
$$

and the statement follows. 
Lemma 9.15 Let $F(A)=F\left(a_{1}, \ldots, a_{l}, \ldots\right)$ and $F(b, c)$ be two free groups on the indicated generators. Let $H=\left\langle w_{1}, \ldots, w_{k}\right\rangle<F$ be a finitely generated subgroup of $F(A)$. Reenumerating the generators of $F(A)$, let $B=\left\{a_{1}, \ldots, a_{r}\right\}=$ $\left\{\operatorname{alph}\left(w_{1}\right), \ldots, \operatorname{alph}\left(w_{k}\right)\right\}$. We view $\operatorname{Cay}(F(A))$, Cay $(F(B))$ and $\operatorname{Cay}(F(b, c))$ as 1-dimensional cube complexes based at the identity. The embedding of $H$ into $F(B)$ defines a subcomplex $C(H)$ in $\operatorname{Cay}(F(B))$. We designate the identity as the basepoint of $C(H)$.

Let $\alpha$ be the action of $H$ on $\operatorname{Cay}(F(B))$ by left multiplication, let $\psi$ be the map defined in Lemma 9.14, let $\beta$ be the corresponding action of $H$ on $F(b, c)$ and let $\partial(\alpha)$ and $\partial(\beta)$ be the displacements of the corresponding actions. Then:

- The homomorphism $\psi$ induces an equivariant bilipschitz embedding $\psi^{*}$ of $\operatorname{Cay}(F(B))$ into $\operatorname{Cay}(F(b, c))$, the constant of the embedding $\psi^{*}$ does not exceed $6 k \partial(\alpha)+1$.

- The following inequality holds: $2 k \partial(\alpha)^{2} \leq \partial(\beta) \leq 7 k \partial(\alpha)^{2}$.

- The homomorphism $\psi$ induces an equivariant bilipschitz embedding $\psi^{\prime}$ of $\left(F(B), \operatorname{dist}_{\partial(\alpha)}\right)$ into $\left(\mathrm{Cay}(F(b, c))\right.$, $\left.\operatorname{dist}_{\partial(\beta)}\right)$, the constant of $\psi^{\prime}$ does not exceed $7 k$.

Proof By Lemma 9.14, for $L=k \partial(\alpha)$, the homomorphism $\psi$ induces the bilipschitz embeddings $\psi^{*}$ and $\psi^{\prime}$. We only need to compute the constants.

By definition, the displacement of the action is the displacement of the basepoint of the complex. Therefore, in this case, the displacement $\partial(\alpha)$ equals $\max _{i=1, \ldots, k}\left\{\left|w_{i}\right|_{F(B)}\right\}$. There are at most $k \partial(\alpha)$ different letters in the words $w_{1}, \ldots, w_{k}$, therefore $r \leq k \partial(\alpha)$. By Lemma 9.14, it follows that the constant of $\psi^{*}$ does not exceed $6 k \partial(\alpha)+1$.

The displacement of $\partial(\beta)$ equals $\max _{i=1, \ldots, k}\left\{\left|\psi\left(w_{i}\right)\right|_{F(b, c)}\right\}$. Let $a_{i} \in B$, then $\left|\psi\left(a_{i}\right)\right|=2(r+k \partial(\alpha)+i)+1$. Since $\left|w_{i}\right|_{F(B)} \leq \partial(\alpha)$ and $r \leq k \partial(\alpha)$, we have

$$
\begin{aligned}
\partial(\beta)=\max _{i=1, \ldots, k}\left\{\left|\psi\left(w_{i}\right)\right|_{F(b, c)}\right\} & \leq \max _{i=1, \ldots, k}\left\{\left|w_{i}\right|_{F(B)}\right\} \cdot \max _{j=1, \ldots, k \partial(\alpha)}\left|\psi\left(a_{j}\right)\right|_{F(b, c)} \\
& \leq \max _{i=1, \ldots, k}\left\{\left|w_{i}\right|_{F(B)}\right\} \cdot 7 k \partial(\alpha)=7 k \partial(\alpha)^{2} .
\end{aligned}
$$

On the other hand, since there exists $l, 1 \leq l \leq k$ so that $\left|w_{l}\right|_{F(B)}=\partial(\alpha)$, we have

$$
\partial(\beta) \geq\left|\psi\left(w_{l}\right)\right|_{F(b, c)} \geq(2 k \partial(\alpha)+1) \partial(\alpha) \geq 2 k \partial(\alpha)^{2} .
$$

The last statement now follows from the first two. 
Proposition 9.16 Let $\alpha$ be a free cospecial action of a $k$-generated group $G$ on a based cubing $(C, b)$ of width $n$. Let $\mathbb{E}=\mathbb{E}(C)$ and $\mathbb{P}=\mathbb{P}(C)$ be the corresponding partially commutative groups. Let $\chi: G \hookrightarrow \mathbb{E}(C)$ be the induced embedding and let $\beta^{\prime}$ be the action of $G$ induced by $\alpha$ on $\widetilde{C}(\mathbb{E})$. Let $G=\left\langle w_{1}, \ldots, w_{k}\right\rangle<\mathbb{E}(C)$ and, re-enumerating the generators of $\mathbb{E}(C)$, let $\left\langle\operatorname{alph}\left(w_{1}\right), \ldots, \operatorname{alph}\left(w_{k}\right)\right\rangle=\left\langle a_{1}, \ldots, a_{r}\right\rangle$, where $a_{i}$ are canonical generators of $\mathbb{E}(C), i=1, \ldots, r$.

Let $\psi$ be the embedding of $\mathbb{E}(C)$ into $\mathbb{P}(C)$ defined as in Lemma 9.11 using the maps $\psi_{v}$ from Lemma 9.14, where $L=k \partial(\alpha)$. Denote by $\phi$ the composition of $\chi$ and $\psi$ and let $\beta$ be the action of $G$ induced by $\alpha$ on $\widetilde{C}(\mathbb{P})$. Endow $C, \widetilde{C}(\mathbb{E})$ and $\widetilde{C}(\mathbb{P})$ with the edge-path metric and let the cubings $\widetilde{C}(\mathbb{E})$ and $\widetilde{C}(\mathbb{P})$ be based at lifts of the identity elements id' and id of $\mathbb{E}$ and $\mathbb{P}$, correspondingly. Then:

- The embedding $\phi$ induces an equivariant, based, bilipschitz embedding $\psi^{*}$ of $(C, b)$ into $(\widetilde{C}(\mathbb{P})$, id $)$.

- The following inequality holds $2 k \partial(\alpha)^{2} \leq \partial(\beta) \leq 7 k \partial(\alpha)^{2}$.

- The embedding $\phi$ induces an equivariant, based, $7 k$-bilipschitz embedding from $\left(C, \operatorname{dist}_{\partial(\alpha)}, b\right)$ to $\left(\widetilde{C}(\mathbb{P}), \operatorname{dist}_{\partial(\beta)}\right.$, id $)$.

Proof By Corollary 9.5, the embedding of $G$ into $\mathbb{E}$ induces an equivariant, combinatorial isometric embedding of $C$ into $\widetilde{C}(\mathbb{E})$. Hence, it suffices to show that the embedding $\psi$ of $\mathbb{E}$ into $\mathbb{P}$ and, consequently, the induced map $\psi^{*}$ from $\widetilde{C}(\mathbb{E})$ into $\widetilde{C}(\mathbb{P})$ can be chosen in such a way that the restriction $\left.\psi^{*}\right|_{C}$ of $\psi^{*}$ onto the image of $C$ in $\widetilde{C}(\mathbb{E})$ satisfies the statements of the proposition.

The statement now follows by the definition of $\psi$; see the proof of Lemmas 9.11 and 9.15 .

\subsection{Real cubings}

The aim of this section is to introduce and give examples of the main object of our study, real cubings. We begin by recalling the notion of an ultralimit of metric spaces. We refer the reader to Roe [50] for more details.

Let $\mathfrak{U}$ be a nonprincipal ultrafilter on $\mathbb{N}$. Let $\left(X_{n}, d_{n}\right)$ be a sequence of metric spaces with specified basepoints $p_{n} \in X_{n}$. We say that a sequence $\left(x_{n}\right)_{n \in \mathbb{N}}$, where $x_{n} \in X_{n}$, is admissible if the sequence of real numbers $\left(d_{n}\left(x_{n}, p_{n}\right)\right)_{n \in \mathbb{N}}$ is bounded. Denote the set of all admissible sequences by $\mathfrak{A}$. It is easy to see from the triangle inequality that, for any two admissible sequences $\boldsymbol{x}=\left(x_{n}\right)_{n \in \mathbb{N}}$ and $\boldsymbol{y}=\left(y_{n}\right)_{n \in \mathbb{N}}$, the sequence $\left(d_{n}\left(x_{n}, y_{n}\right)\right)_{n \in \mathbb{N}}$ is bounded and hence there exists a $\mathfrak{U}$-limit $\widehat{d}_{\infty}(\boldsymbol{x}, \boldsymbol{y})=$ $\lim _{\mathfrak{U}} d_{n}\left(x_{n}, y_{n}\right)$. 
Define a relation $\sim$ on the set $\mathfrak{A}$ as follows. Set $\boldsymbol{x} \sim \boldsymbol{y}$ if and only if $\widehat{d}_{\infty}(\boldsymbol{x}, \boldsymbol{y})=0$. It is easy to show that $\sim$ is an equivalence relation on $\mathfrak{A}$.

The ultralimit $\left(X_{\infty}, d_{\infty}\right)=\lim _{\mathfrak{U}}\left(X_{n}, d_{n}, p_{n}\right)$ with respect to $\mathfrak{U}$ of the sequence $\left(X_{n}, d_{n}, p_{n}\right)_{n \in \mathbb{N}}$ is a metric space $\left(X_{\infty}, d_{\infty}\right)$ defined as follows. As a set, we have $X_{\infty}=\mathfrak{A} / \sim$. For two $\sim$ equivalence classes $[\boldsymbol{x}]$ and $[\boldsymbol{y}]$ of admissible sequences $\boldsymbol{x}$ and $\boldsymbol{y}$, we set $d_{\infty}([\boldsymbol{x}],[\boldsymbol{y}])=\widehat{d}_{\infty}(\boldsymbol{x}, \boldsymbol{y})=\lim _{\mathfrak{U}} d_{n}\left(x_{n}, y_{n}\right)$. It is not hard to see that $d_{\infty}$ is well-defined and that it is a metric on the set $X_{\infty}$.

An important class of ultralimits are the so-called asymptotic cones of metric spaces. Let $(X, d)$ be a metric space, let $\mathfrak{U}$ be a nonprincipal ultrafilter on $\mathbb{N}$, let $p_{n} \in X$ be a sequence of basepoints and let $\left\{j_{n}\right\}$ be a sequence of positive integers. Then the $\mathfrak{U}$-ultralimit of the sequence $\left(X, d / j_{n}, p_{n}\right)$ is called the asymptotic cone of $X$ with respect to $\mathfrak{U},\left\{j_{n}\right\}_{n \in \mathbb{N}}$ and $\left\{p_{n}\right\}$ and is denoted $\operatorname{Cone}_{\mathfrak{U}}\left(X_{n}, j_{n}, p_{n}\right)$. The point $\left(p_{n}\right)_{n \in \mathbb{N}}$ is called the observation point and the sequence $\left\{j_{n}\right\}$ is called the scaling sequence. An asymptotic cone of a group $G$ is simply an asymptotic cone of its Cayley graph. Note that it is customary to require that $\lim _{n \rightarrow \infty} j_{n}=\infty$. In our setting, we allow for the possibility that the asymptotic cone of a cubing be a (simplicial) metric cubing.

The following properties of ultralimits of metric spaces are well known; see Kapovich and Leeb [34] and [50].

(1) If $\left(X_{n}, d_{n}\right)_{n \in \mathbb{N}}$ are geodesic metric spaces, then $\lim _{\mathfrak{U}}\left(X_{n}, d_{n}, p_{n}\right)$ is also a geodesic metric space.

(2) The ultralimit $\lim _{\mathfrak{U}}\left(X_{n}, d_{n}, p_{n}\right)$ of metric spaces is a complete metric space.

(3) Let $\kappa \leq 0$ and let $\left(X_{n}, d_{n}\right)_{n \in \mathbb{N}}$ be a sequence of $\operatorname{CAT}(\kappa)$-metric spaces. Then the ultralimit is also a $\operatorname{CAT}(\kappa)$-space.

(4) Let $\left(X_{n}, d_{n}\right)_{n \in \mathbb{N}}$ be a sequence of $\operatorname{CAT}\left(\kappa_{n}\right)$-metric spaces, where $\lim _{\mathfrak{U}} \kappa_{n}=-\infty$. Then $\lim _{\mathfrak{U}}\left(X_{n}, d_{n}, p_{n}\right)$ is a real tree.

We recall that every cubing $X$ can be turned into a metric cubing by endowing every cube of $X$ with the $\ell^{1}$ metric.

Definition 9.17 Let $\left(X_{n}, \text { dist }_{c_{n}}, b_{n}\right)_{n \in \mathbb{N}}$, $\operatorname{dist}_{c_{n}}=\operatorname{dist} / c_{n}$, be a sequence of cubings with fixed based points $b_{n}$ endowed with the metric dist $c_{c_{n}}$, and let $\mathfrak{U}$ be a nonprincipal ultrafilter on $\mathbb{N}$. Suppose that the widths of $X_{i}$ are uniformly bounded by a fixed $N \in \mathbb{N}$. Then we call the ultralimit $\mathcal{C}=\lim _{\mathfrak{U}}\left(X_{n}, \operatorname{dist}_{c_{n}}, b_{n}\right)$ a real cubing.

Let $\omega \in \mathfrak{U}$ and suppose that $w\left(X_{n}\right)=N$, for all $n \in \omega$. Then we say that $\mathcal{C}$ is a real cubing of width $N$. Note that the width of a real cubing is well defined by the properties of the ultrafilters. 
The following proposition follows from well-known results; see $[9 ; 34 ; 50]$.

Proposition 9.18 Let $\mathcal{C}$ be a real cubing, then $\mathcal{C}$ is a complete contractible CAT $(0)$ space.

We now give some examples of real cubings, see Section 10 for further discussion.

Example 9.19 (1) Any asymptotic cone of a finitely generated partially commutative group is a real cubing since it is an ultralimit of the universal cover of the standard complex of the partially commutative group.

(2) It is well known that $\mathrm{SL}_{2}(\mathbb{R})$ is quasi-isometric to the direct product of the hyperbolic plane with the real line, hence every asymptotic cone of $\mathrm{SL}_{2}(\mathbb{R})$ is bilipschitz equivalent to the direct product of a real tree and the real line, and, in fact (see Kar [35]) every asymptotic cone of the universal cover of $\mathrm{SL}_{2}(\mathbb{R})$ endowed with the Sasaki metric is isometric to a real cubing.

(3) The asymptotic cone of any toral relatively hyperbolic group is bilipschitz equivalent to a real cubing; see Osin and Sapir [48] and Sisto [55].

We shall need the following lemma, which is well known and easy to prove.

Lemma 9.20 Let $\left(X_{n}, d_{n}, x_{n}\right)$ and $\left(Y_{n}, d_{n}^{\prime}, y_{n}\right)$ be a sequence of based metric spaces and let $\mathfrak{U}$ be an ultrafilter. Suppose that $\left(X_{n}, d_{n}, x_{n}\right)$ and $\left(Y_{n}, d_{n}^{\prime}, y_{n}\right)$ are $\left(q_{n}, C\right)$ quasi-isometric and $\lim _{\mathfrak{U}} q_{n}=B$. Then $\lim _{\mathfrak{U}}\left(X_{n}, d_{n}, x_{n}\right)$ and $\lim _{\mathfrak{U}}\left(Y_{n}, d_{n}^{\prime}, y_{n}\right)$ are $B$-bilipschitz equivalent.

\subsection{Groups acting on real cubings}

At this point we begin our study of groups acting on real cubings.

It is clear that any (metric) cubing is a real cubing, hence, if one is to understand the structure of groups acting on real cubings, one needs to have a good understanding of group actions on cubings. As we discussed in Sections 2.2 and 9.1, free cospecial group actions on cubings, essentially, are the only actions which are understood. We therefore consider the class of essentially free cospecial actions on real cubings which naturally generalises cospecial actions on (discrete) cubings.

Let $G$ be a group acting cospecially by isometries on a cubing $C$. Let $K=\{g \in G \mid$ $g . x=x$ for all $x \in C\}$ be the kernel of the action. We shall say that the action of $G$ on a cubing is essentially free if $G \neq K$ and the induced action of the group $G / K$ on $C$ is free. 
Definition 9.21 Let $\mathcal{C}=\lim _{\mathfrak{U}}\left(C_{i}, b_{i}\right.$, $\left.\operatorname{dist}_{c_{i}}\right)$ be a real cubing. Let $\left\{\alpha_{i}\right\}$ be a sequence of group actions of a group $G$ on the cubings $\left\{C_{i}\right\}$. Suppose that for all $\left(x_{i}\right) \in \mathcal{C}$ and all $g \in G$ the sequence of elements $\left(g . x_{i}\right)$ is admissible and thus represents an element of $\mathcal{C}$. Define an action $\alpha$ of $G$ on $\mathcal{C}$ by

$$
\alpha\left(g .\left(x_{n}\right)\right)=\left(\alpha_{n}\left(g \cdot x_{n}\right)\right) .
$$

We call such an action limiting and the actions of $G$ on the $C_{i}$ are called the components of the limiting action.

Suppose further that we have a limiting action of $G$ on the real cubing $\mathcal{C}$. We say that $G$ acts essentially freely and cospecially on $\mathcal{C}$ if the action of $G$ on $\mathcal{C}$ is faithful, nontrivial (ie without a global fixed point) and the components of the action are essentially free and cospecial actions.

In [28], Guirardel showed that, in fact, any stable action of a finitely presented group on a real tree can be approximated by actions on simplicial trees. From this perspective, the theory of (stable) actions on real trees is the theory of ultralimits of actions on simplicial trees, $[34 ; 50]$. The main example of essentially free cospecial action on a real tree (viewed as 1-dimensional real cubings) is the action of a limit group $G$ via the (discriminating) family of homomorphisms from $G$ to $F$; see [52].

We now give the central example of essentially free cospecial actions of finitely generated groups on real cubings.

Example 9.22 Let $G$ be a finitely generated group and let $\mathbb{G}$ be a partially commutative group with trivial centre. Let $S=S^{-1}$ be a finite set generating $G$ and let dist be the edge path metric on $\widetilde{C}(\mathbb{G})$. Given an infinite sequence of homomorphisms $\left\{\varphi_{n}: G \rightarrow \mathbb{G}\right\}$, one can associate to it a sequence of positive integers defined by

$$
c_{n}=\max _{x \in \mathbb{G}} \min _{a \in S} \operatorname{dist}\left(\varphi_{n}(a) . x, x\right)=\max _{g \in \mathbb{G}} \min _{a \in S} \operatorname{dist}\left(\mathrm{id}, g^{-1} \varphi_{n}(a) g . \mathrm{id}\right) .
$$

It is well known that if $\left(\varphi_{n}\right)$ are pairwise nonconjugate in $\mathbb{G}$, since $\mathbb{G}$ is finitely generated, then $\lim _{n \rightarrow \infty} c_{n}=\infty$. Since the image $\varphi_{n}(G)$ is a subgroup of $\mathbb{G}$, the subgroup $\varphi_{n}(G)$ acts by left multiplication on the Cayley graph $\operatorname{Cay}(G)$ and thus every homomorphism $\varphi_{n}$ defines an essentially free cospecial action of $G$ on $\widetilde{C}(\mathbb{G})$.

Choose an ultrafilter $\mathfrak{U}$ and let $a \in S, x_{n} \in \mathbb{G}$ be so that $c_{n}=\operatorname{dist}\left(\varphi_{n}(a) . x_{n}, x_{n}\right)$ for $\mathfrak{U}$-almost all $n$. We then obtain a limiting action of $G$ on the asymptotic cone (real cubing) Cone $\mathfrak{U}_{\mathfrak{U}}\left(\mathbb{G} ;\left(x_{n}\right),\left(c_{n}\right)\right)$. By definition, if this action is faithful, then, as we show in Proposition 9.29, it is essentially free and cospecial. 
Following Sela (see [52]) the above example brings us to the definition of a geometric limit group over $\mathbb{G}$.

Definition 9.23 In the notation of Example 9.22, let $L$ be the quotient of $G$ by the kernel $K$ of the action, that is, $K=\left\{g \in G \mid g . x=x\right.$ for all $x \in \operatorname{Cone}_{\mathfrak{U}}\left(\mathbb{G} ;\left(x_{n}\right),\left(c_{n}\right)\right)$. The group $L$ is called a geometric limit group over $\mathbb{G}$.

Note that, by definition, the limit group $L$ over $\mathbb{G}$ acts faithfully on the asymptotic cone of $\mathbb{G}$. However, it is not immediate that this action is essentially free since, a priori, there may not be any nontrivial homomorphisms from $L$ to $\mathbb{G}$.

Given a finitely generated group $G=\langle S\rangle$ acting on a space $X$, it is customary to define the displacement of the action as $\sup _{x \in X} \min _{a \in S} \operatorname{dist}(x, a . x)$. As the following lemma shows, if we are given an essentially free cospecial group action on a real cubing, our definition of displacement and the usual one are basically equivalent. Furthermore, this lemma allows us to change the basepoint of the real cubing.

Lemma 9.24 Let $G=\langle S\rangle$ be a finitely generated group acting essentially freely and cospecially on a real cubing $\mathcal{C}=\lim _{\mathfrak{U}}\left(C_{n}, b_{n} \operatorname{dist}_{c_{n}}\right)$. Then for any $x=\left(x_{n}\right) \in \mathcal{C}$ one has that

$$
\lim _{\mathfrak{U}}\left(\frac{\partial_{b_{n}}}{c_{n}}\right)=p \quad \text { and } \quad \lim _{\mathfrak{U}}\left(\frac{\partial_{b_{n}}}{\partial_{x_{n}}}\right)=q_{x},
$$

where $p, q_{x} \in \mathbb{R}, p, q_{x}>0$.

Proof By definition of the ultralimit, $\lim _{\mathfrak{U}}\left(\left(\partial_{b_{n}}\right) / c_{n}\right)$ always exists. We are to show that $p=\lim _{\mathfrak{U}}\left(\left(\partial_{b_{n}}\right) / c_{n}\right) \neq 0, \infty$. Since the action of $G$ on $\mathcal{C}$ (and, in particular, the basepoint $\left.\left(b_{n}\right)\right)$ is well defined, it follows that $\lim _{\mathfrak{U}}\left(\left(\partial_{b_{n}}\right) / c_{n}\right) \neq \infty$. On the other hand, since the action of $G$ is essentially free and cospecial and therefore nontrivial, we conclude that $p \neq 0$.

The fact that $q_{x} \in \mathbb{R}, q_{x}>0$ follows from the first statement.

Let $G$ be a finitely generated group acting essentially freely and cospecially on a real cubing $\mathcal{C}$ by an action $\alpha=\left\{\alpha_{i}\right\}$ and let the width $w(\mathcal{C})$ of $\mathcal{C}$ be $N$. Then, by Lemma 9.9 and Proposition 9.16, $\mathfrak{U}$-almost all components of the action $\alpha$ define a subgroup $G / K_{i}$ of a partially commutative group $\mathbb{P}_{i}=\mathbb{P}\left(G / K_{i}\right)$, where $w\left(C\left(\mathbb{P}_{i}\right)\right)=N$ and $\mathbb{P}_{i}$ has $2 N$ generators. Since there are only finitely many such partially commutative groups, there exists a partially commutative group $\mathbb{G}(\mathcal{C})$ so that: $\mathbb{G}(\mathcal{C})$ is $2 N$-generated, the width $w(C(\mathbb{G}(\mathcal{C})))$ equals $N$ and $G / K_{i}$ is a subgroup of $\mathbb{G}(\mathcal{C})$ for $\mathfrak{U}$-almost all $i$. Moreover, by Proposition 9.16 , there is a quasi-isometric embedding of $C_{i}$ into $\widetilde{C}(\mathbb{G}(\mathcal{C}))$. Given an essentially free cospecial action of $G$ on $\mathcal{C}$, we call the group $\mathbb{G}(\mathcal{C})=\mathbb{G}(\mathcal{C}, G, \alpha)$ the partially commutative group of $\mathcal{C}$. 
Definition 9.25 Let $\mathbb{G}$ be a partially commutative group and let Cone $\mathfrak{U}(\mathbb{G})$ be its asymptotic cone. An isometry $t$ of Cone $_{\mathfrak{U}}(\mathbb{G})$ is called a (left) translation if there exists an admissible element $w \in \mathbb{G}^{\mathfrak{U}}, w=\left(w_{i}\right)_{i \in \mathbb{N}}$ so that for any point $p=\left(p_{i}\right)_{i \in \mathbb{N}} \in$ $\operatorname{Cone}_{\mathfrak{U}}(\mathbb{G})$ the image $t(p)$ equals $\left(w_{i} p_{i}\right)_{i \in \mathbb{N}}$. The element $w$ is called the vector of the translation $t$. Note that for a translation $t$, the vector $w$ may be defined nonuniquely.

Lemma 9.26 Let $\tau_{r}$ be a translation of $\operatorname{Cone}_{\mathfrak{U}}(\mathbb{G})$ with translation vector $r=$ $\left(r_{i}\right) \in \mathbb{G}^{\mathfrak{U}}$, where $\mathbb{G}$ is a partially commutative group with trivial centre. Then the action $\tau_{r}$ is trivial on all the points of the asymptotic cone Cone $\mathfrak{U}_{\mathfrak{U}}(\mathbb{G})$ if and only if the element $r$ is the trivial element of $\mathbb{G}^{\mathfrak{U}}$.

Proof By definition, the $i^{\text {th }}$ component of $\tau_{r}$ is simply the action on $\widetilde{C}(\mathbb{G})$ defined by the left multiplication by $r_{i}$.

Assume by contradiction that the set $\left\{i \in \mathbb{N} \mid r_{i} \neq 1\right\}$ belongs to the ultrafilter. For every nontrivial $r_{i} \in \mathbb{G}$, we take a canonical generator $y_{i}$ of $\mathbb{G}$ such that $y_{i}$ does not commute with $r_{i}$ and $y_{i}$ does not left-divide $r_{i}$ (see [24] for definition). Notice that such a generator $y_{i}$ exists, since $\mathbb{G}$ has trivial centre.

Let $y=\left(y_{i}^{c_{i}}\right)_{i \in \mathbb{N}}$, where $\left(c_{i}\right)$ is the sequence of the scaling constants, be an element of $\operatorname{Cone}_{\mathfrak{U}}(\mathbb{G})$. Consider the distance $\operatorname{dist}(y, r . y)$. Since the canonical generator $y_{i}$ neither divides nor commutes with $r_{i}$, it follows from [13, Corrollary 4.8] that $y_{i}^{-c_{i}}$ left-divides $y_{i}^{-c_{i}} r_{i} y_{i}^{c_{i}}$, hence $d(y, r . y) \geq 1$ and so the action $\tau_{r}$ is nontrivial on Cone $\mathfrak{U}(\mathbb{G})$.

We now show that the sequence of quasi-isometric embeddings of $C_{i}$ into $\widetilde{C}(\mathbb{G}(\mathcal{C}))$ induces an equivariant bilipschitz embedding of $\mathcal{C}$ into the asymptotic cone of $\mathbb{G}(\mathcal{C})$.

Proposition 9.27 Let $\alpha$ be an essentially free cospecial group action of a $k$-generated group $G$ on a real cubing $\mathcal{C}=\lim _{\mathfrak{U}}\left(C_{n}, b_{n}\right.$, $\left.\operatorname{dist}_{c_{n}}\right)$ and let $\left\{\alpha_{n}\right\}$ be the components of this action. Let $\mathbb{G}(\mathcal{C})$ be the partially commutative group of the real cubing $\mathcal{C}$, let $\beta_{n}$ be the action of $G$ on $\widetilde{C}(\mathbb{G}(\mathcal{C}))$ induced by $\alpha_{n}$ and let $\phi_{n}$ be the embedding of $G / \operatorname{Ker}\left(\alpha_{n}\right)$ into $\mathbb{G}(\mathcal{C})$ constructed in Proposition 9.16. Then there exists a $G-$ equivariant bilipschitz embedding $\psi$ of $\mathcal{C}$ into $\operatorname{Cone}_{\mathfrak{U}}\left(\mathbb{G}(\mathcal{C}), 1, \operatorname{dist}_{\partial\left(\beta_{n}\right)}\right)$.

Proof Let $\psi_{n}$ be the equivariant quasi-isometric embedding of $\left(C_{n}, b_{n}\right.$, dist $\left.{ }_{\partial\left(\alpha_{n}\right)}\right)$ into $\left(\widetilde{C}(\mathbb{G}(\mathcal{C})), 1, \operatorname{dist}_{\partial\left(\beta_{n}\right)}\right)$ constructed in Proposition 9.16. Identifying the 1-skeleton of $\widetilde{C}(\mathbb{G}(\mathcal{C}))$ with the Cayley graph of $\mathbb{G}(\mathcal{C})$, the embedding $\psi_{n}$ maps the basepoint $b_{n}$ to the identity; the basepoint of $\widetilde{C}(\mathbb{G}(\mathcal{C}))$.

By Lemma 9.20, the sequence of maps $\left\{\psi_{n}\right\}$ gives rise to a bilipschitz embedding $\psi$ of $\mathcal{C}$ into Cone $_{\mathfrak{U}}\left(\mathbb{G}(\mathcal{C}), 1, \operatorname{dist}_{\partial\left(\beta_{n}\right)}\right)$ defined by

$$
\psi\left(\left(y_{n}\right)\right)=\left(\psi_{n}\left(y_{n}\right)\right) .
$$


Furthermore, since the actions $\beta_{n}$ are translations, the sequence of actions $\left\{\beta_{n}\right\}$ defines an action $\beta$ of $G$ on $\operatorname{Cone}_{\mathfrak{U}}\left(\mathbb{G}(\mathcal{C}), 1, \operatorname{dist}_{\partial\left(\beta_{n}\right)}\right)$ :

$$
\beta\left(g .\left(x_{n}\right)\right)=\left(\beta_{n}\left(g \cdot x_{n}\right)\right) .
$$

It now follows by Lemma 9.26, that the action $\beta$ is essentially free and cospecial. Since the maps $\psi_{n}$ are equivariant, it follows that $\psi$ is a $G$-equivariant embedding.

The corollary below follows by definition of a translation and by construction of the action of $G$ on Cone $\mathfrak{U}_{\mathfrak{U}}\left(\mathbb{G}_{N}\right)$ given in Proposition 9.27:

Corollary 9.28 Let $G$ be a finitely generated group acting essentially freely and cospecially on a real cubing $\mathcal{C}$. Then $G$ acts essentially freely and cospecially by translations on an asymptotic cone of a finitely generated partially commutative group.

Proof Observe that the action of $G$ on $\operatorname{Cone}_{\mathfrak{U}}\left(\mathbb{G}_{N}, 1, \operatorname{dist}_{\partial\left(\beta_{n}\right)}\right)$ defined in the proof of Proposition 9.27 is by left translations, hence the statement.

We have shown that essentially free cospecial actions on real cubings induce essentially free cospecial actions by left translations on asymptotic cones of partially commutative groups. As we have mentioned above, limit groups over partially commutative groups act essentially freely and cospecially by translations on the corresponding asymptotic cones. We now show that, basically, these are the only examples.

Proposition 9.29 Let $\mathbb{G}$ be a partially commutative group with trivial centre. Let $G$ be a finitely generated group acting faithfully by translations on Cone $\mathfrak{U}(\mathbb{G})$. Then $G$ embeds into the ultrapower $\mathbb{G}^{\mathfrak{U}}$ of $\mathbb{G}$ and so $G$ is fully residually $\mathbb{G}$.

Conversely, let $G$ be a finitely generated fully residually $\mathbb{G}$ group. Then $G$ acts essentially freely and cospecially by translations on the asymptotic cone $\mathrm{Cone}_{\mathfrak{U}}(G)$ of $\mathbb{G}$.

Note that if $G$ acts essentially freely and cospecially by translations, then $G$ acts faithfully and so $G$ satisfies the assumptions of the above proposition.

Proof Let $r=\left(r_{i}\right) \in \mathbb{G}^{\mathfrak{U}}$ be an admissible sequence representing an element of the asymptotic cone Cone $\mathfrak{U}_{\mathfrak{U}}(\mathbb{G})$. Then the element $r$ defines an isometry $\tau_{r}$ of Cone $\mathrm{U}_{\mathfrak{U}}(\mathbb{G})$. The $i^{\text {th }}$ component of $\tau_{r}$ is simply the action on $\widetilde{C}(\mathbb{G})$ defined by left multiplication by $r_{i}$.

Define the map $\psi: G \rightarrow \mathbb{G}^{\mathfrak{U}}$ by sending every generator of $G$ to its translation vector. The map $\psi$ extends to a homomorphism from $G \rightarrow \mathbb{G}^{\mathfrak{U}}$. Indeed, since $G$ acts on 
$\operatorname{Cone}_{\mathfrak{U}}(\mathbb{G})$, it follows that for any relation $r$ of $G$ and any point $y \in \mathrm{Cone}_{\mathfrak{U}}(\mathbb{G})$ we have that the action of $r$ on $y$ is trivial, ie $\operatorname{dist}(y, r . y)=0$. By Lemma 9.26, it then follows that the translation vector $r$ is, in fact, the trivial element of $\mathbb{G}^{\mathfrak{U}}$ and hence $\psi$ is a homomorphism.

The homomorphism $\psi$ gives rise to an action of $G$ on $\mathbb{G}^{\mathfrak{U}}$. Since $G$ acts faithfully on Cone $_{\mathfrak{U}}(\mathbb{G})$, the induced action of $G$ on $\mathbb{G}^{\mathfrak{U}}$ is faithful. Therefore, the homomorphism $\psi$ is injective. We conclude that $G$ is fully residually $\mathbb{G}$ since so is every subgroup of $\mathbb{G}^{\mathfrak{U}}$; see Theorem 2.5.

On the other hand, any fully residually $\mathbb{G}$ group $G$ can be viewed as a subgroup of $\mathbb{G}^{\mathfrak{U}}$. Therefore, $G$ acts faithfully on $\mathbb{G}^{\mathfrak{U}}$ by left translations. Furthermore, since the group $G$ is finitely generated one can choose scaling constants so that every translation vector corresponding to an element of $G$ is admissible in the asymptotic cone and hence the group $G$ acts on $\operatorname{Cone}_{\mathfrak{U}}(\mathbb{G})$. Since the action of $G$ on $\mathbb{G}^{\mathfrak{U}}$ is faithful, the translation vector $\left(g_{i}\right)$ corresponding to a nontrivial element $g$ of $G$ is nontrivial. By Lemma 9.26, the action of $\left(g_{i}\right)$ on the asymptotic cone is nontrivial. It follows that the action of $G$ on $\operatorname{Cone}_{\mathfrak{U}}(\mathbb{G})$ is faithful.

Let $\mathbb{G}$ be the free abelian group of finite rank $n$ and let Cone $\mathfrak{U}_{\mathfrak{U}}(\mathbb{G})$ be its asymptotic cone. Then $\operatorname{Cone}_{\mathfrak{U}}(\mathbb{G})$ is isometric to $\mathbb{R}^{n}$ with the $\ell^{1}$ metric. It is not hard to see that if a finitely generated group $G$ acts faithfully by translations on $\mathbb{R}^{n}$, then $G$ is free abelian.

Corollary 9.30 Let $\mathbb{G}$ be an arbitrary partially commutative group and $Z(\mathbb{G})$ be its centre, ie $\mathbb{G}=\mathbb{G}^{\prime} \times Z(\mathbb{G})$ and $Z(\mathbb{G})=\mathbb{Z}^{n}$. Let $G$ be a finitely generated group acting faithfully by translations on Cone $\operatorname{CU}_{\mathfrak{U}}(\mathbb{G})=$ Cone $_{\mathfrak{U}}\left(\mathbb{G}^{\prime}\right) \times \mathbb{R}^{n}$, then $G$ is a subgroup of a group of the form $G^{\prime} \times \mathbb{Z}^{m}$, where $G^{\prime}$ is a finitely generated group acting faithfully by translations on Cone $\mathfrak{U}\left(\mathbb{G}^{\prime}\right)$. Hence, $G \simeq G^{\prime \prime} \times \mathbb{Z}^{l}$, where $G^{\prime \prime}$ is a finitely generated group acting faithfully by translations on Cone $\mathfrak{U}\left(\mathbb{G}^{\prime}\right)$. Moreover, $G$ is fully residually $\mathbb{G}$.

Proof Let $G=\left\langle x_{1}, \ldots, x_{k}\right\rangle$ be a set of generators of $G$ and let $v^{i}$ be the translation vector of $x_{i}, i=1, \ldots, k$. Write $v^{i}=\left(\left(u_{n}^{i}, w_{n}^{i}\right)\right)_{n \in \mathbb{N}}$, where $u_{n}^{i} \in \mathbb{G}^{\prime}$ and $w_{n}^{i} \in \mathbb{Z}^{n}$ for all $i$ and $n$. Consider the subgroup $H$ of isometries of Cone $\mathfrak{U}(\mathbb{G})$ generated by the $2 k$ translations $\left\{\left(u_{n}^{1}\right), \ldots,\left(u_{n}^{k}\right), \ldots,\left(w_{n}^{1}\right), \ldots,\left(w_{n}^{k}\right)\right\}$. It is clear that $H=$ $\left\langle\left(u_{n}^{1}\right), \ldots,\left(u_{n}^{k}\right)\right\rangle \times\left\langle\left(w_{n}^{1}\right), \ldots,\left(w_{n}^{k}\right)\right\rangle$ and that $G$ is a subgroup of $H$. Furthermore, since if $u_{n}^{i} \neq 1$ for almost all $n$, by Lemma 9.26, there exists an element of the asymptotic cone Cone $_{\mathfrak{U}}\left(\mathbb{G}^{\prime}\right)$ which is not fixed by the action by $\left(u_{n}^{i}\right)$. Therefore $G^{\prime}=\left\langle\left(u_{n}^{1}\right), \ldots,\left(u_{n}^{k}\right)\right\rangle$ acts faithfully by translations on Cone $\mathfrak{U}\left(\mathbb{G}^{\prime}\right)$. Finally, the group $\left\langle\left(w_{n}^{1}\right), \ldots,\left(w_{n}^{k}\right)\right\rangle$ acts by translations on $\mathbb{R}^{n}$ and thus is free abelian. 
In the case of free groups, Sela introduced the notion of geometric limit group as the quotient of a finitely generated group by the kernel of the action induced by a family of homomorphisms on a real tree and proved that the class of geometric limit groups is precisely the class of finitely generated fully residually free groups; see [52].

The above corollary gives a characterisation of the class of geometric limit groups over partially commutative groups as the class of finitely generated fully residually partially commutative groups.

Corollary 9.31 Geometric limit groups over partially commutative groups are precisely finitely generated fully residually partially commutative groups.

Proof It is easy to see that finitely generated fully residually partially commutative groups are geometric limit groups; see Example 9.22.

Assume now that we have an infinite family of (nonpairwise conjugate) homomorphisms $\varphi_{n}: G \rightarrow \mathbb{G}$. Then the group $G$ has a natural action (perhaps unfaithful) by translations on the asymptotic cone Cone $(\mathbb{U})$ of $\mathbb{G}$. Let $L$ be a geometric limit group over $\mathbb{G}$ defined as the quotient of $G$ by the kernel of the action, ie $L$ is the quotient of $G$ by the normal subgroup generated by elements $g \in G$ that have trivial action, ie $g x=x$ for all $x \in$ Cone $_{\mathfrak{U}}(\mathbb{G})$. By definition, the geometric limit group $L$ acts faithfully by translations on the asymptotic cone of $\mathbb{G}$, hence, by Corollary $9.30, L$ is fully residually $\mathbb{G}$.

We summarise the results of this section in the following theorem.

Theorem 9.32 Let $G$ be a finitely generated group. Then:

(1) The group $G$ acts essentially freely and cospecially on a real cubing if and only if $G$ acts essentially freely and cospecially on an asymptotic cone of a partially commutative group $\mathbb{G}$ if and only if $G$ is fully residually $\mathbb{G}$ for some finitely generated partially commutative group.

(2) The group $G$ acts essentially freely and cospecially on a real cubing of width $n$ if and only if $G$ acts essentially freely and cospecially on an asymptotic cone of an $n$-wide and $2 n$-generated partially commutative group $\mathbb{G}$ if and only if $G$ is fully residually $\mathbb{G}$.

Theorem 9.33 Let $G$ be a finitely generated group. The group $G$ acts essentially freely cospecially on a real cubing if and only if it is a subgroup of a graph tower.

Proof The proof follows from Theorems 9.32 and 8.1. 
In the case of free actions, the above theorem results in the following corollary, which can be likened to Rips' theorem on free actions on real trees.

Corollary 9.34 A finitely generated group $G$ acts freely, essentially freely and cospecially on a real cubing if and only if $G$ is a subgroup of the graph product of free abelian and (nonexceptional) surface groups.

In particular, if the real cubing is a real tree, then $G$ is a (subgroup of) the free product of free abelian groups and (nonexceptional) surface groups.

\section{Open problems and future directions}

\subsection{Partially commutative groups}

The notion of tribes has played a key role in our analysis of the of coordinate groups over partially commutative groups. In particular, in order to obtain a finite tribal solution tree encoding all homomorphisms, it is essential that the lattice of tribes be finite. To assure that this condition holds, we define real cubings as ultralimits of cubings of bounded width. Although being a sufficient condition, it is by no means a necessary one. An elegant way to overcome this technical obstacle would be giving by a positive answer to the following question.

Question 1 Given $k \in \mathbb{N}$, does there exist a universal finitely generated partially commutative group $\mathbb{G}_{k}$ that contains all $k$-generated subgroups of arbitrary partially commutative groups?

Or, in a stronger form, given $k \in \mathbb{N}$, does there exist a finitely generated partially commutative group $\mathbb{G}_{k}$ so that for any $k$-generated subgroup $H$ of any partially commutative group $\mathbb{G}$ there exists a homomorphism $\varphi_{H}$ from $\mathbb{G}$ to $\mathbb{G}_{k}$ which is injective on $H$ ?

We note that by Baudisch [2], every 2-generated subgroup of any partially commutative group is either free or free abelian. Hence, there does exist a 2-universal partially commutative group.

\subsection{Real cubings}

Real trees were introduced independently by Chiswell [16] and Tits [57], and, originally, had the requirement that the space should be complete. Later, Alperin and Moss [1] gave a more general definition of real trees as spaces where every two points can 
be joined by a unique arc or, equivalently, as 0 -hyperbolic spaces; hence nowadays completeness of real trees is assumed only when necessary.

Similarly, our definition of real cubings via ultralimits implies that real cubings are complete metric spaces. In order to pass to a more general class of spaces, one can take the following approach. As real trees can be described as metric spaces that are isometric to (convex) subspaces of the asymptotic cone of a free group (see Mayer, Nikiel and Oversteegen [45] and Dyubina and Polterovich [23]) we suggest to define real cubings as (convex) subspaces of the asymptotic cone of a finitely generated partially commutative group (with the metric induced by the $\ell_{1}$-metric on the cubings). The following problem now arises naturally.

Problem 1 Give a metric/geometric description of real cubings.

Once a geometric description of real cubings is achieved, it is natural to ask which of the topological properties of real trees carry over to this setting. As an example, one can ask if real cubings are injective metric spaces.

\subsection{Limiting actions}

An important connection between Bass-Serre theory and Rips' theory was established by Guirardel in [28]. Essentially, he proves that the theory of stable actions on real trees is the theory of ultralimits of Bass-Serre actions on simplicial trees. As we already stressed, if one seeks to establish robust structural results for groups acting on real cubings, one must impose constraints on the type of actions under consideration.

In this paper we assumed that the action on the real cubing is essentially free and cospecial, that is, a particular type of limit of discrete actions. This raises two natural questions: what type of actions on real trees are covered by essentially free cospecial actions and what type of actions on real cubings are approximated by simplicial ones?

More precisely, in the case of real trees (viewed as real cubings), one can show that every very small action of a free group, every faithful, nontrivial action of an abelian group and every small action of the fundamental group of an orientable surface is of our type. If the following question had a positive answer, then we would be able to show that any free (and every very small) action of a group on a real tree is in fact essentially free cospecial.

Question 2 (cf discussion after Corollary 1 in [28]) Can every very small action of the fundamental group of a nonorientable surface on real trees be approximated by very small simplicial actions? 
In the setting of real cubings Guirardel's work naturally raises the following:

Question 3 Is there a nice (geometric) description of actions on real cubings that can be approximated by cospecial actions on cubings?

\subsection{Actions of cubings}

From this perspective, in order to analyse more general actions on real cubings (at least those that are limits of discrete actions) one is to understand the discrete case, that is, to develop a generalisation of the Bass-Serre theory for groups acting (nonfreely) cospecially on cubings.

Problem 2 Develop a higher-dimensional analogue of the Bass-Serre theory, ie establish the structure of groups acting cospecially on cubings.

To clarify the statement we seek, let us mention that in the case when the stabilisers of maximal (by inclusion) cubes are trivial, we expect to obtain the following type of result. If a group $G$ acts cospecially on a cubing with trivial stabilisers of maximal cubes, then $G$ is a subgroup of a graph product of groups. Conversely, if $G$ is a subgroup of a graph product of groups whose vertex groups have nontrivial cospecial actions on cubings, then so does $G$.

\subsection{Actions on real cubings}

Trees have very simple geometry which translates to a very robust algebraic structure of finitely generated groups acting (stably, minimally and nontrivially) on them. The theory of groups acting on real trees has proven to be very rich and finding a higherdimensional generalisation of this theory is a very interesting open problem. Indeed, in recent years, several attempts at finding such generalisation have been made, such as median spaces and spaces with measured walls. The class of real cubings we introduced is yet another such generalisation. Our expectation however is that by imposing some natural constraints on the type of group action under consideration, one should be able to develop a robust theory of groups acting on real cubings that fully and naturally generalises the theory of groups acting on real trees.

As we discussed in the introduction, there is a very close relation between Rips' machine to analyse the dynamics of an action of a finitely generated group on a real tree and the Makanin-Razborov process which describes homomorphisms from finitely generated groups to free groups. Our hope is that good actions on real cubings can be encoded into multifoliated band complexes, which can be interpreted as constrained generalised equations and that the procedure we describe in this paper will guide the analysis of the corresponding dynamics. 
Question 4 The dynamics of which class of actions of finitely generated groups on real cubings can be encoded into constrained generalised equations? What is the structure of such groups?

\subsection{Coarse median algebras}

There is a natural correspondence between median spaces and cubings, namely, any cubing is a discrete median space and vice-versa. In [8], Bowditch introduced the notions of a coarse median spaces and groups and showed, among other things, that hyperbolic groups are coarse median groups. We expect that graph towers are coarse median groups.

Problem 3 Prove that graph towers are coarse median groups.

However, if one drops the condition of discreteness, there is no such a direct geometrical interpretation of median algebras; see [8]. It is clear that real cubings can be given a natural structure of median spaces but the converse is not be true (for instance, median spaces can be infinite-dimensional). Hence, a natural question to ask is under which conditions does the converse hold, for instance:

Question 5 Is there an analogue of the concept of finite width for median spaces which implies (or characterises) that a median space is a real cubing?

In particular, this approach can be helpful to further understand asymptotic cones of coarse median groups such as, prominently, asymptotic cones of mapping class groups.

Indeed, in [8], Bowditch proves that mapping class groups are coarsely median and so their asymptotic cones are median spaces. We hope that, in fact, asymptotic cones of mapping class groups are real cubings:

Problem 4 Prove that asymptotic cones of mapping class groups are (bilipschitz equivalent to) a real cubing.

\subsection{Graph towers}

Once a new class of groups is introduced and especially when this class has such a robust algebraic structure as graph towers, one can ask if one's favourite property holds for this class of groups. We point out some of the properties we expect graph towers to have.

One of the consequences of Wise's work on groups with quasiconvex hierarchies is that limit groups over free groups are virtually special. It is natural to expect that graph towers (and so all limit groups over partially commutative groups) are virtually special. 
Problem 5 Prove that graph towers are virtually special.

Since any tower (and any limit group) is residually partially commutative, the word problem for graph towers (and limit groups) is solvable. Naturally, the next decidability question to ask is the decidability of the conjugacy problem in this class.

Problem 6 Prove that the conjugacy problem for graph towers is decidable.

More generally, in [46] Minasyan proved that partially commutative groups are hereditary conjugacy separable. Minasyan's work leads us to believe that so are graph towers.

Problem 7 Prove graph towers are hereditary conjugacy separable.

In [30], Haglund showed that quasiconvex subgroups of partially commutative groups are separable. It seems to us that this result can be generalised to graph towers:

Problem 8 Prove quasiconvex subgroups of graph towers are separable and virtual retracts.

In a different direction, the rank gradient and homology has been recently computed for both partially commutative groups and limit groups over free groups; see Kar and Nikolov [36] and Bridson and Kochloukova [11]. This raises an analogous problem for graph towers.

Problem 9 Compute the rank gradient and homology of a graph tower.

Finally, one can ask if graph towers play a similar role in the model theory of partially commutative groups as do $\omega$-residually free towers in the model theory of free groups. The following question was suggested by the referee:

Question 6 Does the implicit function theorem hold for graph towers over $\mathbb{G}$, cf Kharlampovich and Myasnikov [40]? Equivalently, do there exist formal solutions in covering closures of graph towers for $\forall \exists$-formulas in $\mathbb{G}$, comparable with Sela [53]?

Answering this question would be the fist step towards understanding finitely generated models of the $\forall \exists$-theory of $\mathbb{G}$. 


\subsection{Limit group over partially commutative groups}

We would like to note that most of the problems given above (in Section 10.7) are hopeless for limit groups over partially commutative groups, since, for instance, all residually free groups are limit groups over some partially commutative group. In particular, the conjugacy problem, the isomorphism problem, etc are undecidable for the class of limit groups over partially commutative groups. Furthermore, while graph towers are CAT(0)-groups (and so many of the techniques of CAT(0)-geometry can be used to study them), limit groups over partially commutative groups in general are not CAT(0).

However, there is a number of very interesting problems for limit groups over partially commutative groups, among them we only mention one. In his work [44], Louder proved that limit groups (over free groups) have finite Krull dimension. As limit groups over partially commutative groups can be extremely complicated, it is not clear if partially commutative groups have finite Krull dimension and we would like to ask:

Question 7 Do limit groups over partially commutative groups have finite Krull dimension?

Note that one of key tool's of Louder's proof is the abelian JSJ-decomposition, which brings us to our last question.

\subsection{JSJ-decomposition}

The abelian JSJ-decomposition played a key role in Sela's approach to limit groups and, in fact, to Tarski problems. Recently, Guirardel and Levitt developed a general theory of JSJ-decompositions for finitely presented groups over arbitrary classes of groups. Furthermore, with additional hypothesis on either the class of groups in question (say, abelian), or on the type of actions (say, acylindrical), one can extend the JSJ-theory to all finitely generated groups. In this context, the results of this paper, in particular the algebraic description of graph towers, seem to indicate that the splittings that arise in the hierarchical construction of limit groups over partially commutative groups correspond to the JSJ over the class of partially commutative groups (and their subgroups). Since limit groups over partially commutative groups are not necessarily finitely presented, one can ask if the JSJ-decomposition over partially commutative groups (and their subgroups) exists for all finitely generated groups. Since a positive answer to this question seems unlikely, following Sela's ideas, one can ask if there is a condition on the type of action (generalising acylindricity) which assures the existence of such a JSJ-decomposition for finitely generated groups and which includes the actions of limit groups over partially commutative groups. 


\section{References}

[1] R C Alperin, K N Moss, Complete trees for groups with a real-valued length function, J. London Math. Soc. 31 (1985) 55-68 MR810562

[2] A Baudisch, Kommutationsgleichungen in semifreien Gruppen, Acta Math. Acad. Sci. Hungar. 29 (1977) 235-249 MR0463300

[3] G Baumslag, A Myasnikov, V N Remeslennikov, Algebraic geometry over groups, I: Algebraic sets and ideal theory, J. Algebra 219 (1999) 16-79 MR1707663

[4] J Behrstock, R Charney, Divergence and quasimorphisms of right-angled Artin groups, Math. Ann. 352 (2012) 339-356 MR2874959

[5] M Bestvina, N Brady, Morse theory and finiteness properties of groups, Invent. Math. 129 (1997) 445-470 MR1465330

[6] R Bieri, Normal subgroups in duality groups and in groups of cohomological dimension 2, J. Pure Appl. Algebra 7 (1976) 35-51 MR0390078

[7] V Blatherwick, Centralisers and big powers in partially commutative groups, $\mathrm{PhD}$ thesis, University of Newcastle-upon-Tyne (2007)

[8] B H Bowditch, Coarse median spaces and groups, Pacific J. Math. 261 (2013) 53-93 MR3037559

[9] M R Bridson, A Haefliger, Metric spaces of nonpositive curvature, Grundl. Math. Wissen. 319, Springer (1999) MR1744486

[10] M R Bridson, J Howie, C F Miller III, H Short, Finitely presented residually free groups arXiv:0809.3704

[11] M R Bridson, DH Kochloukova, Volume gradients and homology in towers of residually-free groups arXiv:1309.1877

[12] M Burger, S Mozes, Finitely presented simple groups and products of trees, C. R. Acad. Sci. Paris Sér. I Math. 324 (1997) 747-752 MR1446574

[13] M Casals-Ruiz, I V Kazachkov, Elements of algebraic geometry and the positive theory of partially commutative groups, Canad. J. Math. 62 (2010) 481-519 MR2666386

[14] M Casals-Ruiz, I V Kazachkov, On systems of equations over free partially commutative groups, Mem. Amer. Math. Soc. 212, Amer. Math. Soc. (2011) MR2817144

[15] C Champetier, V Guirardel, Limit groups as limits of free groups, Israel J. Math. 146 (2005) 1-75 MR2151593

[16] I M Chiswell, Abstract length functions in groups, Math. Proc. Cambridge Philos. Soc. 80 (1976) 451-463 MR0427480

[17] L P Comerford, Jr, C C Edmunds, Quadratic equations over free groups and free products, J. Algebra 68 (1981) 276-297 MR608536 
[18] J Crisp, B Wiest, Embeddings of graph braid and surface groups in right-angled Artin groups and braid groups, Algebr. Geom. Topol. 4 (2004) 439-472 MR2077673

[19] E Daniyarova, A Myasnikov, V N Remeslennikov, Unification theorems in algebraic geometry, from: "Aspects of infinite groups", (B Fine, G Rosenberger, D Spellman, editors), Algebra Discrete Math. 1, World Scientific, Singapore (2008) 80-111 MR2571513

[20] V Diekert, A Muscholl, Solvability of equations in graph groups is decidable, Internat. J. Algebra Comput. 16 (2006) 1047-1069 MR2286422

[21] G Duchamp, D Krob, Partially commutative Magnus transformations, Internat. J. Algebra Comput. 3 (1993) 15-41 MR1214003

[22] A J Duncan, I V Kazachkov, V N Remeslennikov, Parabolic and quasiparabolic subgroups of free partially commutative groups, J. Algebra 318 (2007) 918-932 MR2371978

[23] A Dyubina, I Polterovich, Explicit constructions of universal $\mathbb{R}$-trees and asymptotic geometry of hyperbolic spaces, Bull. London Math. Soc. 33 (2001) 727-734 MR1853785

[24] E S Esyp, I V Kazachkov, V N Remeslennikov, Divisibility theory and complexity of algorithms for free partially commutative groups, from: "Groups, languages, algorithms”, (A V Borovik, editor), Contemp. Math. 378, Amer. Math. Soc. (2005) 319-348 MR2159318

[25] KM Goda, Centralisers in graph products of groups, $\mathrm{PhD}$ thesis, University of Newcastle-upon-Tyne (2006)

[26] E R Green, Graph products of groups, $\mathrm{PhD}$ thesis, University of Leeds (1990)

[27] M Gromov, Hyperbolic groups, from: "Essays in group theory", (S M Gersten, editor), Math. Sci. Res. Inst. Publ. 8, Springer, New York (1987) 75-263 MR919829

[28] V Guirardel, Approximations of stable actions on $\mathbb{R}$-trees, Comment. Math. Helv. 73 (1998) 89-121 MR1610591

[29] F Haglund, Isometries of CAT(0) cube complexes are semisimple arXiv:0705.3386

[30] F Haglund, Finite index subgroups of graph products, Geom. Dedicata 135 (2008) 167-209 MR2413337

[31] F Haglund, D T Wise, Special cube complexes, Geom. Funct. Anal. 17 (2008) 15511620 MR2377497

[32] F Haglund, D T Wise, Coxeter groups are virtually special, Adv. Math. 224 (2010) 1890-1903 MR2646113

[33] S P Humphries, On representations of Artin groups and the Tits conjecture, J. Algebra 169 (1994) 847-862 MR1302120 
[34] M Kapovich, B Leeb, On asymptotic cones and quasi-isometry classes of fundamental groups of 3-manifolds, Geom. Funct. Anal. 5 (1995) 582-603 MR1339818

[35] A Kar, Asymptotically CAT(0) groups, Publ. Mat. 55 (2011) 67-91 MR2779576

[36] A Kar, N Nikolov, Rank gradient and cost of Artin groups and their relatives arXiv: 1210.2873

[37] O Kharlampovich, A Myasnikov, Irreducible affine varieties over a free group, I: Irreducibility of quadratic equations and Nullstellensatz, J. Algebra 200 (1998) 472516 MR1610660

[38] O Kharlampovich, A Myasnikov, Irreducible affine varieties over a free group, II: Systems in triangular quasiquadratic form and description of residually free groups, $\mathbf{J}$. Algebra 200 (1998) 517-570 MR1610664

[39] O Kharlampovich, A Myasnikov, Effective JSJ-decompositions, from: “Groups, languages, algorithms", (A V Borovik, editor), Contemp. Math. 378, Amer. Math. Soc. (2005) 87-212 MR2159316

[40] O Kharlampovich, A Myasnikov, Implicit function theorem over free groups, J. Algebra 290 (2005) 1-203 MR2154989

[41] O Kharlampovich, A Myasnikov, Elementary theory of free nonabelian groups, J. Algebra 302 (2006) 451-552 MR2293770

[42] M R Laurence, A generating set for the automorphism group of a graph group, J. London Math. Soc. 52 (1995) 318-334 MR1356145

[43] Y Liu, Virtual cubulation of nonpositively curved graph manifolds, J. Topol. 6 (2013) 793-822 MR3145140

[44] L Louder, Krull dimension for limit groups, Geom. Topol. 16 (2012) 219-299 MR2872582

[45] J C Mayer, J Nikiel, L G Oversteegen, Universal spaces for $\mathbb{R}$-trees, Trans. Amer. Math. Soc. 334 (1992) 411-432 MR1081940

[46] A Minasyan, Hereditary conjugacy separability of right-angled Artin groups and its applications, Groups Geom. Dyn. 6 (2012) 335-388 MR2914863

[47] A Myasnikov, V N Remeslennikov, Algebraic geometry over groups, II: Logical foundations, J. Algebra 234 (2000) 225-276 MR1799485

[48] D Osin, M Sapir, Universal tree-graded spaces and asymptotic cones, Internat. J. Algebra Comput. 21 (2011) 793-824 MR2827204

[49] V N Remeslennikov, $\exists$-free groups, Siberian Math. J. 30 (1989) 193-197 MR1043446

[50] J Roe, Lectures on coarse geometry, Univ. Lecture Series 31, Amer. Math. Soc. (2003) MR2007488 
[51] M Sageev, Ends of group pairs and nonpositively curved cube complexes, Proc. London Math. Soc. 71 (1995) 585-617 MR1347406

[52] Z Sela, Diophantine geometry over groups, I: Makanin-Razborov diagrams, Publ. Math. Inst. Hautes Études Sci. (2001) 31-105 MR1863735

[53] Z Sela, Diophantine geometry over groups, II: Completions, closures and formal solutions, Israel J. Math. 134 (2003) 173-254 MR1972179

[54] Z Sela, Diophantine geometry over groups, VI: The elementary theory of a free group, Geom. Funct. Anal. 16 (2006) 707-730 MR2238945

[55] A Sisto, Bilipschitz types of tree-graded spaces arXiv:1010.4552

[56] J Stallings, A finitely presented group whose 3-dimensional integral homology is not finitely generated, Amer. J. Math. 85 (1963) 541-543 MR0158917

[57] J Tits, A "theorem of Lie-Kolchin" for trees, from: "Contributions to algebra (collection of papers dedicated to Ellis Kolchin)”, (H Bass, P J Cassidy, J Kovacic, editors), Academic Press, New York (1977) 377-388 MR0578488

[58] H Wilton, Solutions to Bestvina \& Feighn's exercises on limit groups, from: "Geometric and cohomological methods in group theory", (M R Bridson, P H Kropholler, I J Leary, editors), London Math. Soc. Lecture Note Ser. 358, Cambridge Univ. Press (2009) 30-62 MR2605175

M C-R: Mathematical Institute, University of Oxford

Radcliffe Observatory Quarter, Woodstock Road, Oxford OX2 6GG, UK

I K: Department of Mathematics, University of the Basque Country UPV/EHU

Barrio Sarriena, 48940 Leioa, Vizcaya, Spain

IKERBASQUE, Basque Foundation for Science

Bilbao, Spain

montsecasals@gmail.com, ilya.kazachkov@gmail.com

Proposed: Walter Neumann

Received: 2 May 2012

Seconded: Mikhail Gromov, Yasha Eliashberg

Revised: 5 April 2014 